University of Louisville

ThinkIR: The University of Louisville's Institutional Repository

Electronic Theses and Dissertations

$12-2020$

\title{
Development and applications of condensed phase cavity ring- down spectroscopy for studies of electrochemical and interfacial.
}

Shadi Ahmad Alnaanah

University of Louisville

Follow this and additional works at: https://ir.library.louisville.edu/etd

Part of the Atomic, Molecular and Optical Physics Commons

\section{Recommended Citation}

Alnaanah, Shadi Ahmad, "Development and applications of condensed phase cavity ring-down spectroscopy for studies of electrochemical and interfacial." (2020). Electronic Theses and Dissertations. Paper 3555.

https://doi.org/10.18297/etd/3555

This Doctoral Dissertation is brought to you for free and open access by ThinkIR: The University of Louisville's Institutional Repository. It has been accepted for inclusion in Electronic Theses and Dissertations by an authorized administrator of ThinkIR: The University of Louisville's Institutional Repository. This title appears here courtesy of the author, who has retained all other copyrights. For more information, please contact thinkir@louisville.edu. 


\title{
DEVELOPMENT AND APPLICATIONS OF CONDENSED PHASE CAVITY RING-DOWN SPECTROSCOPY FOR STUDIES OF ELECTROCHEMICAL AND INTERFACIAL PROCESSES
}

\author{
By \\ Shadi Ahmad Alnaanah \\ B.S., Muta'h University, 2005 \\ M.S., Muta'h University, 2010 \\ M.S., University of Louisville, 2018
}

\begin{abstract}
A Dissertation Submitted to the Faculty of the College of Arts and Science at the University of Louisville in Partial Fulfillment of the Requirements for the Degree of
\end{abstract}

Doctor of Philosophy in Physics

Department of Physics and Astronomy

University of Louisville

Louisville, Kentucky

December 2020 
Copyright 2020 by Shadi Ahmad Alnaanah

All rights reserved 

DEVELOPMENT AND APPLICATIONS OF CONDENSED PHASE CAVITY RINGDOWN SPECTROSCOPY FOR STUDIES OF ELECTROCHEMICAL AND INTERFACIAL PROCESSES

\author{
By \\ Shadi Ahmad Alnaanah \\ B.S., Muta'h University, 2005 \\ M.S., Muta'h University, 2010 \\ M.S., University of Louisville, 2018
}

A Dissertation Approved on

November 18, 2020

By the Following Dissertation Committee:

\begin{tabular}{c}
\hline $\begin{array}{l}\text { Dr. Sergio B. Mendes } \\
\text { Dissertation Director }\end{array}$ \\
\hline Dr. Jinjun Liu \\
\hline Dr. Serban Smadici \\
\hline Dr. Ming Yu
\end{tabular}

Dr. Ming Yu 


\section{DEDICATION}

This is work wholeheartedly dedicated to my beloved wife, my parents, and my children. 


\section{ACKNOWLEDMENTS}

I would like to take this opportunity to give thanks to all of the people in my life, past and present, who have supported me to complete and succeed in this Ph.D. dissertation. First and for most, I would like to express my deepest gratitude to my research advisors, Professor Sergio B. Mendes for his valuable guidance, motivation and support in completing the research work, patient instruction in reviewing and correcting the manuscript, providing advice and encouragement.

I would also thank all my Ph.D. committee members: Dr. Jinjun Liu, Dr. Serban Smadici, and Dr. Ming Yu.

It was a great privilege and honor to work and cooperate with many people from outside our group. My sincere thank goes to Dr. Thomas J. Roussel for his help with all programming in Labview. My special thank and grateful to Dr. Martin G. O'Toole and Betty M. Nunn, from the Department of Bioengineering, for their help, discussion and valuable comments on the electro-active EW-CRDS bio-sensing applications. I express my special thanks to Dr. Jinjun Liu and Dr. Hamzeh Telfah from the Department of Chemistry for their assistance, support, and suggestions throughout my project.

I would like to say thanks to all past and present friends and research colleagues of photonics research labs at UofL: Dr. Jafar Ghithan, Dr. Aymen Qatamin, Mohammed 
Irziqat, Melanie Dieterlen, Keenan Jones, Brandi Tungett, and David Bloom. Thank you all for sharing those years with me.

I would also like to extend my gratitude to Dr. Jayanthi Chakram, Dr. Chris Davis, Dr. Gamini Sumanasekera, Joshua Rimmer, Mary Gayle, and Missy Klotz from the Department of Physics and Astronomy for their unlimited help.

I am extremely grateful to my parents for their love, prayers, caring and sacrifices for educating and preparing me for my future. In addition, I express my thanks to my brothers, sisters, and the whole family overseas for their love, prayers, and constant support through all the years of my life. Special thank for my brothers in law Bekr, Bader, Odai and Moath Alrofo for their constant support and encouragement during my studies here. I am very much thankful and grateful to my wife, Hasna, who has been my source of inspiration and gave me strength when I thought of giving up. 


\begin{abstract}
DEVELOPMENT AND APPLICATIONS OF CONDENSED PHASE CAVITY RINGDOWN SPECTROSCOPY FOR STUDIES OF ELECTROCHEMICAL AND

INTERFACIAL PROCESSES
\end{abstract}

Shadi Ahmad Alnaanah

November 18, 2020

This dissertation reports the development of ultra-sensitive platforms based on the laser cavity ring-down spectroscopic (CRDS) technique to enable optical and spectroelectrochemical investigations in the condensed phase of matter at challenging scenarios.

Firstly, an electrically-active solid/liquid interface for the evanescent-wave cavity ring-down spectroscopy (EW-CRDS) was developed to specroelectrochemically investigate redox events. By coating the interface of total internal reflection of the EWCRDS platform with a high quality optically transparent and electrically conductive indium tin oxide thin film (ITO), we demonstrated that sufficiently long ring-down times can be achieved to allow for spectroelectrochemical investigations of redox species at solid/liquid interfaces at low surface coverages. The effects of an applied electric potential on the adsorption behavior of a redox protein onto different interfaces were investigated. 
For each interface, the adsorption and desorption constants, the surface equilibrium constant, the Gibbs free energy of adsorption, and the surface coverage were optically measured by our electrically-active EW-CRDS tool. Cyclic voltammetry (CV) scans under synchronous optical readout were performed to study the effects of each molecular interface in the redox process of surface-adsorbed protein species. The electro-active EWCRDS technology is experimentally tested and demonstrated to provide a high performance platform for studies of electrode-driven redox events of surface-confined molecular species at low submonolayer coverages and at a single diffraction-limited spot.

Next, the electrically-active capability of the EW-CRDS device has been extended to develop a bio-sensing strategy based on the combination of the electro-active EW-CRDS platform with a sandwich immunoassay approach for the detection antigens of the influenza A virus (H5N1). Initially, the EW-CRDS was deployed to characterize in-situ and in real-time the formation of the assembly of the immunoassay-based biosensor. Our strategy proceeds in a stepwise manner: in the first step, the surface of the electro-active EW-CRDS device is functionalized with a capture antibody $(\mathrm{Ab})$ aimed at a specific virus antigen. Next, the capture Ab-coated surface is exposed to a target antigen, which after binding to the surface it promotes the immobilization of secondary Ab that has been labeled with a redox-active probe. The redox-active probe methylene blue acts as a transduction element for monitoring molecular binding events and can be electrochemically modulated on the EW-CRDS platform to provide a unique optical interrogation signal. Based on this novel detection strategy, the experimental results have demonstrated an outstanding level of sensitivity in the pico-molar range for detection of the influenza virus antigen. Finally, we used an electrically modulated optical signal collected with an electro-active EW- 
CRDS platform under CV potential modulation for fast detection and direct quantification of a target antigen. Such results demonstrates the potential of the electro-active EW-CRDS technology for high sensitive detection of surface-confined biomarkers at a single spot and at very low concentrations, which open the prospect towards an arrayed-detection technology.

In addition, the highly sensitive CRDS technology in the liquid phase was applied for trace detection of nitrite ions in aqueous environment. The principle of the analytical method used for the determination of trace nitrite is based on the changing of the chromatic reagent color from purple to yellow due to its reduction reaction of nitrite ions in acidic medium, which results in a decrease of optical absorbance. The decrease in absorbance is directly proportional to the nitrite concentration and the CRDS technique was used to measure the absorbance decrease at $532 \mathrm{~nm}$. The experimental results show that nitrite ions could be detected accurately within a low detection limit, which can reach as low as 8 $\mathrm{ng} / \mathrm{ml}$. With the current CRDS setup, we have achieved an absorption sensitivity of about $1 \times 10^{-6} \mathrm{~cm}^{-1}$.

Finally, we have explored the ultra-sensitivity of the CRDS platform for direct measurements in changes of optical loss in ultra-thin films of a semiconductor material (ITO) during surface treatment with ultra-violet (UV) radiation. The results demonstrated the ability of the CRDS to measure minute absorption changes in the ITO ultra-thin film during UV treatment. Upon inserting a glass slide coated with an ITO film at the Brewster's angle inside the optical cavity, the cavity ring-down time of about $1.6 \mu$ s was achieved, which enables measurements of optical absorption loss as low as $3 \times 10^{-6} \mathrm{~cm}^{-1}$. The ITO 
ultra-thin film was treated with UV radiation in two ways: after and during the deposition process at different oxygen flow rates. In order to evaluate the effect of a surface treatment, CRDS platform was employed to measure the extinction coefficient for each coated sample with and without UV exposure, while the change in electrical resistivity was monitored simultaneously. Based on the measurements of optical constants and cavity ring-down times, the UV treatment could be tuned to increase the optical efficiency of an ITO ultrathin film.

These applications are demonstrated to provide novel tools to study the interfacial phenomena with high sensitivity and specificity, which are expected to open several opportunities to investigate a wide range of molecular assemblies and sensing applications. 


\section{TABLE OF CONTENTS}

\section{CONTENTS}

PAGE

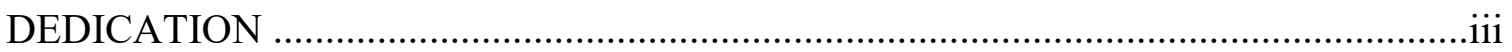

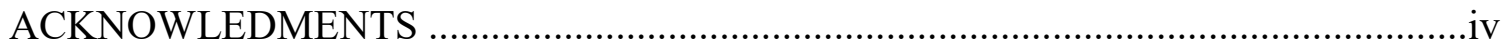

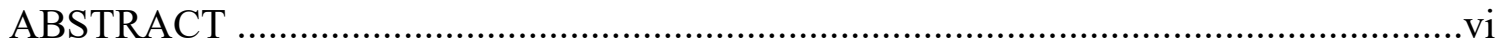

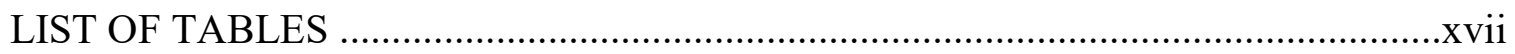

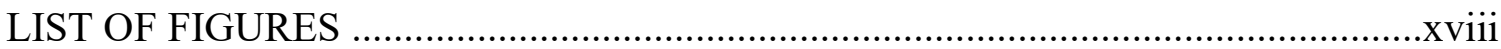

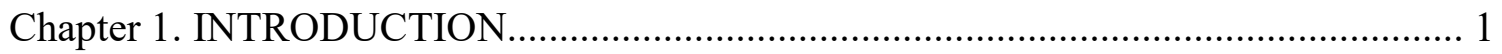

1.1. Importance of Spectroscopy at Surface and Interfaces........................1

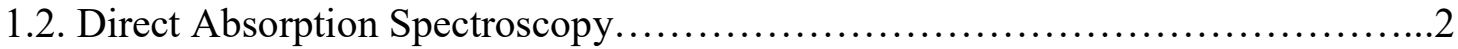

1.3. Cavity Ring-Down Spectroscopy (CRDS) Technique ........................

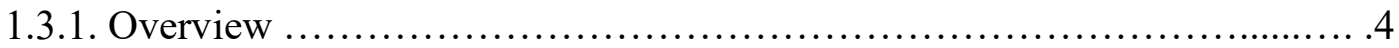

1.3.2. Working Principle of a CRDS Spectrometer............................5

1.3.3. Condensed-Phase CRDS ...........................................

1.3.3.1. Liquid Phase CRDS ............................................ 8 
1.4. Evanescent Wave Cavity Ring-Down Spectroscopy (EW-CRDS).

1.5. Combining EW-CRDS with Electrochemical Techniques..........................13

1.6. Review of EW-CRDS Developments and Applications..........................14

1.6.1. Monitoring molecular Adsorption with EW-CRDS........................14

1.6.2. Biological Systems Interfaces Studies..................................16

1.6.3. Monitoring Electrochemical Processes with EW-CRDS …................18

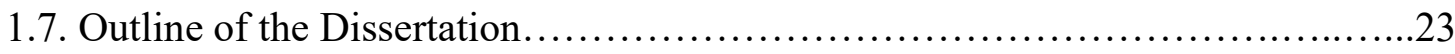

\section{Chapter 2. DESCRIPTION, OPTIMIZATION, AND PERFORMANCE OF A}

CONDENSED PHASE CRDS EXPERIMENTS .............,..............................24

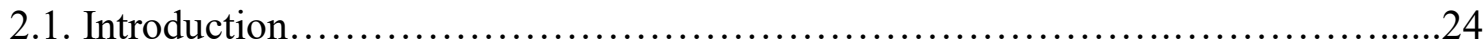

2.2. Cavity Configurations in Condensed Phase CRDS …............................25

2.2.1. Confocal Linear (in-axis) Cavity .......................................25

2.2.2. Brewster's Angle Configuration (off-axis) Cavity...........................26

2.2.3. Thin Film on Brewster's Angle Substrates Configuration...................27

2.2.4. Leaner Cavity with Normal Incidence Cuvette..............................28

2.3. Data Acquisition and Ring-down Time Measurements...............................28

2.3.1. Data Acquisition of EW-CRDS Experiment.................................28

2.3.2. Electrochemistry Data Acquisition........................................... 31 


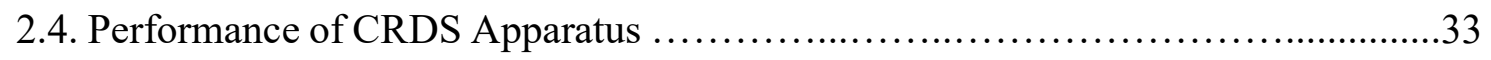

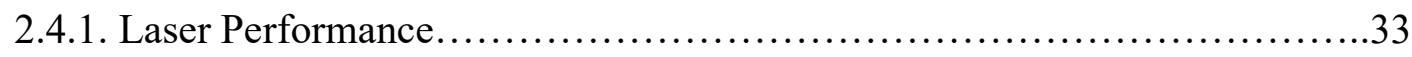

2.4.2. Photomultiplier tube detector performance..................................34

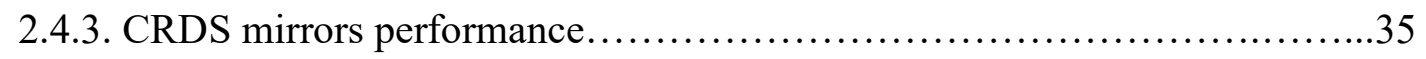

2.5. Calculations of the Optical Loss in EW-CRDS Configurations..........................37

2.6. The Minimum Detectable Absorption coefficient Limit...............................42

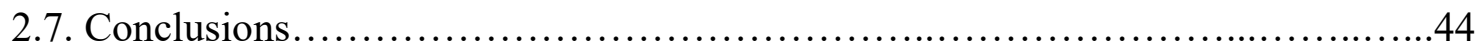

Chapter 3. AN ELECTRO-ACTIVE INTERFACE FOR EVANESCENT-WAVE CAVITY-RING-DOWN SPECTROSCOPY: EXPANDING ITS CAPABILITY FOR ELECTROCHEMICAL AND ADSORPTION KINETIC INVESTIGATIONS..........45

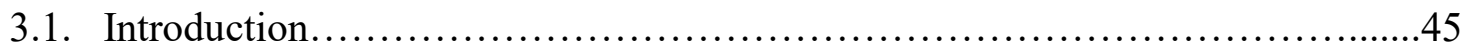

3.2. Probing Redox Reactions of Immobilized Cyt-C Protein ...........................47

3.2.1. EW-CRDS Experimental Setup .....................................47

3.2.2. ITO Ultra-Thin Film Sample Coating.....................................49

3.2.3. Absorbance and Surface Coverage Calculations.........................50

3.2.4. Results and Discussion................................................. 51

3.2.4.1. Determination the Surface Coverage of Adsorbed Cyt-C Protein on

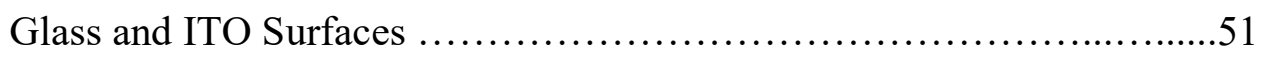

3.2.4.2. Probing Redox Reactions of Immobilized Cyt-C under a Step Potential Modulation.........................................................53 
3.3. Real-Time Monitoring of Adsorption Kinetics under Potential Control and Following the Redox Processes of Cyt-c Protein Using Electro-Active EWCRDS

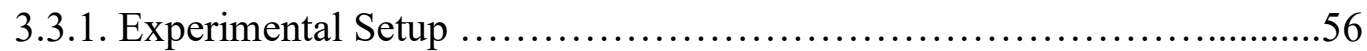

3.3.2. Functionalization Protocols of EW-CRD Surface............................58

3.3.2.1. APTES Functionalization......................................58

3.3.2.2. Primary Cyt-C Capturing Antibody Functionalization..............59

3.3.2.3. Recycling the EW-CRDS Surface.................................60

3.3.3. Binding Kinetics of Cyt-C Protein Model..................................60

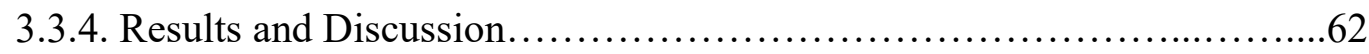

3.3.4.1. EW-CRDS of protein adsorption at Different Surfaces under Open

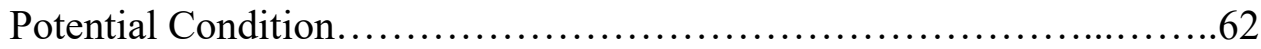

3.3.4.2. Protein Adsorption with the EW-CRDS under ElectricallyControlled Potentials..................................................6

3.3.4.3. Cyclic Voltammetry with the Electro-Active EW-CRDS

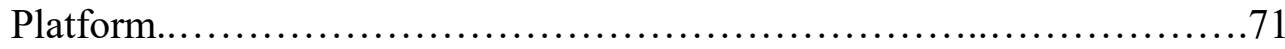

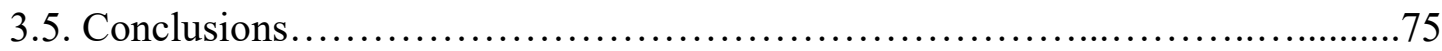

Chapter 4. REAL TIME INFLUENZA VIRUS DETECTION WITH ELECTRO-

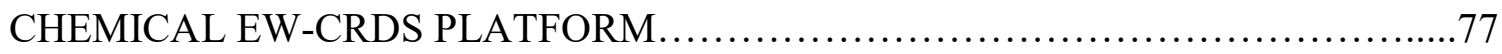

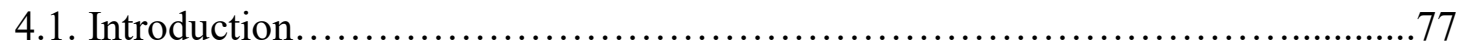

4.2. Principle of Bio-sensing Using EW-CRDS Platform..........................78 
4.3. Experimental section

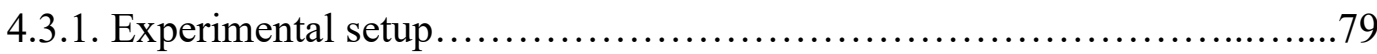

4.3.2. Chemicals and Reagents..................................... 80

4.3.3. Labeling of Secondary Antibody with a Redox Probe......................80

4.3.4. Sensor Functionalization Methods.................................. 82

4.4. Results and Discussions........................................... 83

4.4.1. Real-Time Influenza Virus Immunosensor with EW-CRDS....................83

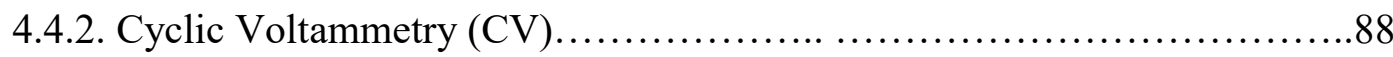

4.5. Conclusions ........................................................91

Chapter 5. HIGHLY SENSITIVE TRACE DETECTION OF NITRITE IONS IN WATER USING LIQUID-PHASE CAVITY RING-DOWN SPECTROSCOPY .................93

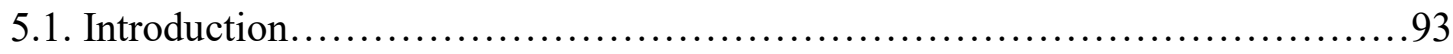

5.2. Principle of nitrite ions detection.................................... 94

5.3. Experimental Section............................................ 96

5.3.1. Experimental setup........................................ 96

5.3.2. Chemicals and Reagents...................................... 97

5.4. Results and Discussion........................................... 97

5.4.1. The effect of sulfuric acid and CV-Ac dye concentration..................97

5.4.2. Nitrite ions detection in water.................................. 100 
5.5. Conclusions.

Chapter 6. CAVITY RING-DOWN SPECTROSCOPY STUDY ON THE UV SURFACE TREATMENT OF ULTRA-THIN INDIUM TIN OXIDE FILM.... 102

6.1. Introduction. 102

6.2. Experimental Section.............................................. 104

6.2.1. ITO ultra-thin film samples preparation ............................ 104

6.2.2. CRDS Experimental Setup....................................... 105

6.2.3. Absorption and Transmittance Measurements of the Thin Film............105

6.2.4. Optical Properties Measurements ..................................107

6.3. Results and Discussions............................................ 109

6.3.1. UV Treatment of ITO Ultra-thin Film after the Sputtering Deposition

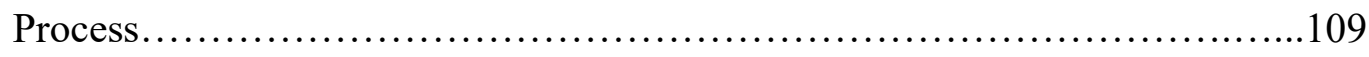

6.3.2. In situ UV Treatment of ITO Ultra-thin Film during the Sputtering Deposition Process..................................................111

6.4. Conclusions...................................................... 114

Chapter 7. SUMMARY AND OUTLOOK.................................... 115

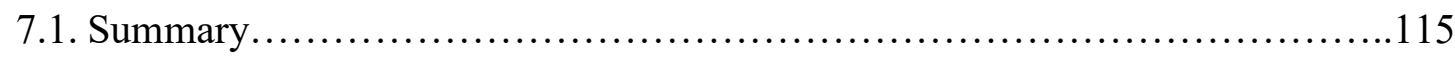

7.2. Outlook....................................................... 117

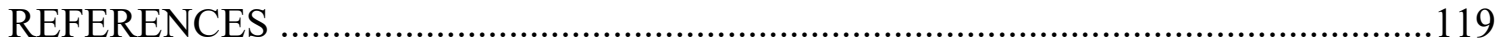

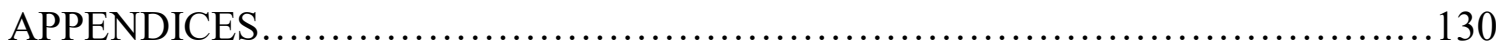


Appendix 1: Labview VI's used for CRDS electrochemistry data acquisition.

Appendix 2: Python program code for ring-down time calculation

Appendix 3: Cyt-C protein samples preparation.

Appendix 4: Binding Stability of the Cyt-C Ab on the APTES surface

Appendix 5: Kinetic Adsorption of Cyt-C Protein onto the APTES and Antibody Surface at Open Potential.

Appendix 6: Kinetic Adsorption of Cyt-C Protein onto the APTES and Antibody Surface at Controlled Potential. 138

Appendix 7: MB-Labeled H5N1 secondary Ab concentration Calculations 140

Appendix 8: Mathematica program code for optical constants calculations of ITO thin film

Appendix 9: The results of the single-pass transmittance and ring-down times measurements of ITO ultra-thin films as coated with and without UV exposure at different oxygen flow rates. 147

Appendix 10: The results of thickness, real and imaginary parts of the refractive index of ITO ultra-thin films as coated with and without UV exposure at different oxygen flow rates .148 


\section{LIST OF TABLES}

TABLE

PAGE

Table 2-1: Typical Surelite Nd-YAG laser (SL II-10) performance and specifications, for

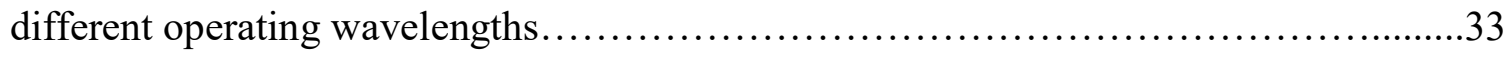

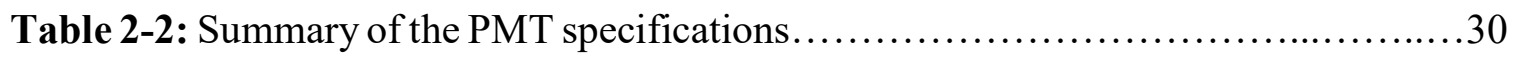

Table 2-3: High reflectivity CRDS mirror specification. .............................

Table 2-4: Comparison of a measured light transmittance, calculated loss coefficient, and ring-down time with two ways: power meter and lock-in amplifier for different EW-CRDS configuration. The measured and calculated ring-down times were compared.

Table 2-5: A minimum detectable absorption coefficient $\left(\Delta \alpha_{\min }\right)$ for various cavity ringdown times of various CRDS configurations. It is clear that $\Delta \alpha_{\min }$ is linearly proportional with ring-down time.

Table 3-1: The Association Rate Constant $\left(\mathrm{k}_{\mathrm{a}}\right)$, the Dissociation Rate Constant $\left(\mathrm{k}_{\mathrm{d}}\right)$, the Equilibrium Constant $\left(\mathrm{k}_{\mathrm{eq}}\right)$, and the Gibbs free energy $(\Delta G)$ for Cyt-C Binding to Different EW-CRDS Functionalized Surfaces...........................................65

Table 3-2: The Equilibrium rate Constant $\left(k_{e q}\right)$ for Cyt-C Binding to different EW-CRDS Functionalized Surfaces with different values of external electric potential............69

Table 4-1: The data of average ring-down times obtained after each washing process of $\mathrm{MB}$-conjugated $\mathrm{Ab}$ in Figure 4-4. . .86

Table 6-1: Real, imaginary parts of refractive index, and thickness of ITO ultra-thin film samples without and with UV treatment. .110 


\section{LIST OF FIGURES}

FIGURE

PAGE

Figure 1-1: Schematic representation of single-pass direct absorption spectroscopy. The sample is contained in the absorption cell with length 1 . The transmission of the light through the cell is measured as a function of the cell length and the wavelength. 3

Figure 1-2: Schematic of the description of CRDS spectrometer. After abrupt termination of the excitation, the light stored in the cavity decays exponentially as it bounces back and forth between the mirrors, losing some intensity at each reflection. The decay rate of the light can be measured behind the cavity ....................................... 5

Figure 1-3: Schematic figures for different modes of evanescent-wave absorption spectroscopy. (a) Attenuated total reflectance absorption spectroscopy. (b) Waveguide absorption spectroscopy. (c) Evanescent-wave cavity ring-down spectroscopy..........12

Figure 1-4: (a) Schematic representation of the thin-layer cell for EW-CRDS showing the generation of $\mathrm{Fe}(\mathrm{CN})_{6}{ }^{3-}$ from $\mathrm{Fe}(\mathrm{CN})_{6}{ }^{4-}$. (b) Ferricyanide concentration and the generated current, as a function of time, recorded during the $\mathrm{CV}$ scan $(5 \mathrm{mV} / \mathrm{s})$. The red (concentration) and green (CV) solid lines are simulations based on finite element modelling of a reversible process............................................. 18

Figure 1-5: (a) Scheme of the thin layer electrochemical cell to dissolve the Ag NPs by electrochemically generation of $\operatorname{Ir}(\mathrm{IV})$ from $\operatorname{Ir}(\mathrm{III})$ by stepping the potential for $10 \mathrm{~s}$. (b) Absorbance transients (black line) and theoretical fit (red line) for a typical dissolution

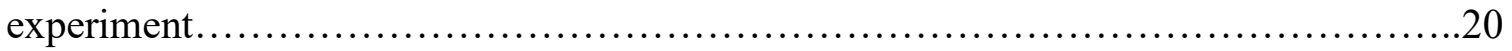

Figure 1-6: (a) Schematic representation of the thin-layer cell was used to measure the electron transfer constant during the reduction of immobilized $\mathrm{Cyt}-\mathrm{C}$ by a redox mediator. (b) The optical signal response of EW-CRDS device at $400 \mathrm{~nm}$, as a function of time during a potential step........................................................... 21 
Figure 2-1: Schematic representation of the confocal linear cavity employing coated rightangle prism. In a confocal geometry the radii of curvature of the two mirrors $\left(R_{1}\right.$ and $\left.R_{2}\right)$ should equal the cavity length (L)..... .25

Figure 2-2: Schematic representation of a Brewster's angle cavity employing uncoated right-angle prism. The laser beam hits the prism surface close to the edge at Brewster's angle.

Figure 2-3: Schematic representation of a thin film CRDS. The glass substrate is placed inside the optical cavity in such way that the laser beam enters and leaves the substrate at Brewster's angle....

Figure 2-4: Schematic representation of liquid phase CRDS, the light enters and leaves the linear cavity perpendicular to the planar sides of the quartz cuvette.....................28

Figure 2-5: Experimental single-shot cavity-ring-down trace obtained with the Brewster's angle EW-CRDS setup. The red line is an exponential decay fit to the data....

Figure 2-6: Screenshot of the Labview front panel used for EW-CRDS data acquisition. (a)sitting the oscilloscope parameters and plot of CRD signal as a function of time, (b) exponential decay function fit, and (c) sub-VI used to plot and save ring-down time as a function of time. .............................................................. 30

Figure 2-7: Screenshot of the front panel of the LabVIEW program used for spectroelectrochemical experiment data acquisition. (a) Settings the oscilloscope and CV experiments parameters tap, (b) Start switch for performing an electrochemical experiment (CV) and time stamps for data synchronization, (c) plot of the electric current as a function of applied potential in CV scan, (d) plot of applied voltage as a function of time, (e) EWCRDS signal as a function of time, (f) exponential decay fit of EW-CRDS signal, (g) extracted electric potential values................................................... 32

Figure 2-8: Measured mirror reflectivity vs. normalized wavelength for $532 \mathrm{~nm}$ CRDS optics dielectric mirrors forming the cavity...................................... 36

Figure 2-9: The experimental setup for a single-pass transmittance measurement through the prism. CW laser was modulated using a mechanical chopper, the laser polarization was chosen using a linear polarizer and half-wave plate. The reference signal intensity was detected by the photo detector (PMT1), while, the incident $\left(I_{l}\right)$ and the transmitted $\left(I_{2}\right)$ light intensities were measured by PMT2. The optical signal was amplified using current preamplifier and was fed to the lock-in amplifier. 39 
Figure 3-1: (a) Schematic diagram of the experimental setup for the EW-CRDS. (b) Enlarged picture of electrochemical flow cell and prism.

Figure 3-2: Adsorption isotherm of Cyt-C on ITO surface at $\mathrm{pH}-7$, measured at $532 \mathrm{~nm}$. The dashed curve is the best fit of the experimental data to a Langmuir adsorption model. .52

Figure 3-3: Absorbance data (black data points) as measured by the EW-CRDS platform at $532 \mathrm{~nm}$ for an adsorbed sub-monolayer of Cyt-C protein undergoing step potential modulation (from $-0.5 \mathrm{~V}$ to $0.5 \mathrm{~V}$ )on ITO surface, with a curve fitting (red curve) and the derivative of the fitted line (blue curve) to determine the formal potential (here the blue

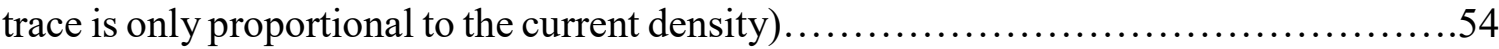

Figure 3-4: Schematic diagram of experimental setup for EW-CRDS measurement. The spectro-electrochemical flow cell attached to the TIR surface. .56

Figure 3-5: Confocal image of the ITO/APTES EW-CRDS surface functionalized with fluorescent Alexa labeled Cyt-C-Ab, for different APTES functionalization time (a) 1h, (b) $3 h,(c) 6 h$

Figure 3-6: (a) Surface coverage change of Cyt-C binding on the bare ITO measured by EW-CRDS platform for different concentrations in real-time. (b) The plot of the binding rate versus bounded surface coverage for different Cyt-C concentrations. (c) The plot of the slope values was obtained in Figure 3-6(b) and plotted against Cyt-C concentration, to determine the association and dissociation constants

Figure 3-7: (a) Equilibrium rate constant $\left(\mathrm{k}_{\mathrm{eq}}\right)$, and (b) Gibbs free energy, for Cyt-C binding to different EW-CRDS functionalized surfaces, at open-potential condition....

Figure 3-8: Surface coverage vs time for different concentrations of $\mathrm{Cyt}-\mathrm{C}$ with different values of an external applied potential on bare ITO surface... 68

Figure 3-9: (a) The values of adsorption equilibrium constant, and (b) Gibbs free energy, versus applied electric potential $(-0.4,-0.2,0.2$, and $0.4 \mathrm{~V})$ for Cyt-C binding to different EW-CRDS functionalized surfaces .70

Figure 3-10: Absorbance of Cyt-C plotted against electric potential at each functionalized surface of the EW-CRDS interface. The bulk concentration of Cyt-C protein was kept constant at $500 \mathrm{nM}$ and the $\mathrm{CV}$ potential modulation parameters where all the same: range of $-0.3 \mathrm{~V}$ to $+0.6 \mathrm{~V}$ .72 
Figure 3-11: (a) The measured total surface coverage density of Cyt-C in its oxidized form at different interfaces. (b) The fraction for each interface of surface density of reduced species over the total surface density in the oxidized form.......................... 74

Figure 4-1: Schematic illustration of a sandwich bioassay strategy using electro-active EW-CRDS platform. The steps of fabrication biosensor involve: the functionalization of the surface with chemical group to promote the adsorption of capture $\mathrm{Ab}$, binding the target analyte, and finally binding the secondary $\mathrm{Ab}$ with a redox-active optical

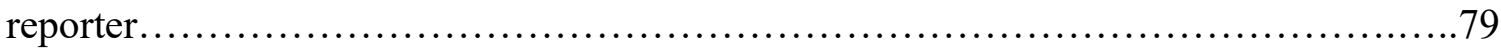

Figure 4-2: UV Vis spectra of oxidized (red curve) and reduced form (green curve) of MB. $\mathrm{MB}$ was reduced chemically by using ascorbic acid $22 \mathrm{mM}$ in the presence of $500 \mathrm{mM}$ $\mathrm{HCl}$.

Figure 4-3: UV Vis absorption spectra of free MB ((blue curve) and secondary H5N1 Ab (red curve), the shift in the two spectra is evidence of successfully aimed conjugation............................................................. 82

Figure 4-4: Influenza virus immunosensor measured on EW-CRDS platform. The ringdown time recorded as a function of time during the Steps of the sandwich immunoassay assembly (see text and Table 2). At time ' $a$ ' the capture Ab was introduce to the flow cell, at time ' $\mathrm{I}$ ' BSA was added, at the times indicated by ' $b$ ', 'c', ' $d$ ', ' $\mathrm{f}$ ', and $g$ virus intigen was added with concentrations $0,20,50,100$, and $200 \mathrm{ng} / \mathrm{ml}$, respectively. The numbers indicated by 'II', 'IV', 'V', and 'VI' are the times when the MB-labeled Ab were added after each antigen concentration. The number indicated by 1, 2, 3, 4, 5, 6, and 7 are the times when the flow cell was rinsed with $200 \mu$ of PBS. .84

Figure 4-5: Schematic representation of the results in the Figures 4-4 in case (a) When the antigen is absent (b) In the presence of the antigen, MB-labeled H5N1 Ab is adsorbed to the surface. The BSA worked to block the nonspecific adsorption sites. . .85

Figure 4-6: The calibration curve of the optical absorbance measured by EW-CRDS for each concentration of virus antigen, which allows determining the limit of detection.....87

Figure 4-7: The optical signal response at $532 \mathrm{~nm}$ as measured by the EW-CRDS platform under $\mathrm{CV}$ potential modulation. Where the red trace represents the optical absorbance in the presence of HA virus antigen. The black trace when the virus antigen is not present and the probe species are not bound to the surface; the analytical signal vanishes after the flowcell was rinsed. 
Figure 4-8: (Black trace) Modulated absorbance at $532 \mathrm{~nm}$ from the data presented in Figures 4-7 with a curve fitting (red trace) and the current density corresponding to the fitted line (blue curve) to determine the formal potential.

Figure 5-1: (a) The observed color change from purple to yellow due to the reduction of $\mathrm{CV}$-Ac with nitrite ions in acidic medium. (b) UV-Visible spectra of CV-Ac and its reduced form in the wavelength range of $300-800 \mathrm{~nm}$. The optimum conditions: $5 \mu \mathrm{M} \mathrm{CV}-$ Ac, $2 \mathrm{M} \mathrm{H}_{2} \mathrm{SO}_{4}, 1 \mu \mathrm{g} / \mathrm{ml} \mathrm{NO}{ }^{2-}$ and $15 \mathrm{~min}$ at room temperature .95

Figure 5-2: Schematic representation of the liquid phase CRDS setup. The quartz cuvette was inserted under normal incidence inside the optical cavity. .96

Figure 5-3: The reduction of CV-Ac dye with nitrite ions was optically monitored in real time by CRDS tool. .98

Figure 5-4: Effect of sulfuric acid concentration on the change of absorbance during the reduction of $\mathrm{CV}$-Ac dye with nitrite ions. Where the $\mathrm{CV}$-Ac concertation was $5 \mu \mathrm{M}$ and the nitrite ion of $1 \mu \mathrm{g} / \mathrm{ml} \mathrm{L}$

Figure 5-5: The change in the optical absorbance against various concentrations of nitrite ions at the wavelength $532 \mathrm{~nm}$. The inset is the calibration graph displayed a good linear relationship between the change in absorbance and the $\mathrm{NO}^{2-}$ concentration in the range from 0.05 to $100 \mathrm{ng} / \mathrm{ml}$. The optimal concentrations of $\mathrm{CV}$-Ac dye and $\mathrm{H}_{2} \mathrm{SO}_{4}$ acid were 5 $\mu \mathrm{M}$ and $1 \mathrm{M}$, respectively.

Figure 6-1: Scheme of the thin film CRDS experimental setup.

Figure 6-2: Schematic representation of the experimental setup used to measure the transmittance and reflectance versus the incident angle, for s and p-polarized light, of ITO ultra-thin film.

Figure 6-3: Reflectance and transmittance against incident angle for $\mathrm{p}, \mathrm{s}$ polarized light. The black dots are experimental results, and the red solid line is the modulation data. ... 108

Figure 6-4: (a) Ring-down time data as measured by CRDS tool for ITO ultra-thin film with and without UV treatment, (b) Absorbance per pass in the cavity as a function of total time during UV treatment................................................. 110

Figure 6-5: The variation of the electrical resistivity of ITO ultra-thin film in real time during UV treatment. 
Figure 6-6: The change in (a) ring-down time, (b) extinction coefficient for ITO films as deposited with and without UV exposure at different oxygen flow rates................112

Figure 6-7: Electrical resistivity of ITO ultra-thin film samples deposited with and without UV exposure, under different oxygen flow rates.............................. 113 


\section{CHAPTER 1}

INTRODUCTION

\subsection{Importance of Spectroscopy at Surface and Interfaces}

The science of surface and interfaces is one of the most challenging areas for physicists and chemists and it is fundamental in many areas of science and technology [13]. The recent applications in modern technologies involve new materials and processes, for example, interactions between biological molecules and surfaces [4, 5], development of microelectronics [6], heterogonous catalysis [7], and molecular electrochemistry [8, 9], in which interfaces play a crucial role.

Spectroscopy of interfaces is important to understand many interfacial processes, such as the mechanism of proteins adsorption and orientation, molecular surface electrochemistry on thin film electrode, and electrochemical biosensing. The main challenge in the spectroscopy of surface and interface science is that the analytical signal is very weak due to the extremely low concentration of the molecules at the surface compared with those in the bulk solution. Therefore, the finding of sensitive analytical tools is necessary to investigate the processes that occur in that region. 


\subsection{Direct Absorption Spectroscopy}

Absorption spectroscopy, in the UV, visible and IR range of the electromagnetic spectrum is the most ubiquitous and accurate methods available to physicists and chemists. It can be applied both in the gaseous and liquid phase and to both atoms and molecules. Unlike other spectroscopic techniques such as fluorescence spectroscopy or resonantly enhanced multi-photon ionization (REMPI ), where the molecule should be fluorescent or ignitable, an absorption spectrum can be measured for any molecule $[10,11]$.

In conventional absorption spectrometer as shown in Figure 1.1, the attenuation of light passing the sample is measured by comparing the intensity of light transmitted through a sample $(I(l, \lambda))$ with the initial intensity of light beam $\left(I_{0}\right)$. Thus, the absorbed light by the sample is then given by:

$\Delta I=I_{0}-I(l, \lambda)$

In addition, it can be described by the Beer-Lambert law as:

$\Delta I=\log \frac{I(l, \lambda)}{I_{0}}=\varepsilon_{\lambda} C l$

Where $\varepsilon_{\lambda}$ is the wavelength dependent absorption coefficient, $\mathrm{C}$ is the concentration of the absorbing species, and $l$ is the path length through the sample.

Increasing the sensitivity of absorption spectroscopy is the main challenge in many conventional absorption techniques. In Equation1-2, the dimensionless outcome $\left(\varepsilon_{\lambda} C l\right)$ of a conventional absorption measurement demonstrates limitations of conventional 


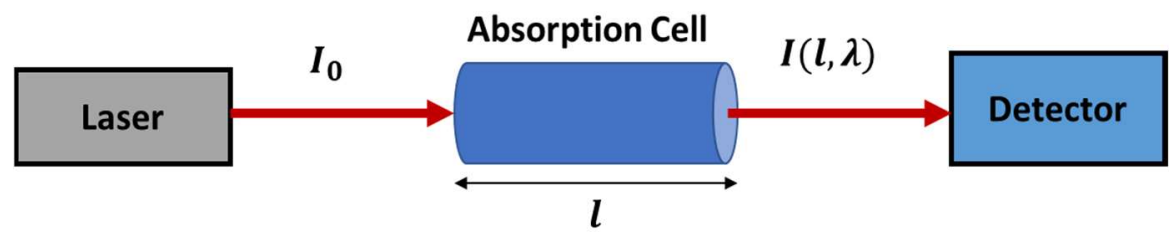

Figure 1-1: Schematic representation of single-pass direct absorption spectroscopy. The sample is contained in the absorption cell with length 1 . The transmission of the light through the cell is measured as a function of the cell length and the wavelength.

absorption techniques, which result in limiting the sensitivity; for low analyte concentrations, weak absorbers, or short path length, both $I_{0}$ and $I(l, \lambda)$ are relatively large, so a small intensity difference $\Delta I$ is measured on a large background $[12,13]$. Thus, conventional absorption measurements are highly dependent on the intensity stability of the light source and detector used. Furthermore, the low sensitivity is the main drawback in many of the conventional absorption spectroscopy techniques such as single transmittance and reflectance optical probing. The lack of sensitivity in those methods is due to the one-time interaction between the probing light beam and the molecules. Several alternative methods have been developed to overcome the lack of sensitivity. In this chapter, one of these alternatives, cavity ring-down spectroscopy (CRDS) is described in detail. 


\subsection{Cavity Ring-Down Spectroscopy Technique}

\subsubsection{Overview}

CRDS is a sensitive absorption technique in which sub-ppb absorbance can be measured without the need for long path lengths through the sample. CRDS based on the measurement of the photon decay time measured behind a high-finesse optical resonator. It has significant advantages over other conventional absorbance spectroscopy. Firstly, the effective absorption path length is very long (which can be in the range of $10 \mathrm{~km}$, for $10^{4}$ passes through a $1 \mathrm{~m}$ cavity length) due to the multi-pass through the sample of interest, so extremely low concentration detection limits are feasible. Secondly, by deploying a single light pulse in each measurement of the photon lifetime, many detrimental effects associated with intensity fluctuations, which is invariably present in any laser light source, are largely eliminated [14-16]. Early in 1980, the cavity enhanced spectroscopy was initially used by Herbelin et al. to measure the reflectance of highly reflective mirrors [17]. In 1984, Anderson et al. used a continuous wave (CW) HeNe laser to measure the photon lifetime within the optical cavity [18]. CRDS absorption technique was first introduced to do spectroscopic measurements by O' Keefe and Deacon in 1988 [19]; with a pulsed dye laser and simple CRDS setup they were able to measure optical absorption spectra of gases.

In the following sections, the CRDS technique is described, and the extension of the technique to sensitive condensed phase spectroscopic measurements is briefly reviewed. 


\subsubsection{Working Principle of a CRDS Spectrometer}

CRDS technique, in its simplest form, is schematically shown in Figure1-1. The optical cavity of high quality-factor which is typically obtained by high-quality mirrors (with reflectivity (R) on the order of $99.99 \%$ ) and accurate alignment that will then sustain the pulse inside the cavity for a long time (typically in the order of $\mu$ s). By considering the loss (1-R) at each reflection, the minute intensity of the light pulse that escapes the optical cavity $\left(I_{t}\right)$ decreases monotonically at each round trip and creates an exponentially decaying trace of the light intensity against time. The separation between adjacent pulses is equal to the round trip time $\left(t_{r}=2 L / c\right)$.

The time required for the light intensity to decrease to $1 / \mathrm{e}$ of its initial value is typically named the cavity ring-down time, also known as the photon lifetime.

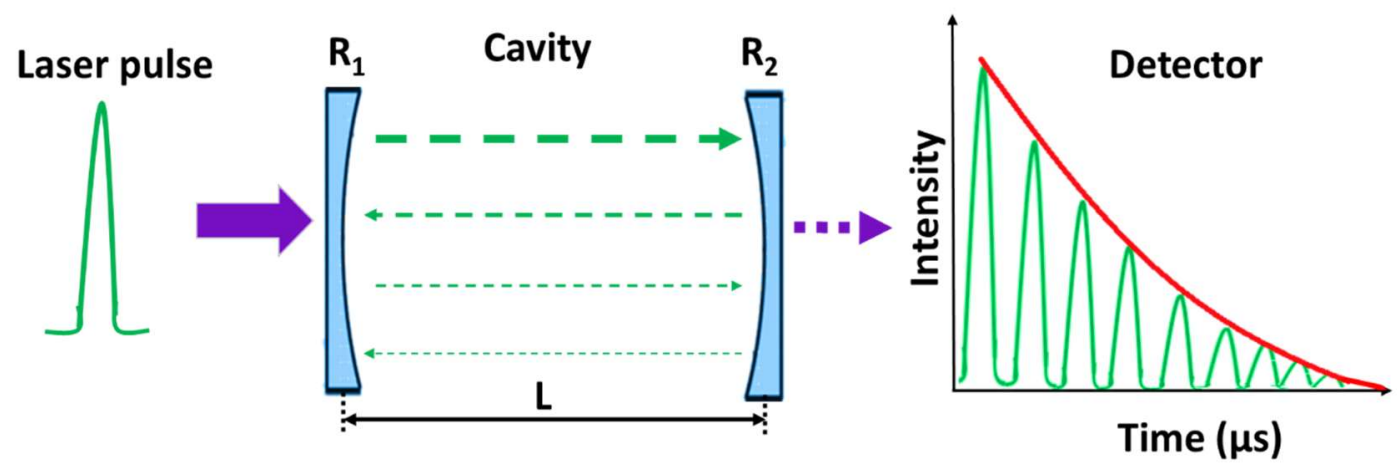

Figure 1-2: Schematic of the description of CRDS spectrometer. After abrupt termination of the excitation, the light stored in the cavity decays exponentially as it bounces back and forth between the mirrors, losing some intensity at each reflection. The decay rate of the light can be measured behind the cavity. 
A detector placed behind the second mirror records the decay in the intensity of light $\left(I_{t}\right)$ exiting the cavity over time $(\mathrm{t})$.

$I_{t}=I_{0} e^{-\frac{t}{\tau}}$

where $I_{0}$ is the light intensity incident upon the first cavity mirror. The ring-down time $(\tau)$ is related to the reflection, scatter $\left(\mathcal{L}_{\text {scatt }}\right)$ losses, and the absorption coefficient $(\alpha)$ of the chemical species between the mirrors as following:

$\tau=\frac{n_{\text {avg }} L}{c\left[(1-R)+(\alpha l)+\mathcal{L}_{\text {scatt }}\right]}$

where $L$ is the cavity length, $l$ is the effective path length through the sample (in the gas phase this is often equivalent to $L$ ), $c$ is the speed of light and $n_{\text {avg }}$ is the weighted refractive index of the cavity if it contains more than a single medium [20].

If there is no absorbing or scattering material in the cavity, Equation 1-2 reduces to:

$$
\tau_{0}=\frac{L}{c(1-R)}
$$

As we notice in Equation 1-5, the ring-down time $\left(\tau_{0}\right)$ is an intrinsic property of a cavity, only depending on the length of the cavity and the reflectivity of the mirrors.

In the presence of an absorber, the absorbance per pass through the sample can be directly derived from the measured ring-down time via:

$$
A=\frac{n_{\text {avg } L}}{\ln (10) c}\left[\frac{1}{\tau}-\frac{1}{\tau_{0}}\right]
$$


Where $\tau$ and $\tau_{0}$ are the ring-down times in the presence and absence of absorber, respectively. Subtraction the base line is necessary to eliminate any reflection and scattering losses.

In practice, the sensitivity of most pulsed CRDS apparatus is limited substantially by poor mode matching of the pulsed laser beam to the fundamental transverse $\left(\mathrm{TEM}_{0,0}\right)[21$ 23]. In addition, compared to CW-CRDS, the high spectral resolution and high sensitivity of pulsed laser CRDS can be achieved by designing sophisticated experimental schemes $[15,24]$.

The CRDS technique has a significant advantage over conventional spectroscopy techniques, where the measurement of ring-down time in the time domain is intrinsically more accurate than observing small intensity changes on top of the strong laser pulse background. Therefore, unlike most pulsed, direct absorption methods, the cavity ringdown is insensitive to the fluctuation in the intensity of the light source [15].

For a typical CRDS experimental setup $L=1 \mathrm{~m}, \mathrm{R}=99.995 \%$, the empty-cavity ring-down time is $66 \mu$ s and the effective path length is $20 \mathrm{~km}$.

\subsubsection{Condensed Phase CRDS}

While CRDS technique has been applied several decades ago and became a standard technique in gas phase. The implementation of CRDS in condensed phase is challenging for two reasons: (I) the higher extinctions of solvent or molecules inside the cavity and (II) inserting any component like glass substrate or cuvette leads to a strong reflection and the Raleigh scattering losses, which increase linearly with path length, so no longer that 
increasing the path length increase the sensitivity gain. This has led to a novel design of the cavity [25].

\subsubsection{Liquid Phase CRDS}

In liquid phase, the use of additional surfaces can be eliminated by placing the liquid directly in the entire cavity (liquid-filled cavity), so the cavity mirrors are directly in contact with the liquid [26-28]. Early studies using a liquid-filled cavity Hallock et al. obtained excellent results with a baseline noise of $10^{-6} \mathrm{~cm}^{-1}$ and the uncertainty on the ring-down time of $358 \mathrm{~ns}$ was only $1.7 \mathrm{~ns}$, but a large sample volume was consumed (as large as tens of ml) [26]. In later studies liquid- filled cavity has been deployed for studying the reduction kinetics of methylene blue by ascorbic acid [27]. Previous studies revealed that contact between the mirrors and liquid shouldn't cause any degradation in the mirror's reflectivity and the effects of stirring or applying a constant flow of liquid did not affect the measurements [26].

As an alternative of the liquid-filled cavity and to prevent the direct contact with the mirror, Alexander et al. deployed a flowing liquid sheet-jet in a linear cavity to introduce liquids into a cavity. The results revealed a baseline noise level as low as $0.5 \times 10^{-7}$ A.U. resulting in a $71 \mathrm{nM}$ limit of detection of a good absorber in a flow of $3.4 \mathrm{ml} \mathrm{s}^{-1}$. However, this method is not favorable for analytical measurements, because a high flow rates and viscosity are needed to create a stable liquid flow, as well as the unfavorable optical pathlength through the jet [29]. 
A third possible configuration is aligning a cuvette under $90 \circ$ in such a way that reflection losses are maintained in the cavity. Fiedler et al. deployed a not parallel output broadband light source, the reason for choosing this configuration is obvious, since the deferent rays with different angles of incident, it is difficult to define one Brewster's angle and the 0-degree geometry yields lower optical losses [30].

Alternatively, a Placing of cuvette close to Brewster's angle in the cavity, which minimize reflections loss, thereby enhancing sensitivity. In the first demonstration of Liquid phase CRDS under Brewster's angle in a linear cavity, Xu et al. recorded the absorption spectra of the fifth overtone of the $\mathrm{C}-\mathrm{H}$ stretch vibration of benzene, with excellent sensitivity compared with other conventional absorbance spectroscopy. Double standard quartz cuvette was placed at Brewster's angle; the ring-down time with the single quartz cell filled by pure hexane of about $0.8 \mu$ s was reported [31].

CRDS detection can also be used for studying the reaction kinetics of short-lived species, such as the $\mathrm{NO}_{3}$ radical, with terpenes in solution. In this study, nitrate radicals were photoinduced by photolysis inside a cuvette placed under Brewster's angle in a linear cavity. At the same time, the reaction of the nitrate radicals and monoterpenes was followed online by monitoring the absorbance decrease [32].

\subsubsection{Solid Phase CRDS}

For solid state CRDS, thin films can be deposited on substrates that are placed inside the cavity at different geometries. The first demonstration of a solid state CRDS was published by Engeln et al. In this work an uncoated BK7 window was inserted in a linear 
cavity at normal incident geometry to obtain a ring-down time in order of $9 \mu$ s for empty cavity, but the ring-down time decreased to about 700 ns upon insertion BK7 window; during this time the light passed through the sample about 1100 times which offer a good sensitivity. The polarization dependent CRDS was used to measure the Verdet constant for the BK7 window, which describes the Faraday effect or the strength of the optical activity induced by a magnetic field [33]. The same group used a similar configuration to measure sensitively fundamental IR lines of a thin $\mathrm{C}_{60}(20-30 \mathrm{~nm})$ film deposited at $\mathrm{ZnSe}$ window [34]. Egashira et al. used the single-mode CRDS to measure an infrared spectrum of the C-H stretching vibration of alkylsiloxane monolayer films grafted on a silicon substrate placed under normal incidence. The measured absorbance was position dependent; it was maximized at the antinodes of the standing wave in the cavity, whereas it was absent at the nodes [35]. Logunov et al. used CRDS to probe relatively thin $(2-20 \mu \mathrm{m})$ polymer film samples in the telecommunication wavelength ranges of $1200-1650 \mathrm{~nm}$ to study the properties of thin films compared to thick bulk films [36]. Marcus et al. demonstrated the ability of the CRDS technique in mid-infrared spectroscopy to sensitively record very small optical loss of thin film; with a Brewster's angle configuration they recorded loss in a $\mathrm{C}_{60}$ film on a $\mathrm{BaF}_{2}$ substrate with absorption sensitivities as small as $1 \times 10^{-7}[37]$.

\subsection{Evanescent-Wave Cavity Ring-down Spectroscopy (EW-CRDS)}

EW-CRDS technique was developed originally in 1997 [38]. It combines the high sensitivity of CRDS absorption measurements with the specially localized evanescent wave (EW) spectroscopic technique, which make the technique highly specific for absorption measurements at solid/liquid interfaces. In EW-CRDS, upon insertion the prism 
in the center of the cavity, the laser beam passing through the prism undergoes total internal reflection (TIR), at the position of TIR, an evanescent field is established whose amplitude decays exponentially with distance from the boundary and penetrates a short distance (typically a few hundred nanometers). As a result, only molecules that are adsorbed at, or are near the surface are detected.

EW-CRDS has three main advantages: Firstly, as illustrated in Figure 1-3(a), it is highly sensitive due to the time-sequence of multiple bounces at a particular spot on the sample of interest. A high quality resonator can reach up to several hundred sequential bounces on a particular diffraction-limited spot on the sample, which highly increases the light-matter interaction and the analytical signal of interest. Secondly, it is spatially localized at the position of total internal reflection of the light beam repeatedly probing a multiple spots over a small footprint near the surface.

This particular feature is in sharp contrast to the ATR geometries, as shown in Figure 13(b), where at the positions of the multiple total internal reflection (TIR), multiple EW formed to enhance the analytical signal. Similarly for single-mode or multi-mode optical waveguide spectroscopy, as shown in Figure 1-3(c), where the high sensitivity is achieved through the light beam propagating over a long path and sampling a substantially larger (many times heterogeneous) volume of the sample. Thirdly, by deploying a single light pulse in each measurement of the photon lifetime, many detrimental effects associated with intensity fluctuations invariably present in any laser light source are largely eliminated [20, $39-41]$. 

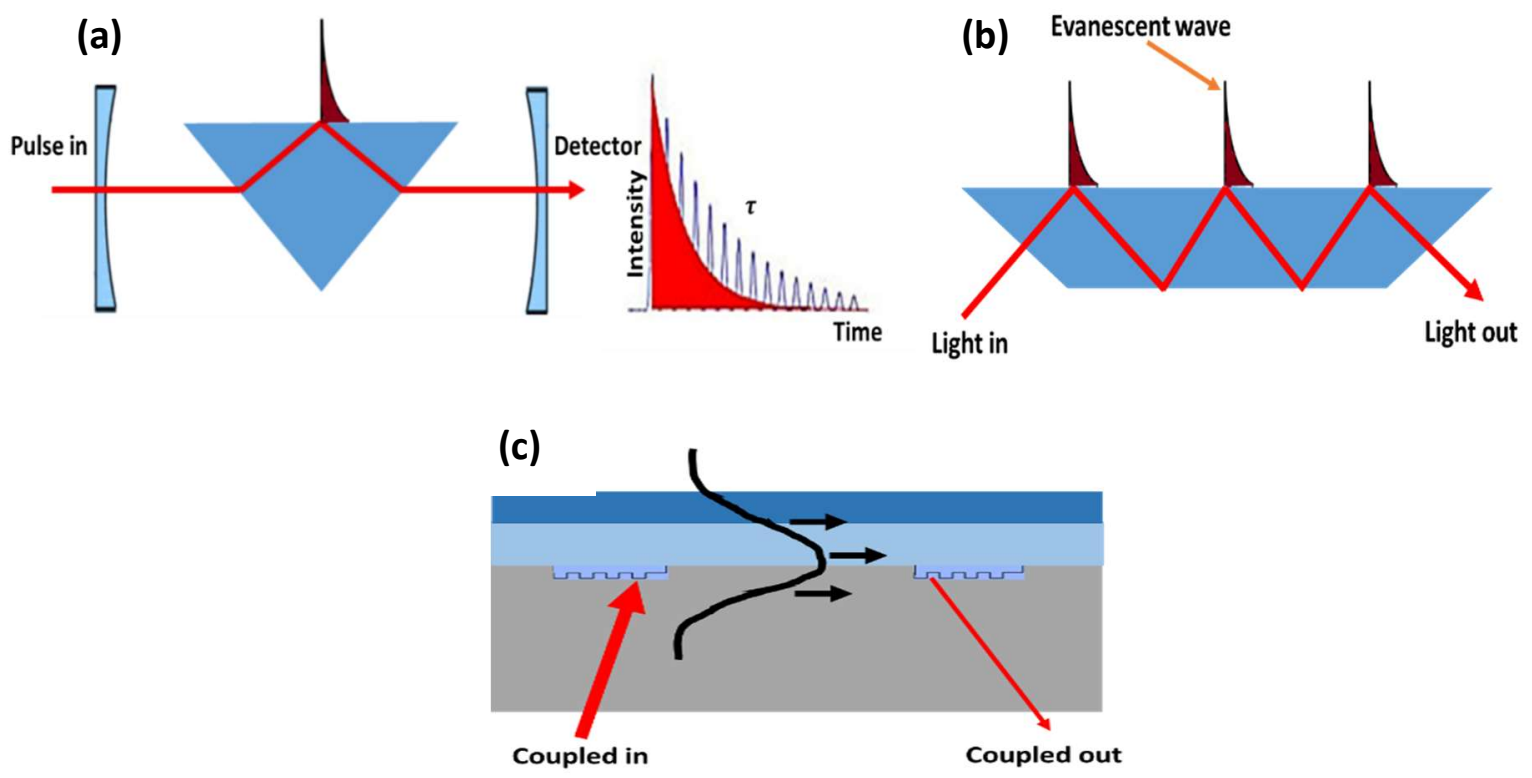

Figure 1-3: Schematic figures for different modes of evanescent-wave absorption spectroscopy. (a) Evanescent-wave cavity ring-down spectroscopy. (b) Attenuated total reflectance absorption spectroscopy. (c) Single-mode Waveguide absorption

During the TIR, the light circulating within the cavity will be sensitive to absorption or scattering losses within the evanescent field established within the solution. The penetration depth of the evanescent field $d_{p}$ can be calculated according to:

$d_{p}=\frac{\lambda}{2 \pi \sqrt{\left(n_{1}^{2} \sin ^{2} \theta-n_{2}^{2}\right)}}$

Where $\lambda$ is the wavelength of the light source, $\theta$ the angle of incidence, and $n_{1}$ and $n_{2}$ are the refractive indices of the prism and the solution, respectively. $d_{p}$ is a fraction of light wavelength and it typically in the range of 50-500nm [42]. 
In fact, the total thickness of the sample probed by the evanescent field is much greater than the $d_{p}$. The bulk absorbance in Equation 1.3 can be written as $A=\alpha d_{e}$, where $d_{e}$ is the effective depth or optical path length. In literature $d_{e}$ is commonly used to compare absorbance in transmission mode with those obtained with an ATR geometry, and it can be defined as the thickness of solution or film which gives the same optical absorbance in conventional transmission measurement at normal incidence. For ppolarized (in the plane of the beam and the surface normal) light, the effective depth is given by $[14,42,43]$ :

$d_{e}=\frac{n_{12} \lambda\left(2 \sin ^{2} \theta-n_{21}^{2}\right) \cos \theta}{n_{1} \pi\left(1-n_{21}^{2}\right)\left[\left(1+n_{21}{ }^{2}\right) \sin ^{2} \theta-n_{21}^{2}\right]\left(\sin ^{2} \theta-n_{21}^{2}{ }^{2}\right)^{1 / 2}}$

where $\theta$ is the angle of incidence, $\lambda$ is the wavelength of the light, and $n_{21}=n_{2} / n_{1}, n_{1}$ and $n_{2}$ are the refractive indices of the prism glass and the liquid medium.

\subsection{Combining EW-CRDS with Electrochemical Techniques}

The combination of electrochemical with spectroscopic techniques is known as spectroelectrochemical methods. In this methods, the optical signal is spectrally tuned (by introducing the working electrode at solid/liquid interface) to probe faradaic electric current of redox activities of adsorbed redox species. Such electro-optical combination are important to an increase the sensing sensitivity of the specific surface binding molecules, this selectivity is important to avoid a non-faradaic components from molecules in the bulk solution that invariably produce non-negligible background signals. Many 
spectroelectrochemical techniques have been developed to overcome above-mentioned hurdles [44-47].

On a preliminary study, the EW-CRDS technique has been combined with the thinlayer electrochemical cell, by inserting a Pt working electrode close to the silica prism surface, to study the change of solution $\mathrm{pH}$ [48], surface concentration of a redox species [49], and monitoring surface redox reactions spectroscopically [50]. These studies will be reviewed in section 1.6.3.

\subsection{Review of EW-CRDS Developments and Applications}

EW-CRDS is highly sensitive tool for studying changes at interfaces with sufficiently fast temporal resolution (with rapid pulse diode laser). EW-CRDS has been deployed using different geometries to study various processes at the solid/liquid interface. The modification of the TIR surface make the EW-CRDS excellent tool to investigate a wide range of applications such as bio-molecular interactions, biomaterials, biosensors, and electrochemical interaction at interfaces.

\subsubsection{Monitoring Molecular Adsorption with EW-CRDS}

The availability of suitable analytical tools that can follow, in real-time, the molecular surface-immobilization process at sub-monolayer levels is crucial to evaluate and optimize the outcome of any adsorption strategy.

One of the first studies of molecular adsorption was the use of EW-CRDS to study

the adsorption kinetics of $I_{2}$ at the silica surface of Pellin-Broca prism. Successive 
adsorption and desorption at sub-monolayer level were observed, and it was demonstrated that the $\mathrm{I}_{2}$ adsorption followed Langmuir adsorption isotherm [38]. In a follow-up study, the molecular orientation of $\mathrm{I}_{2}$ molecules on the silica surface was investigated using polarization-sensitive EW-CRDS [51]. The molecular orientation of Methylene Blue dye at the air/fused-silica interface was similarly investigated [52].

A number of EW-CRDS studies have considered molecular adsorption at the silica/water interface by manipulating experimental conditions. For example, the adsorption of Cresyl Violet dye has been studied as a function of the $\mathrm{pH}$ and the ionic strength of the solution [53]. Additionally the average molecular orientation angle of Crystal Violet at the $\mathrm{CH}_{3} \mathrm{CN} /$ silica interface has been studied as a function of polarization and surface concentration[54]. The pH-dependence of the adsorption of a Nile Blue dye derivative at the silica/water interface has also been investigated [55].

Many studies has been developed to investigate the effect of different interfaces on the adsorption process of molecular species and obtain information about interfacial electrostatic interactions. Where the EW-CRDS surface can be functionalized by supporting monolayer or multilayer assemblies such as polymers. These study includes the investigation of the effect of polyelectrolytes such as poly-L-lysine (PLL) and poly-Lglutamic acid (PGA) on the adsorption of tris(2,2'-bipyridine)ruthenium(II), $\mathrm{Ru}(\mathrm{bpy}) 3^{2+}$, as a function $\mathrm{Ru}(\mathrm{bpy}) 3^{2+}$ concentration, solution $\mathrm{pH}$, and ionic strength [48]. In addition, the adsorption of charged and uncharged dyes at different interfaces such as silica/acetonitrile $\left(\mathrm{CH}_{3} \mathrm{CN}\right)$ and under flow-injection conditions has been reported [14, 56]. In most recent work, the adsorption behavior of rhodamine $\mathrm{B}$ onto different interfaces, namely 
silica/methanol, silica/water and chlorotrimethylsilane-functionalized silica/methanol interfaces has been investigated. For each interface, the adsorption and de-sorption constants were optically measured [57].

\subsubsection{Biological Systems Interfaces Studies}

Studying and controlling the adsorption of biomolecules at solid/liquid interfaces plays a vital role in developing novel biosensors and tissue materials, manufacturing food and new pharmaceutical drugs, and efficiently performing enzymatic catalysis among many other applications [58-62].

There have been many investigations to study the adsorption processes of biological molecules on different surface assemblies. For example, the effectiveness of positively charged polymer coatings to minimize the adsorption of Cytochrome C (Cyt-C) compared to bare silica was investigated. They used a $70^{\circ}$ dove-tail prism with light in a normal incidence configuration at the entrance and exit surface of the prism to reach a photon lifetime of about 25 - $40 \mathrm{~ns}$ for the empty resonant cavity (in the absence of any analytical chromophores). The prism surface was modified using a single layer of polybrene and a triple layer of polybrene, dextran sulfate and polybrene in order to study possible inhibitors for protein adsorption. It was found that these coatings effectively suppress the irreversible adsorption of Cyt-C [20] . The adsorption behavior of Cyt-C protein on different selfassembled monolayers has been investigated, namely: bare silica, $\mathrm{C}_{18}$-coated, and $\mathrm{NH}_{2}$ coated. This study revealed that the adsorption of Cyt-C was irreversible on all modified surfaces, and the highest affinity towards the bare silica surface followed by the $\mathrm{NH}_{2}$ coated and the $\mathrm{C}_{18}$-coated [40]. 
Wang et al. demonstrated the sensitivity of EW-CRDS to femtomole quantities of an antigen, interacting with a surface-immobilized antibody: the antibody was immobilized at an (amino-propyl) functionalized prism surface and the ring-down time was monitored during addition of the dye-labelled antigen [63].

Everest et al. studied the polarization-dependent adsorption process of hemoglobin protein on fused silica surface using a Dove prism. By using a folded cavity configuration (normal incidence), they were able to measure very slight changes in molecular orientation [64]. Martin et al. demonstrated the potential of EW-CRDS as a medical tool in Hemoglobinuria diagnosis by studying Hemoglobin adsorption, from urine samples, to silica [65].

Very recently, Zhang et al. studied the kinetics of the adsorption of 5,10,15,20tetrakis(4-N-methylpyridiniumyl) porphyrin (TMPyP) from $\mathrm{pH} 7.4$ phosphate buffer solution (PBS) to a silica surface [66]. The TMPyP was delivered by a jet nozzle in order to study relatively fast adsorption kinetics, which were analysed using complementary finite-element modelling. After the surface had been functionalized with TMPyP, it was shown that calf thymus DNA efficiently desorbed the TMPyP from the surface, most likely by intercalation of TMPyP between the DNA base pairs. The kinetics of the process were deduced. These initial exemplar studies provide a platform and impetus for the further application of EW-CRDS to study processes related to physical biology. 


\subsubsection{Monitoring Electrochemical Processes with EW-CRDS}

The EW-CRDS combined with a thin layer electrochemical cell becomes a spectroelectrochemical technique. On a preliminary study, the EW-CRDS tool with the thin-layer electrochemical cell was used for electrochemical studies, ferricyanide, $\left[\mathrm{Fe}(\mathrm{CN})_{6}\right]^{3-}$, was electrochemically generated from ferrocyanide, $[\mathrm{Fe}(\mathrm{CN}) 6]^{4-}$, at the working electrode [49]. At the chosen light wavelength $(417 \mathrm{~nm})$, the oxidized species have

(a)

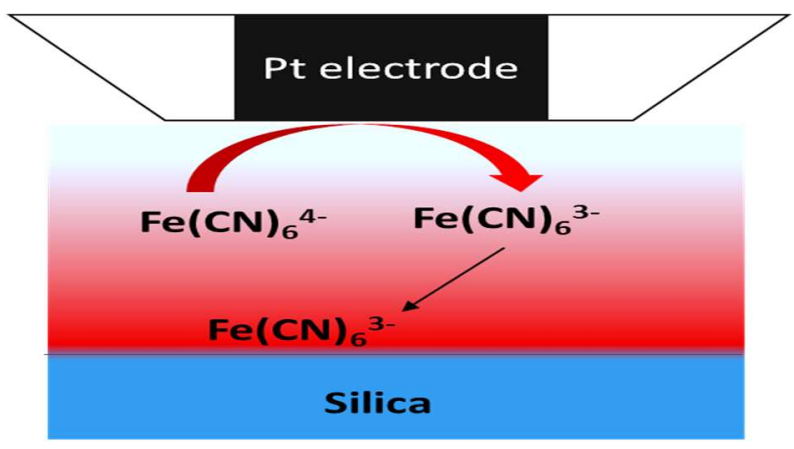

(b)

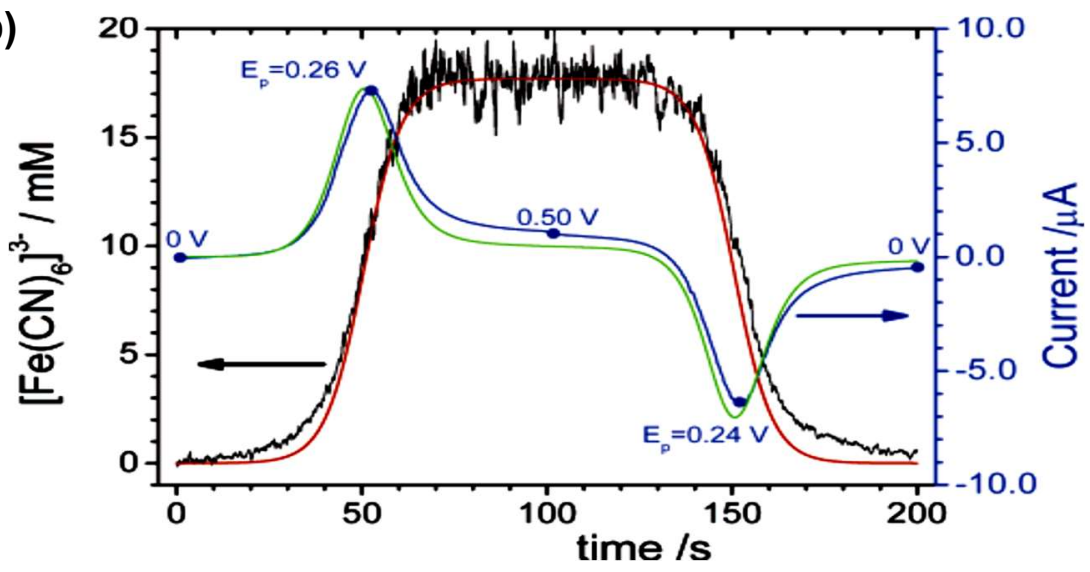

Figure 1-4: (a) Schematic representation of the thin-layer cell for EW-CRDS showing the generation of $\mathrm{Fe}(\mathrm{CN})_{6}{ }^{3-}$ from $\mathrm{Fe}(\mathrm{CN})_{6}{ }^{4-}$. (b) Ferricyanide concentration and the generated current, as a function of time, recorded during the $\mathrm{CV}$ scan $(5 \mathrm{mV} / \mathrm{s})$. The red (concentration) and green (CV) solid lines are simulations based on finite element modelling of a reversible process [30]. 
higher absorbance. In this work, cyclic voltammetry (CV) scans under synchronous optical readout were performed to measure the concentration of the electrogenerated species within the evanescent field, as shown in Figure 1-4(a). Figure1-4(b) shows the change in $\left[\mathrm{Fe}(\mathrm{CN})_{6}\right]^{3-}$ concentration at the prism/electrolyte interface and the current signal for oxidation and reduction at the electrode, as a function of time, during the $\mathrm{CV}$ potential scan, where the electrode-surface distance was $25 \pm 1 \mu \mathrm{m}$. The CV scan was set with an electric potential ranging from $0 \mathrm{~V}$ to $+0.5 \mathrm{~V}$. As the potential increases, the $\left[\mathrm{Fe}(\mathrm{CN})_{6}\right]^{3-}$ concentration increases (the absorbance increases), and reaches a maximum value (at oxidization peak, $E p=0.26 \mathrm{~V}$ ), which corresponds to the initial bulk concentration of $\left.\left[\mathrm{Fe}(\mathrm{CN})_{6}\right]^{4-}\right)$.When the applied potential is reversed, this causes $\left[\mathrm{Fe}(\mathrm{CN})_{6}\right]^{3-}$ to reduce back to $\left[\mathrm{Fe}(\mathrm{CN})_{6}\right]^{4-}$ as a result the absorbance and hence the concentration-decreases again.

In later studies, researchers studied the development of thin-layer electrochemical cell EW-CRDS to study the electrochemical properties of adsorbed silver nanoparticles (Ag NPs) on PLL-modified silica [32].The Ag NPs were electrochemically dissolved by using the diffusion-limited $\mathrm{IrC}_{6}{ }^{2-}$ redox mediator. As shown in Figure 1-5(a), $\operatorname{Ir}(\mathrm{IV})$ was electrogenerated from $\operatorname{Ir}(\mathrm{III})$ at a Pt electrode, by stepping the potential for $10 \mathrm{~s}$. The transient in optical absorbance during Ag NPs dissolving is shown in Figure 1-5(b). We notice after a short lag time (approximately $5 \mathrm{~s}$ ), due to the diffusion of $\operatorname{Ir}(\mathrm{IV})$ from the electrode to the prism surface, the optical absorbance decreases with time, which indicating dissolving and shrinkage of the Ag NPs [67]. 
In a subsequent work, EW-CRDS was used to change the solution $\mathrm{pH}$ in a controlled way via the potential-pulsed chronoamperometric oxidation of water. By stepping the electrode potential to very positive values, $\mathrm{H}^{+}$ions were generated in a quantitative manner

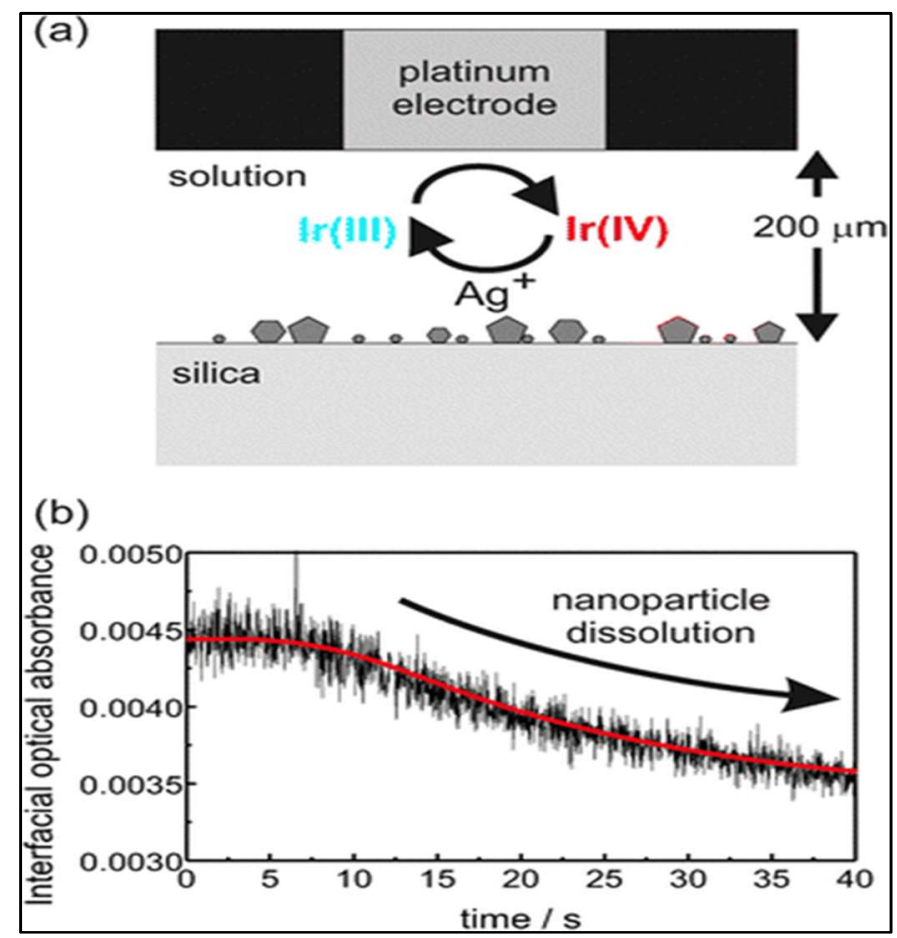

Figure 1-5: (a) Scheme of the thin layer electrochemical cell to dissolve the Ag NPs by electrochemically generation of $\operatorname{Ir}(\mathrm{IV})$ from $\operatorname{Ir}(\mathrm{III})$ by stepping the potential for $10 \mathrm{~s}$. (b) Absorbance transients (black line) and theoretical fit (red line) for a typical dissolution experiment [55].

to protonate the PGA layer, leading to the release of $\mathrm{Ru}(\mathrm{bpy}) 3^{2+}$ from the interface. Because the electrochemical generation of $\mathrm{H}^{+}$was well defined, it was possible to titrate the PGA film quantitatively by simply changing the potential step time or the electrode/prism separation and thus generate different $\mathrm{pH}$ values at the interface [48]. 
In the most recent study, the EW-CRDS tool with the thin-layer electrochemical cell was applied to investigate the adsorption process of $\mathrm{Cyt}-\mathrm{C}$ protein at a silica/water interface [50]. In this last report, as Figure 1-6 (a) shows, a chronoamperometry technique was applied to provide a soluble redox mediator $\left(\mathrm{Fe}(\mathrm{EDTA})^{2-}\right)$, which after diffusion, could chemically control the redox process of an adsorbed layer of Cyt-C species. The potential

(a)
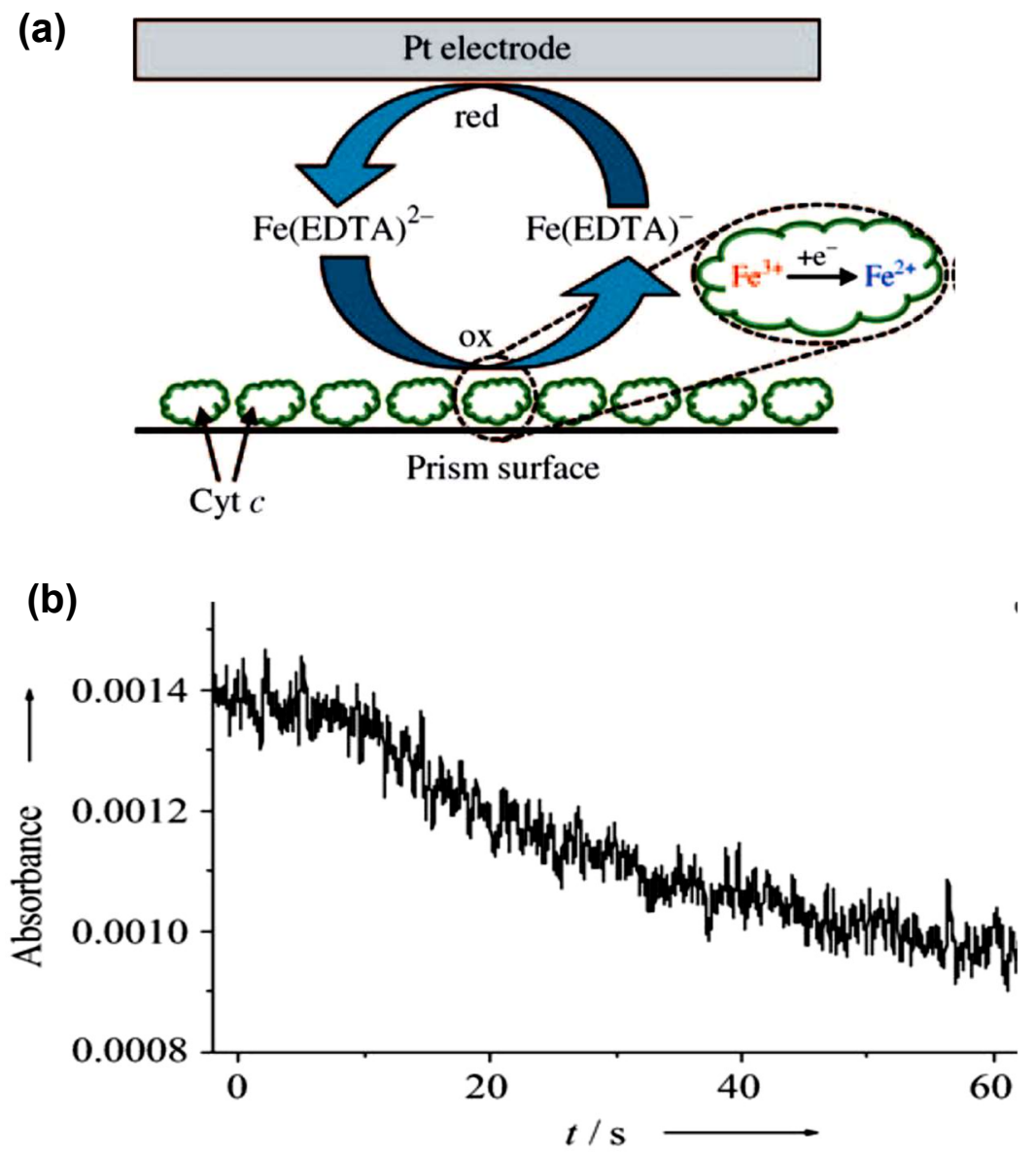

Figure 1-6: (a) Schematic representation of the thin-layer cell was used to measure the electron transfer constant during the reduction of immobilized Cyt-C by a redox mediator. (b) The optical signal response of EW-CRDS device at $400 \mathrm{~nm}$, as a function of time during a potential step [38]. 
stepped from $0 \mathrm{~V}$ to $-0.5 \mathrm{~V}$ for $60 \mathrm{~s}$, then returned to open potential Simultaneously, the optical signal transient was recorded $400 \mathrm{~nm}$, as shown in Figure 1-6(b). By modelling the thin-layer-cell arrangement, they were able to obtain the electron transfer (ET) rate between the redox mediator and immobilized Cyt-C.

Although, these studies highlight the EW-CRDS as a powerful tool to monitor the concentration changes during electrochemical processes by measuring the optical signal response, which is important for understanding the mechanisms of kinetics reaction. However, in all of these studies, the surface under optical interrogation by the EW-CRDS platform was not electrically active, so the redox process probed by the EW-CRDS signal was always limited by the mass diffusion of the redox agents. This can impose limitations for many electrochemical studies as for instance in investigations of the time rates of charge transfer in particular redox events. 


\subsection{Outline of the Dissertation}

In Chapter 2, the design, optimization, and performance of condensed phase CRDS experiments are described. Many optimizations and designs have been developed to achieve high sensitive measurements. To characterize the different CRDS geometries, the minimum detectible absorption coefficient was determined. Chapter 3 describes the application of an electro-active interface for the EW-CRDS tool. The coating of the EWCRDS surface with transparent and conductive layer of optimized ultra-thin indium tin oxide (ITO) film enables spectroelectrochemical investigations of redox events at solid/liquid interfaces by using the evanescent field of a cavity-confined light pulse. The effects of an applied electric potential to the adsorption behavior of the redox protein onto different interfaces factionalized on the surface of EW-CRDS platform surface are investigated; the time dependent adsorption kinetics, surface density of a redox-active protein probe at different interfaces are measured by EW-CRDS platform. The total surface coverage and active surface coverage are calculated based on the spectroscopic measurement during the $\mathrm{CV}$ potential scan. In chapter 4 , the detection, identification, and quantification of a targeted virus antigen using a sandwich bioassay on the electro-active EW-CRDS device is demonstrated. Chapter 5 describes the preliminary demonstration of the liquid-phase CRDS for the detection and quantification of trace amount of nitrite ions in drinking water. The potential of the CRDS technique to measure minute absorption loss with a precision of better than $1 \mathrm{ppm}$ in the ITO ultra-thin film during UV treatment will be addressed in Chapter 6. Some conclusions regarding the research described in this dissertation, as well as outlooks for possible applications of condensed phase CRDS platforms will be presented in Chapter 7. 


\section{CHAPTER 2}

\section{DESCRIPTION, OPTIMIZATION, AND PERFORMANCE OF A CONDENSED PHASE CRDS EXPERIMENTS}

\subsection{Introduction}

Condensed-phase CRDS enables interfacial absorption measurements at a very high sensitivity. Compared with gas-phase, the implantation of CRDS in condensed matter introduces optical loss due to any absorptive, scattering, and reflection losses within any optical components or solvent inside the cavity. Leading to a range of novel designs of the optical cavity to increase the sensitivity of the technique. This chapter describes the different geometries of the CRD spectrometer used throughout this work, the CRDS signal acquisition system, as well as the ring-down time measurements and the electrochemistry data acquisition. However, most of the experimental details are given in the relevant research chapters, since they provide a better context alongside the experimental techniques.

The optical loss throughout each EW-CRDS configuration was measured to find out the most sensitive cavity arrangement with the high quality factor. For each EW-CRDS geometry used, the optical loss was measured experimentally and compared with the theoretical calculations. To assess the performance of each configuration for sensitive 
optical absorbance measurement, the minimum detectable absorption coefficient was also measured.

\subsection{Cavity Configurations in Condensed Phase CRDS}

Optical cavities can be implemented to condensed phase with different designs [25, 42], depending on the physical condition of the sample under study, where the optical cavity can be used to monitor the absorbance either at solid/liquid or solid/air interfaces. The following sections describe the condensed phase CRDS configurations used throughout this work.

\subsubsection{Confocal Linear (on-axis) Cavity}

A leaner confocal cavity arrangement has been previously used by many groups $[14$, 53]. As shown in Figure 2-1, the highly reflective mirrors were placed opposite one another to form a linear cavity. A standard right-angle and antireflection-coated (PS908L-A, $\mathrm{R} \cong 0.5 \%$ at normal incidence, Thorlabs) prism was used and placed, at the midway point

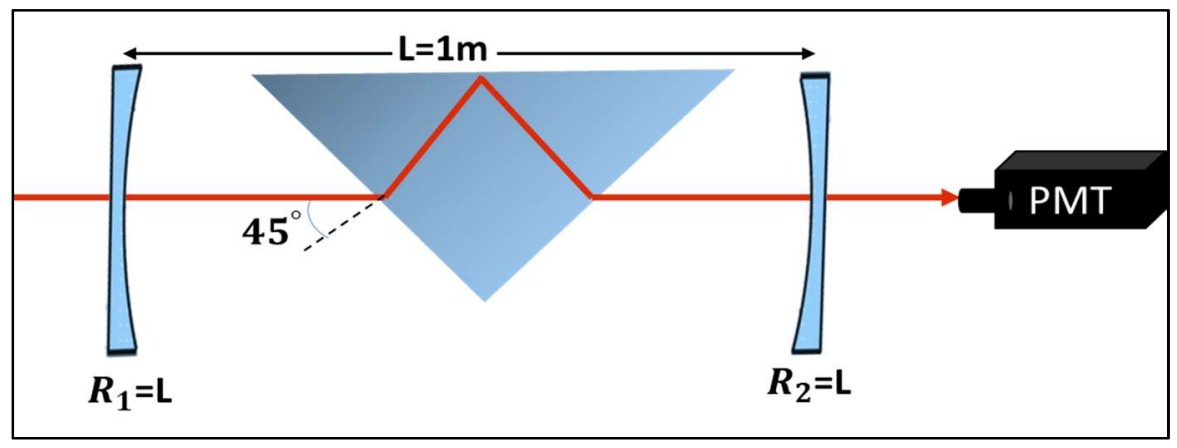

Figure 2-1: Schematic representation of the confocal linear cavity employing coated right-angle prism. In a confocal geometry, the radii of curvature of the two mirrors $\left(\mathrm{R}_{1}\right.$ and $\mathrm{R}_{2}$ ) should equal the cavity length (L). 
between the mirrors, in the way that a laser beam enters and exits the prism with an angle of incidence of $45^{\circ}$. The disadvantage of this configuration is the surface reflective losses from the prism sides, which limit the sensitivity. This configuration is described in Chapter 3.

\subsubsection{Brewster's Angle Configuration (off-axis) Cavity}

Many studies of EW-CRDS have been carried out with expensive, custom-made prisms $[20,39,64]$. In this work, a Brewster's angle configuration was deployed to minimize reflection losses, as reported in previous studies $[38,63]$. In this configuration, the laser beam enters the cavity through the back of highly reflective mirror and hits the surface of the uncoated prism close to the edge at Brewster's angle, as shown in Figure 22. This approach has two disadvantages, considering that the Brewster's angle is slightly wavelength dependent, and the reflectivity goes to zero at the Brewster's angle for only ppolarized light. However, this geometry is desirable to have low loss into the optical cavity; it allows incorporation of a low-cost standard prism within a high finesse optical cavity and can be used over wide range of wavelengths. This geometry will be employed in Chapter 3 and 4.

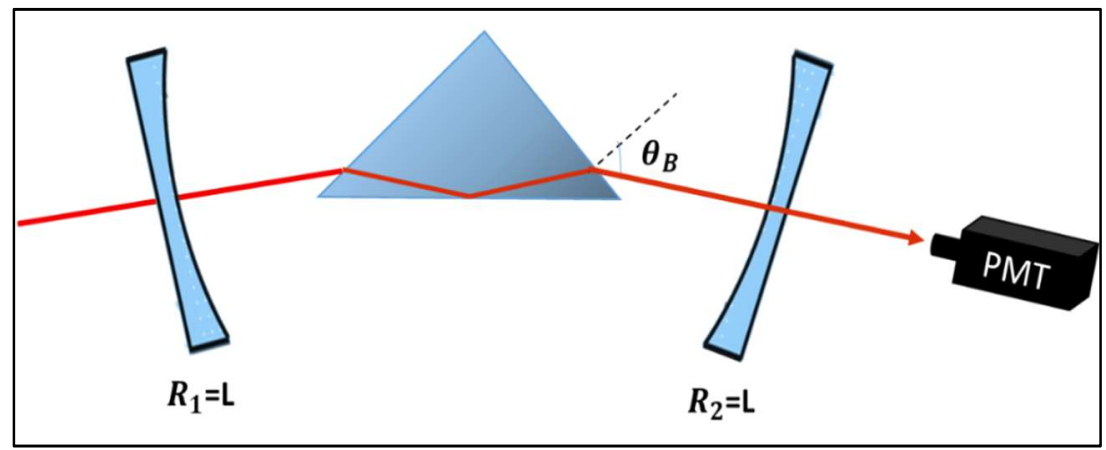

Figure 2-2: Schematic representation of a Brewster's angle cavity employing uncoated rightangle prism. The laser beam hits the prism surface close to the edge at Brewster's angle. 


\subsubsection{Thin film on Brewster's Angle Substrates Configuration}

The way to extend CRDS from gas state to solid state is to put optically transparent thin film on glass substrate inside the optical cavity. As shown in Figure 2-3, the laser beam hits the glass slide at Brewster's angle of glass. Application of this configuration is subject to many constraints: The glass substrate should have high quality and must be highly transparent in order to minimize absorption scattering. In addition, the refractive index of the substrate must be as close as possible to the index of refraction of the thin film sample. An index mismatch between the sample and the substrate results in background reflective losses, reducing the measurement sensitivity. However, when the sample thickness is small compared with the light source wavelength, the reflections from the air-film interface and the film-substrate interface interfere destructively, significantly reducing the reflective losses [37]. All these issues will be addressed in Chapter 6 .

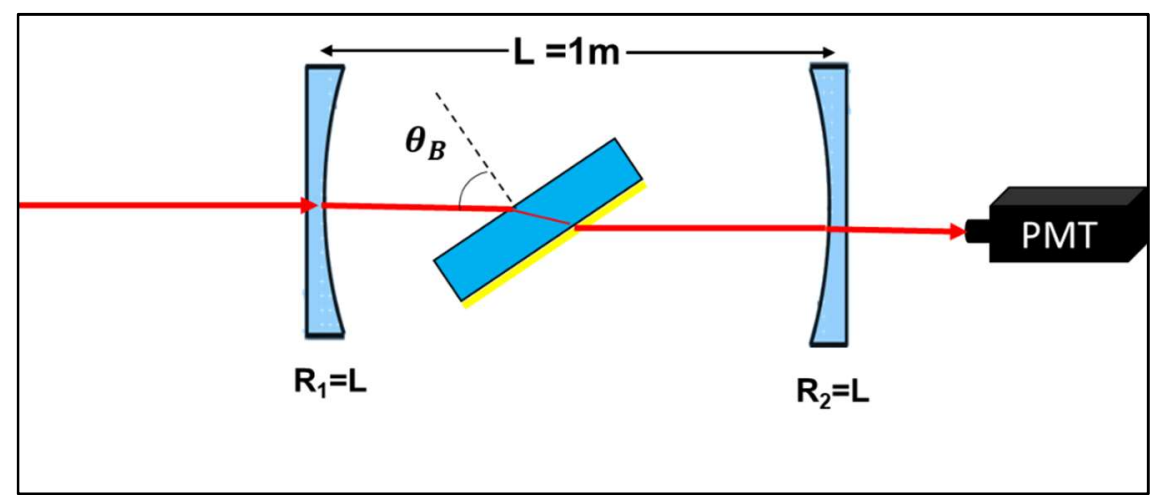

Figure 2-3: Schematic representation of a thin film CRDS. The glass substrate is placed inside the optical cavity in such way that the laser beam enters and leaves the substrate at Brewster's angle of glass. 


\subsubsection{Leaner Cavity with Normal Incidence Cuvette}

Liquid phase CRDS can be constructed by inserting a quartz cuvette at normal incidence inside a linear cavity configuration, as shown in Figure 2-4. This method has the advantage that light reflected off each surface of the cuvette is captured along the cavity axis and, therefore, does not contribute to the loss per pass through the cavity. Which offers higher sensitivity than the conventional UV-visible spectroscopy in molecular detection in bulk solution as previously reported $[68,69]$. This configuration will be employed in chapter 5 .

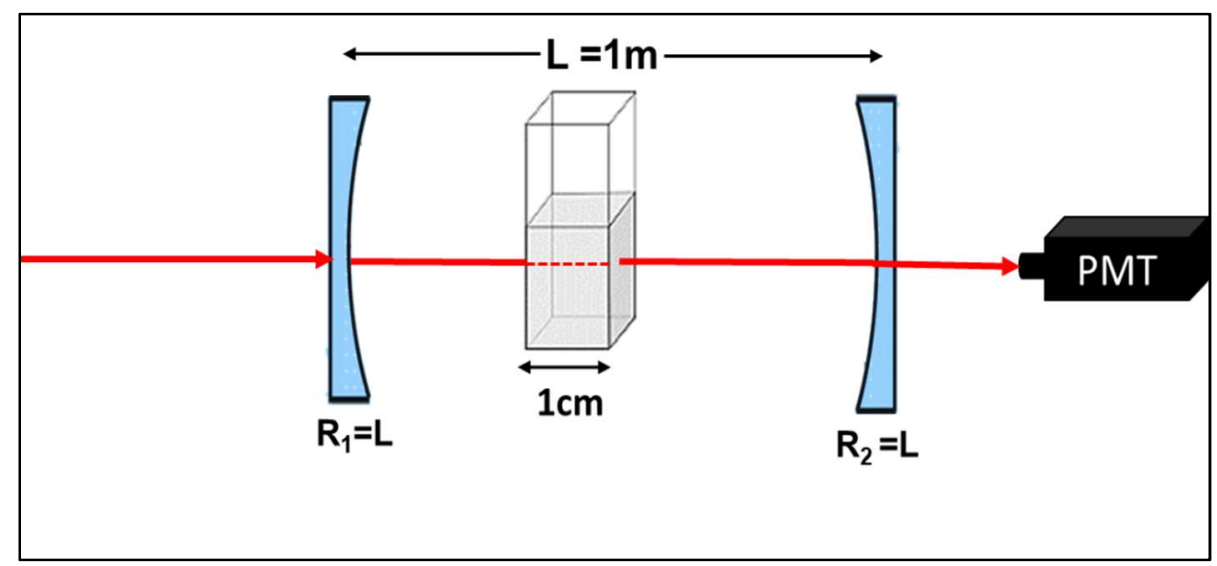

Figure 2-4: Schematic representation of liquid phase CRDS, the light enters and leaves the linear cavity perpendicular to the planar sides of the quartz cuvette.

\subsection{Data Acquisition and Ring-Down Time Measurements}

\subsubsection{Data Acquisition of EW-CRDS Experiment}

The oscilloscope acquires the cavity ring-down signal data from a PMT in equivalent real-time. The oscilloscope was triggered by a pulsed Nd:YAG laser. Then, the data was 
transferred into a computer via a GPIB to Ethernet interface connection, the averaging of ring-down time (typically between $8-64$ laser pulses) is recommended to eliminate the shot-to-shot noise from the laser energy fluctuations [70]. The averaged ring down traces were used to calculate the ring-down time $\tau$, using least-squares fitting routine via a custom- LabView program to ensure data synchronization.

In order to capture each laser beam round trip (approximately every $6.7 \mathrm{~ns}$ for the cavity of length $1 \mathrm{~m}$ ) in the optical cavity, the oscilloscope needs to acquire the signal data at a minimum data acquisition rate of $150 \mathrm{MHz}$. This is achieved, as the oscilloscope used for these experiments acquires data at $1 \mathrm{GHz}$. An example of the typical single shot, ringdown traces is shown in Figure 2-5.

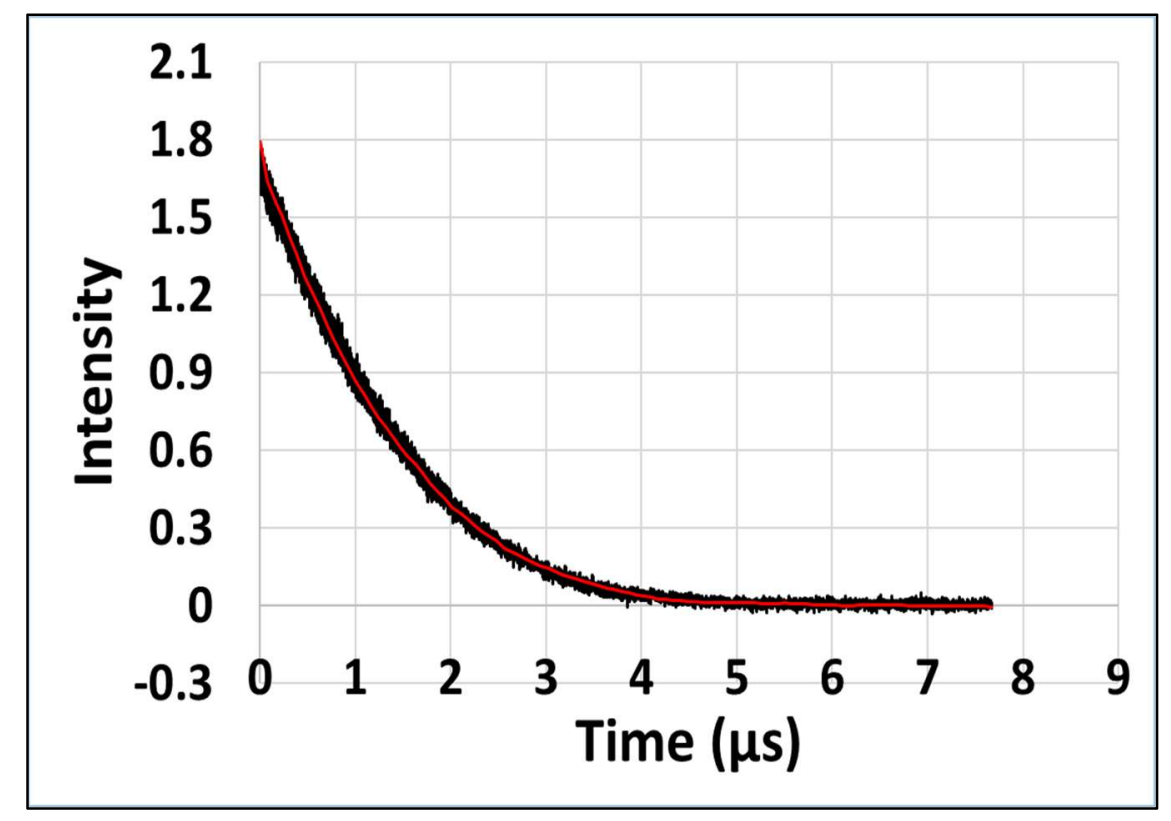

Figure 2-5: Experimental single-shot cavity-ring-down trace obtained with the Brewster's angle EW-CRDS setup. The red line is an exponential decay fit to the data. 
The LabView program is based on modules, which are called virtual instruments (VIs).These VIs consist of a front panel, which is designed to control data acquisition and averaging ring-down time from the oscilloscope (i.e. with switches, controls, graphs, etc.), extraction of the corresponding ring-down time, and a back panel which is called "block diagram," where the code of the program can be written and/or modified.

Figure 2.6 shows the screenshot of Labview VI front panel used for EW-CRDS data acquisition. This program consists of sub-VIs, which are used to set the oscilloscope parameters and plot the CRD signal as a function of time (Figure 2-6(a)). Another sub-VI is the exponential decay function fit of EW-CRD signal, while a different sub-VI is used to record and save the ring-down time as a function of time (Figure 2-6(c)).

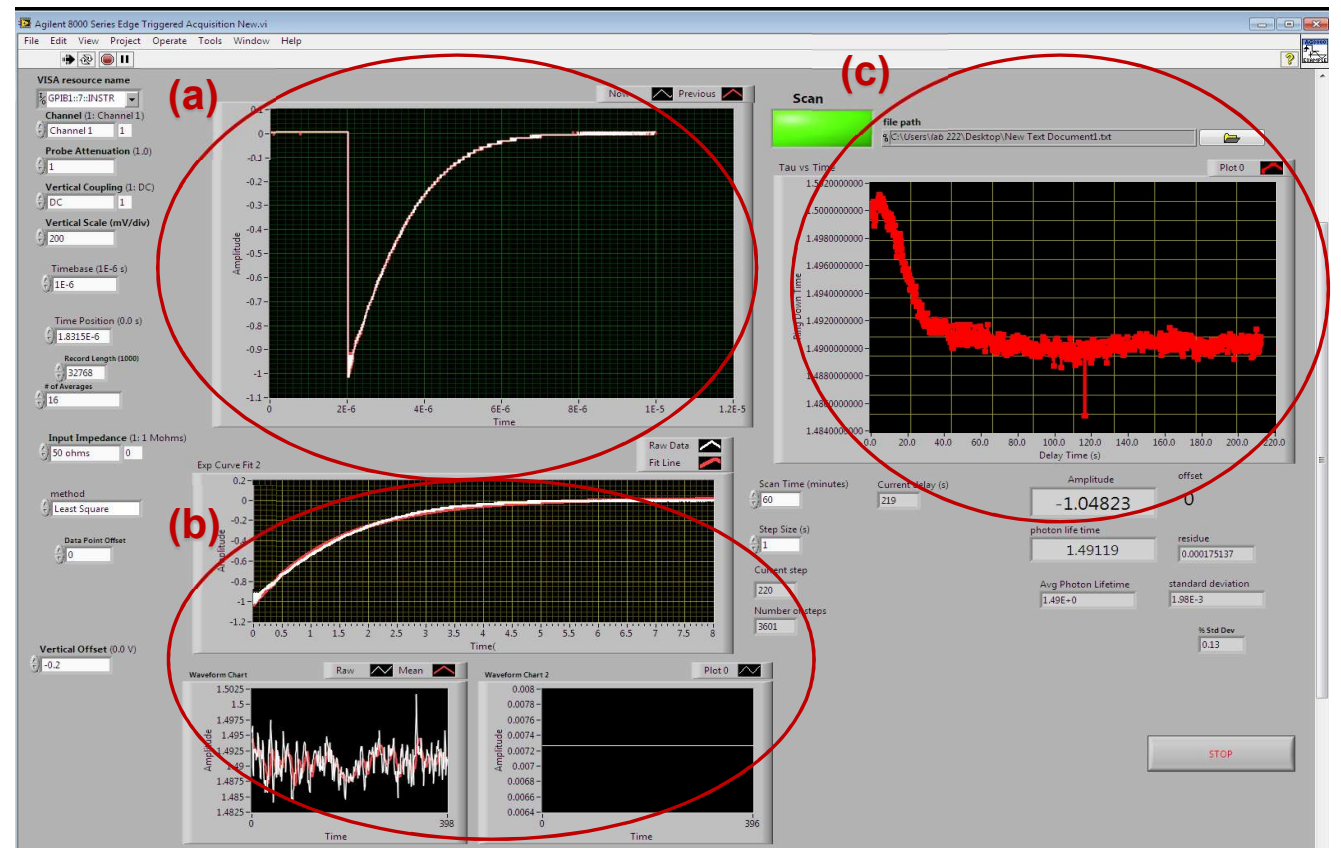

Figure 2-6: Screenshot of the Labview front panel used for EW-CRDS data acquisition. (a) sitting the oscilloscope parameters and plot of CRD signal as a function of time, (b) exponential decay function fit, and (c) sub-VI used to plot and save ring-down time as a function of time. 


\subsubsection{Electrochemistry Data Acquisition}

The Labview program is combined with an EW-CRDS program to ensure the synchronization of optical data of ring down time and the electrochemical data under $\mathrm{CV}$ scan. Figure 2-8 shows a screenshot of the front panel of the program used for EW-CRDS and CV data acquisition. The Labview VI was design to extract the EW-CRDS ring-down time and the electrochemical measurements simultaneously.

A commercial potentiostat was used to control the potential at the working electrode and was connected to the computer via USB. In the first step, the program reads and sets the parameters, then once the start button is pushed, a separate sub-VI is used to extract the electrochemical data. Simultaneously, the EW-CRDS ring-down times are recorded every $0.8 \mathrm{~s}$ (the time of 8 laser pulses average) to ensure the optical and electrical synchronization. The generated ring-down time and voltage are then save by the computer (block diagram of the EW-CRDS data acquisition can be found in appendix 1).

As can be seen in Figure 2-7, the program front panel has two taps: the parameters tap is used to set the oscilloscope and CV parameters (Figure 2-7 (a)). The main tap shows the Start and Stop data acquisition buttons, and the plots for data display, location and function as follows: Top right, CRDS signal as a function of time; bottom middle, exponential decay function fit; top left, CV scan plot of the electric current as a function of applied voltage; bottom left, applied potential for CV experiments; bottom right, the extracted potential data. 

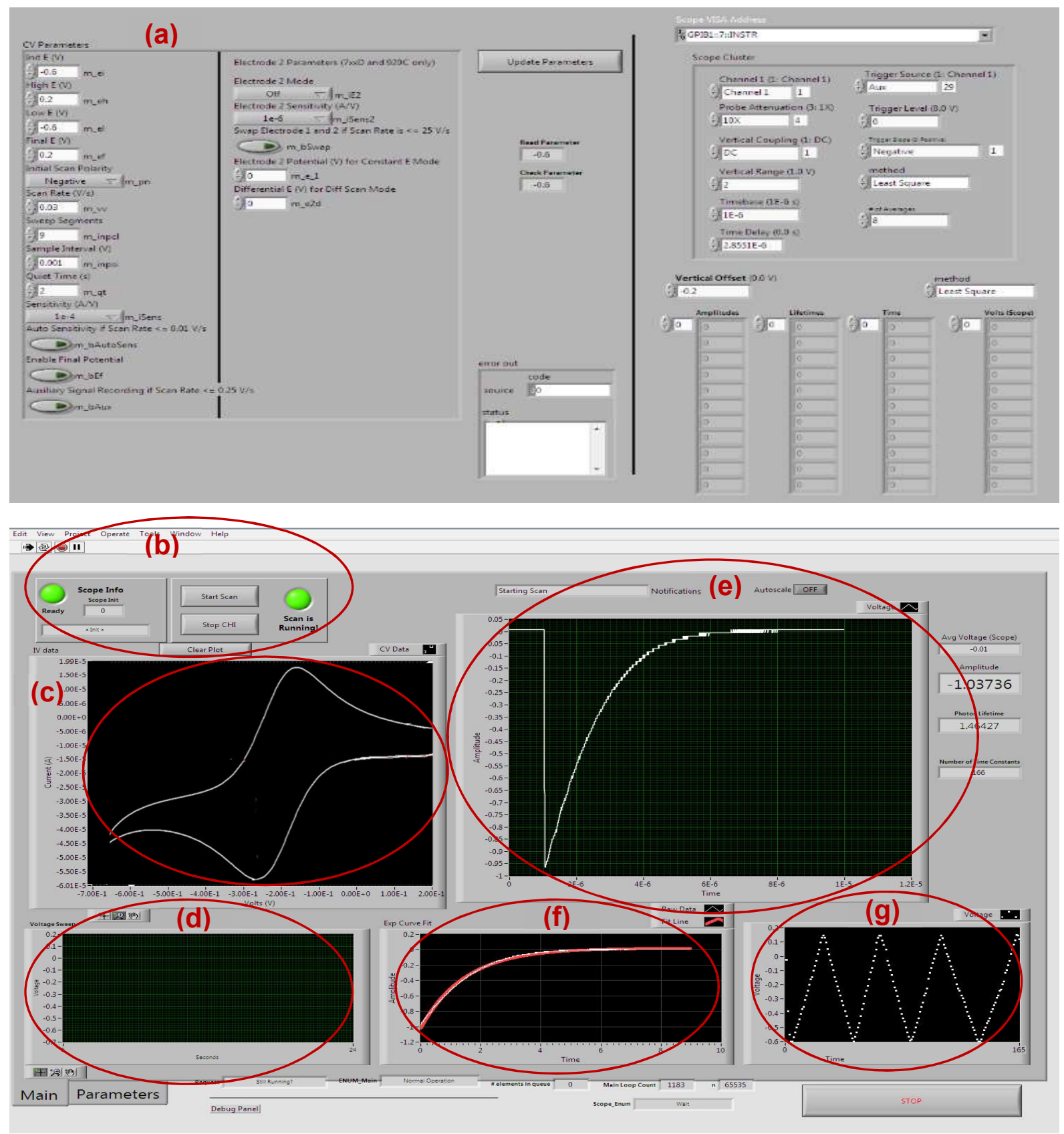

Figure 2-7: Screenshot of the front panel of the LabVIEW program used for spectroelectrochemical experiment data acquisition. (a) Settings the oscilloscope and CV experiments parameters tap, (b) Start switch for performing an electrochemical experiment (CV) and time stamps for data synchronization, (c) plot of the electric current as a function of applied potential in CV scan, (d) plot of applied voltage as a function of time, (e) EW-CRDS signal as a function of time, (f) exponential decay fit of EW-CRDS signal, (g) extracted electric potential values. 


\subsection{Performance of CRDS Apparatus}

\subsubsection{Laser Performance}

A second harmonic of Nd:YAG (532 nm, 4-6 ns) laser used to obtain the CRDS signal was generated by frequency-doubling non-linear optics. For a stable optical resonator, it is critical that the lasers used to excite the cavity are performing optimally. The performance of any laser light can be measured by three main properties: pulse-topulse energy fluctuations, average pulse energy, and pulse-to-pulse timing jitter (i.e., the fluctuation in the time delay between the opening of the $\mathrm{Q}$ switch and generating the pulse, which results in some deviations of the temporal pulse positions from those in a perfectly periodic pulse train). A low average pulse energy will cause the signal-to-noise ratio (SNR) to decrease, while timing jitter or very large energy fluctuations will reduce the quality ring-down traces due to individual ring-downs exceeding the scale of the oscilloscope board [19, 71]. Table 2.1 summarizes a typical Nd-YAG laser performance and specifications for different operating wavelengths of light.

Table 2-1: Typical Surelite Nd-YAG laser (SL II-10) performance and specifications, for different operating wavelengths [72].

\begin{tabular}{|c|c|c|c|c|c|c|c|}
\hline $\begin{array}{l}\text { Wavelength } \\
(\mathrm{nm})\end{array}$ & $\begin{array}{l}\text { Repetition } \\
\text { Rate }(\mathrm{Hz})\end{array}$ & $\begin{array}{l}\text { Pulse- } \\
\text { width } \\
\text { (ns) }\end{array}$ & $\begin{array}{l}\text { Pulse } \\
\text { Energy } \\
(\mathrm{mJ})\end{array}$ & $\begin{array}{l}\text { Jitter } \\
( \pm \mathrm{ns})\end{array}$ & $\begin{array}{c}\text { Energy } \\
\text { fluctuation } \\
( \pm \%)\end{array}$ & $\begin{array}{c}\text { Beam } \\
\text { Diameter } \\
(\mathrm{mm})\end{array}$ & Polarization \\
\hline 1064 & \multirow{4}{*}{10} & $4-7$ & 650 & \multirow{4}{*}{0.5} & 2.5 & \multirow{4}{*}{7} & $\mathrm{H}$ \\
\hline 532 & & $4-6$ & 300 & & 3.5 & & $\mathrm{~V}$ \\
\hline 355 & & $4-6$ & $100 / 1602$ & & 4.0 & & $\mathrm{H}$ \\
\hline 266 & & $4-6$ & 80 & & 7.0 & & $\mathrm{H}$ \\
\hline
\end{tabular}




\subsubsection{Photomultiplier tube detector Performance}

For improving the sensitivity of CRDS spectrometer, it is required that the SNR of the recorded ring-down signal be as large as possible. The sensitivity of the instrument can be greatly improved simply by collecting and fitting the data over a large number of ringdown lifetimes.

A photomultiplier tube (PMT) was used to measure the cavity output intensity. By choosing a detector appropriate for our laser power and ring-down mirrors, we can maximize our SNR. For example, CRDS needs a sensitive photodetectors with enough bandwidth to follow the exponential CRD signal with good time resolution; if the bandwidth of the photodetector is too small, then the recorded ring-down signal will be distorted [15]. We can estimate the expected peak ring-down voltage as a function of mirror reflectivity. By comparing this value to the noise specification of the detector, one can estimate the SNR. The incident optical power that reach the PMT can be estimated as [73]:

$P_{0}=\frac{E_{\text {cavty }}}{\tau_{0}}=\frac{(1-R) E_{\text {laser }}}{\left[\frac{L}{c(1-R)}\right]}=f_{\text {inc }} \frac{(1-R)^{2} c E_{\text {laser }}}{L}$

where $E_{\text {cavty }}$ is the laser energy stored in the optical cavity, $\tau_{0}$ is the empty cavity ringdown time, $L$ is the cavity length, $\mathrm{c}$ is the speed of light, $\mathrm{R}$ is the mirror reflectivity, $E_{\text {laser }}$ is the energy per pulse of the incident laser light, and $f_{\text {inc }}=0.04$ is the fraction of light entering the cavity that actually couples to cavity modes.

The output voltage of a photodetector as a function of the incident optical power is given by: 
$V_{a}=P_{0} R_{f} \Re=R_{f} \Re f_{\text {inc }} \frac{(1-R)^{2} c E_{\text {laser }}}{L}$

where $\mathfrak{R}$ is the photodetector's responsivity or anode radiant sensitivity (in $\mathrm{A} / \mathrm{W}$ ), $R_{f}$ is the photodetector's transimpedance amplification (in V/A). The photodetector noise level can be given by the Equation $[73,74]$ :

$V_{\text {noise }}=\Re \times R_{f} \times N E P \times \sqrt{B}$

Where $N E P$ is the noise equivalent power (in $\mathrm{W} \mathrm{Hz}^{-1 / 2}$ ), and $B$ is the detector bandwidth. Table 2-2 summarizes the specifications of the PMT used throughout this work.

Table 2-2: Summary of the PMT specifications [75].

\begin{tabular}{|c|c|c|c|c|c|c|}
\hline $\begin{array}{c}\text { Spectral } \\
\text { Response } \\
(\mathrm{nm})\end{array}$ & $\begin{array}{c}\text { Frequency } \\
\text { Bandwidth } \\
\text { (KHz) }\end{array}$ & $\begin{array}{c}\text { Time response } \\
\text { (Pulse Rise } \\
\text { Time) } \\
\text { (ns) }\end{array}$ & $\begin{array}{c}V_{\text {noise }} \\
(\mathrm{mV})\end{array}$ & $\Re$ & $R_{f}$ & $\begin{array}{c}\text { Dark } \\
\text { Current } \\
(\mathrm{A} / \mathrm{W})\end{array}$ \\
\hline $300-650$ & DC to 20 & 0.65 & 1.2 & $18 \times 10^{4}$ & 1 & $0.04-0.02$ \\
\hline
\end{tabular}

\subsubsection{CRDS Mirrors performance}

High-reflectance mirrors, with reflectance values ranging from $99.8 \%$ up to 99.999\%, are essential for the high performance of the CRD spectrometers. A high enough reflectivity of the mirrors allows more data to be collected before it is fallen below the detector's signal-to-noise, this results in long ring-down lifetime. It is important to know the mirror reflectivity as a function of wavelength to properly classify the spectrometer. In order to determine the reflectivity of the employed mirrors, the vacuum ring-down times can be converted to the mirror reflectivities using Equation 1-5. 


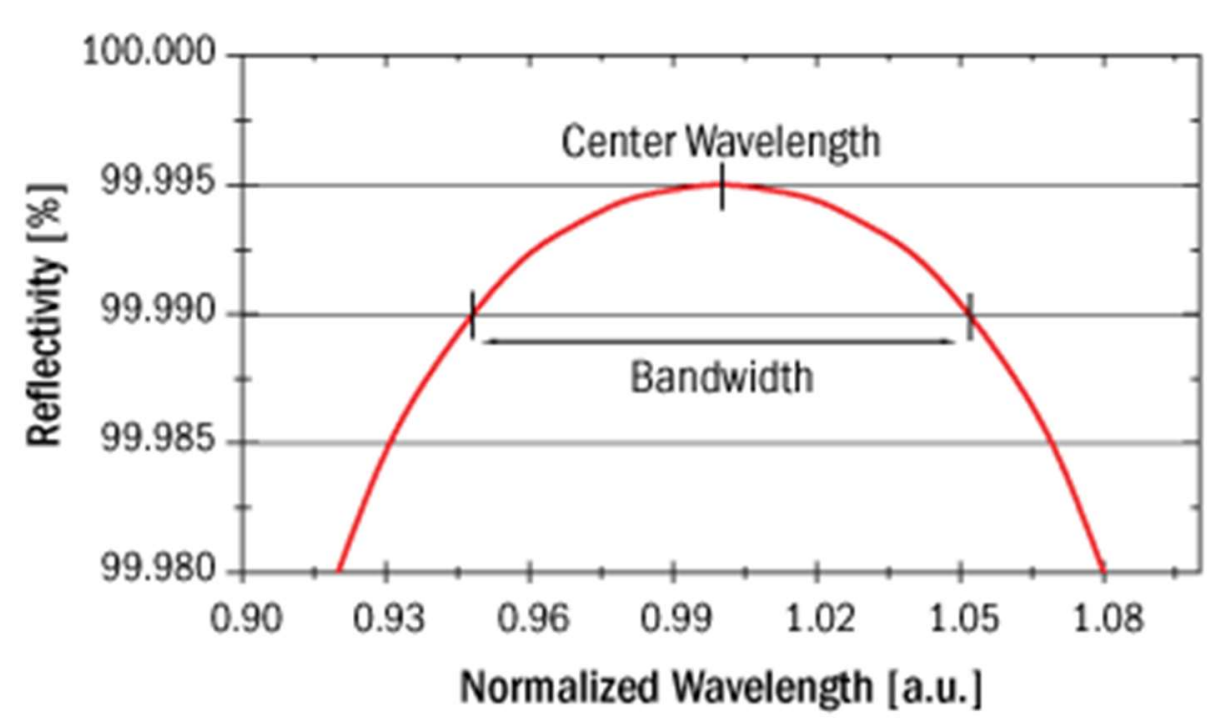

Figure 2-8: Measured mirror reflectivity vs. normalized wavelength for $532 \mathrm{~nm}$ CRDS optics dielectric mirrors forming the cavity [76].

Figure 2-6 shows the reflectivity curve of the mirror used throughout this work. As illustrated in the curve, the mirror reflectivity is largest at the center (at the peak wavelength, $532 \mathrm{~nm}$ ), and the ring-down time will be longest. The reflectivity decreases when we move further away from the peak wavelength, causing the ring-down time to decrease. The specifications of the CRDS mirrors used in this work are summarized in Table 2-3.

Table 2.3. High reflectivity CRDS mirror specifications [76].

\begin{tabular}{|c|c|c|c|c|c|}
\hline $\begin{array}{c}\text { Surface } \\
\text { Flatness }\end{array}$ & $\begin{array}{c}\text { Surface } \\
\text { Quality }\end{array}$ & $\begin{array}{c}\text { Peak } \\
\text { Reflectivity \% }\end{array}$ & $\begin{array}{c}\text { Center } \\
\text { Wavelength } \\
(\mathrm{nm})\end{array}$ & $\begin{array}{c}\text { Bandwidth } \\
(\mathrm{nm})\end{array}$ & $\begin{array}{c}\text { Damage } \\
\text { Threshold } \\
@\end{array}$ \\
$\begin{array}{c}\lambda / 10 \\
\left(\mathrm{~J} / \mathrm{cm}^{2}\right)\end{array}$ \\
$\begin{array}{c}20-10 \\
\text { 632nm }\end{array}$ & 99.995 & 532 & $497-542$ & 1 \\
\hline
\end{tabular}


For increasing the effectiveness of the spectrometer, the cavity mirrors must be kept clean over time, this can cause mirror surfaces and coatings damaged, leading to a decrease in reflectivity. To avoid this phenomenon, care must be taken to keep the mirrors as clean as possible to avoid the need for repeated cleaning.

There is an upper limit for the mirrors reflectivities; if the mirror reflectivity $(R)$ is too high, then not enough light will be able to enter the cavity, and the peak ring-down voltage $\left(V_{0}\right)$ will become too small to detect. It is therefore important to choose mirrors with a high enough reflectivity to create large ring-down times, while simultaneously transmitting enough light to be detected.

\subsection{Calculations of the Optical Loss in EW-CRDS Configurations}

Optical loss is a significant issue in the design and implementation of CRDS systems, since a low internal cavity loss is a requirement to achieve a lower detection limit. The ring-down times are limited by the optical loss inside the optical cavity. There are many sources for optical loss, including:

I) Misalignment of the cavity mirrors, leading to radiation inside the cavity not be confined during its path between the mirrors.

II) Absorption and scattering losses in optical elements. Since optical elements are not ideal, each interaction with optical elements inside the cavity will cause losses.

III) Diffraction losses. With the diffraction of a laser beam passing through a limiting aperture, sometimes increasing of the aperture size is not possible for reducing the 
diffraction, when such increase in aperture size would result in exciting undesired higher order transverse modes [77].

In this study, many attempts have been made to reduce cavity loss, including modifying the fabrication process to reduce scattering loss induced by sidewall roughness, employing high-confinement geometries with low optical loss, modifying the detection system, and so forth. This section shows the theoretical calculations of the optical loss in the EW-CRDS configurations (i.e. the confocal linear and the Brewster's angle configurations) used in order to find out the best configuration of the optical cavity with high quality factor. Furthermore, the optical loss was measured experimentally with EWCRDS platform and was compared with theoretical results.

For this purpose, the single-pass transmittance of light through the prism was measured experimentally by using two ways to ensure the accuracy of the measurements: Using a power meter and locking amplifier with signal modulation. Figure 2-9 shows the experimental setup. A continuous-wave (CW) laser (Shanghai Dream lasers Tech., SDL$532-150 \mathrm{~T})$ at the wavelength of $532 \mathrm{~nm}$ was used as the light source. The laser beam was directed through a half-wave plate and linear polarizer to establish linearly polarized light at transverse-magnetic (TM) polarization, for the on-axis cavity arrangement coated

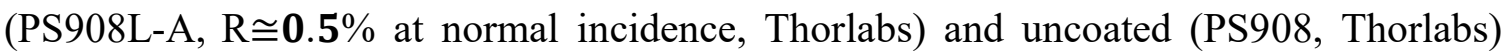
right-angle 20-mm N-BK7 were used. For Brewster's angle geometry, a standard uncoated right-angle 20-mm N-BK7 (PS908, Thorlabs) was used. The optical cavity was formed by two high-reflectivity concave mirrors $(\mathrm{R}=99.995 \%$ at $532 \mathrm{~nm}, 1-\mathrm{m}$ radii of curvature, CRD Optics, Inc.) mounted on adjustable mounts (CRD Optics, Inc.). 
In the first step, the average single- pass transmittance through the prism was measured, for each EW-CRDS configuration, using a power meter (Newport model 1930C) by taking the ratio of transmitted and incident light intensity (where, $T=I_{2} / I_{1}$ ).

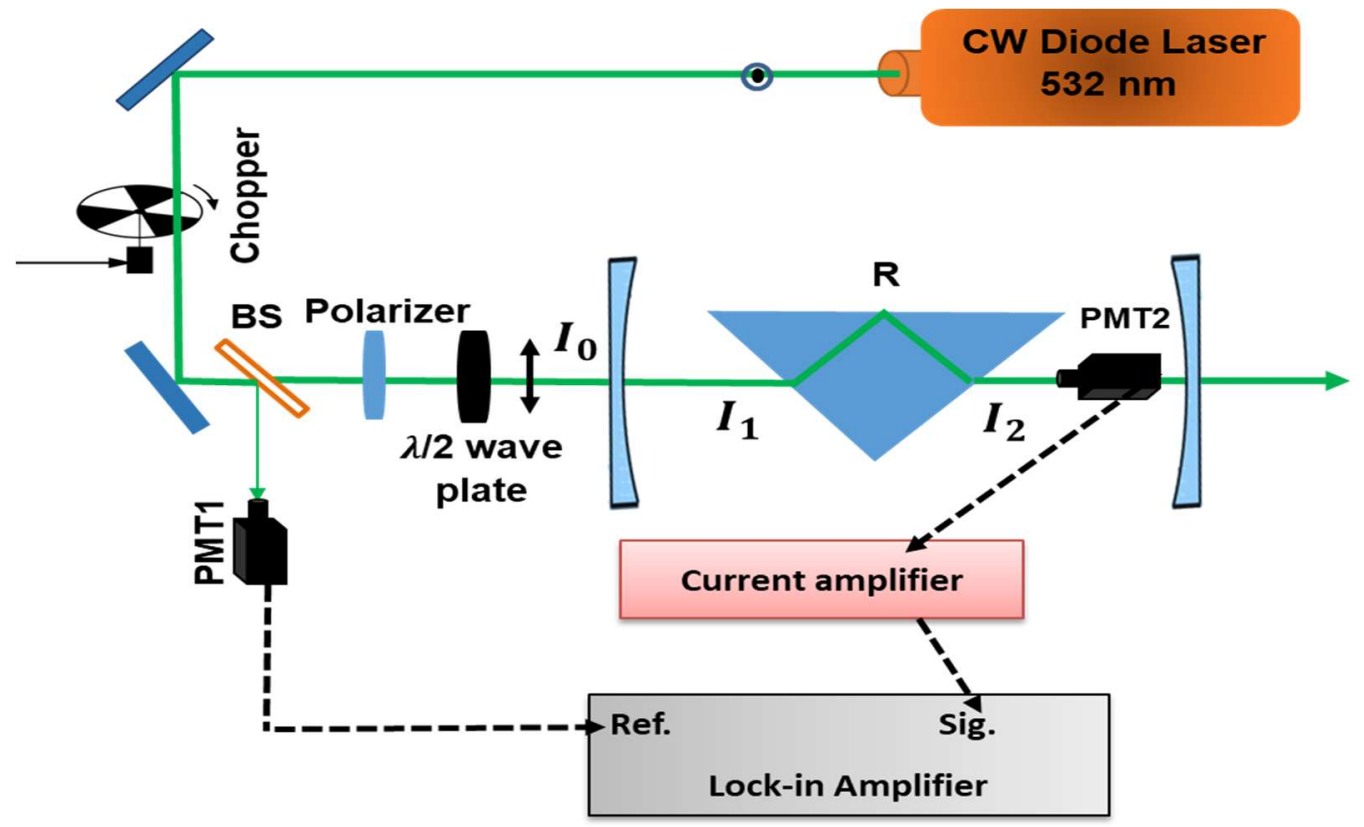

Figure 2-9: The experimental setup for a single-pass transmittance measurement through the prism. CW laser was modulated using a mechanical chopper, the laser polarization was chosen using a linear polarizer and half-wave plate. The reference signal intensity was detected by the photo detector (PMT1), while, the incident $\left(I_{I}\right)$ and the transmitted $\left(I_{2}\right)$ light intensities were measured by PMT2. The optical signal was amplified using current preamplifier and was fed to the lock-in amplifier.

Another way to measure precisely the transmittance is by modulating the intensity of laser beam using mechanical chopper (SR540, Stanford research system) at a frequency $200 \mathrm{~Hz}$ and a lock-in amplifier was used to improve the signal-to-noise ratio as shown in Figure 2-9. The light beam was split into two parts by using a beam splitter (BS); one was 
directed to the cavity and another one toward the Photomultiplier tube (PMT1) (PMM02, Thorlabs) to detect the input reference modulated signal. A similar PMT2 was used to measure the incident (from air to prism, $I_{1}$ ) and transmitted (from prism to air, $I_{2}$ ) modulated light intensities. The collected PMT2 signals were amplified using a current pre-amplifier before sending it to lock-in- amplifier (SR810, Stanford Research Systems).

The measured average single-pass transmittance was used to calculate the loss coefficient and a ring-down time as following:

The round trip optical loss factor $e^{-\alpha_{r}(2 L)}$ can be given by [78]:

$$
I_{0} R_{M_{1}} T_{P 1} R \quad R_{M_{2}} T_{P 2}=e^{-\alpha_{r}(2 L)}
$$

where $I_{0}$ is the intensity of the light enters the cavity, $R_{M_{1}}$ and $R_{M_{2}}$ are mirrors reflectance $\left(R_{M_{1}}=R_{M_{2}}\right), T_{\mathrm{p} 1}$ and $T_{\mathrm{p} 2}$ are a single pass transmittance from air to prism and from prism to air respectively, $\mathrm{R}$ is the total internal reflectance $(\mathrm{R} \cong 1), L$ is the cavity length, and $\alpha_{r}$ is an effective overall distributed-loss coefficient per unit length. By assuming $T_{P}=T_{\mathrm{p} 1}=$ $T_{\mathrm{p} 2}$, then:

$R_{M}{ }^{2} T_{P}^{2}=e^{-2 \alpha_{r} L}$

The ring-down time can be written as:

$\tau=\frac{1}{c \alpha_{r}}$

where $c$ is the speed of light. 
Table 2-4: Comparison of a measured light transmittance, calculated loss coefficient, and ring-down time with two ways: power meter and lock-in amplifier for different EW-CRDS configuration. The measured and calculated ring-down times were compared.

\begin{tabular}{|c|c|c|c|c|c|c|c|}
\hline \multirow{2}{*}{ EW-CRDS configuration } & \multicolumn{3}{|c|}{ Using power meter } & \multicolumn{3}{c|}{ Using lock-in Amplifier } & $\begin{array}{c}\text { Measured } \\
\text { ring- } \\
\text { down } \\
\text { time (ns) }\end{array}$ \\
\cline { 2 - 8 } & $T_{P} \%$ & $\alpha_{r}\left(\boldsymbol{m}^{-1}\right)$ & $\tau(\mathbf{n s})$ & $T_{P} \%$ & $\boldsymbol{\alpha}_{r}\left(\boldsymbol{m}^{-1}\right)$ & $\tau$ (ns) & \\
\hline $\begin{array}{c}\text { In axis cavity with } \\
\text { uncoated prism }\end{array}$ & $98.5 \pm 0.3$ & $\begin{array}{c}(15.2 \pm 0.1) \\
\times 10^{-3}\end{array}$ & $220 \pm 1$ & $98.7 \pm 0.3$ & $\begin{array}{c}(13.10 \pm 0.08) \\
\times 10^{-3}\end{array}$ & $253 \pm 2$ & $260 \pm 2$ \\
\hline $\begin{array}{c}\text { In axis cavity with coated } \\
\text { prism }\end{array}$ & $98.8 \pm 0.4$ & $\begin{array}{c}(12.1 \pm 0.1) \\
\times 10^{-3}\end{array}$ & $275 \pm 2$ & $99.0 \pm 0.3$ & $\begin{array}{c}(10.10 \pm 0.06) \\
\times 10^{-3}\end{array}$ & $330 \pm 2$ & $320 \pm 3$ \\
\hline $\begin{array}{c}\text { Brewster angle (off-axis) } \\
\text { cavity }\end{array}$ & $99.7 \pm 0.3$ & $\begin{array}{c}(3.05 \pm 0.02) \\
\times 10^{-3}\end{array}$ & $\begin{array}{c}(109 \pm 1) \\
\times 10\end{array}$ & $99.8 \pm 0.2$ & $\begin{array}{c}(2.05 \pm 0.01) \\
\times 10^{-3}\end{array}$ & $\begin{array}{c}(162 \pm 1) \\
\times 10\end{array}$ & $1,350 \pm 4$ \\
\hline
\end{tabular}

The ring-down time was measured experimentally for each configuration by the EWCRDS device, and it was compared with the calculated results. The results of the measured single-pass transmittance, calculated loss coefficient, calculated and measured ring-down times are recorded in Table 2-4.

It is clear that the values of the loss coefficients and the ring-down times were obtained theoretically by both methods are very close. Moreover, the calculated ring-down times with the two methods are in good agreement with the experimental results measured by EW-CRDS. These results revealed that the Brewster's angle configuration is the best design to work with and the dominant source of the optical losses in these studies is the reflection at the air/prism interface. 


\subsection{The Minimum Detectable Absorption coefficient Limit}

Improving the limits of absorbance detection is a main challenge in the most optical spectroscopic methods. The conventional absorbance techniques is based on the measurement of a small intensity difference $(\Delta I)$ on a large background signal $\left(I_{\mathrm{o}}\right)$; this means the obtainable sensitivity depends on the accuracy with which a small $\Delta I / I_{\mathrm{o}}$ can be measured [12] .

The absolute precision with which we can measure the ring-down time is limited by the noise in the system this noise can be deduced by using better experiment design such as stable light source, low loss optical components, and low noise photodetectors.

In this section, the minimum detectable absorption coefficient limit was determined and compared for each CRDS configuration that was used in this work. If we assume a large number of ring-down times were collected at a particular wavelength, then the average value of the collected ring-down times is $1 / \tau_{0}$ with standard deviation $\sigma$. The relative measurement error $(\Delta \tau / \tau)$ is given by:

$\frac{\Delta \tau}{\tau}=\frac{\sigma}{1 / \tau_{0}}$

where $\Delta \tau=\left(\tau-\tau_{0}\right)$. It is clearly observed that $\Delta \tau / \tau$ remains generally constant regardless of the value of the baseline ring-down time $\left(\tau_{0}\right)$. Thus, $\Delta \tau / \tau$ is considered to measure the cavity-ring down spectrometer performance.

The minimum detectable absorption coefficient $\left(\Delta \alpha_{\min }\right)$ is defined as [21]: 
$\Delta \alpha_{\min }=\frac{1}{c \tau_{0}}\left[\frac{\Delta \tau}{\tau}\right]$

where $\Delta \tau$ is the smallest detectable change in the ring-down time: which is usually defined as the $2 \sigma$ variation in the cavity ring-down time $\left(\tau_{0}\right)$.

Table 2-5: A minimum detectable absorption coefficient $\left(\Delta \alpha_{\text {min }}\right)$ for various cavity ring-down times of various CRDS configurations. It is clear that $\Delta \alpha_{\min }$ is linearly proportional with ring-down time.

\begin{tabular}{|c|c|c|c|}
\hline CRDS configuration & $\begin{array}{l}\text { Ring-down time } \\
\qquad\left(\tau_{\circ}\right)(\mu s)\end{array}$ & $\begin{array}{l}\text { Error in } \tau_{\circ} \\
(\mu \mathbf{s})\end{array}$ & $\begin{array}{l}\Delta \alpha_{\min } \\
\left(c m^{-1}\right)\end{array}$ \\
\hline Empty cavity. & 60 & 0.2 & $1.9 \times 10^{-9}$ \\
\hline $\begin{array}{l}\text { EW Linear cavity configuration, using } \\
\text { uncoated prism. }\end{array}$ & 0.260 & 0.001 & $5 \times 10^{-7}$ \\
\hline $\begin{array}{l}\text { EW Linear cavity configuration, using } \\
\text { coated prism. }\end{array}$ & 0.320 & 0.001 & $3.2 \times 10^{-7}$ \\
\hline EW Brewster angle configuration. & 1.3 & 0.003 & $5.9 \times 10^{-8}$ \\
\hline $\begin{array}{l}\text { Linear cavity with normal incidence } \\
\text { cuvette. }\end{array}$ & 3.6 & 0.04 & $1.03 \times 10^{-8}$ \\
\hline $\begin{array}{l}\text { ITO ultra-thin film on Brewster's } \\
\text { Angle Substrates. }\end{array}$ & 1.6 & 0.002 & $2.6 \times 10^{-8}$ \\
\hline
\end{tabular}


From Equation 2-8, it is clear that the unit of $\Delta \alpha_{\min }$ is $\mathrm{cm}^{-1}$; thus, $\Delta \alpha_{\min }$ can be defined as the minimum detectable amount of optical loss in $1 \mathrm{~cm}$ of optical path length. Moreover, $\Delta \alpha_{\min }$ is strongly affected by the reflectivity of the mirrors, cavity alignment, small changes in cavity length, and imperfect ring-down fitting. This means $\alpha_{\min }$ is proportional to $1 / \tau_{0}$; the longer cavity ring-down time (with assuming a constant noise level) yields to more sensitive measurement. The results of the measured ring-down time $\left(\tau_{0}\right)$ and the $\Delta \alpha_{\min }$ of all CRD configurations are recorded in Table 2-5.

As we observe from Table 2-5, the sensitivity of our apparatus is in the range of 0.1-3 ppm, which is much higher compared to the conventional single-pass methods [ 71 , 79].

\subsection{Conclusions}

The designs, performance of each condensed phase CRDS configuration, and spectroelectrochemical data acquisition system were described. For the EW-CRDS instrument performance, the optical loss measurements revealed that the main source of loss is the reflections at air/prism interface and Brewster's angle configuration is the best geometry to work with. Finally, the performance of each condensed phase CRDS configuration was assessed by comparing the minimum detectable absorption coefficient limit. Overall, all CRDS platforms provided high sensitivity for optical absorption measurements. 


\section{CHAPTER 3}

\section{AN ELECTRO-ACTIVE INTERFACE FOR EVANESCENT-WAVE CAVITY-RING- DOWN SPECTROSCOPY: EXPANDING ITS CAPABILITY FOR ELECTROCHEMICAL AND ADSORPTION KINETIC INVESTIGATIONS}

\subsection{Introduction}

Proteins are complex bio-macromolecules that are intrinsically surface-active. Understanding the redox processes and controlling the adsorption of redox active proteins at the solid/liquid interface plays an essential role in developing many applications such as: bio-sensing technology, food and drugs manufacturing, performing enzymatic catalysis and other various bioelectronics applications [58-62, 80-82]. The capability of the highly sensitive EW-CRDS platform to address the solid/liquid interfaces can be extended to optically investigate redox events. This chapter addresses the development of an electroactive interface, which is directly integrated to the EW-CRDS platform, in order to expand its spectroelectrochemical capabilities to investigate properties of surface-adsorbed redox species. A highly optical transparent and electrically conductive thin film electrode has been incorporated into the TIR interface. A high optical transparency of the thin film electrode is required to maintain long ring-down times and preserve the high sensitivity of the EW-CRDS platform for absorbance measurements. High conductivity is concurrently needed to enable time-varying electrochemical controls. 
The first section presents a description of preliminary evidence showing the platform capabilities. The electro-active EW-CRDS was used to study the adsorption isotherm and the redox properties of the immobilized redox-active Cyt-C protein under potential modulation.

The second section highlights the capability of the electro-active EW-CRDS device to follow dynamic processes in real time. Three sets of data are reported here. First, we present measurements performed at open electric potential conditions for the adsorption process of a heme protein (Cyt-C) onto different types of solid/liquid interfaces, namely indium tin oxide (ITO), 3-Aminopropyl Triethoxysilane (APTES), and Cyt-C antibody (Ab). In the next set of data, an electric potential was applied to the adsorbent surface and fixed at discrete of values. For each value of the applied potential the adsorption properties of the Cyt-C redox protein binding to the different interfaces mentioned above were measured. Finally, we used a cyclic voltammetry (CV) scanning protocol and performed simultaneous optical measurements with the EW-CRDS tool to optically follow the Cyt-C adsorption onto the same set of interfaces under electric potential modulation. To properly describe the protein binding properties, several parameters were determined from our experimental data: the surface association $\left(k_{a}\right)$ and dissociation $\left(k_{d}\right)$ rate constants, the equilibrium binding constant $\left(k_{e q}\right)$, and the Gibbs free energy of adsorption $(\Delta G)$. 


\subsection{Probing Redox Reactions of Immobilized Cyt-C Protein}

For a proof of concept demonstration, the electro-active EW-CRDS technique was applied to determine the surface coverage of and to investigate the redox process of the directly adsorbed Cyt-C protein on ITO electrode under potential steps.

\subsubsection{EW-CRDS Experimental Setup}

Figure 3-1(a) shows a schematic representation of the experimental setup. A linear (in-axis) confocal cavity is formed by two identical high-reflectivity concave mirrors, with a diameter of $2.54 \mathrm{~cm}$ and a $1 \mathrm{~m}$ radius of curvature $(\mathrm{R}=99.995 \%$ at $532 \mathrm{~nm}, \mathrm{CRD}$ Optics, Inc.), separated by a distance of $100 \mathrm{~cm}$ and mounted on adjustable mounts (CRD Optics, Inc.).

The cavity excitation source was the second harmonic of the Nd:YAG pulsed laser ( Surelite II, Continuum) operating at $532 \mathrm{~nm}$, repetition rate $10 \mathrm{~Hz}$, and pulse duration of about 4 - $6 \mathrm{~ns}$. The p-polarized radiation was selected by guiding the laser beam through a half wave plate (AHWP05M-600, Thorlabs), and a linear polarizer.

In order to create the electro-active interface of EW-CRDS platform, ITO $(13 \mathrm{~nm})$ was deposited on the glass substrate using a DC magnetron sputtering technique (see details in section 3.2.2). As shown in Figure 3-1(b), the ITO-coated glass substrate was mounted in a homemade electrochemical flow cell with a three-electrode configuration and connected to a standard $20 \mathrm{~mm} \mathrm{N-BK7}$ antireflection-coated right angle prism (PS908LA, Thorlabs) using index matching gel $(n=1.52)$. The ITO film served as the working electrode (WE), a $\mathrm{Ag} / \mathrm{AgCl}$ electrode in $1 \mathrm{M} \mathrm{KCl}$ solution was used as reference (RE) and 
platinum wire as counter electrodes (CE). A potentiostat (CHI 660D, CH Instruments, Inc.) was connected to the three electrodes to apply and control the potential at the working electrode. The flowcell was mounted on 3-D translation stage (XR25P-K2, Thorlabs) and placed symmetrically in the center of the cavity.

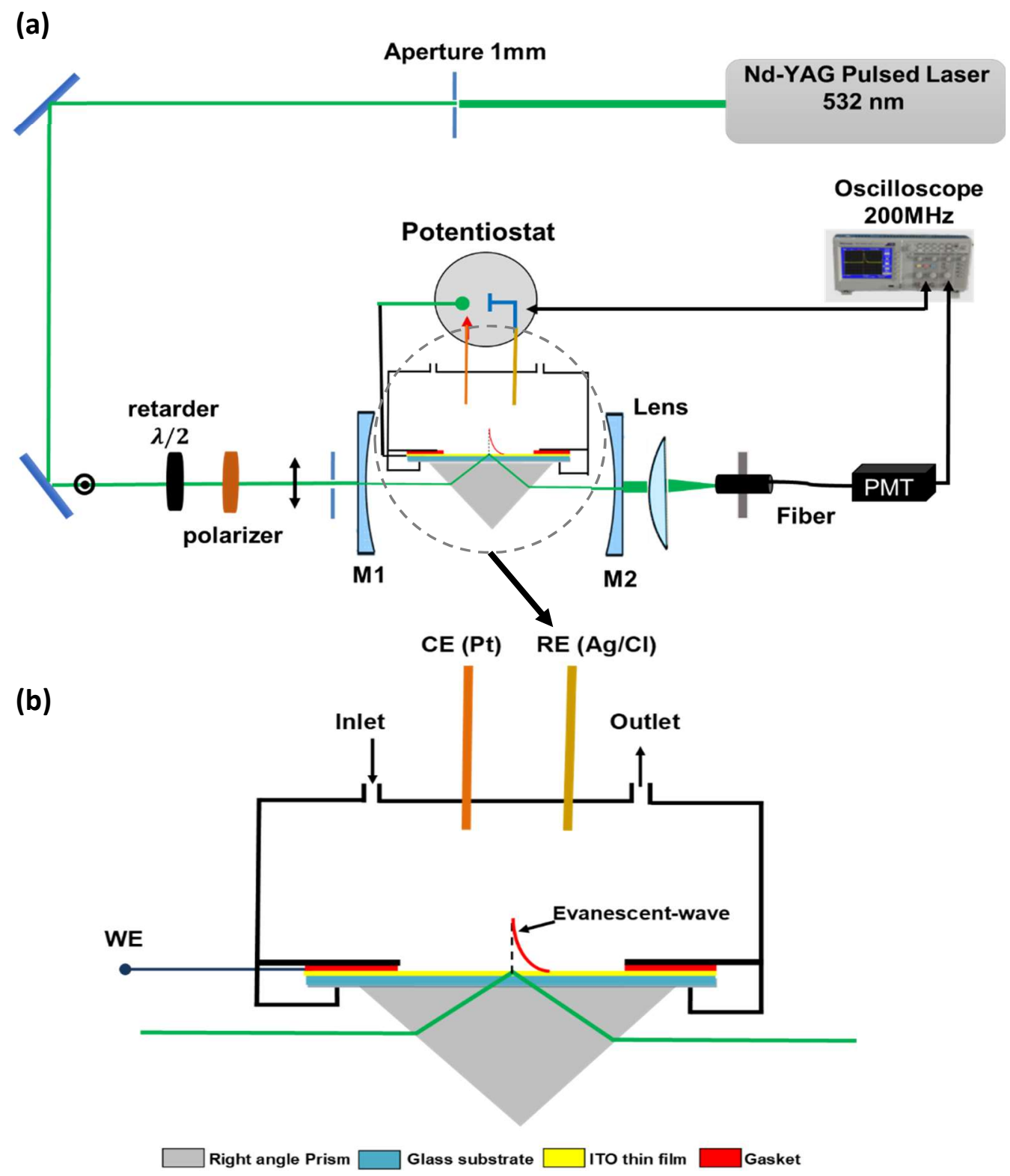

Figure 3-1: (a) Schematic diagram of the experimental setup for the EW-CRDS. (b) Enlarged picture of electrochemical flow cell and prism. 
Before entering the laser cavity, the laser beam was spatially filtered by two irises (aperture diameter of $1 \mathrm{~mm}$ ). The light that leaks out of the cavity was focused into an optical fiber (P600-1-UV-VIS, Ocean Optics) by a focusing lens and directed into a PMT (H5783-4, Hamamatsu). No other device was determined to be necessary for spatial filtering of the optical beam. The ring-down signal from the PMT is recorded using a digital oscilloscope (2Gsa/s, 200MHz bandwidth, DSO1022A, Agilent). Typically, 16 laser shots (at $10 \mathrm{~Hz}$ ) were averaged in the oscilloscope before data were downloaded. To extract ring-down time, a Python program was used to fit experimental data using an exponential decay function (Python program code is described in appendix 2).

\subsubsection{ITO Ultra-Thin Film Sample Coating}

The cleaning and ITO coating processes are critical for reducing optical loss. Surface preparation began with the cleaning of the prism or glass substrate to remove organic and inorganic contamination. This procedure was accomplished by sequentially sonicating the prism device in Triton soap (wiped with cotton swabs), deionized (DI) water, and piranha solution, followed by rinsing with DI water, acetone, and methanol for 15 minutes each time, and dried with $\mathrm{N}_{2}$ gas. For very low optical losses and long cavity ring-down times (and thus, enhanced sensitivity) the electro-active interface of the EW-CRDS platform requires an electrode film with extremely low optical losses (the imaginary part of the refractive of the electrode film should be on the order of $2 \times 10^{-3}$, or lower). On the other hand, the electrochemical capability aimed for the platform also demands very low resistivity as well for such film (resistivity on the order of about $2 \times 10^{-3} \Omega . \mathrm{cm}$ or lower). While working towards another challenging application (electro-active, single-mode, 
integrated optical waveguides) we have demonstrated in our labs a technology for growing ITO films that simultaneously feature the high performances described above, which far surpasses the requirements common for transparent electrodes in display and other technologies. We have demonstrated that when a precise set of fabrication conditions are followed, ultra-low optical losses and still conductive ITO films can be obtained. Those fabrication steps have been described in details by us elsewhere[83].

After a high base vacuum, $10^{-7}$ Torr, a DC sputtering technique with reactive atmosphere of $\mathrm{O}_{2}$ was utilized to coat an ITO film ( $\sim 13 \mathrm{~nm}$ thickness $)$ on the hypotenuse surface of the prism, which was maintained at $250{ }^{\circ} \mathrm{C}$ during deposition. To promote the electrical conductivity and optical transparency, the ITO film was submitted to a postdeposition plasma annealing process, which featured UV (264 nm) radiation at atmospheric air conditions with the substrate heated at $200-250{ }^{\circ} \mathrm{C}$ for 1 hour [83].

\subsubsection{Absorbance and Surface Coverage Calculations}

For a ring cavity as described in Figure 3-1, the interfacial optical absorbance (A) of the light onto the sample can be calculated by using the equation:

$$
A=\frac{\left(n_{a} L+n_{p} l\right)}{\ln (10) c}\left(\frac{1}{\tau}-\frac{1}{\tau_{0}}\right)
$$

where $L$ is the light pulse pathlength in air inside the cavity, $l$ is the pathlength inside the prism, $n_{a}\left(n_{p}\right)$ is the refractive index of air (prism), c is the speed of light, and $\tau_{0}$ and $\tau$ are the cavity ring-down times with thebackground (buffer) and the analyte of interest present, 
respectively. Absorbance can be converted into the surface density (Г) of adsorbed species and can be determine by the following relation:

$$
\Gamma=\frac{A}{S \epsilon}
$$

where $\epsilon$ is the molar absorptivity of the adsorbed analyte and $S$ is a sensitivity factor of the evanescent-wave, for the transverse magnetic (TM) polarization S is given by:[84]

$$
S=4 n_{c} n_{p}^{2}\left(n_{p}^{2}-N_{e f f}^{2}\right)^{\frac{1}{2}} \frac{\left[2 N_{e f f}^{2}-n_{c}^{2}\right]}{\left[n_{p}^{4}\left(N_{e f f}^{2}-n_{c}^{2}\right)+n_{c}^{4}\left(n_{p}^{2}-N_{e f f}^{2}\right)\right.}
$$

where $n_{c}$ and $n_{p}$ are the refractive indices of water and prism, respectively, $N_{e f f}$ is the effective refractive index $\left(N_{\text {eff }} \equiv n_{p} \sin \theta_{p}\right)$, and $\theta_{p}=72.8^{\circ}$ is the angle of incidence from inside the prism. For the current setup, the sensitivity factor was determined to be $S=5.6$. As we deployed a laser with a wavelength of $532 \mathrm{~nm}$, only the Cyt-C protein exhibited strong absorption transition at this wavelength. At the oxidized state of the protein, we have $\epsilon_{O x}=10.05 \times 10^{3} \mathrm{M}^{-1} \mathrm{~cm}^{-1}$.

\subsubsection{Results and Discussion}

3.2.4.1. Determination the Surface Coverage of Adsorbed Cyt-C Protein on Glass and ITO Surfaces

To investigate the adsorption of oxidized Cyt-C kinetics at the glass/water and ITO/water interfaces, phosphate buffer saline (PBS) was first introduced into the flow cell for collection of a background ring-down time $\left(\tau_{\circ}\right)$. Next, different concentrations of CytC solution, in the range of 0.02-7 $\mu \mathrm{M}$, were prepared in a PBS buffer of PH-7 (see appendix 
3 for Cyt-C preparation) and injected to the flow cell, causinga reduction in the characteristic ring-down time $(\tau)$. A waiting time of typically $10-15$ minutes is needed to ensure that the protein surface adsorption has reached equilibrium. The absolute interfacial absorbance was calculated using Equation 3-1 and converted to surface coverage using Equation 3-2.

Figure 3-2 shows adsorption isotherms of oxidized Cyt-C on ITO surface. Each of the data sets shown in Figure 3-2 were fitted to a Langmuir adsorption model:

$\Gamma=\frac{K_{e q} \Gamma_{s a t} C}{1+K_{e q} C}$

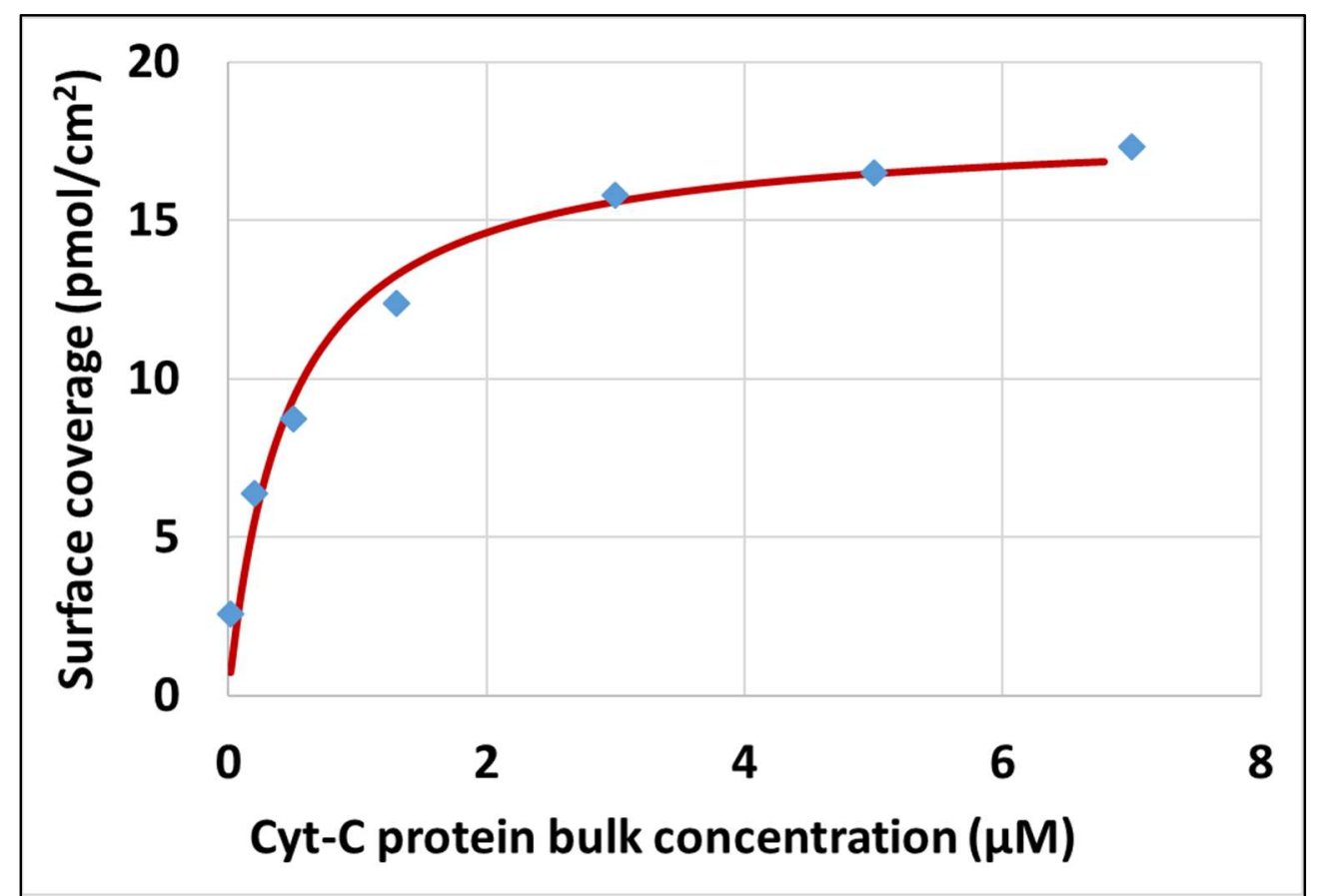

Figure 3-2: Adsorption isotherm of Cyt-C on ITO surface at pH-7, measured at 406 $\mathrm{nm}$. The dashed curve is the best fit of the experimental data to a Langmuir adsorption model 
where $\Gamma$ is is the surface coverage resulted from a bulk concentration of the protein, $K_{e q}$ is the adsorption equilibrium constant, $\Gamma_{s a t}$ is the saturated surface coverage, and $C$ is the concentration in bulk solution. From the fitting results, $\Gamma_{\text {sat }}$ are $\left(15 \pm 1\right.$ pmol. $\left.\mathrm{cm}^{-2}\right),(18 \pm 1$ pmol. $\mathrm{cm}^{-2}$ ) on bare glass and ITO respectively, which correspond to about $68 \%, 86 \%$ of a closely packed monolayer about 22 pmol. $\mathrm{cm}^{-2}[59,85]$.

The values of the equilibrium constants are $K_{e q}=(2.0 \pm 0.4) \times 10^{6} M^{-1}$ (on glass) $K_{e q}=(2.3 \pm 0.4) \times 10^{6} M^{-1}$ (on ITO), which are in agreement with what was previously reported for the Cyt-C adsorption on ITO surface [86].

3.2.4.2. Probing Redox Reactions of Immobilized Cyt-C under a Step Potential Modulation

The redox process of Cyt-C protein was investigated by potential steps modulation. The potential between the $\mathrm{Ag} / \mathrm{AgCl}$ reference electrode and the ITO working electrode was stepped from $-0.5 \mathrm{~V}$ to $0.5 \mathrm{~V}$, and the reference optical signals (ring-down times) were first collected without the presence of Cyt-C in the flow cell. Then after 15 minutes of relaxation time, $500 \mathrm{nM}$ Cyt-C solution was injected into the flow cell; another 30 minutes was used to stabilize the adsorption of Cyt-C on the surface of ITO. Then the electrochemical flow cell was rinsed with PBS buffer to remove unbound species. Finally, the potential was stepped again with Cyt-C proteins present in the flowcell, and the ring-down time was recorded. The values of the ring-down times with and without Cyt-C were then used to generate the absorbance plot utilizing Equation 31. The EW-CRDS response under step potentials is shown in Figure 3-3. The optical data show the strong transition of optical absorbance as the applied potential crosses the 
formal redox potential of $\mathrm{Cyt}-\mathrm{C}$; the formal potential can be estimated by fitting the modulated absorbance (red curve) and its derivative (black curve) as a function of the applied potential and it was found to be approximately $70 \mathrm{mV}$.

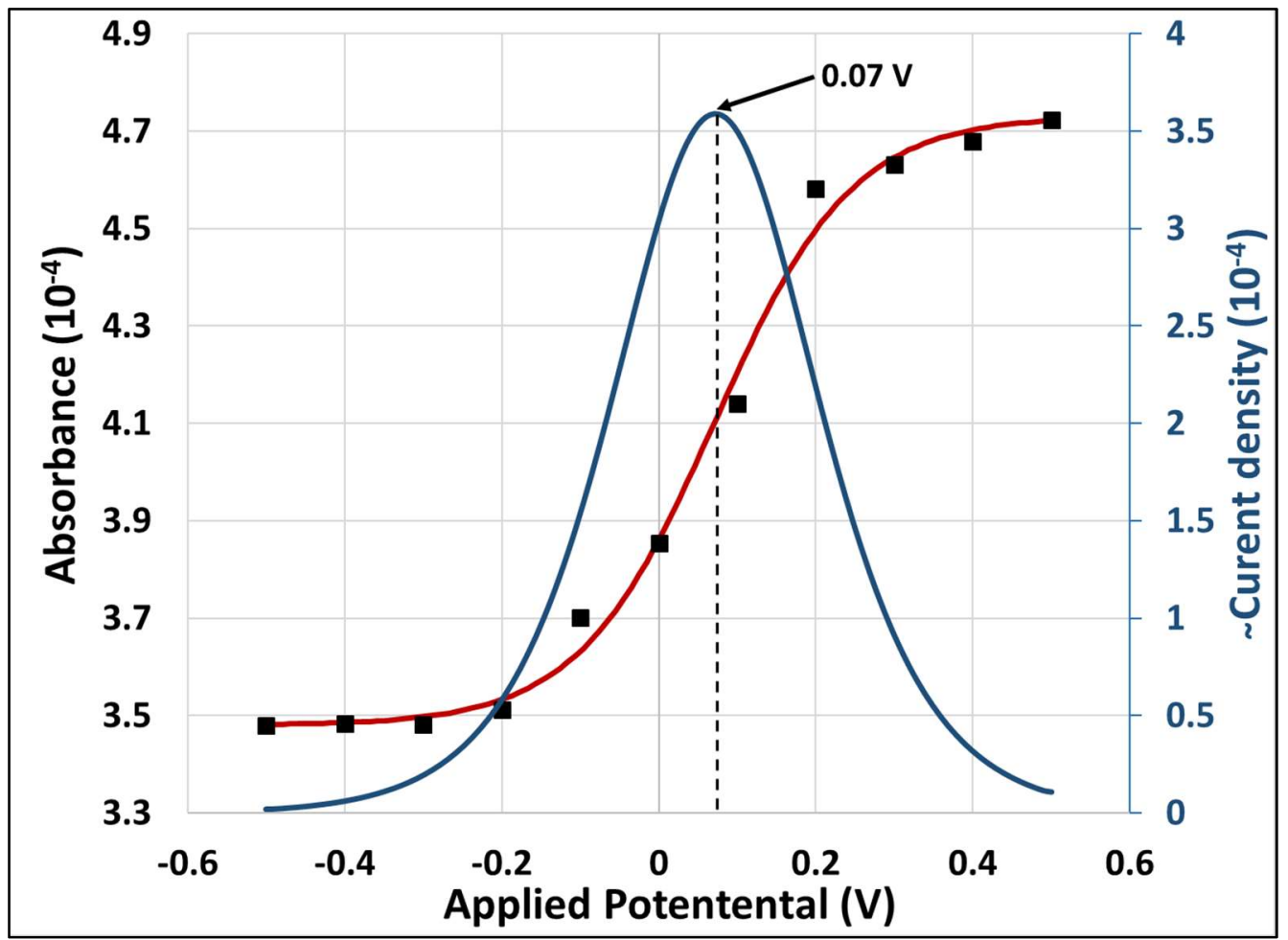

Figure 3-3: Absorbance data (black data points) as measured by the EW-CRDS platform at $532 \mathrm{~nm}$ for an adsorbed sub-monolayer of Cyt-C protein undergoing step potential modulation (from $-0.5 \mathrm{~V}$ to $0.5 \mathrm{~V}$ )on ITO surface, with a curve fitting (red curve) and the derivative of the fitted line (blue curve) to determine the formal potential (here the blue trace is only proportional to the current density).

At positive values of potential in Figure 3-3, Cyt-C molecules are fully oxidized with the total surface density $\left(\Gamma_{\text {tot }}=\Gamma_{O x}\right)$, which can be calculated using Equation $\Gamma_{O X}=A_{O x} /\left(\mathrm{S} \epsilon_{O x}\right)$, where $A_{O x}$ is the measured absorbance of Cyt-C in its oxidized state, $\mathrm{S}$ is the sensitivity factor of EW-CRDS device, and $\epsilon_{O x}$ is the molar absorptivity of Cyt- 
$\mathrm{C}$ in its oxidized state at $532 \mathrm{~nm}$. Such calculation resulted in a value of $\Gamma_{O x}=(8.3 \pm$ 0.1) pmol. $\mathrm{cm}^{-2}$ which corresponds to about $38 \%$ of a full monolayer on ITO. As the potential goes toward negative values, the protein starts to reduce. In order to calculate the fraction of Cyt-C species that were converted to the reduced state, Equation 3-2 was modified to include the possible co-presence of different redox species on the surface:

$$
A_{\text {red }}=S\left[\epsilon_{\text {ox }}\left(\Gamma_{\text {tot }}-\Gamma_{\text {red }}\right)+\epsilon_{\text {red }} \Gamma_{\text {red }}\right]
$$

where $\Gamma_{t o t}$ is the total surface coverage of Cyt-C in its oxidized state $\left(\Gamma_{t o t}=\Gamma_{o x}\right), \Gamma_{r e d}$ is the surface density of the immobilized protein species that are converted to the reduced state, and $\epsilon_{\text {red }}$ is the molar absorptivity of the Cyt-C protein at the reduced state, with $\epsilon_{\text {red }}=7 \times 10^{3} \mathrm{M}^{-1} \mathrm{~cm}^{-1}$ at $532 \mathrm{~nm}$. The data of absorbance in a reduced state $A_{\text {red }}$ (displayed in Figure 3-3) at applied potential values smaller than the redox transition was then considered to determine $\Gamma_{\text {red }}$ by solving Equation 3-5 as following:

$\Gamma_{\text {red }}=\left(\frac{A_{\text {red }}}{\mathrm{s}}-\epsilon_{\text {ox }} \Gamma_{\text {tot }}\right) /\left(\epsilon_{\text {red }}-\epsilon_{o x}\right)$

These calculations give us the value $\Gamma_{\text {red }}=7.0 \pm 0.1 \mathrm{pmol} . \mathrm{cm}^{-2}$. The fraction of reduced species over total surface density $\left(\Gamma_{\text {red }} / \Gamma_{\text {tot }}\right)$ that undergo redox transition during a redox process was found to be $84 \%$ of the total surface coverage. 
3.3. Real-Time Monitoring of Adsorption Kinetics under Potential Control and Following the Redox Processes of Cyt-c Protein Using Electro-Active EW-CRDS

\subsubsection{Experimental setup.}

The experimental setup (as shown in Figure 3-4(a)) used was the same as described in Section 3.2.1. The difference here is that the laser beam enters the cavity through the back of highly reflective mirror and hits the surface of the prism close to the edge at Brewster's angle. A standard 20 mm N-BK7 uncoated right angle prism (PS908, Thorlabs) was used.

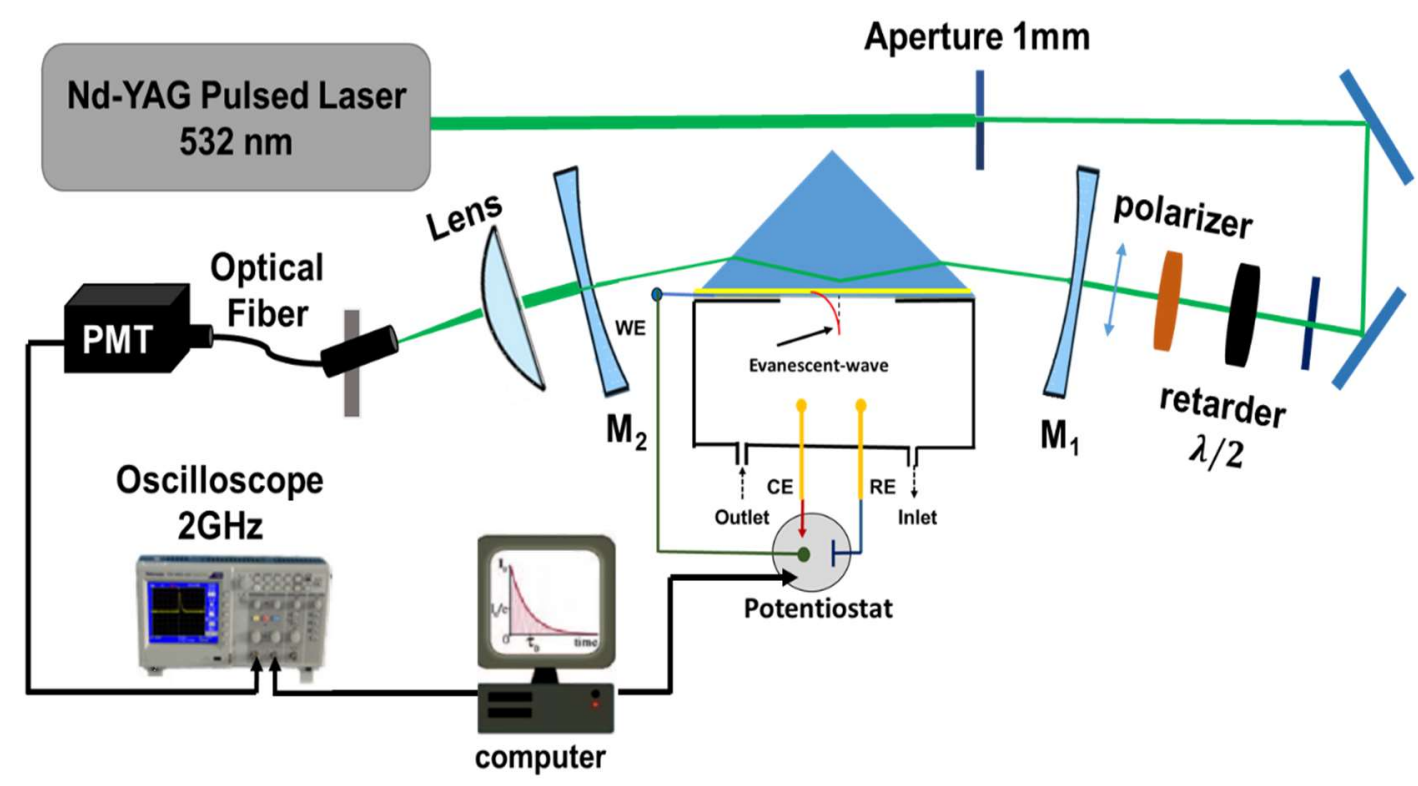

Figure 3-4: Schematic diagram of experimental setup for EW-CRDS measurement. The spectro-electrochemical flow cell attached to the TIR surface.

The light that leaks out of the cavity was focused into an optical fiber by a focusing lens and directed into a PMT. The ring-down signal from the PMT was recorded using a digital oscilloscope (4Gsa/s, $1 \mathrm{GHz}$ bandwidth, DSO8104A Infiniium, Agilent). The data 
was transferred into a computer via a GPIB to Ethernet interface connection (GPIBENET/1000, National Instruments, Austin, TX). The averaged ring down traces (typically between $8-16$ laser pulses) were used to calculate the ring down time $\tau$, using A leastsquares fitting routine via a custom- LabVIEW program that orchestrated the synchronization of the optical and electrochemical data.

The ring-down times are limited by the scattering and diffraction losses at the surfaces of incidence and the EW-surface of the prism, but not by the mirror reflectivity $[63,64]$. Our current setup produced cavity ring-down times of approximately $(1.4 \pm 0.01) \mu s$, the ring-down time of the cavity decreased to $(0.9 \pm 0.01) \mu$ s upon coating the TIR interface of the prism with an electro-active film of ITO (thickness of about 13 $\mathrm{nm}$, see details in section 3.2.2).

The ITO-coated prism was then mounted in a homemade micro-electrochemical flow cell with a three-electrode configuration and spatially adjusted by a 3-D translation stage (XR25P-K2, Thorlabs). The ITO film served as the working electrode (WE), while two gold-plated pins (surface area of 2.85 $\mathrm{mm}^{2}$, Mouser Electronics, 8451-0-00-34-00-00-330) were used as reference (RE) and counter pseudo- electrodes (CE). A commercial potentiostat was utilized for the electrochemical control. 


\subsubsection{Functionalization Protocols of EW-CRD Surface.}

Immobilization protocols of immunolayers on the EW-CRDS surface are crucial for the adsorption process.

\subsubsection{APTES Functionalization}

The ITO-coated prism was functionalized with APTES (purchased from Sigma Aldrich) by immersing the prism in a solution containing $10 \mathrm{~mL}$ of 2-propanol, $100 \mu \mathrm{L}$ of APTES, and $4-5$ drops of DI water. The device surface was then thoroughly rinsed with 2-propanol and DI water, and dried under $\mathrm{N}_{2}$ gas. In addition to the chemical adsorption process, species of APTES can also physically adsorb to the working surface. To remove those loosely bound APTES species, the prism was sonicated in DI water for 15 minutes, rinsed with DI water, and dried under $\mathrm{N}_{2}$ gas $[87,88]$.

To access the immobilization process of APTES onto the ITO film, glass slide samples were coated with a similar ITO film and incubated with APTES as described above. Three different incubation times were tested: $1 \mathrm{~h}, 3 \mathrm{~h}$, and $6 \mathrm{~h}$. The three samples were then exposed to a PBS solution of Cyt-C antibody $(2 \mu \mathrm{g} / \mathrm{ml})$ that had been labeled with the Alexa fluorescent probe (Cytochrome C (A-8) mouse monoclonal IgG2b 158 Alexa fluorescent 647, Santa Cruz Biotechnology). Then the slides were rinsed with DI

water to remove unbound species and dried under a stream of $\mathrm{N}_{2}$ gas. Optical imaging of the molecular layer using confocal fluorescent microscopy provided a means to determine the presence and reactivity of the APTES layer to the ITO film. 
The uniformity of the fluorescent-labeled Ab on the ITO/APTES surface was tested under confocal fluorescence imaging with the results illustrated in Figure 3-5. Figure 35(c) shows that an incubation time of $6 \mathrm{~h}$ is adequate to form a uniform layer of APTES to the glass/ITO surface. An incubation time of 6 hours was adopted for functionalizing the ITO-coated prism with the APTES layer
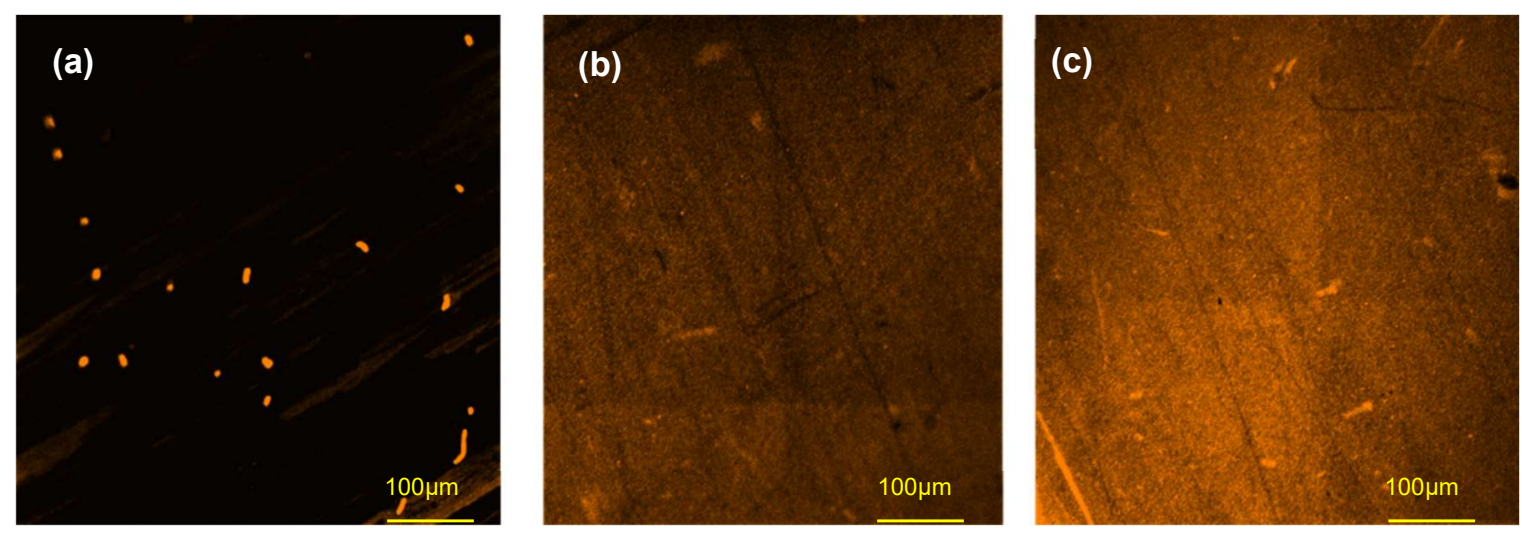

Figure 3-5: Confocal fluorescence image of the ITO/APTES EW-CRDS surface when exposed to Cyt-C-Ab that has been previously labeled with an Alexa fluorescent probe. Each panel corresponds to a different incubation time of the APTES onto the ITO surface (a) $1 \mathrm{~h}$, (b) $3 \mathrm{~h}$, (c) $6 \mathrm{~h}$.

\subsubsection{Primary Cyt-C Capturing Antibody Functionalization}

For experiments involving the adsorption of Cyt-C to the prism/ITO/APTES/Cyt$\mathrm{C}-\mathrm{Ab}$ assembly, the prism/ITO/APTES surface was functionalized with Cyt-C-Ab (Cyt-C antibody (A-8) mouse monoclonal IgG2b, Santa Cruz Biotechnology) by injecting a PBS solution of Cyt-C-Ab at a concentration of $2 \mu \mathrm{g} / \mathrm{mL}$ into the flowcell. After an incubation period of one hour, the flowcell was rinsed with PBS solution to remove unbound species. 
The stability of the Cyt-C-Ab binding to the APTES surface was confirmed at different applied potentials (see details in appendix 4).

\subsubsection{Recycling the EW-CRDS Surface}

The interaction between the capture antibody and the ITO/APTES can be reversed by sonicating the prism surface in a potassium carbonate solution ( $\mathrm{pH} 9-11)$ to easily renew the sensing interface for the next set of experiments [88]. Between each set of measurements, the prism was re-cleaned using the protocols described in section 3.2.2.

\subsubsection{Binding Kinetics of Cyt-C Protein Model}

The model of adsorption of Cyt-C protein from the bulk solution phase onto the device surface can be described by $[89,90]$ :

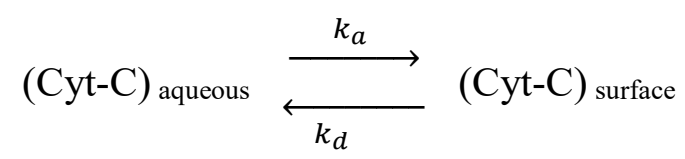

where (Cyt-C)aqueous represents Cyt-C species dissolved in the bulk aqueous solution and $(\mathrm{Cyt}-\mathrm{C})_{\text {surface }}$ accounts for surface-adsorbed species, $k_{a}$ is the association rate constant, and $k_{d}$ is the dissociation rate constant. The binding rate can be expressed by [91]:

$$
\frac{d \Gamma}{d t}=k_{a} \frac{C}{C_{w}} \Gamma_{w}-k_{d} \Gamma
$$

where $c$ is the protein bulk solution concentration, $C_{w}=55.5 \mathrm{M}$ is the water molarity, $\Gamma$ is the protein surface density, and $\Gamma_{w}$ is the surface density of vacant sites (protein-free surface sites). Following, $\Gamma_{\text {tot }}=\Gamma+\Gamma_{w}$ is constant and Equation 3-7 becomes 


$$
\frac{d \Gamma}{d t}=-\left(\frac{k_{a}}{c_{w}} c+k_{d}\right) \Gamma+k_{a} \frac{C}{C_{w}} \Gamma_{t o t}
$$

As expressed by Equation 3-2, the optical absorbance is linearly proportional to the protein surface density $\Gamma$. One can measure the optical absorbance as it changes over time using Equation 3-1. Therefore, from Equation 3-8, a plot of $\frac{d \Gamma}{d t}$ versus $\Gamma$ should feature a straight line with a slope $(\Delta m)$ given by $\Delta m=-\left(\frac{k_{a}}{c_{w}} c+k_{d}\right)$. Then, by collecting data for several Cyt-C bulk solution concentrations, $c$, and creating a plot of $\Delta m$ versus $c$, a straightline fitting to the data will have a slope and $y$-intercept equal to $-\frac{k_{a}}{c_{w}}$ and $-k_{d}$, respectively. The corresponding Gibbs adsorption free energy can then be obtained from the equilibrium constant $K_{e q}=\frac{k_{a}}{k_{d}}$ by using [92]:

$$
\Delta G=-R T \ln \left(K_{e q}\right)
$$

where $R=8.314 \mathrm{~J} . \mathrm{K}^{-1} \mathrm{~mol}^{-1}$ and $T=295 \mathrm{~K}$ are the gas constant and the absolute room temperature, respectively. 


\subsubsection{Results and Discussion}

\subsubsection{EW-CRDS of Protein Adsorption at Different Surfaces under Open Potential Condition}

As a preliminary demonstration of the ability of our platform to measure small values of optical absorbance and monitor real-time changes even at low submonolayer levels of an analyte, we investigated the adsorption kinetics of the Cyt-C protein at different molecular interfaces. In this first set of experiments, we operated under open-circuit potential (OCP) conditions (i.e., no electrical voltage applied in this particular set of data, although the ITO electrode film was present in the EW-CRDS device); under OCP conditions the equilibrium potential difference between working and reference electrodes was determined to be about $0.15 \mathrm{~V}$. The following concentrations of the Cyt-C protein solution were used: 50, 200, 500 and 1,000 nM; all protein solutions in PBS were at neutral $\mathrm{pH}$ conditions $(\mathrm{pH}=7.02)$. As described next, measurements were performed to determine the Cyt-C adsorption kinetics and binding properties on different assemblies (ITO, ITO/APTES, and ITO/Cyt-C antibody) attached to the glass surface of the prism undergoing internal reflection.

The experiment with the prism-coated ITO surface was performed in the following way. First, a PBS solution was introduced into the flowcell and a baseline signal of the ring-down time $\left(\tau_{0}\right)$ was recorded for 16 minutes using our custom-made LabView program; the average time was used to determine $\tau_{0}$. Then, upon adding the Cyt-C protein to the electrochemical flowcell, the ring-down time $(\tau)$ was immediately and continuously 
acquired for 16 minutes, which was experimentally observed to provide adequate time to reach a plateau in the protein adsorption process.

Figure 3-6(a) features the experimental kinetic data of the surface density of the Cyt-C protein, where each trace corresponds to a different concentration of the solution deployed. Each data point in each trace was calculated using Equation 3-1, which includes of the ring-down time before $\left(\tau_{0}\right)$ and during $(\tau)$ protein adsorption to determine the timedependent optical absorbance $A(t)$. Equations 3-2 and 3-3 were then used to obtain from the optical absorbance data the time evolution of the surface coverage, $\Gamma(t)$, where the sensitivity factor was determined to be $S=3.78$ for the current setup. It is worth noticing that a closely-packed full monolayer corresponds to about $22 \mathrm{pmol} \mathrm{cm}^{-2}$, and our kinetic experimental data only involves a small fraction of a monolayer.

To calculate the binding properties of Cyt-C adsorbing onto the ITO surface, for each bulk protein concentration, $c_{s}$, the experimental surface coverage against time was fitted with an exponential curve, $\Gamma(t)=\Gamma_{\infty}\left[1-e^{-a(t-b)}\right]$; these fitting curves are included in Figure 3-6(a). Then, the derivative of each function describing the time-varying surface coverage time, $\Gamma(t)$, was used to determine the binding rate, $\frac{d \Gamma(t)}{d t}$. In Figure 3-6(b), the binding rate, $\frac{\mathrm{d} \Gamma(t)}{d t}$, versus the surface coverage, $\Gamma(t)$, are displayed for each bulk protein concentration, c. This plot gave us the slope, $\Delta m$, for each protein solution concentration. Then, the negative value of each slope, $-\Delta m$, was plotted against the corresponding bulk protein concentration, and the results are shown in Figure 3-6(c). 

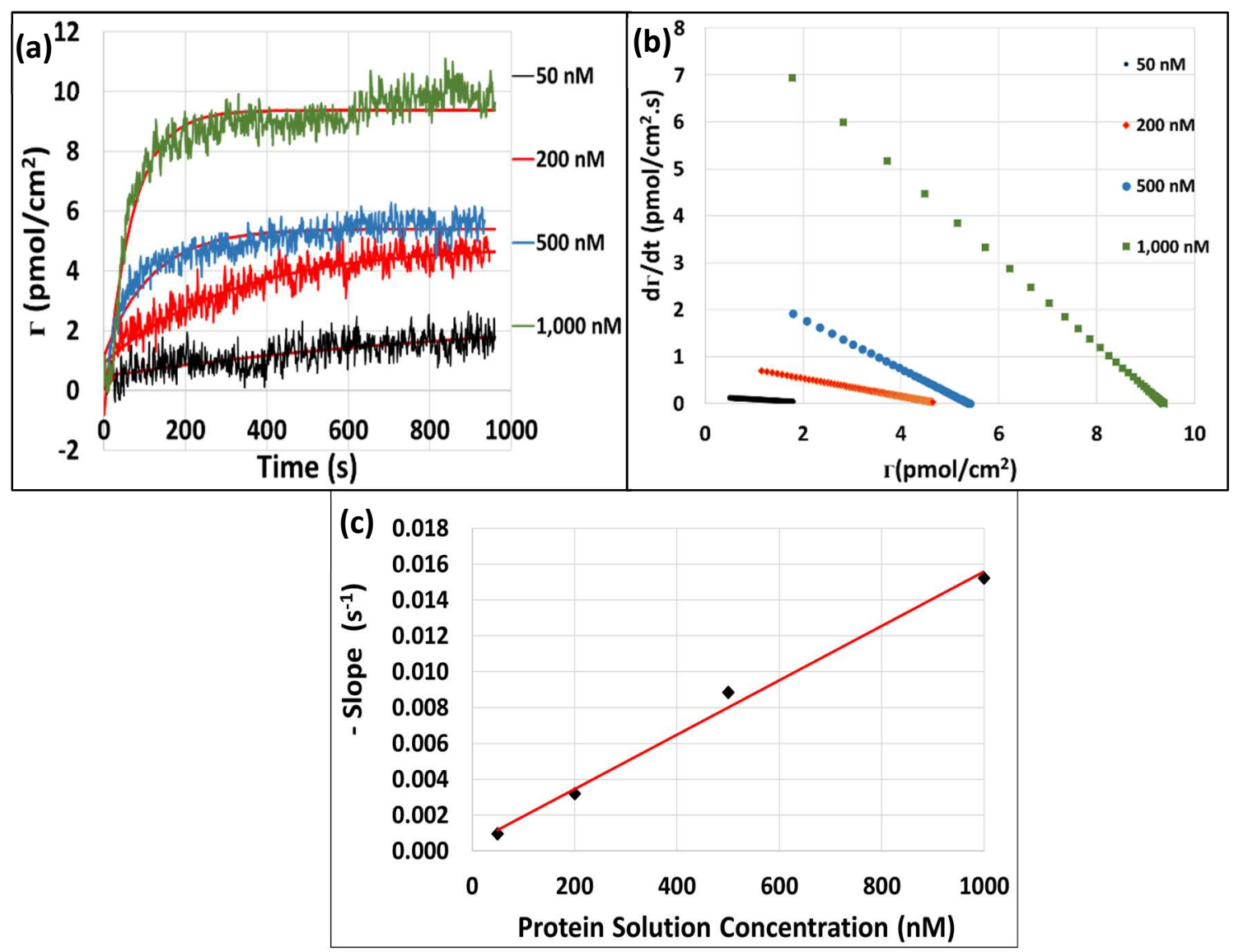

Figure 3-6: (a) Surface coverage change of Cyt-C binding on the bare ITO measured by EW-CRDS platform for different concentrations in real-time. (b) The plot of the binding rate versus bounded surface coverage for different Cyt-C concentrations. (c) The plot of the slope values was obtained in Figure 3-6(b) and plotted against Cyt-C concentration, to determine the association and dissociation constants.

A linear fitting of such data allows us to determine the association, $k_{a}$, and dissociation, $k_{d}$, binding constants from the slope and the y-intercept, respectively. The experimental results of the EW-CRDS platform for the association and dissociation rate constants, the equilibrium constant and the Gibbs free energy $(\Delta G)$ of Cyt-C interacting with the ITO surface are included in Table 3-1. These results are very close and in good 
agreement with what was previously reported for the Cyt-C adsorption on glass surface [91].

Following similar protocols of data acquisition and analysis, the Cyt-C protein adsorption onto different assemblies (ITO/APTES and ITO/Cyt-C Ab) were carried out. Table 3-1 summarizes the experimental results.

Table 3-1: The Association Rate Constant $\left(\mathrm{k}_{\mathrm{a}}\right)$, the Dissociation Rate Constant $\left(\mathrm{k}_{\mathrm{d}}\right)$, the Equilibrium Constant $\left(\mathrm{k}_{\mathrm{eq}}\right)$, and the Gibbs free energy $(\Delta G)$ for Cyt-C Binding to Different EW-CRDS Functionalized Surfaces.

\begin{tabular}{|c|c|c|c|c|}
\hline $\begin{array}{c}\text { EW-CRDS } \\
\text { functionalized surface }\end{array}$ & $k_{a}\left(10^{5} s^{-1}\right)$ & $k_{d}\left(10^{-4} s^{-1}\right)$ & $K_{\text {eq }}\left(10^{9}\right)$ & $\begin{array}{l}\text { Gibbs energy } \\
\left({\left.\mathrm{KJ} . \mathrm{mol}^{-1}\right)}^{-1}\right.\end{array}$ \\
\hline ITO & $8.4 \pm 0.5$ & $4.2 \pm 0.6$ & $2.0 \pm 0.3$ & $-53.4 \pm 0.4$ \\
\hline ITO/APTES & $4.1 \pm 0.5$ & $17.7 \pm 4.8$ & $0.23 \pm 0.07$ & $-48 \pm 0.8$ \\
\hline ITO/APTES/Cyt-C Ab & $9.3 \pm 0.3$ & $1.3 \pm 0.3$ & $7.0 \pm 2.0$ & $-56.6 \pm 0.1$ \\
\hline
\end{tabular}

Figure 3-7(a) and 3-7(b) compare the experimental outcomes of the equilibrium constant and Gibbs adsorption energy for the different assemblies. It is noticed that EWCRDS platform can clearly and precisely quantify the effect of each interface on the binding affinity of the Cyt-C protein. As expected and shown in Figure 3-7(a), the lowest binding affinity of Cyt-C was observed when the glass surface was functionalized with ITO/APTES (The results is shown in Figure 5, appendix 5). 


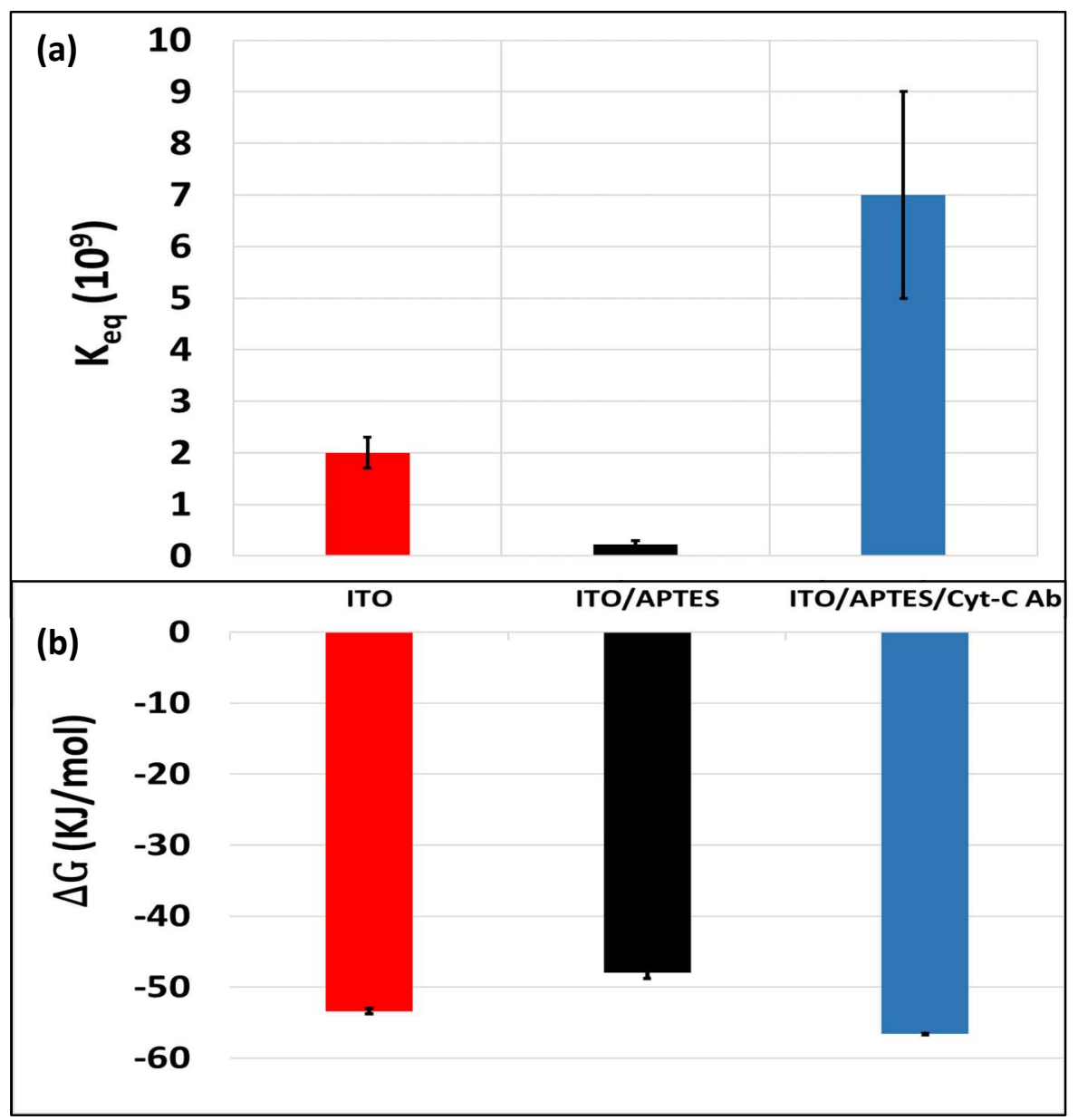

Figure 3-7: (a) Equilibrium rate constant $\left(\mathrm{k}_{\mathrm{eq}}\right)$, and (b) Gibbs free energy, for Cyt-C binding to different EW-CRDS functionalized surfaces, at open-potential condition.

Such low affinity is predictable due to the weak interaction between Cyt-C and APTES, as both APTES and Cyt-C are positively charged at a neutral $\mathrm{pH}[92,93]$. We notice a slightly increase in the $k_{e q}$ for the bare ITO, which is attributed to the favorable adsorption based on the electrostatic attraction between positively charged amino acid residues (Lysine) and the $-\mathrm{OH}$ groups of ITO. Whereas, the highest value in $k_{e q}$ was measured when the glass surface was functionalized with ITO/APTES/Cyt-C Ab (see Figure 6 in appendix 5), which indicates that the presence of a capturing Cyt-C Ab increases the binding affinity of Cyt-C. Furthermore, Figure 3-7(b) compares the Gibbs 
adsorption energy, as calculated from the $k_{e q}$ constant according to Equation 3-9, at the different assemblies functionalized on the glass surface. The negative values on the experimental data of $\Delta G$ are certainly expected from thermodynamic considerations due to the spontaneous nature of the adsorption process in this case [94], and the protein adsorption process is experimentally quantified to be highly favorable under the presence of a capture antibody on the surface of interest.

3.3.4.2. Protein Adsorption with the EW-CRDS under Electrically-Controlled Potentials

Prior to its first deployment in electrically-controlled experiments, the ITO-coated prism was electrochemically stabilized by incubating it in PBS solution for 24 hours and then applying $20 \mathrm{CV}$ cycles followed by about 20 minutes of relaxation time before any additional processing or data collection [59, 80, 95].

The effects of the electric potential on the adsorption properties of the Cyt-C protein interacting with different interfaces (ITO, ITO/APTES, and ITO/Cyt-C antibody) were measured here at constant values $(-0.2,-0.4,0.2$, and $0.4 \mathrm{~V})$ applied to the working electrode. Except for the applied potential, similar experimental procedures and data analysis already described in the OCP experiments were carried out here. 
In Figure 3-8, we show the data corresponding to the ITO interface with the measured surface coverage versus time for different concentrations of the Cyt-C protein and at different values of the applied electric potential. It is noticed that the surface coverage of Cyt-C on the ITO surface increases rapidly in the first minutes of the adsorption process and then reaches a virtual plateau regardless of the value of the applied voltage. The data quantifies the impact of the applied potential on the protein adsorption process where, beyond the transport-limited regime, a higher surface coverage is achieved for negative values of the electric potential and confirms that this particular protein adsorption process is dominated by electrostatic interactions between the positively

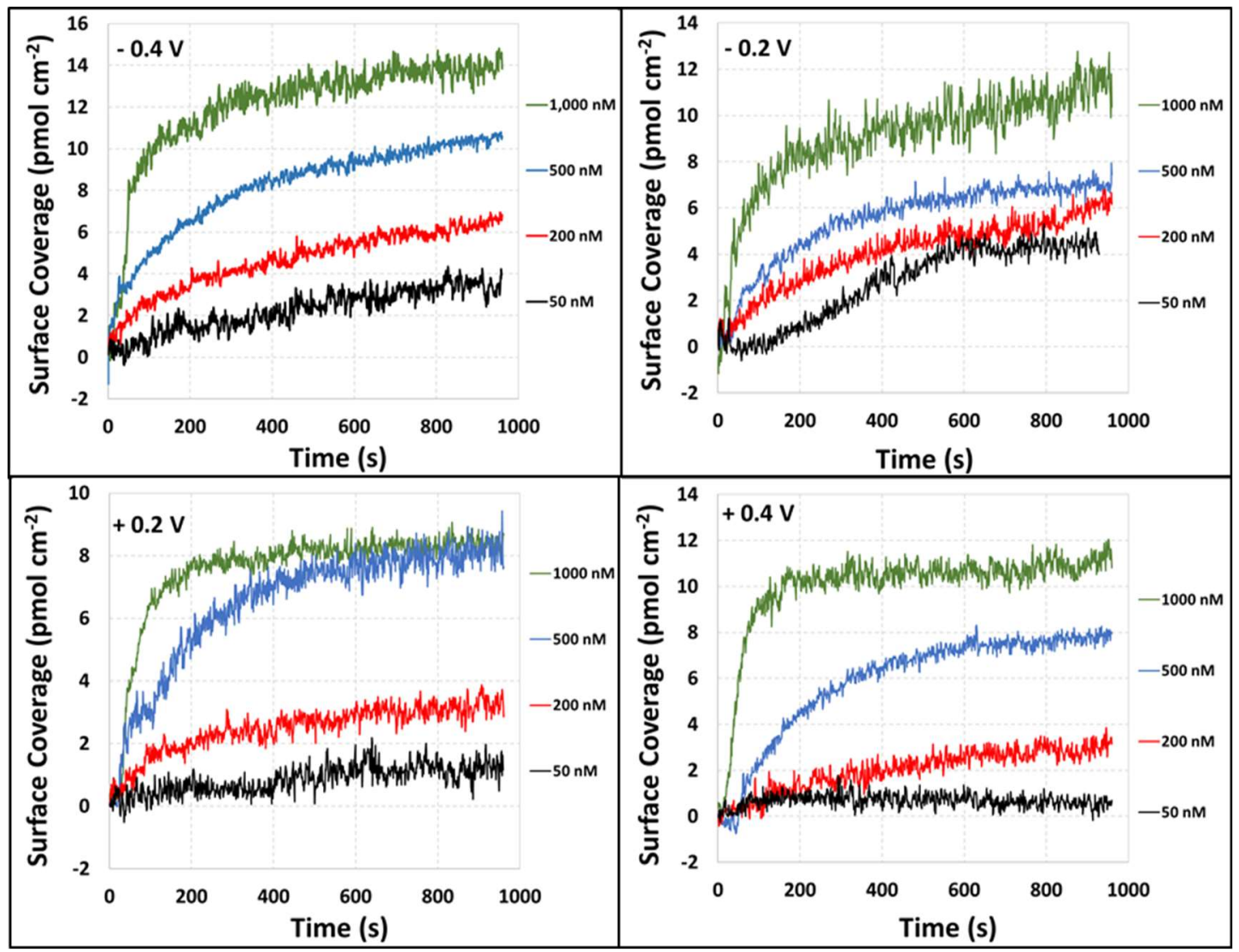


charged (lysine patch) displayed by the Cyt-C protein and the negatively charged electrode surface [96].

On the other hand, Cyt-C protein adsorption on the positive electrode can be interpreted as follows: the phosphate ions $\mathrm{HPO}_{4}{ }^{2-}$ will aggregate to form a cluster structure with a specific arrangement, which functions as a bridge between the positively charged lysine residues of Cyt-C protein and positively charged electrode [96].

Table 3-2: The Equilibrium rate Constant $\left(k_{e q}\right)$ for Cyt-C Binding to different EW-

CRDS Functionalized Surfaces with different values of external electric potential.

\begin{tabular}{|l|c|c|c|c|}
\hline $\begin{array}{l}\text { EW-CRD } \\
\text { functionalized surface }\end{array}$ & \multicolumn{4}{|c|}{$k_{\text {eq }}\left(\mathbf{1 0}^{\mathbf{9}}\right)$} \\
\hline & $\mathbf{- 0 . 4 ~ V}$ & $\mathbf{- 0 . 2 ~ V}$ & $\mathbf{0 . 2 ~ V}$ & $\mathbf{0 . 4} \mathbf{~}$ \\
\hline ITO & $3.10 \pm 0.41$ & $2.43 \pm 0.39$ & $0.96 \pm 0.21$ & $0.62 \pm 0.18$ \\
\hline ITO/APTES & $0.11 \pm 0.12$ & $0.20 \pm 0.18$ & $0.26 \pm 0.23$ & $0.28 \pm 0.19$ \\
\hline ITO/APTES/Cyt-C Ab & $11.05 \pm 0.38$ & $8.15 \pm 0.43$ & $1.83 \pm 0.30$ & $0.67 \pm 0.28$ \\
\hline
\end{tabular}

Similar data were collected and analyzed (see appendix 6 ) for the other interfaces (ITO/APTES and ITO/Cyt-C antibody). However, in Table 3-2 we summarize the experimental results for all of the interfaces on the adsorption equilibrium constant of Cyt$\mathrm{C}$ under various applied potential. 
In addition, these results for the adsorption equilibrium constant and the corresponding adsorption Gibbs energy are shown in Figure 3-9. As Figure 3-9(a) reports, when the electrode surface becomes negatively charged, the adsorption of Cyt-C proteins increase only slightly on the ITO interface. However, it increases significantly when the glass surface is fully functionalized with the ITO/APTES/Cyt-C Ab assembly, which quantifies the high binding affinity due to the Cyt-C antibody and the strong electrostatic interaction between the positively charged amino acid residues (Lysine) and negatively

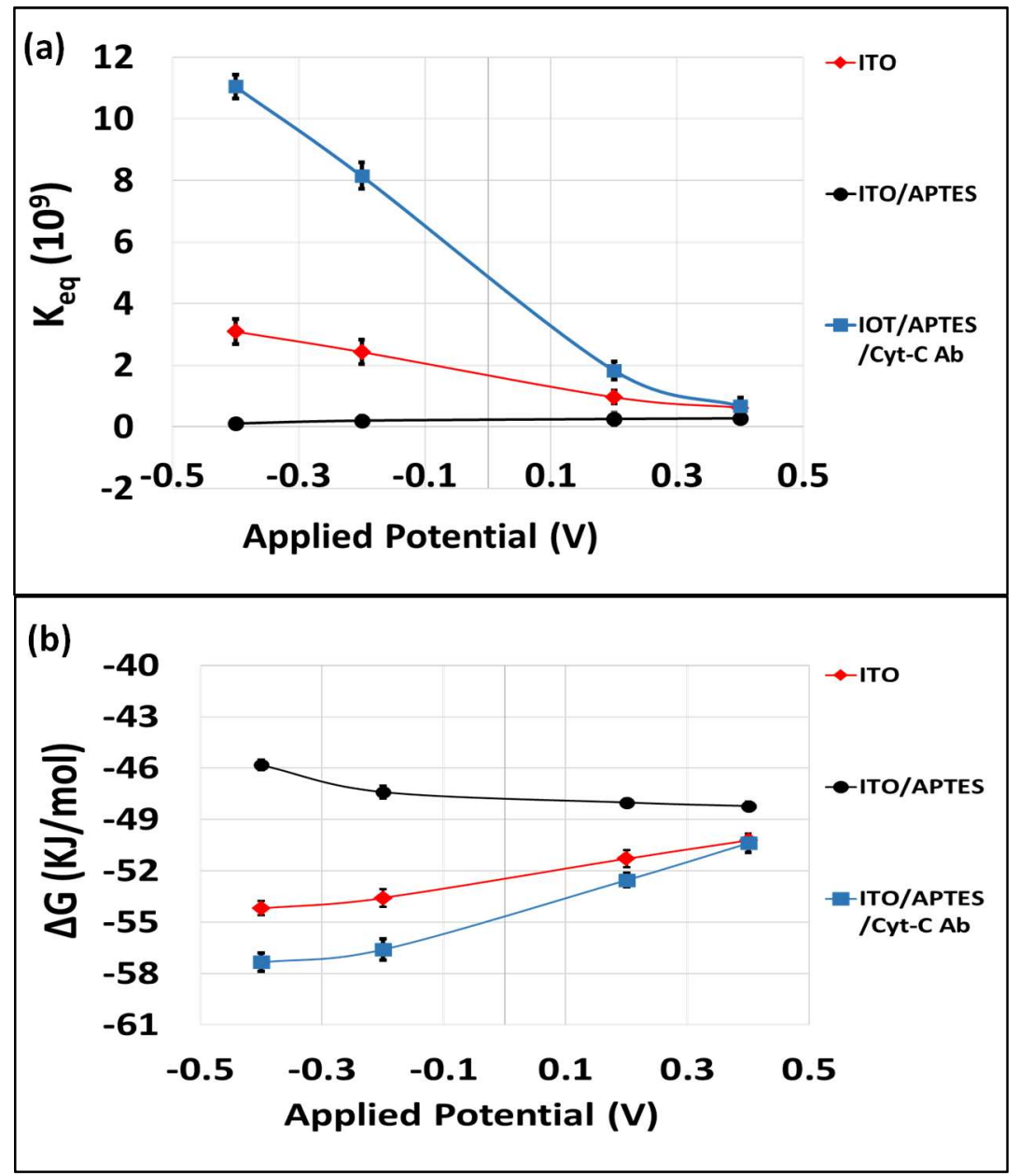

Figure 3-9: (a) The values of adsorption equilibrium constant, and (b) Gibbs free energy, versus applied electric potential $(-0.4,-0.2,0.2$, and $0.4 \mathrm{~V})$ for Cyt-C binding to different EW-CRDS functionalized surfaces. 
charged electrode. In contrast, there is no significant change in the adsorption rate due to the weak interaction between Cyt-C and the ITO/APTES surface.

As shown in Figure 3-9 (b), the minimum values of the Gibbs adsorption energy occur when the applied potential becomes negatively charged, especially for the ITO/APTES/Cyt-C Ab interface. The EW-CRDS platform allows us to precisely quantify those phenomena that certainly can be understood under a rationale of first principles but that sometimes occurs at challenging or inaccessible experimental configurations.

\subsubsection{Cyclic Voltammetry with the Electro-Active EW-CRDS Platform}

Optical absorbance measurements were performed using ring-down time data in the EW-CRDS platform under cyclic voltammetry (CV) potential modulation. The Cyt-C protein interaction with the three different assemblies (ITO, ITO/APTES, and ITO/Cyt-C antibody) immobilized on the internal reflection surface of the glass prism was probed under $\mathrm{CV}$ scan. The $\mathrm{CV}$ scan was set with an electric potential ranging from $-0.3 \mathrm{~V}$ to +0.6 $\mathrm{V}$ and a scan rate of $30 \mathrm{mV} / \mathrm{s}$. First, a background optical signal was acquired without the presence of the Cyt-C proteins in the flowcell, (which at this time was filled with just PBS solution). Optical data of ring down time under CV scanning was synchronized and collected using our LabView routine. After this data acquisition, $300 \mu \mathrm{L}$ of $500 \mathrm{nM} \mathrm{Cyt-C}$ solution were injected into the flowcell and were allowed to incubate for 15 minutes to stabilize the adsorption process of Cyt-C proteins on top of the functionalized surface. Subsequently, the flowcell was rinsed with $900 \mu \mathrm{L}$ of PBS solution to remove unbound species. Next, optical data under identical CV scanning conditions was acquired to interrogate the Cyt-C effects on the analytical interface. As described in Equation 3-1, 
measurements of the optical data of cavity ring-down times in both the absence and presence of Cyt-C allowed us to generate the optical absorbance. In Figure 3-10, the optical absorbance of Cyt-C is plotted against the applied electric potential for each functionalizing assembly on the glass surface of EW-CRDS prism; please notice that several CV cycles are folded into the same plot.

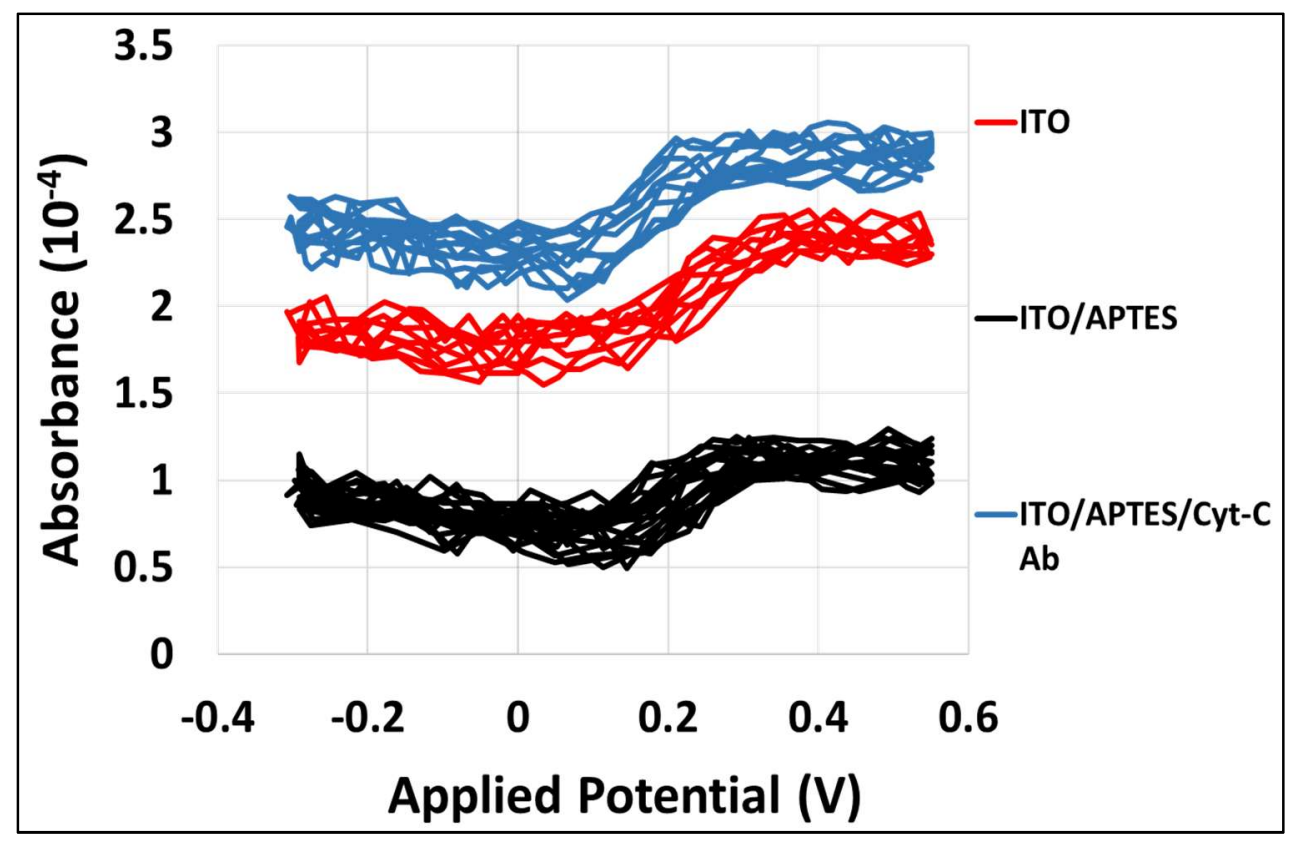

Figure 3-10: Absorbance of Cyt-C plotted against electric potential at each functionalized surface of the EW-CRDS interface. The bulk concentration of Cyt-C protein was kept constant at $500 \mathrm{nM}$ and the $\mathrm{CV}$ potential modulation parameters where all the same: range of $-0.3 \mathrm{~V}$ to $+0.6 \mathrm{~V}$.

For measurements at each interface (ITO, ITO/APTES, and ITO/Cyt-C Ab), the bulk concentration of the Cyt-C protein solution was kept at $500 \mathrm{nM}$. Between each set of measurements displayed in Figure 3-10, the sensing surface of the prism was re-cleaned using the protocols described in section 3.3.2.3. 
The Cyt-C molecules when dispersed in the bulk PBS solution at the 7.2-pH are known to be in the oxidized state. Therefore, at positive values of the applied potential on the surface of the working electrode in Figure 3-10, the surface-adsorbed Cyt-C species are expected to be fully oxidized. The surface density $\left(\Gamma_{t o t}=\Gamma_{0 x}\right)$ of the adsorbed species can then be calculated using Equation 3-2 from absorbance data at positive values of the applied potential; the surface density results for all measured assemblies are summarized in Figure 3-11(a). By considering that a full monolayer of Cyt-C protein corresponds to about $22 \mathrm{pmol} \mathrm{cm}$, we obtain the following portions of a full monolayer for each assembly: $28 \%$ for ITO, $9 \%$ for ITO/APTES, and $34 \%$ for ITO/APTES/Cyt-C-Ab. As expected, Figure 3-11(a) confirms that the functionalizing assembly has an important role on the Cyt-C adsorption process; the presence of the Cyt-C capturing antibody greatly enhances the binding affinity and adsorption process, which is consistent with data from our previous sections.

In Figure 3-10, the optical absorbance data under CV scans shows a clear redox transition for all the measured assemblies. As the applied potential is driven towards negative values, the protein is expected to undergo a reduction process, and a drop in absorbance values confirms this process. However, in principle we may have a mixture of reduced and oxidized species adsorbed to the surface under investigation. Equation 3-6 was used to calculate the fraction of Cyt-C species that are converted to the reduced state (from the original oxidized state). Figure 3-11(b) summarizes the results for the fraction of the overall population of adsorbed protein species, $\Gamma_{\text {red }} / \Gamma_{\text {tot }}$, that undergoes redox transition during CV scanning process. As one can observe from the data displayed in Figure 3-11(b), 
the redox transition of the Cyt- $\mathrm{C}$ protein decreases for each additional layer that is added to the assembly functionalizing the glass surface of the EW-CRDS platform.

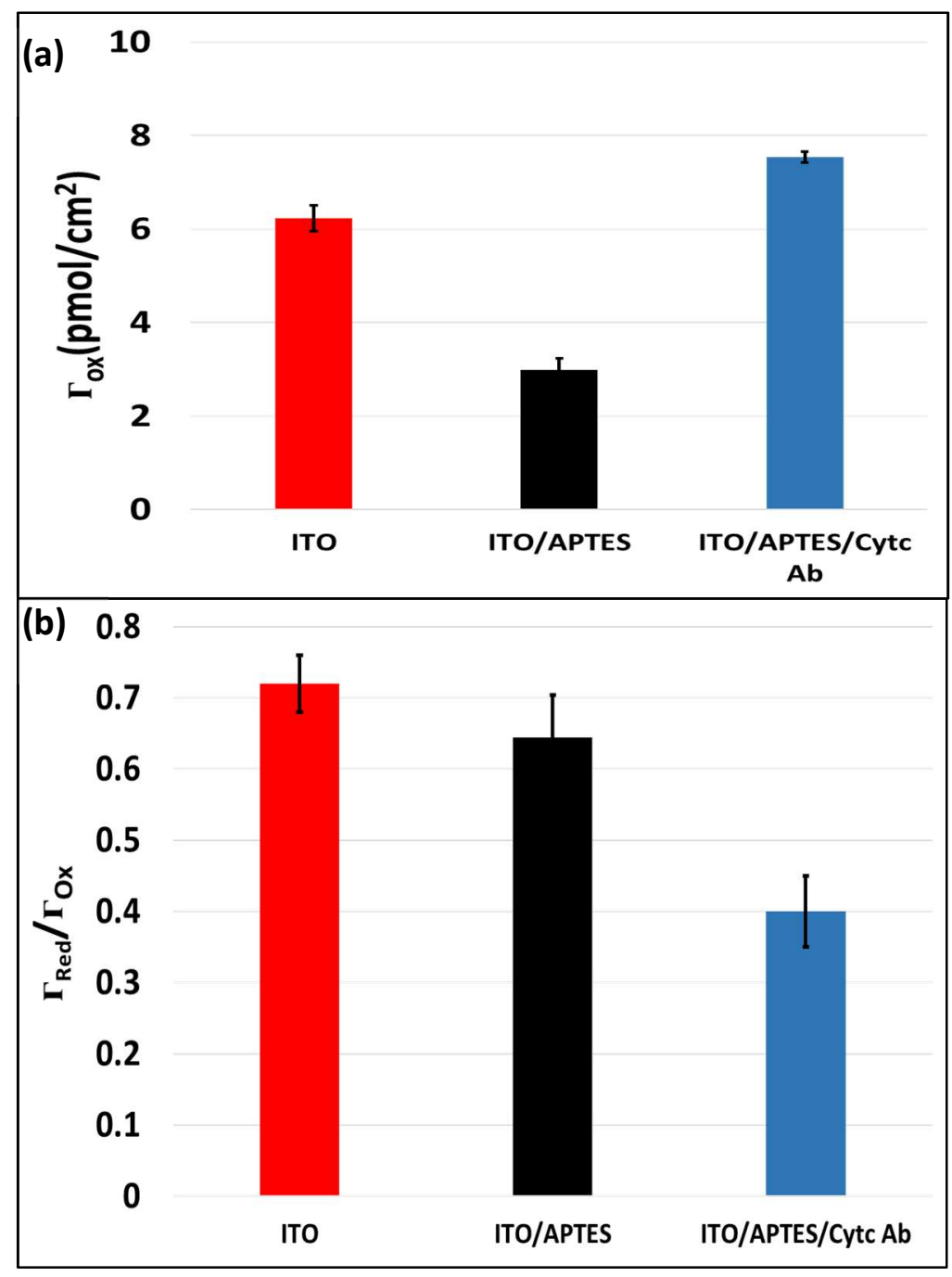

Figure 3-11: (a) The measured total surface coverage density of Cyt-C in its oxidized form at different interfaces. (b) The fraction for each interface of surface density of reduced species over the total surface density in the oxidized form.

More importantly, the developed EW-CRDS platform is able to clearly access and quantify the effects on the redox transition as individual molecular layers are added to the 
interface under optical interrogation, even when the surface density of redox species are at low submonolayer levels.

\subsection{Conclusions}

An electrically-active cavity-ring-down spectroscopic technique that is able to perform spectroelectrochemical investigations of redox events at solid/liquid interfaces by using the evanescent field of a cavity-confined light pulse was described. A high-quality, ultra-thin, transparent conductive electrode film of indium tin oxide (ITO) was coated on the interface of total internal reflection of the EW-CRDS platform to deliver a ring-down time of about $900 \mathrm{~ns}$, which enabled us to perform spectroelectrochemical studies at solid/liquid interfaces.

Some capabilities of the developed platform were demonstrated here by addressing the impact of applied surface potential on adsorption kinetics processes of a Cyt-C protein into a multilayer assembly. The results indicated the high dependence of the protein adsorption on the nature and electric potential of the adsorbent surface. The results of equilibrium rate constant and Gibbs energy for adsorption shows that the ITO/APTES/Cyc$\mathrm{C} \mathrm{Ab}$ combination increases the binding affinity of Cyt-C protein in the OCP experiment. Moreover, the adsorption of Cyt-C protein can be controlled by applying an external potential to the adsorbent surface. The results indicate a high association rate when the negative values of electric potential were applied, which is attributed to the strong electrostatic interaction. 
The signal from the EW-CRDS platform was used to optically follow the timedependent faradaic current originated from a submonolayer protein layer undergoing redox exchanges driven by a CV modulation. We show here that EW-CRDS provides strong analytical signals that can be readily monitored even for small surface-densities of species involved in the redox process. The results for a synchronized EW-CRDS measuremed with the CV scan show the ability of the EW-CRDS platform as a powerful tool to investigate the redox reactions of the adsorbed electro-active species, at the modified electrode, even at very small surface-densities.

The studies herein demonstrate that the EW-CRDS platform is a powerful and straightforward technique to probe interfacial phenomena and a wide range of applications that could be investigated such as biosensors, and bioanalytical systems. 


\section{CHAPTER 4}

\section{REAL TIME INFLUENZA VIRUS DETECTION WITH ELECTRO-CHEMICAL EW- CRDS PLATFORM}

\subsection{Introduction}

The ability of the electro-active EW-CRDS platform to measure electrode-driven redox events and adsorption kinetics of a redox probe onto multilayer immunoassay assembly at a single diffraction-limited spot has been demonstrated and confirmed in Chapter 3.

Although the electrochemical immunosensoring techniques have been extensively used for the detection of analytes with small volume and at reasonable costs [97-99], they still lack sensitivity to probe redox species with weakly absorbing chromophores and/or at low surface concentrations. Furthermore, the undesired background signal at low surface densities that is produced by non-faradaic components from bulk species, is typically problematic. To overcome such hurdles, the alternative is spectroelectrochemical techniques $[47,49,88,100,101]$, in which a probing light source is spectrally tuned to interrogate a transition of the optical signal associated with the electrochemically driven electron-transfer process of the surface-adsorbed redox molecules; this plays a crucial role in avoiding non-faradaic components that typically impede conventional electrochemical 
approaches using electrical signals a lone.

Due to the selectivity and the high sensitivity of EW-CRDS device to probe weakly absorbing redox species at low surface concentrations, it may prove to be a valuable tool in label-free biosensing. This chapter describes the development of a direct immunosensor based on a sandwich immunoassay approach for the hemagglutinin (HA) protein for the influenza A virus $\mathrm{H} 5 \mathrm{~N} 1$ detection using the EW-CRDS platform. First, the change in the optical signal was monitored in real-time during the construction of an immunoassay assembly (primary Ab/virus antigen/Ab-labeled redox probe) onto a transparent electrode. CV potential scans under synchronous optical readout were employed, where the electron transfer between a redox probe and working electrode during the potential modulation is recognized by the optical signals, which serve as a fingerprint for the sensing methodology.

\subsection{Principle of Bio-sensing Using EW-CRDS Platform}

The biosensing strategy using EW-CRDS is performed by the modification of the surface in multiple stages. The principle of EW-CRDS biosensor is based on a sandwich immunoassay approach as illustrated schematically in Figure 4-1. The fabrication of biosensors mainly involves three steps: In the first step, the immobilization of the capture $\mathrm{Ab}$ (receptor $\mathrm{Ab}$ ) on the surface of EW-CRDS was achieved by forming a monolayer of $\mathrm{a}$ specific chemical group (as APTES) or using a connecting element between the capture antibody and electro-active surface. Once the target analyte is introduced, it will bind specifically to the capture $\mathrm{Ab}$, which promotes the binding of a secondary $\mathrm{Ab}$ that has been labeled with an electrochemically active reporter that will act as a transduction element for 
monitoring molecular binding events, and can be electrochemically modulated on the EWCRDS platform to provide the probing optical signal.

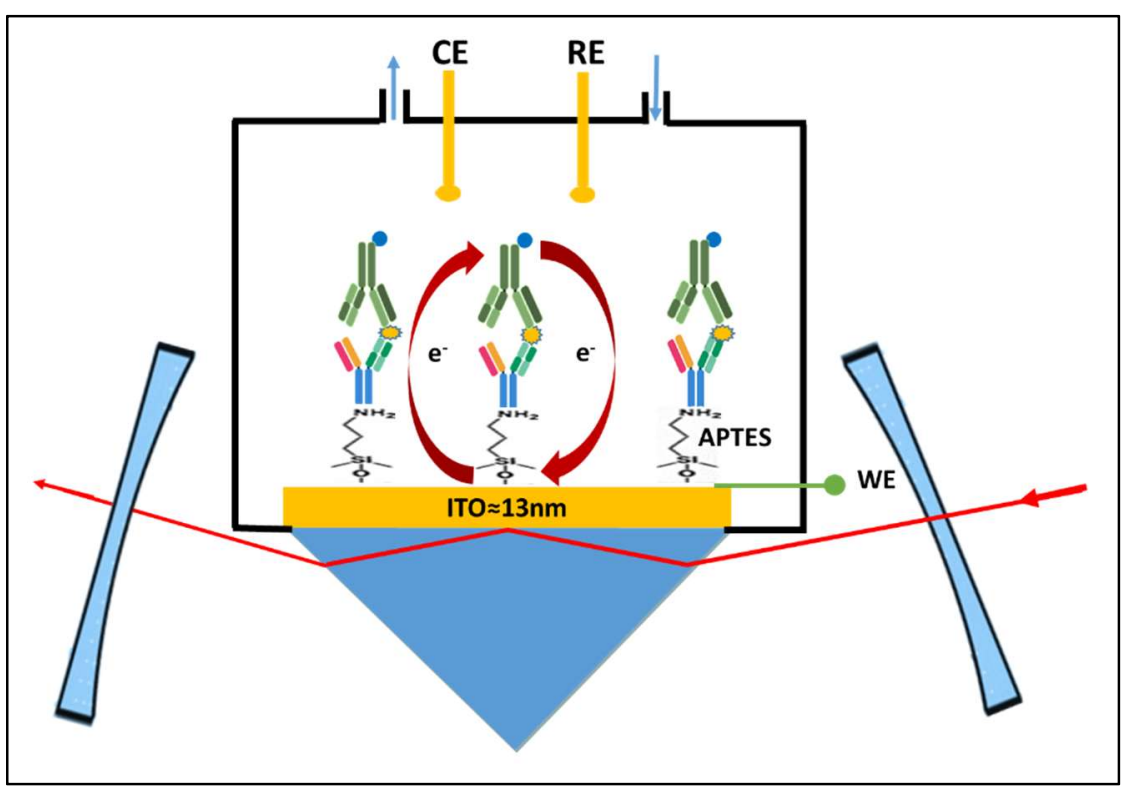

Figure 4-1: Schematic illustration of a sandwich bioassay strategy using electro-active EWCRDS platform. The strategy of the fabrication of the biosensor begins with the functionalization of the surface with the APTES chemical group to attach the capture Ab to the surface, once the target analyte is bound to the sensing interface, it promotes the binding of a secondary $\mathrm{Ab}$ that has been labeled with a redox-active optical reporter.

\subsection{Experiment}

\subsubsection{Experimental setup}

The experimental setup of EW-CRDS and the electro-active flowcell are identical to that described previously in Section 3.3.1. 


\subsubsection{Chemicals and reagents}

Phosphate buffered saline (PBS) $50 \mathrm{mM}(\mathrm{pH}=7.4)$ and Methylene blue ester $(5 \mathrm{mg}$, molecular weight $=598.12 \mathrm{~g} / \mathrm{mol}$ ) were purchased from Biosearch Technologies (Novato, California). (3-Aminopropyl) triethoxysilane (APTES) was purchased from Sigma Aldrich. Bovine Serum Albumin (BSA)(purchased from Sigma Aldrich), A monoclonal anti-H5 (H5N1) capture antibody $(250 \mu \mathrm{g}, 2 \mathrm{mg} / \mathrm{ml})$, polyclonal anti-H5 (H5N1) secondary antibody $(10 \mu \mathrm{g} / \mathrm{ml}, 1 \mathrm{ml}$, molecular weight $=144 \mathrm{kDa}$, goat polyclonal $\mathrm{IgG})$, and recombinant influenza A hemagglutinin (HA) protein of the $\mathrm{H} 5 \mathrm{~N} 1$ influenza virus (200 $\mu \mathrm{g} / \mathrm{ml}, 0.5 \mathrm{ml}$ ) were purchased from Santa Cruz Biotechnologies, and diluted in PBS buffer to different concentrations $20,50,100$, and $200 \mathrm{ng} / \mathrm{ml}$.

\subsubsection{Labeling of Secondary Antibody with a Redox Probe}

The conjugation of secondary $\mathrm{Ab}$ with a redox active probe is essential to form an electro-optical signal response of EW-CRDS for sandwich immunoassay based detection. Methylene blue (MB) is a model organic redox indicator that belongs to the phenothiazine family, has a formal potential in the range of 0.10 to $0.40 \mathrm{~V}$ in $\mathrm{pH} 4-11$, and has been widely used as an electron transfer mediator $[92,102]$. In recent years, MB has been used in DNA hybridization detection and aptamer-based biosensor $[102,103]$. The spectroelectrochemical properties have been investigated [100, 104], and as Figure 4-2 describes, the blue colored oxidized form of MB can be reduced using electrochemical potential modulation to colorless Leuco-Methylene Blue (LMB). MB is an autoxidizing

dye; meaning that at low concentrations, it forms in equilibrium with LMB a reversible redox-couple. 
The conjugation of the secondary $\mathrm{Ab}$ with a redox probe $\mathrm{MB}$ was done by our group. The MB derivative NHS-ester, which has the same reduction properties as MB, was purchased from Biosearch Technologies (MB-1000S-5, $5 \mathrm{mg}$, molecular weight $=598.12$ $\mathrm{g} / \mathrm{mol}$ ). Such material has a carboxylic acid group that promotes the direct coupling to amino groups of antibodies.

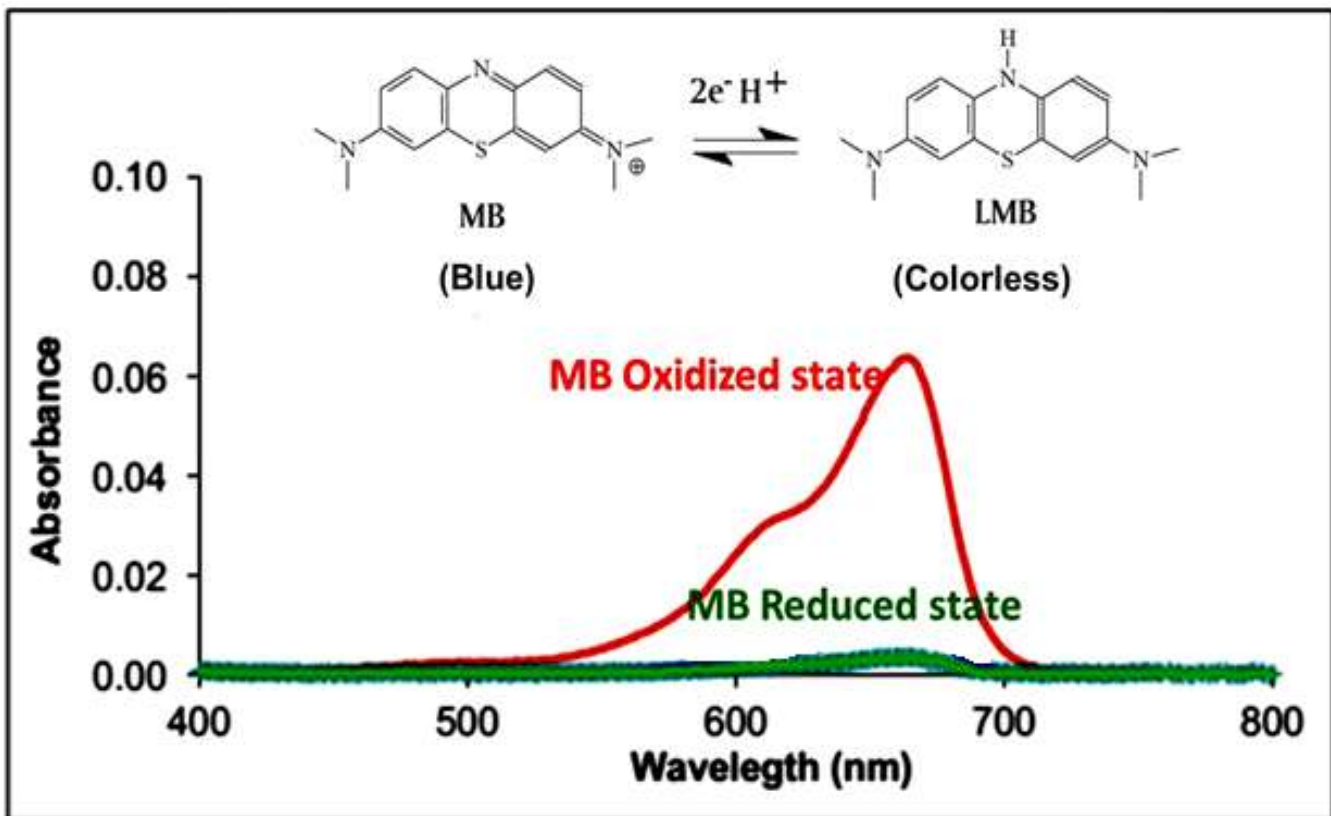

Figure 4-2: UV Vis spectra of oxidized (red curve) and reduced form (green curve) of MB.

MB was reduced chemically by using ascorbic acid $22 \mathrm{mM}$ in the presence of $500 \mathrm{mM}$ $\mathrm{HCl}$.

The conjugation began by mixing a secondary polyclonal Ab for the influenza $\mathrm{A}$ H5N1 with MB at room temperature for 2 hours, in a labeling kit (A10235, Thermo Fisher Scientific, Waltham, Massachusetts). In the last step, the conjugated polyclonal anti-H5 (H5N1) was purified using a resin column (Thermo Fisher Scientific, Waltham, Massachusetts) and a $50 \mathrm{mM}$ PBS solution as elution buffer. Then, the concentration of MB-labeled $\mathrm{Ab}$ was determined by using UV-Vis absorption spectroscopy (details in 
Appendix 7). As we can observe in Figure 4-3, the shift in the absorption spectra between free MB (blue curve) and secondary H5N1 Ab (red curve), indicates the successful labeling procedure of the secondary $\mathrm{Ab}$ with the MB dye.

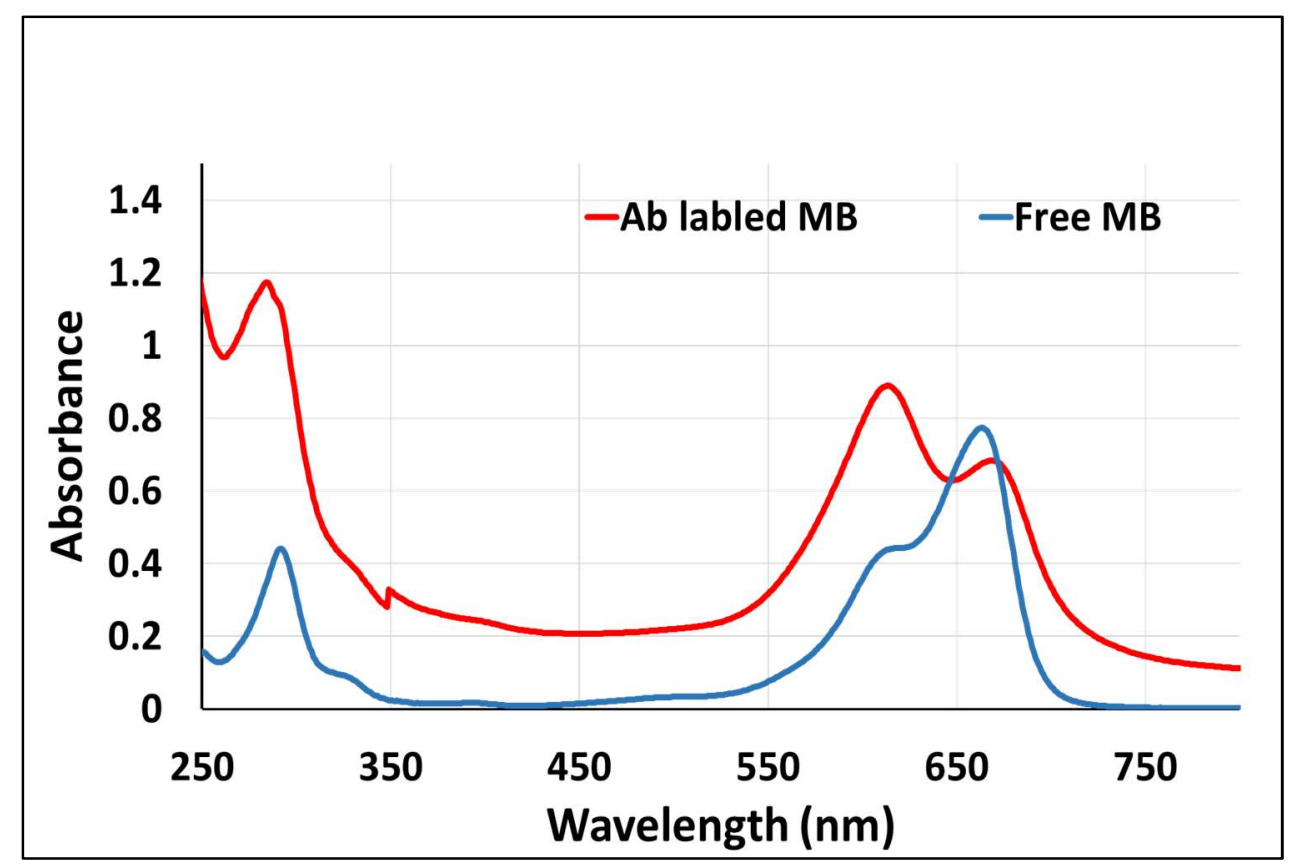

Figure 4-3: UV Vis absorption spectra of free MB ((blue curve) and secondary H5N1 $\mathrm{Ab}$ (red curve), the shift in the two spectra is evidence of successfully aimed conjugation.

\subsubsection{Sensor Functionalization Methods}

The sensor surface of the ITO-coated prism was cleaned to remove any contamination by sequentially sonicating the surface of ITO with deionized (DI) water, acetone, and ethanol for 15 min each time, and then dried using $\mathrm{N}_{2}$ gas. The prism was mounted into the flow cell. The surface of the ITO-coated prism within the sensing area was in situ functionalized with APTES by injecting a solution containing $100 \mu 1$ of APTES 
and 4 to 5 drops of DI water in $10 \mathrm{ml}$ ethanol for $6 \mathrm{~h}$. After several washing cycles with ethanol and DI water, the result was an amino-propyl coating with active amino-group sites. The cell was partially filled with $200 \mu$ of a PBS solution of concentration $50 \mathrm{mM}$ $(\mathrm{pH}=7.4)$. A manually activated syringe pump was used for all liquid injection into the electrochemical cell. Between each experiment, the EW-CRDS device was re-cleaned using the protocols described in section 3.3.2.3.

\subsection{Results and Discussions}

\subsubsection{Real-Time Influenza Virus Immunosensor with EW-CRDS}

The procedures of building up a sandwich immunoassay for the detection of HA influenza virus antigen (H1N5) using the EW-CRDS platform were monitored by recording the ring-down time as a function of time, as shown in Figure 4-4.

At time 'a', the ITO/APTES surface was functionalized with capture Ab species by injecting $200 \mu \mathrm{l}$ of monoclonal antibody-H5 (H5N1) at a concentration of $2 \mu \mathrm{g} / \mathrm{ml}$. After approximately $3 \mathrm{~h}$ the equilibrium concentration was achieved, which caused $\tau$ to reduce slowly from 0.838 to $0.785 \mu$ s. In order to remove any unbounded antibodies, the flow cell was washed gently (at time '1') with $200 \mu$ l of PBS buffer, and achieved equilibrium concentration after approximately 15 min which caused $\tau$ increase to about $0.800 \mu$ s.

At the time indicated by 'I', $100 \mu \mathrm{g} / \mathrm{ml}$ of BSA solution was introduced to block nonspecific adsorption sites. This produced only a slight change of $\tau$ from 0.800 to 0.793 $\mu$ s, after approximately $30 \mathrm{~min}$ the flow cell was rinsed gently again (at time ' 2 ') with PBS 
to remove unbound BSA molecules this recovered $\tau$ near the value before BSA injection (0.798 $\mu \mathrm{s})$.

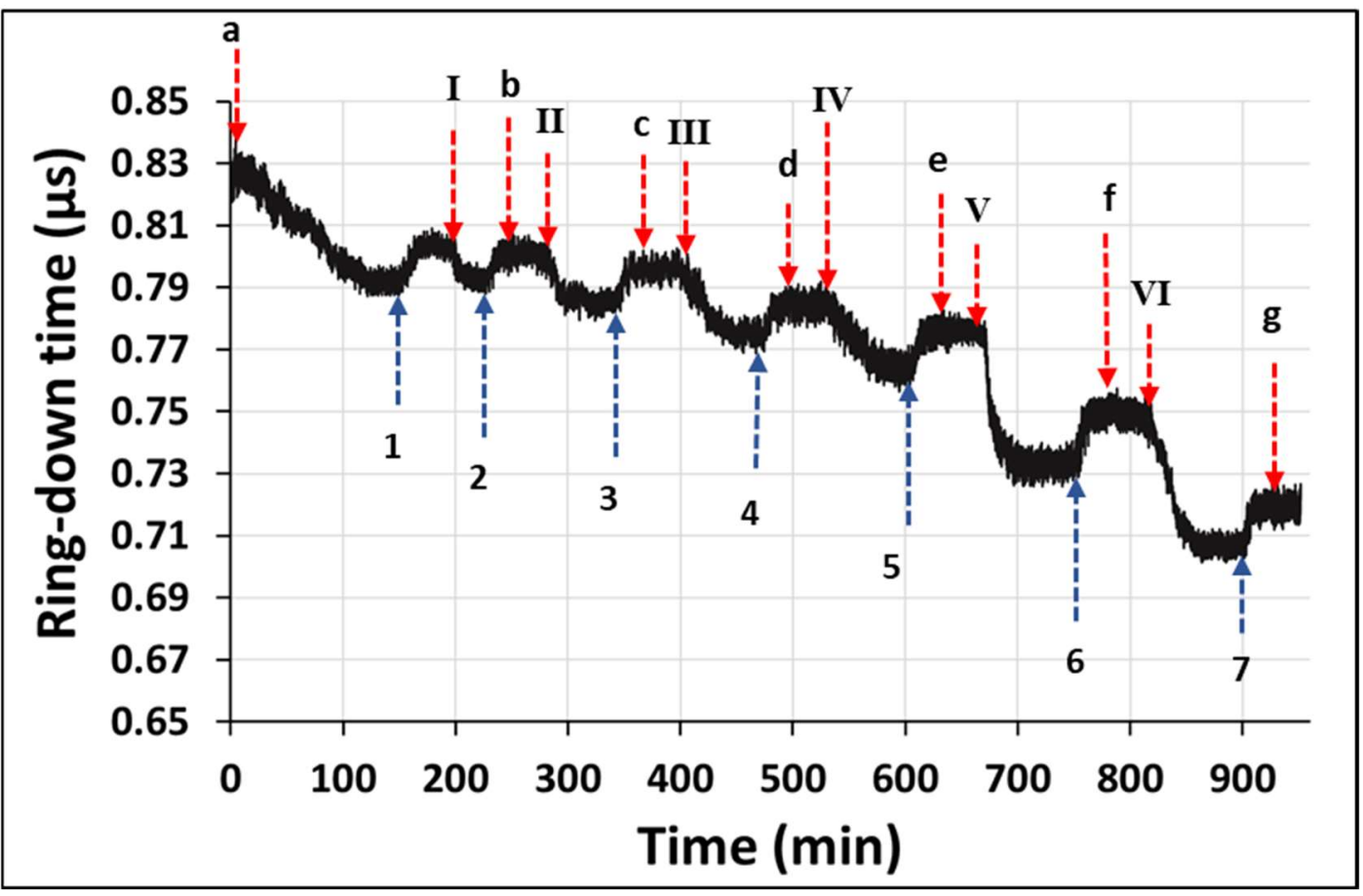

Figure 4-4: Influenza virus immunosensor measured on EW-CRDS platform. The ringdown time recorded as a function of time during the Steps of the sandwich immunoassay assembly (see text and Table 2).At time 'a' the capture Ab was introduce to the flow cell, at time 'I' BSA was added, at the times indicated by 'b', 'c', 'd', 'e', and 'f' virus antigen was added with concentrations $0,20,50,100$, and $200 \mathrm{ng} / \mathrm{ml}$, respectively. The numbers indicated by 'II', 'IV', 'V', and 'VI' are the times when the MB-labeled Ab were added after each antigen concentration. The number indicated by '1', '2', '3', '4', '5', '6', and '7' are the times when the flow cell was rinsed with $200 \mu \mathrm{l}$ of PBS.

In the absence of the HA virus antigen ( $0 \mathrm{ng} / \mathrm{ml}$, the time indicated by ' $b$ '), at the time indicated by 'II', $200 \mu \mathrm{l}$ of MB-labeled polyclonal secondary Ab solution with concentration $10 \mu \mathrm{g} / \mathrm{ml}$ was injected. After $1 \mathrm{~h}$ the flow cell was rinsed with PBS, and we 
observed that $\tau$ increased fairly rapidly before it reached the equilibrium value $(0.796 \mu \mathrm{s})$, which is near what was previously obtained, after $15 \mathrm{~min}$. This can be explained according to Figure 4-5(a) that, in the absence of the virus antigen, the probe species were not bound to the surface and the optical signal response vanished after the flow-cell was rinsed (at time ' 3 '). This indicates that the BSA worked as expected to eliminate nonspecific adsorption events. At time ' $\mathrm{c}$ ' the lowest concentration of virus antigen $(20 \mathrm{ng} / \mathrm{ml})$ was

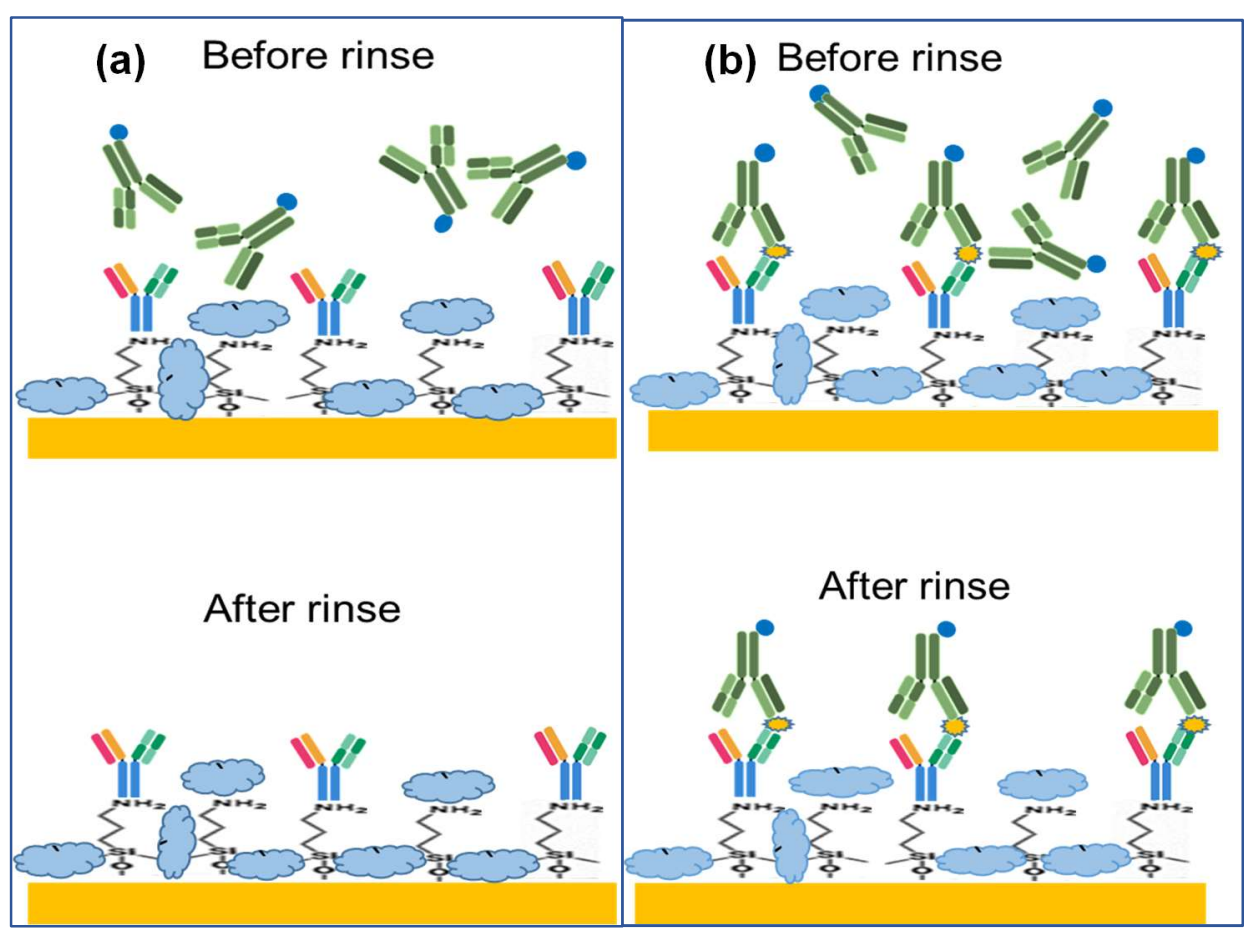

Figure 4-5: Schematic representation of the results in the Figures 4-4 in case (a) When the virus antigen is absent (b) In the presence of the antigen, MB-labeled H5N1 Ab is adsorbed to the surface. The BSA worked to block the nonspecific adsorption sites. introduced and allowed to bind to the surface bound capture antibodies for 30 min before 
being rinsed again with PBS (at time '4'). We observed no change in $\tau$ due to introduction of the virus antigen.

In the next step we note that, at time 'III', the addition of more secondary Ab results in further reduction of the photo lifetime, as after approximately $1 \mathrm{~h}$ the adsorption process reached the plateau region, after which the flow cell was rinsed with PBS buffer to remove unbound antibodies. After approximately $15 \mathrm{~min}$ the equilibrium concentration was achieved, and the value of $\tau$ was $0.785 \mu$ s. In contrast, Figure 4-5(b) illustrates the results in Figure 4-4, where the presence of an HA virus antigen increased the binding affinity of secondary $\mathrm{Ab}$ to the surface and provided a strong analytical response even after rinsing the flow-cell.

The same procedures were repeated for other concentrations of virus antigen (50, 100 , and $200 \mathrm{ng} / \mathrm{ml}$ ) at times 'd', 'e', and 'f' respectively. More MB-conjugated secondary Ab was added at the times indicated by 'IV', 'V', and 'VI' respectively, and more washing processes were completed at the times indicated by ' 5 ', ' 6 ', and ' 7 ' respectively were done to remove unbound species. The average ring-down times after each washing process of

Table 4-1: The data of average ring-down times obtained after each washing process of MBE-conjugated Ab in Figure 4-4.

\begin{tabular}{|c|c|c|c|c|c|}
\hline Time in Fig.4-4 & c & d & e & f & g \\
\hline $\begin{array}{c}\text { Virus } \\
\text { conc.(ng/ml) }\end{array}$ & 0 & 20 & 50 & 100 & 200 \\
\hline$\tau(\mu \mathbf{s})$ & $0.796 \pm 0.002$ & $0.785 \pm 0.003$ & $0.776 \pm 0.002$ & $0.750 \pm 0.002$ & $0.719 \pm 0.002$ \\
\hline
\end{tabular}


the flow cell with PBS buffer, for each virus antigen concentration, and after adding MBconjugated secondary Ab are recorded in Table 4-1.

The absorbance values were calculated using the Equation 3-1, where $\tau_{0}$ and $\tau$ are the average ring-down time for the background (which corresponds to average ring-down time at time ' $b$ ' in Figure 4-4) and for each virus antigen concentration in Table 4-1, respectively.

-To determine the limit of detection, the optical absorbance was plotted for different bulk concentrations of the virus antigen solution, as shown in Figure 4-6, and the calibration curve confirms the linear relationship between measured absorbance and concentration. The limit of detection was determined using a 3-sigma standard, which

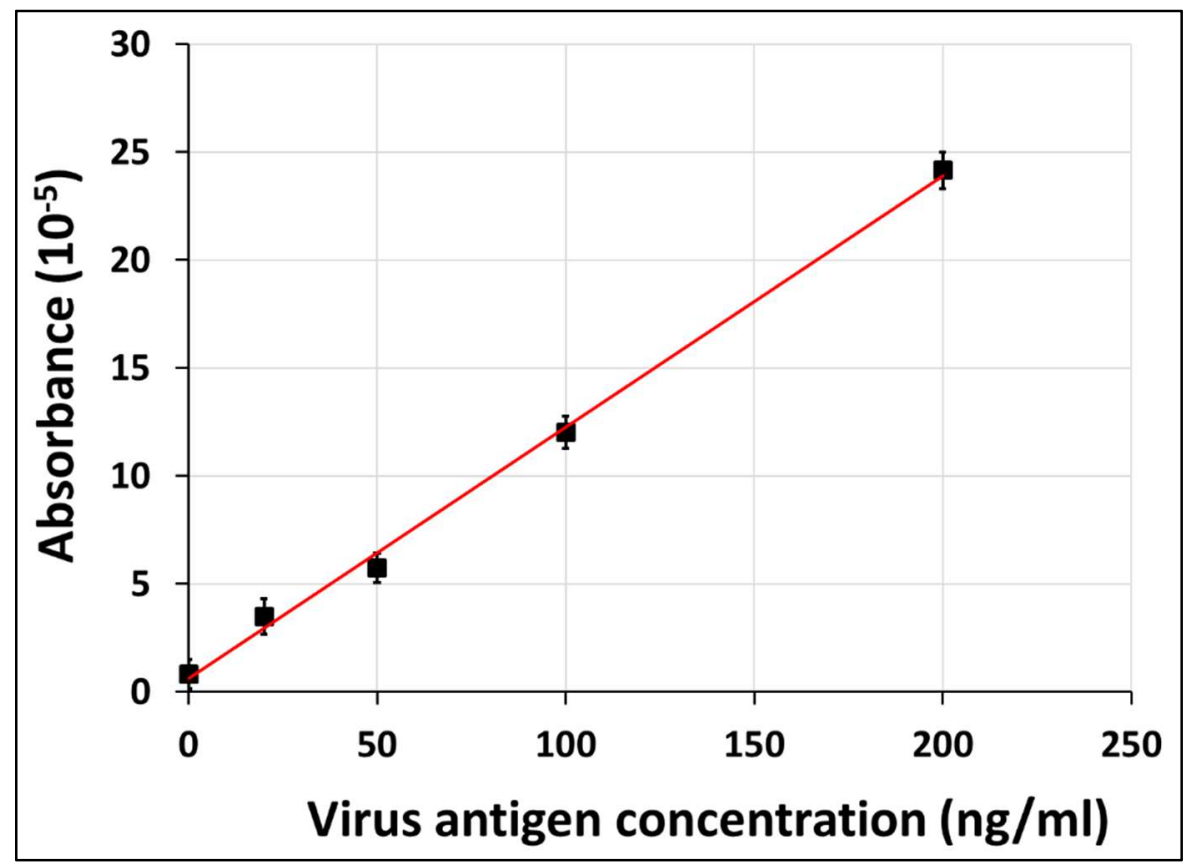

Figure 4-6: The calibration curve of the optical absorbance measured by EW-CRDS for each concentration of virus antigen, which allows determining the limit of detection. 
corresponds to an analyte concentration that would generate a signal that is three-fold the signal measured when no virus analyte was present in the system. This procedure resulted in a detection limit of the order of $15 \mathrm{ng} / \mathrm{ml}$ or $280 \mathrm{pM}$.

\subsubsection{Cyclic Voltammetry (CV)}

The optical absorbance data for the sandwich immunoassay were collected with the EW-CRDS platform under CV potential modulation. Optical data of ring down time under CV scanning was synchronized and collected using our Labview routine. The baseline

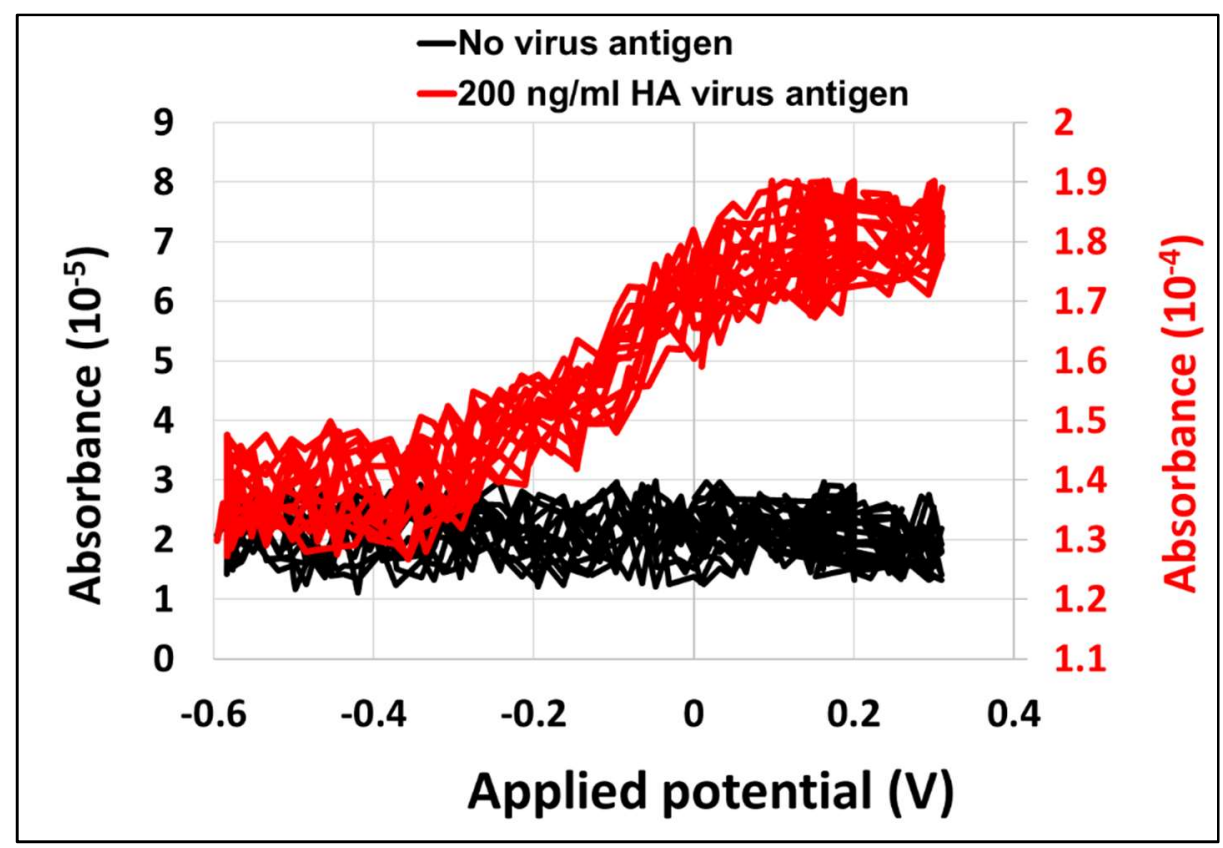

Figure 4-7: The optical signal response at $532 \mathrm{~nm}$ as measured by the EW-CRDS platform under $\mathrm{CV}$ potential modulation. Where the red trace represents the optical absorbance in the presence of HA virus antigen. The black trace when the virus antigen is not present and the probe species are not bound to the surface; the analytical signal vanishes after the flow-cell was rinsed. 
optical signal was collected after adsorption of the influenza virus antigen, while the reporter optical signal was collected after incubation of the MB-labeled $\mathrm{H} 5 \mathrm{~N} 1 \mathrm{Ab}$ at a concentration of $10 \mu \mathrm{g} / \mathrm{ml}$. The CV scan was set with an electric potential ranging from 0.6 $\mathrm{V}$ to $+0.3 \mathrm{~V}$ and a scan rate of $30 \mathrm{mV} / \mathrm{s}$. As the applied potential in the $\mathrm{CV}$ scans crosses the formal potential of the MB-labeled Ab molecule (at about - $0.12 \mathrm{~V}$ ); it triggers an associated optical absorption change that is clearly detected. The results shown in Figure 4-7 represent the optical absorbance data (red trace) collected when the EW-CRDS device functionalized with APTES and primary $\mathrm{Ab}$ was exposed to HA virus antigen with concentration $200 \mathrm{ng} / \mathrm{ml}$. As expected, the strong transition in the absorbance when the applied potential in the $\mathrm{CV}$ scans crosses the formal potential of the MB-labeled $\mathrm{Ab}$ molecule.

The black trace in Figure 4-7 corresponds to data collected when the virus antigen was absent from the solution. The negligible absorbance signal (and redox transition) in this data reports negligible amounts of MB-labeled Ab on the EW-CRDS surface when the virus protein is absent; this confirms that non-specific adsorption of the probe has been kept to a minimum at the functionalized interface. The experimental results in Figure 4-7 confirm the ability of the EW-CRDS platform to detect the presence and absence of the HA virus protein through spectroelectrochemical changes in the redox probe.

The measured absorbance in Figure 4-7 allows the determination of the total surface density of the adsorbed probe species in its oxidized state using Equation $\Gamma_{O X}=A_{O x} /\left(\mathrm{S} \epsilon_{O X}\right.$ ) ; such calculation gave us a value of $\Gamma_{O x}=(11 \pm 0.4) \mathrm{pmol} / \mathrm{cm}^{2}$. In addition, the fraction of species that converted to the reduced state can be determined using Equation 3-6; this 
calculation gave us a value of $\Gamma_{r e d}=(2.7 \pm 0.2) \mathrm{pmol} / \mathrm{cm}^{2}$. From these measurements one can determine the ratio of adsorbed species, $\Gamma_{\text {red }} / \Gamma_{o x}$, that undergoes redox transition during CV potential modulation; it was found that this ratio is $25 \%$. Previous calculations are based on values of molar absorptivity of oxidized and reduced forms of MB-labeled $\mathrm{Ab}$, and were measured by our group [88]; these values were found to be $\epsilon_{o x}=$ $4291 \mathrm{M}^{-1} \cdot \mathrm{cm}^{-1}$ and $\epsilon_{\text {red }}=205 \mathrm{M}^{-1} \cdot \mathrm{cm}^{-1}$.

The current density during the $\mathrm{CV}$ potential modulation can be calculated using the equation:

$$
I_{F}=\frac{n F v}{s \Delta \epsilon} \frac{d A}{d V}
$$

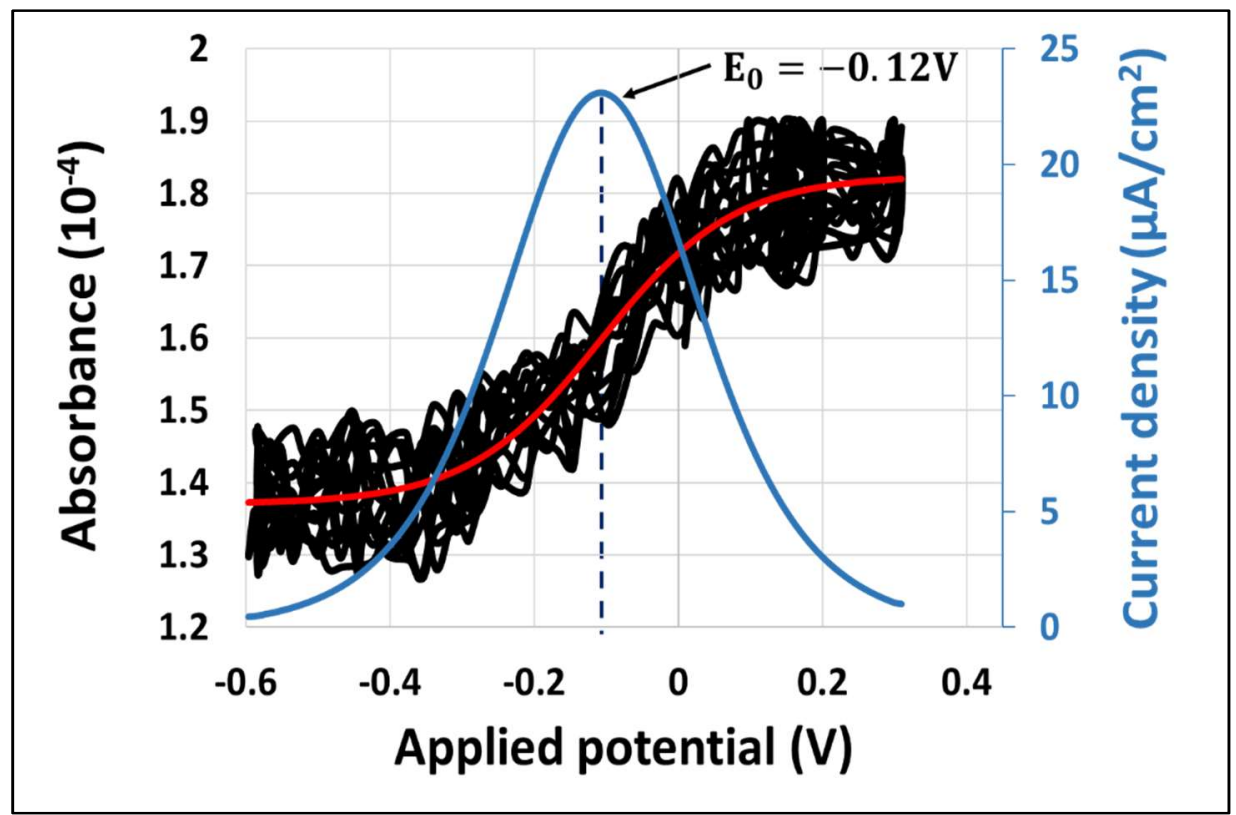

Figure 4-8: (Black trace) Modulated absorbance at $532 \mathrm{~nm}$ from the data presented in Figures 4-7 with a curve fitting (red trace) and the current density corresponding to the fitted line (blue curve) to determine the formal potential. 
where $S$ is a sensitivity factor $(\mathrm{S}=3.78), v$ is scan rate, $n$ is the number of electrons transferred in each redox event, $F$ is the faradaic constant, $\frac{d A}{d V}$ is the derivative of the modulated absorbance, and $\Delta \epsilon$ is the difference in the molar absorptivity between reduced and oxidized forms of MB-conjugated Ab.

To estimate the formal potential of the adsorbed MB-conjugated Ab. As shown in Figure 4-8; the data presented in Figure 4-7 for the modulated absorbance in the presence of virus antigen was fitted (red trace) and plotted with the corresponding current density (blue trace) as a function of the applied potential. The formal potential, which corresponds to the maximum value of current density, was found to be approximately $-120 \mathrm{mV}$.

\subsection{Conclusion}

We showed here the development of the highly sensitive and selective electro-active EW-CRDS platform, for the hemagglutinin (HA) protein from the H5N1 influenza A virus detection, in combination with a biologically specific sandwich immunoassay based on an electrically modulated optical signal generated by a redox probe. The advantage of the EW-CRDS method of measuring the optical absorption loss (and thus concentration) directly in real-time enable the investigation of binding kinetics of the electro-optical reporter that is highly selective through the specific interaction between capture $\mathrm{Ab}$ and a virus antigen. We demonstrated the ability of the conductive and transparent photonic device to detect the influenza virus antigen with a remarkable limit of detection of $280 \mathrm{pM}$. 
The electrically modulated optical signal collected with an electro-active EW-CRDS platform under CV potential modulation demonstrated the capability of our device for fast detection and direct quantification of the influenza antigen.

Moreover, the experimental results demonstrated the potential of the electro-active EW-CRDS device for high sensitivity detection of surface-confined targets at a single spot and even at very low concentrations of a target antigen. The detection capabilities demonstrated using the EW-CREDS offer a new path towards an arrayed-detection technology, which may be universally applied to detect a wide range of targets, ranging from DNA to proteins and small molecules. This type of immunosensor should be feasible in use for the field of molecular diagnosis and other life science fields in the future. 


\section{CHAPTER 5}

\section{HIGHLY SENSITIVE TRACE DETECTION OF NITRITE IONS IN WATER USING LIQUID-PHASE CAVITY RING-DOWN SPECTROSCOPY}

\subsection{Introduction}

Nitrite is commonly a pollutant ion. A trace amount of nitrite ions can be found in surface and ground water, due to the contamination of water sources by fertilizer, pesticides, and other organic waste. The concertation of nitrite is an important parameter in water quality [105-107]. Even a trace amount of nitrite ions can cause several serious health problems, such as decreased function of the thyroid gland, stomach cancer, and bluebaby syndrome in infants $[106,108-111]$. The maximum allowable amount of nitrite in drinking water is $100 \mathrm{ng} / \mathrm{ml}$ [107]. Therefore, methods for rapid and accurate detection of nitrite in water are required.

Traditional methods to detect nitrite ions in drinking water are based on the laboratory analysis [112]. However, these methods are time consuming and they use chemical reagents that cause secondary pollution. The most common methodologies to detect nitrite include ion chromatography [113], spectrophotometry [111, 114], kinetic methods [115, 116], flow-injection method [117], and electrochemical methods [118-120], etc.. Although most of these methods have high sensitivity, they are susceptible to interferences from the physical and chemical properties of detection samples, such as color, 
turbidity, and the interference from other compounds [121]. Furthermore, these methods are complicated and slow, and require sampling and sample pretreatment [122]. Recently, many spectroscopic techniques have been applied to detect nitrite in water such as infrared spectroscopy [122], Raman spectroscopy [106, 121] , and spectrofluorometry [123]. The infrared spectroscopy method suffers from limited sensitivity and is not suitable for the analysis of trace substances. The Raman signal is very weak; the effective cross section is very small, so low number of scattered photons are available for detection [106]. The low sensitivity of fluorescence spectroscopy makes the detection of concentrations in the lower to mid ppb range not possible [123].

Because of the fundamental role of nitrite ion in human health, still, there is need to new, simple, sensitive, powerful, and fast analytical tools for the determination of trace amount of nitrite ion in environmental samples such as natural waters (hot and cold spring water), wastewater and foodstuffs. The exploratory studies mentioned in Section 1.3.3.1 indicated that CRDS is promising for detection in samples and probing absorption in the liquid phase.

In this Chapter, we study a novel analytical method for the highly sensitive and selective liquid phase CRDS-based detection of nitrite ions in water. This method is based on the reducing a chromogenic indicator with nitrite ions in an acidic medium, which caused the purple dye color of the solution to change to yellow. The change in optical absorbance was measured by CRDS technique. With the normal incidence cuvette geometry, we report detection limits that are significantly lower than those achieved with a conventional absorbance methods. 
5.2. Principle of nitrite ions detection

Nitrite detection in water is based on the reduction reaction of Cresyl violet acetate (CV-Ac) dye, which is chemically known as 9-Amino-5-imino-5H-benzo[a]phenoxazine acetate, with nitrite ions in acidic medium. The CV-Ac was used as a chromogenic

(a)

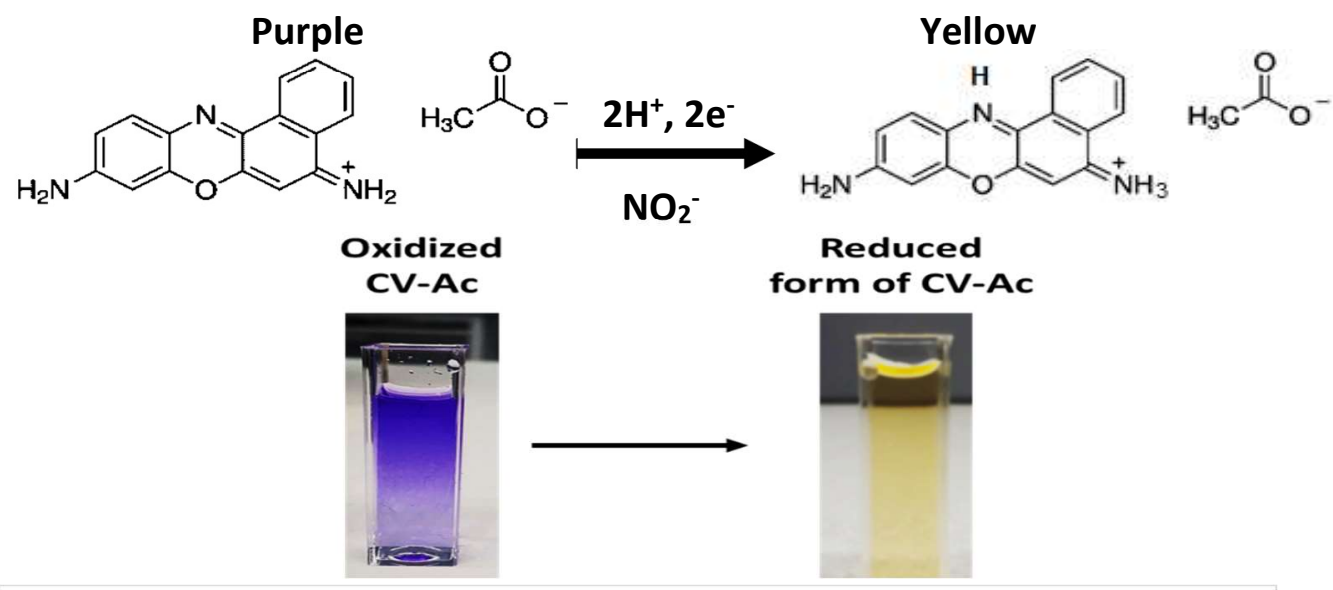

(b)

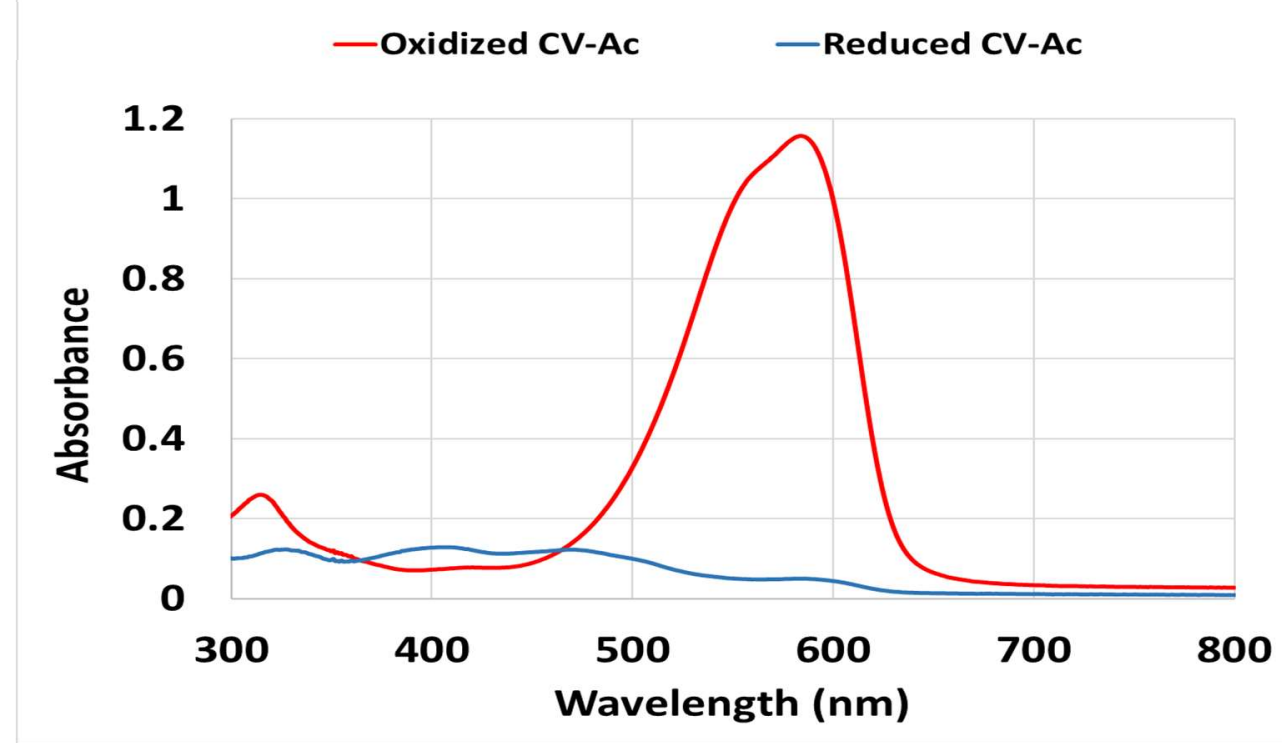

Figure 5-1: (a) The observed color change from purple to yellow due to the reduction of CV-Ac with nitrite ions in acidic medium. (b) UV-Visible spectra of CV-Ac and its reduced form in the wavelength range of $300-800 \mathrm{~nm}$. The optimum conditions: $5 \mu \mathrm{M}$ CV-Ac, $2 \mathrm{M} \mathrm{H}_{2} \mathrm{SO}_{4}, 1 \mu \mathrm{g} / \mathrm{ml} \mathrm{\textrm {NO } _ { 2 }}{ }^{-}$and $15 \mathrm{~min}$ at room temperature. 
indicator where its color turn to a yellow-colored reduced form as described in Figure 51(a); this will show a decrease in the optical absorbance with an increase of nitrite concentration at $532 \mathrm{~nm}$.

Figure 5-1(b) shows the UV Vis spectra of CV-Ac and its reduced form; the CV-Ac was reduced chemically by adding $1 \mu \mathrm{g} / \mathrm{ml}$ nitrite $\left(\mathrm{NO}_{2}^{-}\right)$to $5 \mu \mathrm{M}$ of CV-Ac in the presence $2 \mathrm{M}$ of sulfuric acid $\left(\mathrm{H}_{2} \mathrm{SO}_{4}\right)$. The method was successfully applied to the determination of nitrite ions in water samples.

\subsection{Experimental Section}

\subsubsection{Experimental setup}

As described in Figure 5-3, the experimental setup is the same as described in section 3.3.1. In this experiment, the quartz cuvette was mounted on ultra-precision alignment

\section{Aperture $1 \mathrm{~mm}$}

\section{Nd-YAG Pulsed Laser} $532 \mathrm{~nm}$

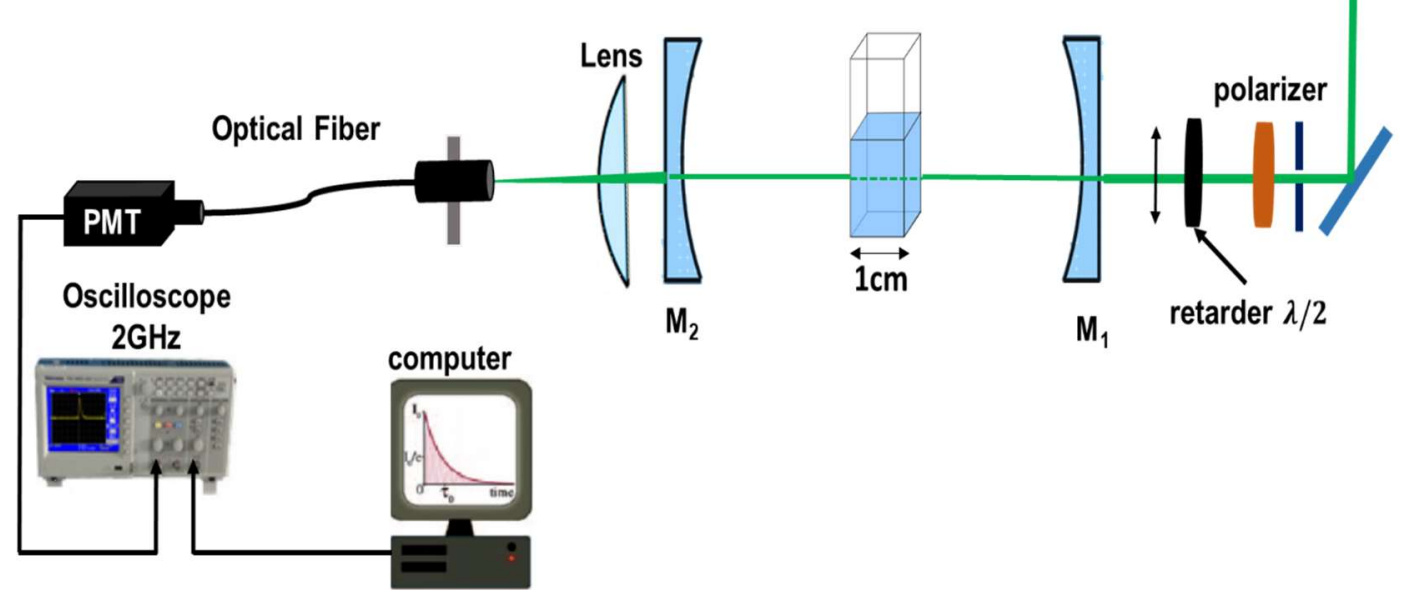

Figure 5-2: Schematic representation of the liquid phase CRDS setup. The quartz cuvette was inserted under normal incidence inside the optical cavity. 
stage (M-562F-XYZ-LH, Newport) and placed under normal incidence. As an advantage of this configuration, the reflection losses can be avoided by coupling the reflected light, on both sides of the cuvette, back to the cavity. For such configuration, when the cuvette was filled with DI water, the ring-down time water was $(3.6 \pm 0.04) \mu \mathrm{s}$. Moreover, as mentioned in Chapter 2, this geometry provided sensitivity to the absorption coefficient measurement as low as $1 \times 10^{-6} \mathrm{~cm}^{-1}$.

\subsubsection{Chemicals and Reagents}

All reagents and samples were diluted in DI water. A stock solution of CV-Ac (C5042, Sigma Aldrich) with concentration100 $\mu \mathrm{M}$ was prepared by dissolving $0.321 \mathrm{~g}$ of $\mathrm{CV}$-Ac in $10 \mathrm{ml}$ DI water. A Stock nitrite solution $(100 \mu \mathrm{g} / \mathrm{ml})$ was prepared by dissolving $1 \mathrm{mg}$ sodium nitrite in $10 \mathrm{ml} \mathrm{DI}$ water and diluting to different concentrations, $0.01-1,500$ ng/ml. Sulfuric acid (2 M) was prepared by introducing $55.5 \mathrm{~mL}$ concentrated $\mathrm{H}_{2} \mathrm{SO}_{4}$ (95$97 \%, \mathrm{~d}=1.84 \mathrm{~g} / \mathrm{ml}$ ) into a $500 \mathrm{~mL}$ volumetric flask and diluting to the mark with DI water. The reagent dye solution was stored in a brown bottle in a refrigerator.

\subsection{Results and Discussions}

5.4.1. The effect of sulfuric acid and CV-Ac dye concentration

To develop a quantitative analytical method for the detection of nitrite ions, preliminary studies were carried out to determine the most effective and optimum experimental conditions. The effect of $\mathrm{H}_{2} \mathrm{SO}_{4}$ acid concentration on the change of the absorbance was studied in the range 0.001-2.5 M sulfuric acid. The background signal of the ring-down time was recorded using a custom-made LabView program; the ring-down 
time was taken in the absence of acid and the presence of $1.5 \mathrm{ml} \mathrm{CV}-\mathrm{Ac}$ of concentration $5 \mu \mathrm{M}, 1 \mathrm{ml} 1 \mu \mathrm{g} / \mathrm{ml}$ nitrite and $1 \mathrm{ml}$ DI water, in the cuvette. The time average of this data was used to determine $\tau_{o}$. The analytical signal of ring-down time $(\tau)$ was recorded in the presence of $1 \mathrm{~m} \mathrm{H}_{2} \mathrm{SO}_{4}$ acid with concentrations in the range 0.001-2.5 $\mathrm{M}$, the same concentrations of the dye and nitrite were used. A waiting time of $15 \mathrm{~min}$ was used to complete the reaction, and this time was estimated by monitoring the reduction reaction in real-time using the CRDS device; Figure 5-4 shows that the time of $15 \mathrm{~min}$ is adequate to complete a chemical reaction. The ring-down times in presence $(\tau)$ and in the absence $\left(\tau_{o}\right)$ were used to determine the change in optical absorbance using Equation 3-1. Figure 5-5

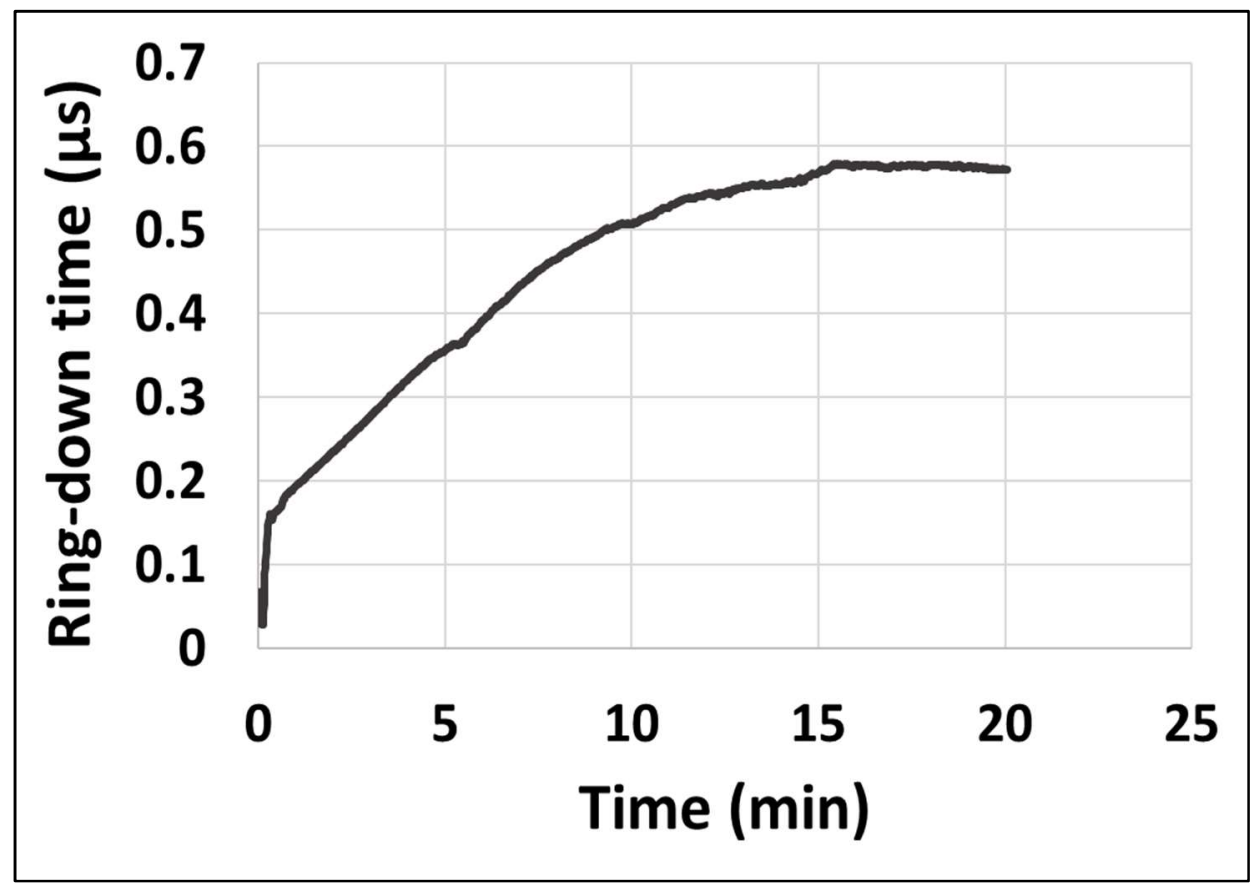

Figure 5-3: The reduction of CV-Ac dye with nitrite ions was optically monitored in real time by CRDS tool.

shows the influence of sulfuric acid concentration on the change on the absorbance $(\Delta \mathrm{A})$ during the reduction reaction of $\mathrm{CV}$-Ac with nitrite ion. The results show that the change 
of absorbance increased by increasing the sulfuric acid concentration until $1 \mathrm{M}$, then the reaction reaches the equilibrium state. At higher acid concentrations, $\mathrm{CV}$-Ac is protonated, thus reducing the rate of reduction reaction in the presence of nitrite ion [124]. The optimum concentration of $\mathrm{H}_{2} \mathrm{SO}_{4}$ was chosen as $1 \mathrm{M}$ in the final solution.

From the results obtained in Figure 5-5, we found that $5 \mu \mathrm{M}$ of $\mathrm{CV}$-Ac dye was sufficient to obtain a maximum reaction rate. Greater amounts of the $\mathrm{CV}$-AC cause a decrease in the reaction rate. This is due to the fact that in higher concentrations of CV-Ac in acidic media, CV-Ac coagulated and precipitated in the solution [124]. Thus, $5 \mu \mathrm{M}$ of CV-Ac was selected as an optimal concentration for analytical purposes.

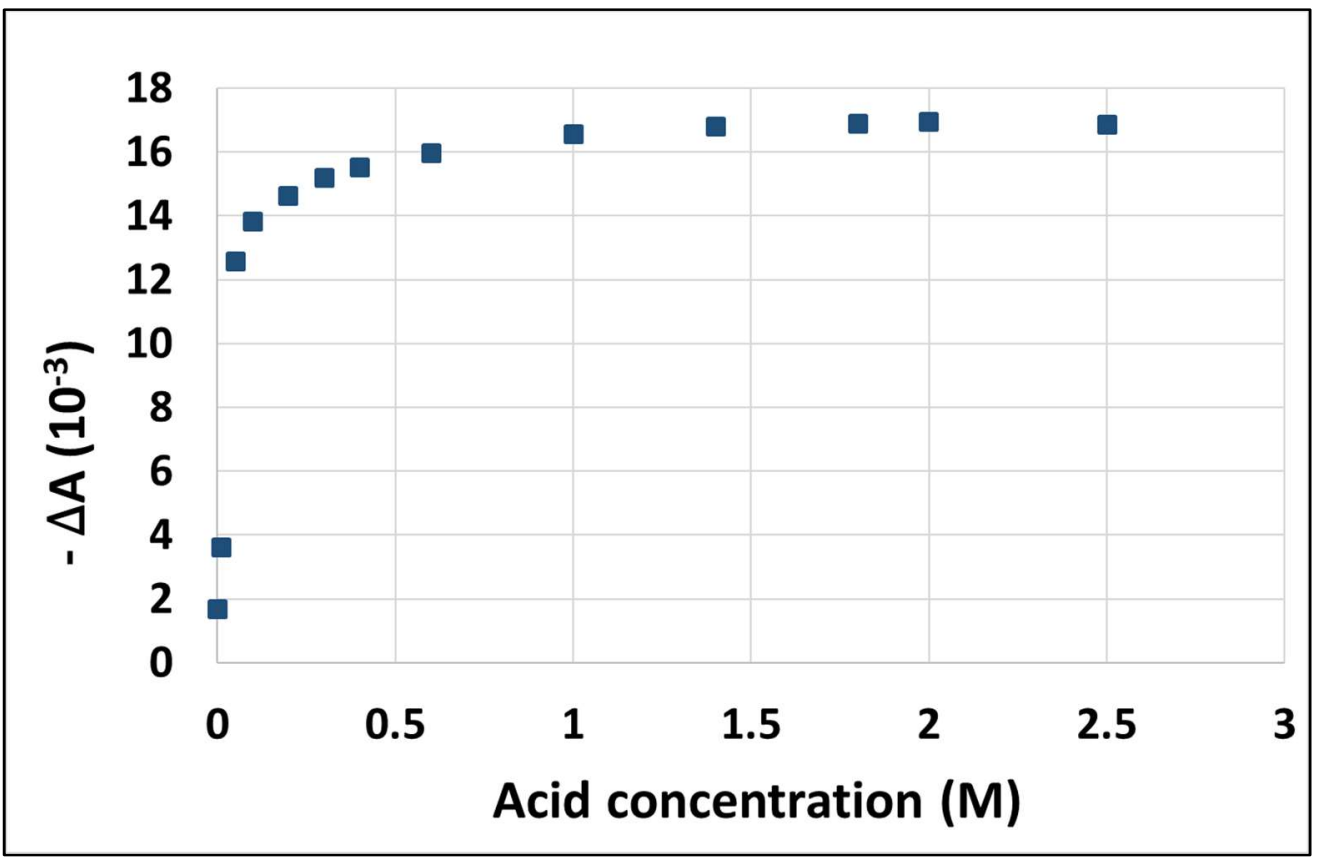

Figure 5-4: Effect of sulfuric acid concentration on the change of absorbance during the reduction of $\mathrm{CV}$-Ac dye with nitrite ions. The $\mathrm{CV}$-Ac concertation was $5 \mu \mathrm{M}$ and the nitrite ion concentration was $1 \mu \mathrm{g} / \mathrm{ml}$. 


\subsubsection{Nitrite ions detection in water}

An aliquot of the sample solution containing $0.05-1,500 \mathrm{ng} / \mathrm{ml}$ of nitrite was transferred to a series of $10 \mathrm{~mL}$ calibrated tubes. To this solution, $1.5 \mathrm{~mL}$ of $5 \mu \mathrm{M} \mathrm{CV}$-Ac and $1 \mathrm{~mL}$ of $1 \mathrm{M} \mathrm{H}_{2} \mathrm{SO}_{4}$ were added. A typical waiting time of 15 minutes was used to allow the reduction reaction to go to completion. Then, each sample was transferred to the quartz cuvette, and the ring-down time $(\tau)$ was immediately and continuously acquired for 15 minutes. The background ring-down time was recorded for the blank sample, where thesample was prepared in the same way, excluding the nitrite. The absorbance changes of the

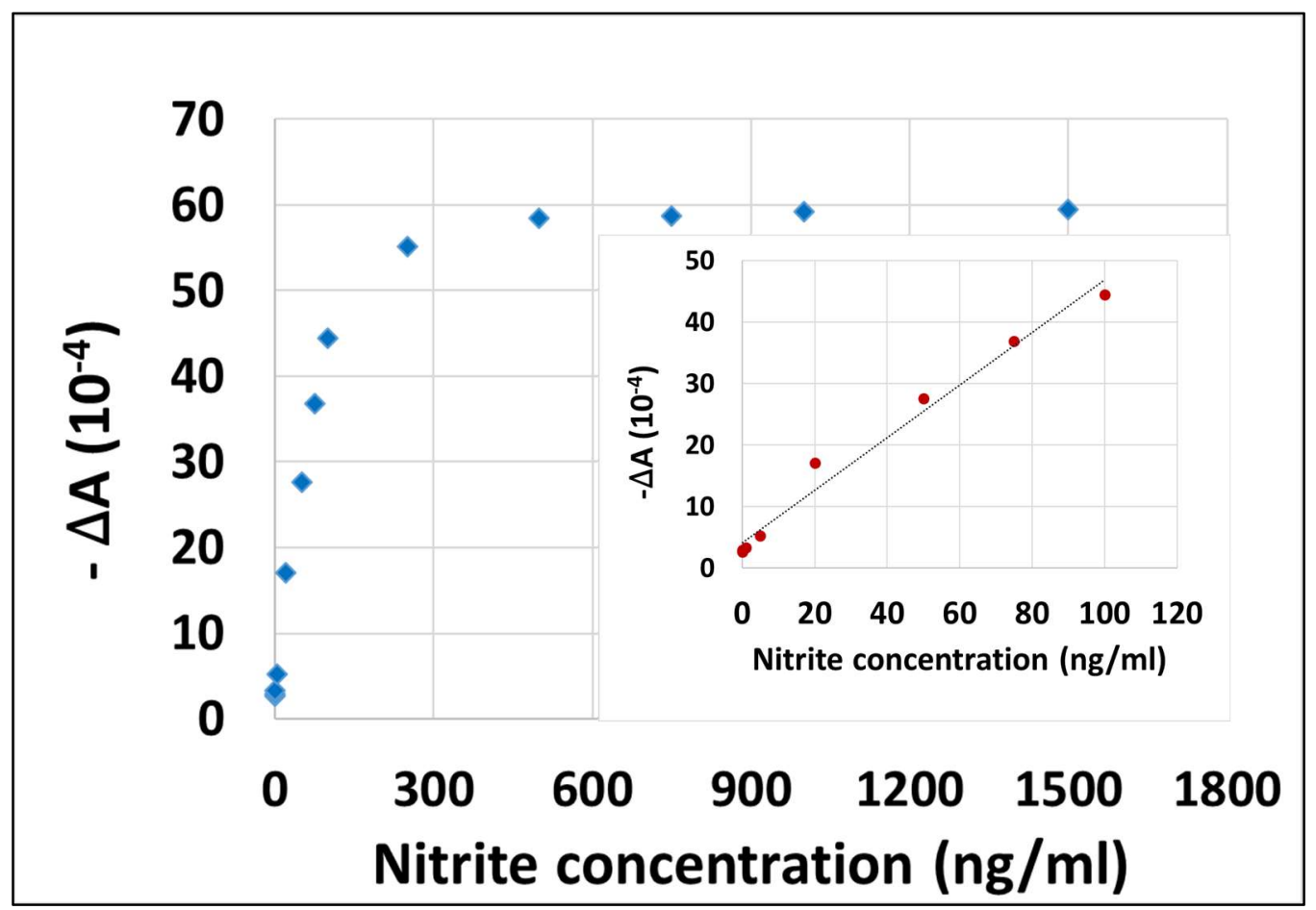

Figure 5-5: The change in the optical absorbance against various concentrations of nitrite ions at the wavelength $532 \mathrm{~nm}$. The inset is the calibration graph which displayed a strong linear relationship between the change in absorbance and the $\mathrm{NO}^{2-}$ concentration in the range from 0.05 to $100 \mathrm{ng} / \mathrm{ml}$. The optimal concentrations of CV-Ac dye and $\mathrm{H}_{2} \mathrm{SO}_{4}$ acid were 5 $\mu \mathrm{M}$ and $1 \mathrm{M}$, respectively. 
indicator dye and yellow colored-decay intermediate was obtained by subtracting the average ring-down time of the test solution $(\tau)$ from that of the blank $\left(\tau_{o}\right)$ using Equation 3-1. Figure 5-6 shows the absorbance change, $\Delta \mathrm{A}$, corresponding to the nitrite ion concentration; the calibration graph was linear in the range $0.05-100 \mathrm{ng} / \mathrm{ml}$ of nitrite as shown in the inset. The detection limit of $8 \mathrm{ng} / \mathrm{ml}$ or $120 \mathrm{nM}$ was calculated from the calibration graph, which is lower than what previously reported [105, 110, 121, 122, 125].

\subsection{Conclusions}

The highly sensitive liquid phase CRDS for trace detection of nitrite ions in water was demonstrated. An analytical method for the determination of trace nitrite $(0.05-1,500$ $\mathrm{ng} / \mathrm{ml}$ ) based on changing the color of CV-Ac dye from purple to yellow due to the reduction reaction of $\mathrm{CV}-\mathrm{Ac}$ with nitrite ions in acidic medium resulted in the decrease in absorbance. The decrease in absorbance is directly proportional to nitrite concentration. The reaction was monitored spectrally with CRDS by measuring the decreasing of absorbance at $532 \mathrm{~nm}$. The experimental results show that nitrite could be detected accurately within a low detection limit, which can reach as low as $8 \mathrm{ng} / \mathrm{ml}$. Moreover, with the current liquid phase CRDS setup, we achieved an absorption sensitivity as low as $1 \times$ $10^{-6} \mathrm{~cm}^{-1}$. In contrast to the spectrophotometry method and other spectroscopic techniques, this method demonstrated applicability in lower concentrations and higher selective detection. These advantages encourage the application of the proposed method in routine analysis in environmental water. 


\section{CHAPTER 6}

\section{CAVITY RING-DOWN SPECTROSCOPY STUDY ON THE UV SURFACE TREATMENT OF ULTRA-THIN INDIUM TIN OXIDE FILM}

\subsection{Introduction}

It has been demonstrated that CRDS is an effective technique for absolute small absorption losses measurement. As described in section 1.3.3.2, very few studies have been carried out to extend CRDS to the solid thin film. This could yield an extremely sensitive direct spectral absorption technique, which in principle can be applied in situ.

Obtaining a good optical transparency and the electrical conductivity simultaneously for ITO thin film is the crucial challenge for the successful implementation of various optoelectronic devices, such as solar cells [126-128], organic light-emitting diodes (OLEDs) [129, 130], liquid crystal displays (LCD) [131], flat panel displays (FPDs) [132], and spectroelectrochemical devices[47, 133, 134]. Several approaches have been used to fabricate high performance ITO film. For example, radio frequency (RF) or direct current (DC) magnetron sputtering $[135,136]$, chemical vapor deposition, pulsed laser deposition[137, 138], and atomic layer deposition (ALD) [139, 140]. 
However, the initial as-prepared ITO films do not have high transparency and low sheet resistance simultaneously.

Various methods have been developed to improve the electrical and optical properties of ITO films, such as conventional thermal annealing (CTA) treatments [141145], microwave annealing [146], chemical treatment [147], and plasma treatment [129, 148]. Besides those methods, UV treatment has been shown to enhance the performance of ITO film [149-151]. UV radiation decomposes molecular oxygen in the air to form ozone and atomic oxygen. Because ozone is a highly effective oxidizing agent it reacts quickly with organic contaminants and free radical molecules on the ITO surface; which changes the film constituents (In, Sn, O) ratio and increases the surface oxygen content, thereby enhancing the performance of the transparent electrode $[152,153]$. Most of the previous studies used UV treatment for relatively thick ITO films (above $100 \mathrm{~nm}$ ). However, this is not the case for the ultra-thin film (eg. below 30nm) [150-154] where the optical and spectroscopic properties cannot be precisely measured by conventional transmission and advanced techniques are needed in these cases. The reflectance and transmittance measurements of thin film can be very difficult when these quantities approaches unity [155].

In this chapter, we show the use of CRDS technique as a powerful tool for the measurements of the optical characteristics of ITO ultra-thin film under UV surface treatment. These properties are compared for samples as sputtered with and without UV radiation. The ITO films were treated with UV radiation after the films deposition and in situ during the films deposition processes in the sputtering chamber. CRDS technique was 
employed to measure precisely the minute change in optical absorbance of the film, while the electrical resistivity of the ITO films was measured simultaneously through the twopoint probe method.

\subsection{Experimental Section}

\subsubsection{ITO ultra-thin film samples preparation}

Before the film deposition process, the glass substrates were cleaned using the cleaning protocol mentioned in Chapter 3. Ultra-thin ITO film was deposited on top of glass slides using DC magnetron sputtering technique. A 3-inch target of $\left(\mathrm{In}_{2} \mathrm{O}_{3}\right) 90:(\mathrm{SnO}) 10 \mathrm{wt} \%$ and $99.99 \%$ purity was used as a sputtering target. Before deposition, the chamber was pumped down until the pressure reached about $5 \times 10^{-6} \mathrm{~Pa}$. The glass substrates were placed in the sputtering chamber for a sufficiently long time (15 minutes) to reach the deposition temperature $250{ }^{\circ} \mathrm{C}$. The rotation speed was $20 \mathrm{rpm}$ to create uniform films and a DC power of $230 \mathrm{~W}$ was applied with a frequency of $20 \mathrm{kHz}$ and a pulse duration of $1 \mu$ s. Subsequently, the working gases were introduced into the deposition chamber, the high purity argon was fixed at $100 \mathrm{sccm}$ and different flow rates of oxygen (from $0.6 \mathrm{sccm}$ to $4 \mathrm{sccm}$ ) were used. The deposition time of the $20 \mathrm{~s}$ was used to grow a film. Two types of samples were prepared (one with and the other without UV exposure) under the same conditions. The UVC LED Lamp with maximum emission at $275 \mathrm{~nm}$ and power $3 \mathrm{~mW}$ was used as a UV radiation source. To protect the lamp from coating, the lamp was placed inside the aluminum tube with length 2 inches and angle $45^{\circ}$ with a vertical plane. 


\subsubsection{CRDS Experimental Setup}

The experimental setup is the same as described in Section 3.3. As shown in Figure 6-1, the difference here, the glass substrate coated with ITO was mounted on 3-D translation with rotational stage (XR25P-K2, Thorlabs). The laser beam enters the cavity and hits the surface of the substrate at Brewster's angle of glass. For UV treatment, the UV LED lamp was mounted at a distance of $1 \mathrm{~cm}$ from the ITO sample.

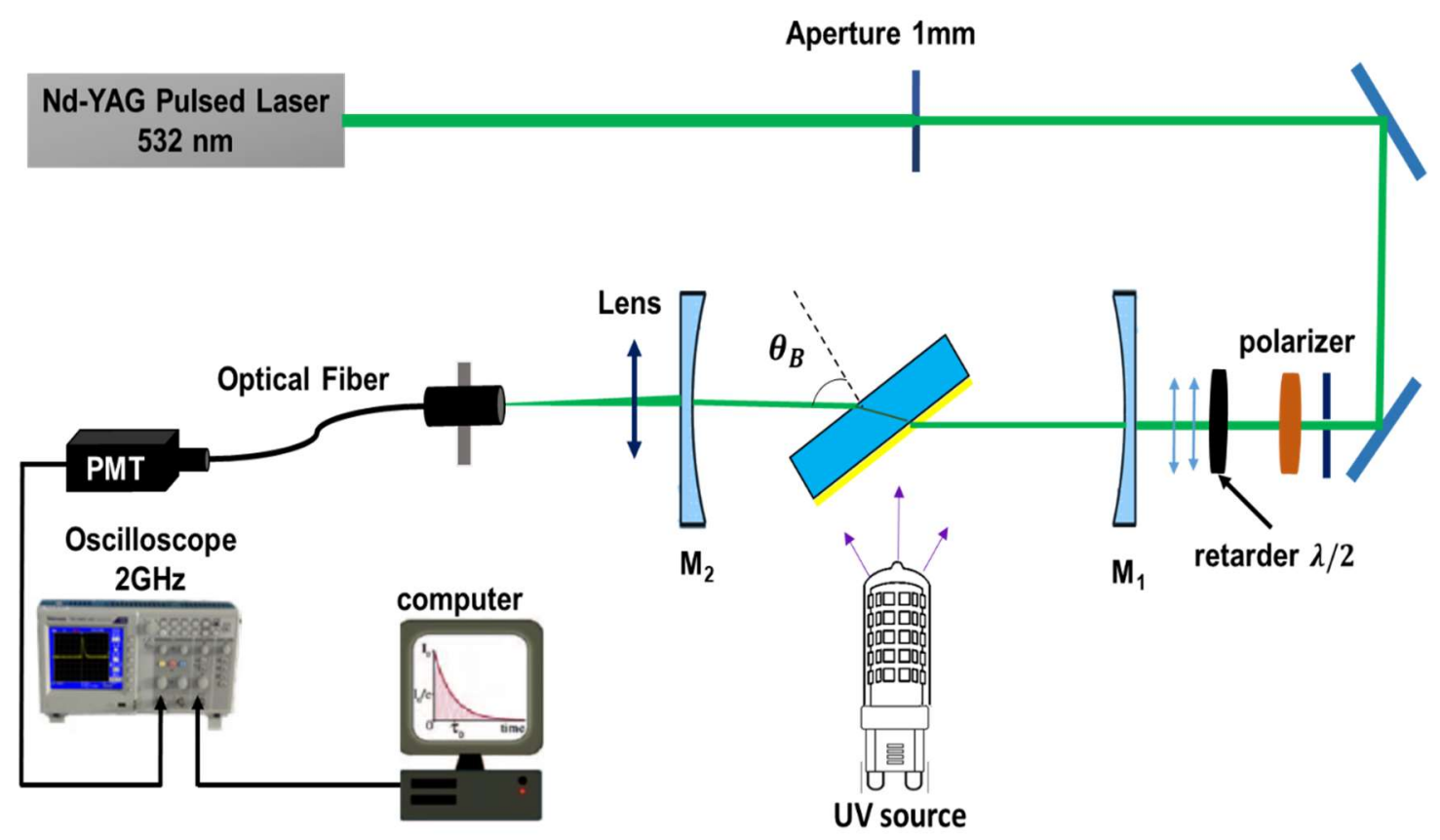

Figure 6-1: Scheme of the thin film CRDS experimental setup.

\subsubsection{Absorption and Transmittance Measurements of the Thin Film}

In the optical resonator shown in Figure 6-1, the round trip optical loss factor $e^{-\alpha_{r}(2 L)}$ can be given by [78]:

$R_{M_{1}} T_{1} R_{M_{2}} T_{2}=e^{-\alpha_{r}(2 L)}$ 
Where $R_{M_{1}}, R_{M_{2}}$ are mirrors reflectance, $T_{1}, T_{2}$ are a single pass transmittance through a thin film and glass substrate, $L$ is the cavity length, and $\alpha_{r}$ is an effective overall distributed-loss coefficient per unit length. By assuming $T=T_{1}=T_{2}$ and $R=R_{M_{1}}=$ $R_{M_{2}}$, then:

$R^{2} T^{2}=e^{-2 \alpha_{r} L}$

The ring-down time can be written as:

$\tau=\frac{1}{c \alpha_{r}}$

where $c$ is the speed of light. The change of absorbance introduced by the film during the UV exposure can be written as:

$\Delta A=\frac{L}{\ln (10) c}\left[\frac{\Delta \tau}{\tau \tau_{0}}\right]$

where $\tau_{0}$ and $\tau$ are the ring-down time without and with UV exposure respectively and $\Delta \tau=\tau_{0}-\tau$ is the change in the ring-down time.

The sheet resistance of the ITO film was measured in real time during UV exposure. This was performed by contacting the film with multi-meter as a two-point probe using adhesive copper tape to make proper electrical contact and reducing the contact resistance. For any three-dimensional conductor, the resistance can be used to obtain the resistivity according to the equation:

$$
R=\rho \frac{l}{\mathrm{tw}}=R_{s} \frac{l}{w}
$$


where $\rho$ is the resistivity, $l$ and $\mathrm{w}$ are the sheet length and width, $\mathrm{t}$ is the thickness, and $R_{S}$ is the sheet resistance (where $R_{s}=\rho / t$ ). As the film thickness is measured, the resistivity $\rho$ (in $\Omega . \mathrm{cm}$ ) can be calculated by multiplying the sheet resistance by the film thickness in $\mathrm{cm}$ :

$\rho=R_{s} \times \mathrm{t}$

\subsubsection{Optical Properties Measurements}

For getting the thickness and refractive index of each ITO samples were deposited with and without UV exposure. The transmittance and reflectance versus incident angle for both $\mathrm{s}$ and p-polarized light were measured for each sample. From the schematic representation in Figure 6-2, the experimental setup consists of a CW laser diode (Shanghai Dream lasers Tech., SDL-532-150T) at the wavelength of $532 \mathrm{~nm}$ and operated at $15 \mathrm{~mW}$. The laser beam was passed through a half-wave plate and linear polarizer in order to select the light polarization. It was then directed towards the ITO sample that was mounted on a rotation stage (Quadra-Chek 100) to precisely control the incident angle. The reflected and

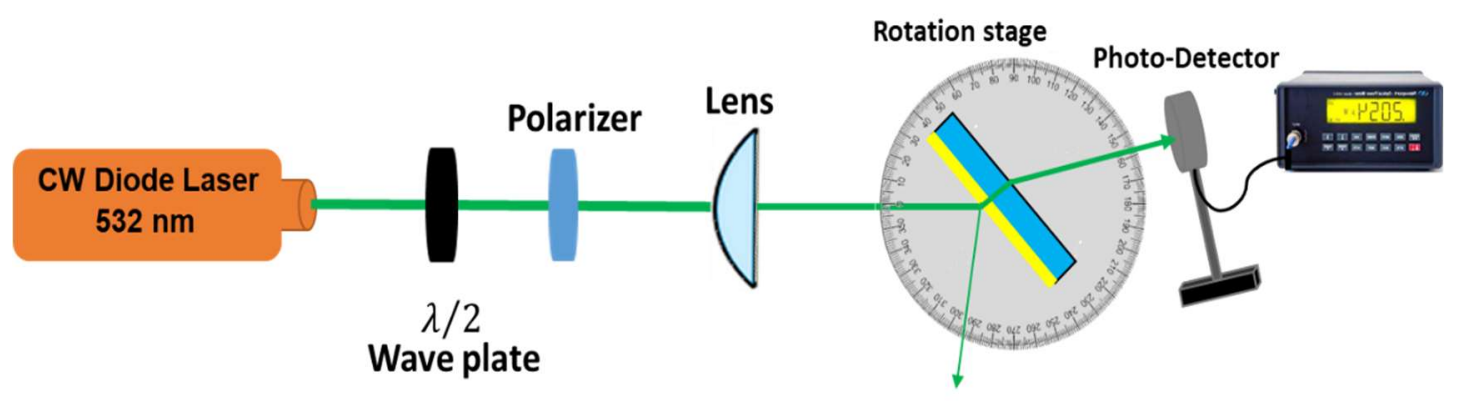

Figure 6-2: Schematic representation of the experimental setup used to measure the transmittance and reflectance versus the incident angle, for s and p-polarized light, of ITO ultra-thin film. 
transmitted optical intensity at different angles of incidence was monitored by a power meter (Newport model 1930C).
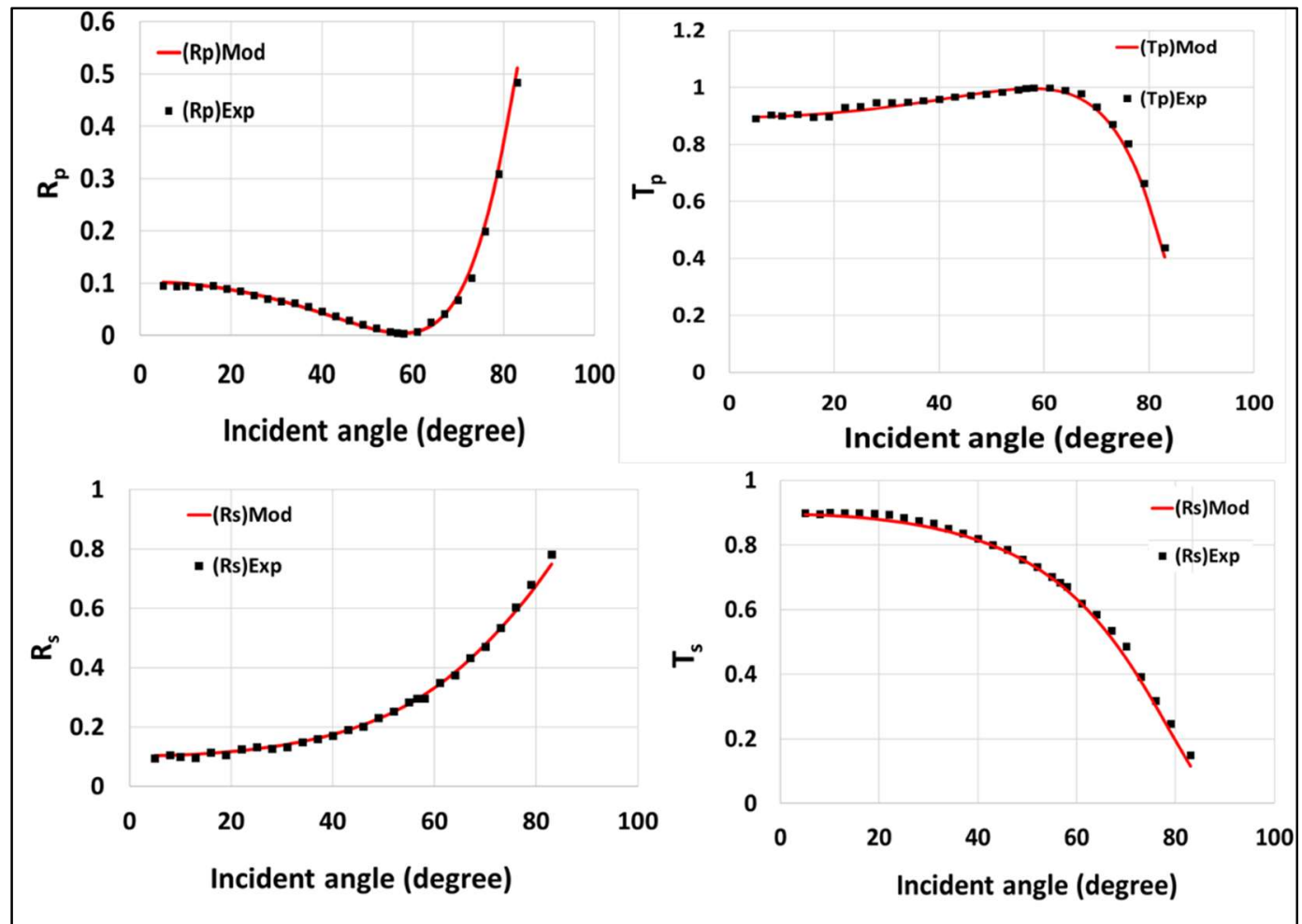

Figure 6-3: Reflectance and transmittance against incident angle for $\mathrm{p}$ and $\mathrm{s}$ polarized

light. The black dots are experimental results, and the red solid line is the modulation data.

To extract the thickness (t), real (n), and imaginary (extinction coefficient, k) parts of the refractive index, a Mathematica program using a transfer-matrix technique of multilayer structure was used by matching the modulated transmittance and reflectance with the experimental measurements (Mathematica program code is described in appendix 8(a)). The reflectance and transmittance spectra, for $\mathrm{p}$ and s polarized light, were plotted and compared to a model generated using known optical constants in Mathematica; an example is shown in Figure 6-3. In order to find the best parameters ( $\mathrm{t}, \mathrm{n}$, and $\mathrm{k}$ ), the values thickness and refractive index of the model ITO film can be manipulated to find a model 
transmittance and reflectance spectra that most closely matches the experimental measurements.

The program worked well to extract the thickness and the real part of refractive index, but it failed to convert the extinction coefficient; the reason being that the values of $\mathrm{k}$ are very small for the ultra-thin film. To overcome this hurdle, the ring-down time was measured for each sample coated with and without UV exposure and for each $\mathrm{O}_{2}$ flow rate, then the measured ring-down times were converted to single-pass transmittance for $\mathrm{p}$ polarized light at $532 \mathrm{~nm}$ using Equations 6-2 and 6-3 (These results are summarized in appendix 9). The values of the thickness and the real component of refractive index of each sample that was extracted in the previous step were fixed, and the same Mathematica program with one data point (transmittance at p-polarization) was used to extract extinction coefficient of the films (the Mathematica program is described in appendix 8(b)).

\subsection{Results and Discussions}

\subsubsection{UV Treatment of ITO Ultra-Thin Film after the Sputtering Deposition Process}

Ultra-thin ITO film samples were prepared onto glass substrates using the same parameters as mentioned before, and $0.6 \mathrm{sccm}$ oxygen flow rate was selected. For the optical performance measurements, the ring-down time with and without UV exposure treatment was monitored in real time for $500 \mathrm{~s}$ by the CRDS platform. The effect of UV treatment can be clearly seen in Figure 6-4 (a); in the first $100 \mathrm{~s}$, the ring-down time increases rapidly with UV exposure and then reaches a steady state at a longer time; which indicates that there is no further improvements on the film surface. 


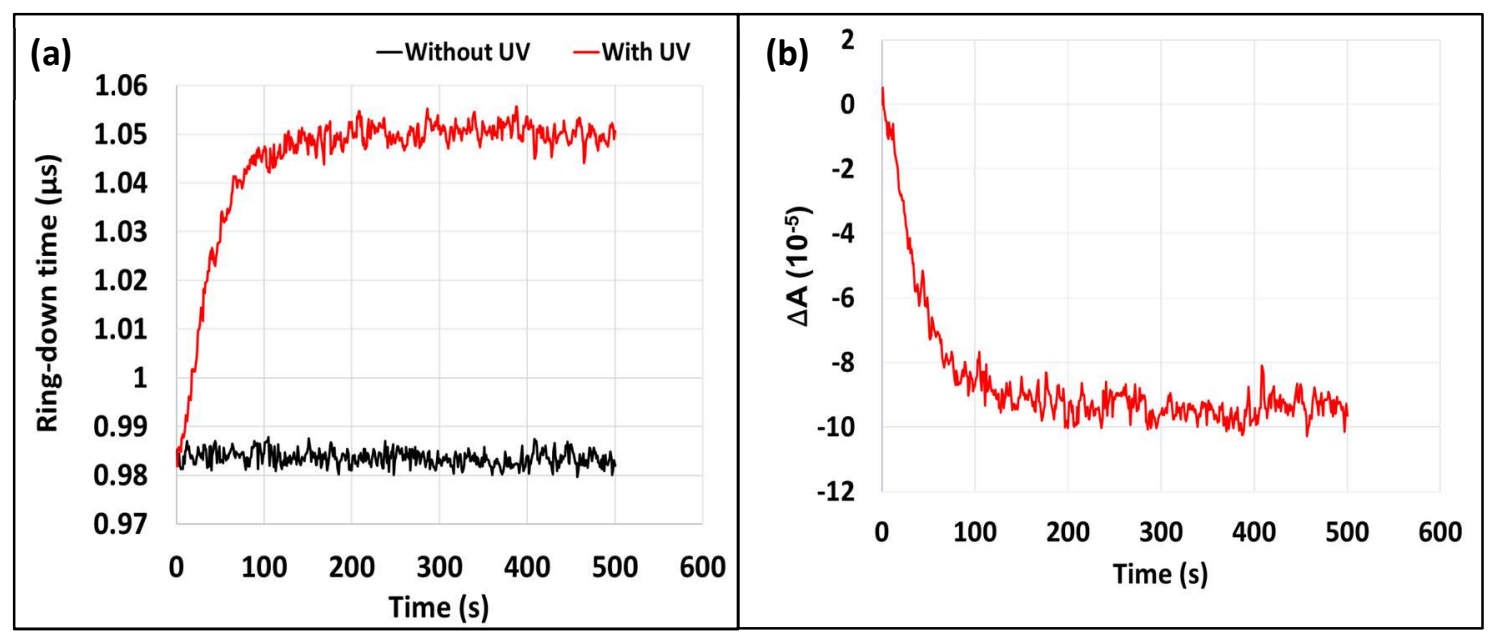

Figure 6-4: (a) Ring-down time data as measured by CRDS tool for ITO ultra-thin film with and without UV treatment, (b) Absorbance per pass in the cavity as a function of total time during UV treatment.

This can be interpreted to say that the oxygen vacancies are the main scattering loss source of the light in the ITO films. UV treatment works to oxidize the sub-oxides such as $\mathrm{InO}_{\mathrm{x}}$ and $\mathrm{SnO}_{\mathrm{x}}$ by adding $\mathrm{O}^{-2}$ ions into the ITO surface, which leads to decreased oxygen vacancies, and increased film optical transmittance $[156,157]$. The values of the ring-down time measured without and with UV exposure was converted to the change in absorbance per pass using Equation 6-4, as shown in Figure 6-4(b). The negative values of absorbance mean that UV exposure worked to increase the transparency of the ITO film. The optical Table 6-1: Real, imaginary parts of refractive index, and thickness of ITO ultra-thin film samples without and with UV treatment.

\begin{tabular}{|c|c|c|c|}
\hline & $\mathbf{n}$ & $\mathbf{k}$ & $\mathbf{t}(\mathbf{n m})$ \\
\hline Without UV & 1.9 & 0.0053 & 12.6 \\
\hline With UV & 1.9 & 0.0040 & 12.8 \\
\hline
\end{tabular}


measurements using the CRDS revealed that transmittance of the ITO film increased by $20 \%$.

Based on the method proposed in section 6.2.4, the values of thickness, real part of refractive index, and extinction coefficient are listed in Table 6-1. The sheet resistance was recorded in real time during UV exposure and was used to obtain the resistivity using Equation 6-6. As we observe in Figure 6-5, the UV treatment improves the film conductivity, where the resistivity drops from $4.8 \times 10^{-3} \Omega . \mathrm{cm}$ to $2.2 \times 10^{-3} \Omega . \mathrm{cm}$.

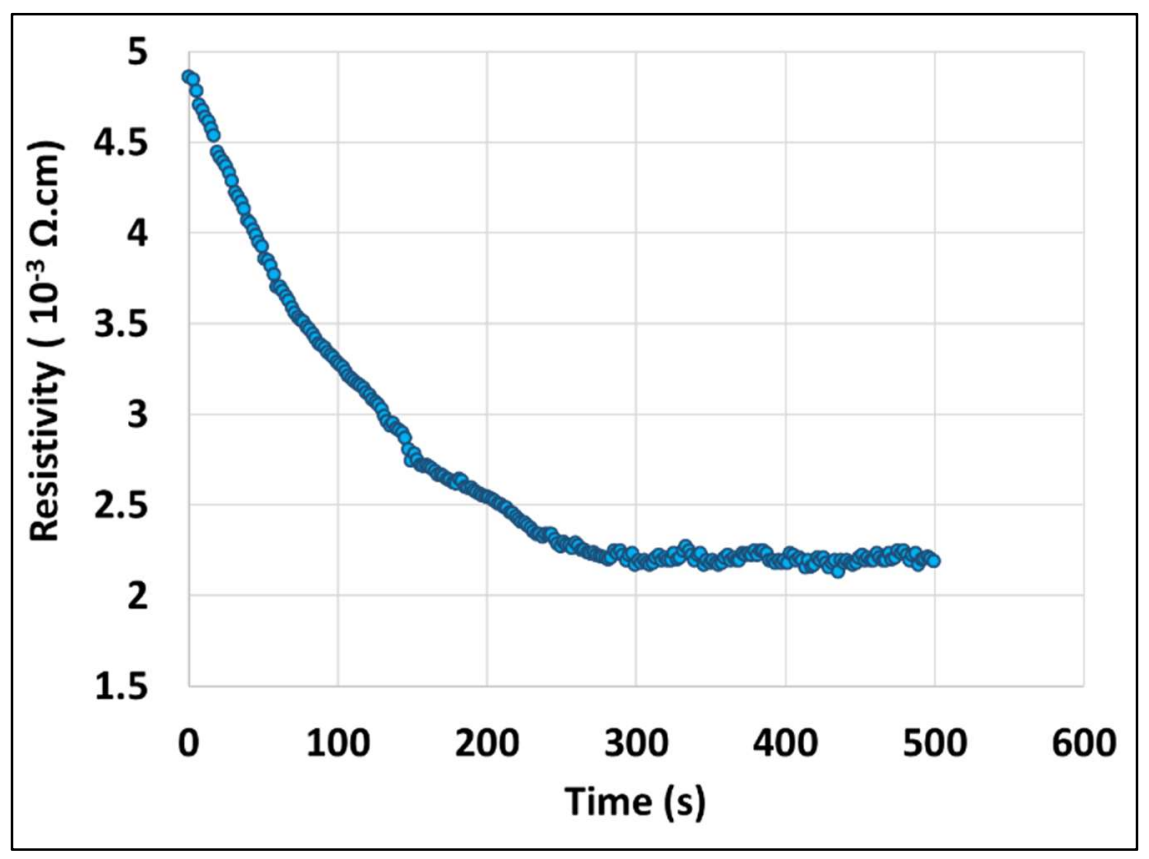

Figure 6-5: The variation of the electrical resistivity of ITO ultra-thin film in real time during UV treatment.

6.3.2. In situ UV Treatment of ITO Ultra-thin Film during the Sputtering Deposition Process

Figure 6-6 (a) and (b) shows the measured ring-down time and the extinction coefficient for the samples as deposited with and without $\mathrm{UV}$ radiation and under different $\mathrm{O}_{2}$ flow 


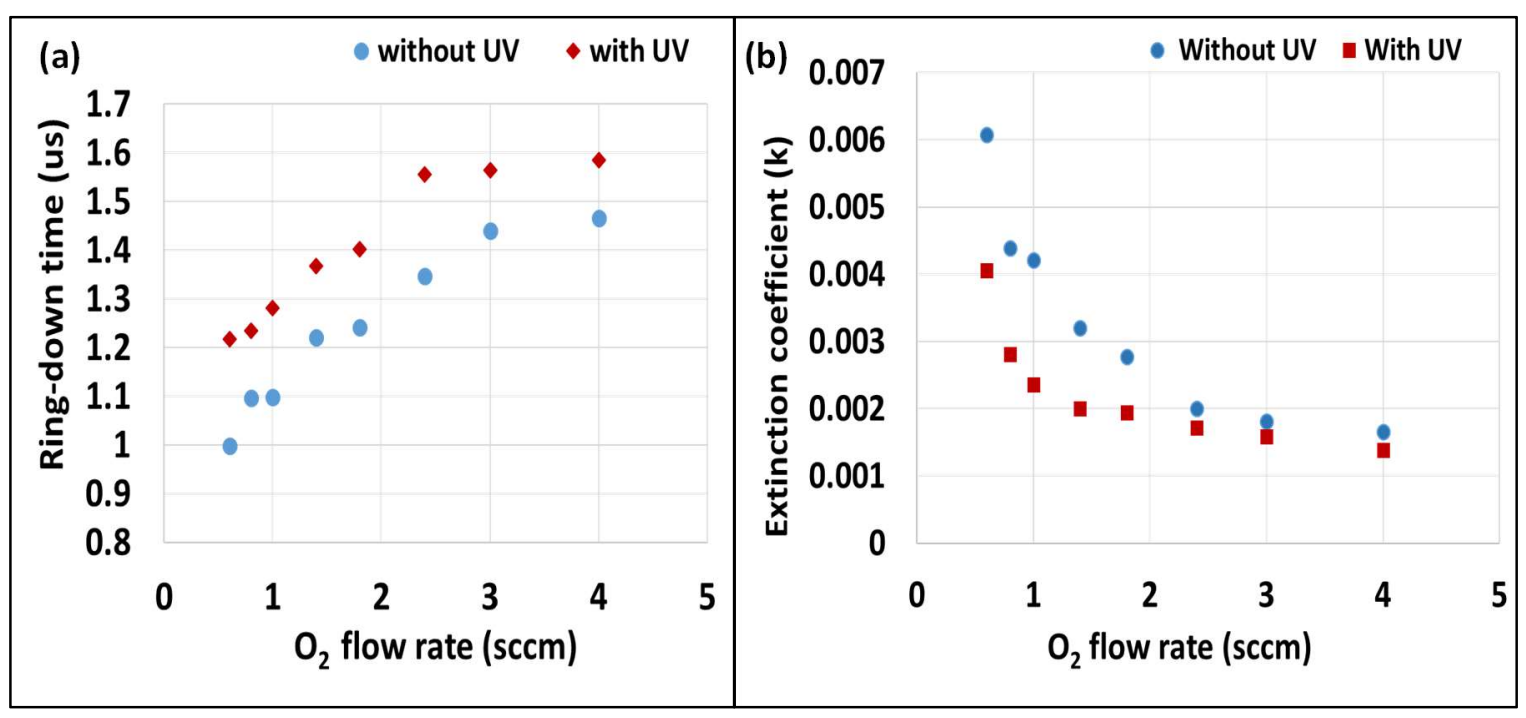

Figure 6-6: The change in (a) ring-down time, (b) extinction coefficient for ITO films as deposited with and without UV exposure at different oxygen flow rates.

(from 0.6 to $4 \mathrm{sccm}$ ). In general, it can be seen that the transmittance of ITO film increases (i.e. ring-down increases) and the extinction coefficient decreases with oxygen flow rate. At low $\mathrm{O}_{2}$ flow rate the oxidation of the ITO film surface is incomplete and the prepared film become anoxic, so the number of the oxygen vacancies goes up and this leads to a low transmission (i.e. a high extinction coefficient). On the other hand, the high $\mathrm{O}_{2}$ flow rates decrease the oxygen vacancies which lead to a high transmittance (i.e. a low extinction coefficient) of the ITO film $[157,158]$. As we observe in Figure 6-6 (a), the UV exposure during the deposition process further increases the film transmittance (i.e. decreases the extinction coefficients). By comparing the change in transmittance and electric resistivity obtained in section 6.3 .1 (at $0.6 \mathrm{sccm}$ ) with those values for the samples coated in the presence of UV radiation in the sputtering chamber, we conclude that the UV treatment during the film deposition is more effective for improving the properties of the ITO thin film. 
The electrical resistivity of these ITO films has been plotted in Figure 6-7. In general, it can be seen that ITO films showed a monotonic increase in their resistivity as the $\mathrm{O}_{2}$ flow rate is increased, whereas the films prepared under $\mathrm{O}_{2}$ flow rate from 0.6 to $1.4 \mathrm{sccm}$ have low electrical resistivity values. The results show that the resistivity becomes very large $\left(1.2 \times 10^{-1} \Omega . \mathrm{cm}\right)$ at $4 \mathrm{sccm}$, so it has been excluded in Figure 6-7. This is can be

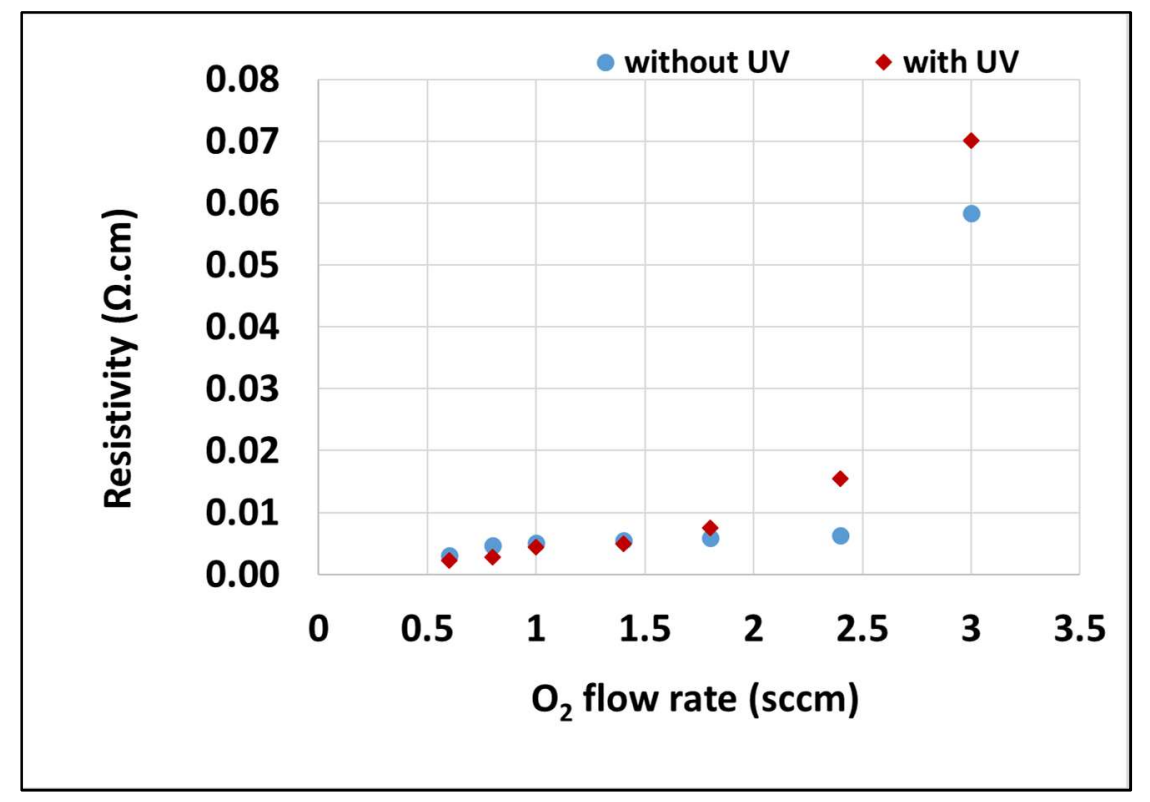

Figure 6-7: Electrical resistivity of ITO ultra-thin film samples as deposited with and without UV exposure under different oxygen flow rates.

explained by the fact that as the $\mathrm{O}_{2}$ flow is increased, the number of the oxygen vacancies is decreased, which result in the decreasing of the charge carriers concentration and leads to a high resistivity $[151,156,159]$. Furthermore, we notice discrete changes of the electrical resistivity of ITO films prepared with UV exposure; at low $\mathrm{O}_{2}$ flow rate in the range of $0.6-1.4 \mathrm{sccm}$, the UV environment slightly decreases the electrical resistivity with the minimum value of $2.4 \times 10^{-3} \Omega . \mathrm{cm}$ at $0.6 \mathrm{sccm}$. Although the transmittance of the deposited samples increases significantly at the $\mathrm{O}_{2}$ flow rate over $1.4 \mathrm{sccm}$, the resistivity 
shows a monotonic increase in their values; this can be attributed to a highly oxidant $\mathrm{O}_{3}$ atmosphere that possibly decreases the concentration of oxygen vacancies (decrease the carrier concentrations) in ITO, thereby contributing to raising the electrical resistivity [159].

By considering both the transmittance and the electrical resistivity, we observe that the $\mathrm{O}_{2}$ flow rate in the range of $0.6-1.4 \mathrm{sccm}$ is the optimum value for getting ultra-thin films with good transmittance and low electrical resistivity. The results showed that the resistivity becomes very large $\left(1.2 \times 10^{-1} \Omega . \mathrm{cm}\right)$ at $4 \mathrm{sccm}$, so it has been excluded in Figure 6-7.

\subsection{Conclusions}

The potential of the CRDS technique to measure minute absorption loss, with a precision of better than $1 \mathrm{ppm}$, in the ITO ultra-thin film during UV treatment was demonstrated. With Brewster's angle substrate geometry, we have been able to measure optical loss sensitivity as small as $3 \times 10^{-6} \mathrm{~cm}^{-1}$. The ITO films were treated with UV radiation after and during the deposition process at different oxygen flow rates. The measurements of optical constants and cavity ring-down times show that the UV treatment increase the transparency of ITO ultra-thin film by about $20 \%$. According to the results, the in situ UV treatment in the sputtering chamber decreases the film resistivity in the $\mathrm{O}_{2}$ flow rate in the range of $0.6-1.4 \mathrm{sccm}$. However, it increases unevenly for the oxygen flow rate over $1.4 \mathrm{sccm}$. This prompts us to conclude that the optimum values of oxygen flow rates for getting the films with good transmittance and low electrical resistivity are in the range of 0.6-1.4 sccm. 


\section{CHAPTER 7}

\section{SUMMARY AND OUTLOOK}

\subsection{Summary}

This dissertation aimed to develop and apply novel extremely sensitive modes of absorption spectroscopy, condensed phase CRDS platforms in order to study a wide range of interfacial phenomena. To accomplish this, several variations of these instruments were built and modified to minimize the optical losses throughout the resonant cavity. The performance of each condensed phase CRDS configuration was determined by calculating the minimum detectable absorption coefficient.

The development of an electrically-active solid/liquid interface for the EW-CRDS technique to enable spectroelectrochemical investigations of redox events was described. A high sensitive electro-active EW-CRDS platform was achieved by creating a highquality, ultra-thin, transparent conductive (to minimize optical loss and to support potential modulations) electrode film of ITO on the interface of total internal reflection, which enabled us to perform spectroelectrochemical studies at solid/liquid interfaces. As a proofof-concept on the capabilities of the developed platform, measurements were performed to address the effects of an applied electric potential to the adsorption behavior and affinity strength of a redox species onto different interfaces. The results for a synchronized EWCRDS measurements with the CV scan demonstrated the ability of the EW-CRDS platform 
to investigate the electrode-driven redox events of a molecular species at low submonolayer coverages. The total surface coverage and active surface coverage were calculated based on the spectroscopic measurement during the $\mathrm{CV}$ potential scan. The ability of electro-active EW-CRDS to monitor the interaction of biomolecules with different molecular assemblies of a modified electrode in situ and in real time, expands the applicability of EW-CRDS to develop a novel bio-sensing technology based on the combination of the EW-CRDS with a biological immunoassay displaying redox activity. The advantage of the EW-CRDS method of measuring the optical absorption loss (and thus concentration) directly in real-time, enable the investigation of binding kinetics of an electro-optical reporter that is highly selective through the specific interaction between capture $\mathrm{Ab}$ and a virus antigen. We demonstrated the ability of the conductive and transparent photonic device to detect the $\mathrm{H} 5 \mathrm{~N} 1$ influenza A virus antigen with a remarkable limit of detection of $280 \mathrm{pM}$. Moreover, the experimental results demonstrate the potential of the electro-active EW-CRDS device for high sensitivity detection of surface-confined targets at a single spot and even at very low concentrations of a target antigen. Such performance using the EW-CREDS and the demonstrated detection capabilities has the potential to create a new bio-sensing technology capable of offering substantially shorter detection times with simpler and more cost-effective protocols, which are critical for pointof-care applications in disease diagnostics.

Finally, the development of an ultra-sensitive direct absorption technique based on CRDS to study the UV surface treatment effects on the optical properties of ITO ultra-thin film was investigated. The results demonstrated the ability of the CRDS to measure minute absorption loss in the ITO ultra-thin film during UV surface treatment; with Brewster's 
angle substrate geometry, we have been able to measure optical loss sensitivity as small as $3 \times 10^{-6} \mathrm{~cm}^{-1}$. The ITO film was treated with UV radiation after and during the deposition process at different oxygen flow rate in the range of $0.6-4 \mathrm{sccm}$. The measurements of optical constants and cavity ring-down times have shown that the UV treatment enhanced the transparency of ITO films.

\subsection{Outlook}

The applications reported in this work are expected to open several opportunities to investigate a variety of electrochemical phenomena at solid/liquid interfaces for the development of novel biomaterials, biosensors, and bioanalytical systems. The implementations of many applications using CRDS is limited by the excitation source specification such as laser wavelength and repetition rate, for Example, the pulsed laser sources operating at low repetition rate (e.g., $10 \mathrm{~Hz}$ ), which presents a significant limitations in performing some applications [160-162]. For example, the investigation of the electron transfer kinetics of surface immobilized redox species using optical impedance spectroscopy (OIS) measurements required laser source with high repetition rate [47, 163].

As a future work, it would be high interest to use continuous wave CRDS (CWCRDS) laser instead of pulsed laser sources. The spectrum of CW-CRDS provided several advantages over pulsed CRDS, including high repetition rate (in order of $\mathrm{kHz}$ ) which leads to more averaging and improved sensitivity [164], high spectral resolution and high SNR. $\mathrm{CW}$ lasers have a narrower bandwidth than pulsed lasers and analogous to single-mode CRDS. The cavity length should therefore be such that the laser frequency coincides with 
one of the cavity modes in order to achieve excitation [160-162, 164]. In CW-CRDS, a $\mathrm{CW}$ laser is used to excite the cavity and the ring-down signal is measured after switching of the laser, for example using a fast optical switch such as an acousto-optic modulator $(\mathrm{AOM})$ or electrical optical modulator $(\mathrm{EOM})$. It will be interesting to combine the electroactive CW EW-CRDS with the OIS, which might open new prospects for many electrochemical biosensing applications.

Another possible work is phase-shift cavity ring-down spectroscopy (PS-CRDS). As alternative of pulsed laser CRDS, PS-CRDS can be applied to improve electrochemical measurements. In PS-CRDS the optical cavity is excited with amplitude-modulated CW laser. To optimize the sensitivity, a fixed frequency is usually chosen close to $1 / 2 \pi \tau$ [165]. The modulated laser light exiting a high finesse optically stable cavity is phase-shifted with respect to the input light. The phase shift and amplitude-modulated intensity of the transmitted light are related to the modulation frequency and the ring-down time of the cavity using the equation $\tan (\varnothing)=-2 \pi f \tau$; the absorption spectrum is extracted from the magnitude of the phase shift [165-169]. This approach does not require fast detectors and other electronics and instead a lock-in amplifier may be used to determine the relative phase shift and the modulated intensity $[165,169]$.

Another promising mode toward the development of novel optoelectric materials, such as solar cells and light emitting diodes (LED), is polarization-dependent CRDS to study electron quantum confinement of nanostructure semiconductor materials $[170,171]$. 


\section{REFERENCES}

1. Rubahn, V.G.B.a.H.-G., Surfaces and Interfaces, in Optics and Spectroscopy at Surfaces and Interfaces. 2005, willy-VCH p. 5-56.

2. Zaera, F., Surface chemistry at the liquid/solid interface. Surface Science, 2011. 605(13): p. 1141-1145.

3. Rouquerol, J. and F. Rouquerol, 4 -Adsorption at the Liquid-Solid Interface: Thermodynamics and Methodology, in Adsorption by Powders and Porous Solids (Second Edition), F. Rouquerol, et al., Editors. 2014, Academic Press: Oxford. p. 105-158.

4. Martin, L., et al., Force fields for simulating the interaction of surfaces with biological molecules. Interface Focus, 2016. 6(1): p. 20150045.

5. Limo, M.J., et al., Interactions between Metal Oxides and Biomolecules: from Fundamental Understanding to Applications. Chemical Reviews, 2018. 118(22): p. 11118-11193.

6. Levitin, G. and D.W. Hess, Surface Reactions in Microelectronics Process Technology. Annual Review of Chemical and Biomolecular Engineering, 2011. 2(1): p. 299-324.

7. Huang, W. and W.-X. Li, Surface and interface design for heterogeneous catalysis. Physical Chemistry Chemical Physics, 2019. 21(2): p. 523-536.

8. Zhang, J., et al., Interfacial electrochemical electron transfer in biology-Towards the level of the single molecule. FEBS Letters, 2012. 586(5): p. 526-535.

9. Ikeda, T. and K. Kano, An electrochemical approach to the studies of biological redox reactions and their applications to biosensors, bioreactors, and biofuel cells. Journal of Bioscience and Bioengineering, 2001. 92(1): p. 9-18.

10. Hill, S.J. and A.S. Fisher, Atomic Absorption, Methods and Instrumentation, in Encyclopedia of Spectroscopy and Spectrometry, J.C. Lindon, Editor. 1999, Elsevier: Oxford. p. 24-32.

11. Hollas, J.M., Modern Spectroscopy. 2004: Wiley.

12. van der Sneppen, L., et al., Improving the sensitivity of HPLC absorption detection by cavity ring-down spectroscopy in a liquid-only cavity. Analytica Chimica Acta, 2006. 558: p. 2-6.

13. Li, R., H.-P. Loock, and R.D. Oleschuk, Capillary Electrophoresis Absorption Detection Using Fiber-Loop Ring-Down Spectroscopy. Analytical Chemistry, 2006. 78(16): p. 5685-5692. 
14. van der Sneppen, L., et al., Evanescent-Wave Cavity Ring-Down Spectroscopy for Enhanced Detection of Surface Binding Under Flow Injection Analysis Conditions. Vol. 62. 2008. 649-54.15. Berden, G., R. Peeters, and G. Meijer, Cavity ringdown spectroscopy: Experimental schemes and applications. International Reviews in Physical Chemistry, 2000. 19(4): p. 565-607.

16. Jongma, R.T., et al., Trace gas detection with cavity ring down spectroscopy. Review of Scientific Instruments, 1995. 66(4): p. 2821-2828.

17. J. M. Herbelin, J.A.M., M. A. Kwok, R. H. Ueunten, D. S. Urevig, D. J. Spencer, and D. J. Benard and Sensitive measurement of photon lifetime and true reflectances in an optical cavity by a phase-shift method. Appl. Opt, 1980. 19(1): p. 144-147.

18. Dana Z. Anderson, J.C.F., and Carl S. Masser, Mirror reflectometer based on optical cavity decay time. Appl. Opt., 1984. 23(8): p. 1238-1245.

19. O'Keefe, A. and D.A.G. Deacon, Cavity ring-down optical spectrometer for absorption measurements using pulsed laser sources. Review of Scientific Instruments, 1988. 59(12): p. 2544-2551.

20. Haselberg, R., et al., Effectiveness of Charged Noncovalent Polymer Coatings against Protein Adsorption to Silica Surfaces Studied by Evanescent-Wave Cavity Ring-Down Spectroscopy and Capillary Electrophoresis. Analytical Chemistry, 2009. 81(24): p. 10172-10178.

21. Zalicki, P. and R.N. Zare, Cavity ring-down spectroscopy for quantitative absorption measurements. The Journal of Chemical Physics, 1995. 102(7): p. 27082717.

22. Yoo, Y.S., et al., High-resolution cavity ringdown spectroscopy with a FabryPerot etalon at the cavity output. Chemical Physics Letters, 2000. 330(5): p. 528534.

23. Lehmann, K.K. and D. Romanini, The superposition principle and cavity ringdown spectroscopy. The Journal of Chemical Physics, 1996. 105(23): p. 1026310277.

24. van Zee, R.D., J.T. Hodges, and J.P. Looney, Pulsed, single-mode cavity ringdown spectroscopy. Applied Optics, 1999. 38(18): p. 3951-3960.

25. van der Sneppen, L., et al., Liquid-Phase and Evanescent-Wave Cavity Ring-Down Spectroscopy in Analytical Chemistry. Annual Review of Analytical Chemistry, 2009. 2(1): p. 13-35.

26. Hallock, A.J., E.S.F. Berman, and R.N. Zare, Direct Monitoring of Absorption in Solution by Cavity Ring-Down Spectroscopy. Analytical Chemistry, 2002. 74(7): p. 1741-1743.

27. Hallock, A.J., E.S.F. Berman, and R.N. Zare, Ultratrace Kinetic Measurements of the Reduction of Methylene Blue. Journal of the American Chemical Society, 2003. 125(5): p. 1158-1159.

28. Hallock, A.J., E.S.F. Berman, and R.N. Zare, Use of Broadband, Continuous-Wave Diode Lasers in Cavity Ring-Down Spectroscopy for Liquid Samples. Applied Spectroscopy, 2003. 57(5): p. 571-573.

29. Alexander, A.J., Flowing Liquid-Sheet Jet for Cavity Ring-Down Absorption Measurements. Analytical Chemistry, 2006. 78(15): p. 5597-5600. 
30. Fiedler, S.E., A. Hese, and A.A. Ruth, Incoherent broad-band cavity-enhanced absorption spectroscopy of liquids. Review of Scientific Instruments, 2005. 76(2): p. 023107.

31. $\mathrm{Xu}, \mathrm{S} ., \mathrm{G}$. Sha, and J. Xie, Cavity ring-down spectroscopy in the liquid phase. Review of Scientific Instruments, 2002. 73: p. 255-258.

32. Alexander, A.J., Reaction kinetics of nitrate radicals with terpenes in solution studied by cavity ring-down spectroscopy. Chemical Physics Letters, 2004. 393(1): p. 138-142.

33. Engeln, R., et al., Polarization dependent cavity ring down spectroscopy. The Journal of Chemical Physics, 1997. 107(12): p. 4458-4467.

34. Engeln, R., et al., Cavity ring down spectroscopy on solid C60. The Journal of Chemical Physics, 1999. 110(5): p. 2732-2733.

35. Egashira, K., A. Terasaki, and T. Kondow, Infrared spectra of organic monolayer films in a standing wave measured by photon-trap spectroscopy. The Journal of Chemical Physics, 2007. 126(22): p. 221102.

36. Logunov, S.L., Cavity ringdown detection of losses in thin films in the telecommunication wavelength window. Applied Optics, 2001. 40(9): p. 15701573.

37. Marcus, G.A. and H.A. Schwettman, Cavity ringdown spectroscopy of thin films in the mid-infrared. Applied Optics, 2002. 41(24): p. 5167-5171.

38. Pipino, A.C.R., J.W. Hudgens, and R.E. Huie, Evanescent wave cavity ring-down spectroscopy for probing surface processes. Chemical Physics Letters, 1997. 280(1): p. 104-112.

39. Hannon, T.E., S. Chah, and R.N. Zare, Evanescent-Wave Cavity Ring-Down Investigation of Polymer/Solvent Interactions. The Journal of Physical Chemistry B, 2005. 109(15): p. 7435-7442.

40. van der Sneppen, L., et al., Evanescent-wave cavity ring-down detection of cytochrome c on surface-modified prisms. Sensors and Actuators B: Chemical, 2009. 139(2): p. 505-510.

41. Schnippering, M., et al., Evanescent wave broadband cavity enhanced absorption spectroscopy using supercontinuum radiation: A new probe of electrochemical processes. Electrochemistry Communications, 2008. 10(12): p. 1827-1830.

42. Schnippering, M., et al., Evanescent wave cavity-based spectroscopic techniques as probes of interfacial processes. Chemical Society Reviews, 2011. 40(1): p. 207220.

43. Harrick, N.J., Internal reflection spectroscopy. Harrick Scientific Corporation, New York, 1987.

44. Dunphy, D.R., et al., The Electroactive Integrated Optical Waveguide: Ultrasensitive Spectroelectrochemistry of Submonolayer Adsorbates. Analytical Chemistry, 1997. 69(15): p. 3086-3094.

45. Doherty, W.J., et al., Potential-Modulated, Attenuated Total Reflectance Spectroscopy of Poly(3,4-ethylenedioxythiophene) and Poly(3,4ethylenedioxythiophene Methanol) Copolymer Films on Indium-Tin Oxide. The Journal of Physical Chemistry B, 2006. 110(10): p. 4900-4907. 
46. Matsuda, N., et al., Spectroelectrochemical studies on surface immobilized cytochrome c on ITO electrode by slab optical waveguide spectroscopy. Thin Solid Films, 2003. 438-439: p. 403-406.

47. Han, X. and S.B. Mendes, Optical Impedance Spectroscopy with Single-Mode Electro-Active-Integrated Optical Waveguides. Analytical Chemistry, 2014. 86(3): p. 1468-1477.

48. Powell, H.V., et al., Evanescent Wave Cavity Ring-Down Spectroscopy as a Probe of Interfacial Adsorption: Interaction of Tris(2,2'-bipyridine)ruthenium(II) with Silica Surfaces and Polyelectrolyte Films. Langmuir, 2009. 25(1): p. 248-255.

49. Mazurenka, M., et al., Evanescent Wave Cavity Ring-Down Spectroscopy in a ThinLayer Electrochemical Cell. Analytical Chemistry, 2006. 78(19): p. 6833-6839.

50. Powell, H.V., et al., Probing Redox Reactions of Immobilized Cytochrome c Using Evanescent Wave Cavity Ring-Down Spectroscopy in a Thin-Layer Electrochemical Cell. ChemPhysChem, 2010. 11(13): p. 2985-2991.

51. Pipino, A.C.R., Ultrasensitive Surface Spectroscopy with a Miniature Optical Resonator. Physical Review Letters, 1999. 83(15): p. 3093-3096.

52. Li, F. and R.N. Zare, Molecular Orientation Study of Methylene Blue at an Air/Fused-Silica Interface Using Evanescent-Wave Cavity Ring-Down Spectroscopy. The Journal of Physical Chemistry B, 2005. 109(8): p. 3330-3333.

53. Shaw, A.M., et al., Adsorption of Crystal Violet to the Silica-Water Interface Monitored by Evanescent Wave Cavity Ring-Down Spectroscopy. The Journal of Physical Chemistry B, 2003. 107(29): p. 7070-7075.

54. Fan, H.-F., et al., Characterization of Two Types of Silanol Groups on Fused-Silica Surfaces Using Evanescent-Wave Cavity Ring-Down Spectroscopy. Analytical Chemistry, 2007. 79(10): p. 3654-3661.

55. O'Reilly, J.P., et al., Interfacial pH at an Isolated Silica-Water Surface. Journal of the American Chemical Society, 2005. 127(6): p. 1632-1633.

56. Fan, H.-F., C.-Y. Hung, and K.-C. Lin, Molecular Adsorption at Silica/CH3CN Interface Probed by Using Evanescent Wave Cavity Ring-Down Absorption Spectroscopy: Determination of Thermodynamic Properties. Analytical Chemistry, 2006. 78(11): p. 3583-3590.

57. Chen, M.-S., H.-F. Fan, and K.-C. Lin, Kinetic and Thermodynamic Investigation of Rhodamine B Adsorption at Solid/Solvent Interfaces by Use of Evanescent-Wave Cavity Ring-Down Spectroscopy. Analytical Chemistry, 2010. 82(3): p. 868-877.

58. Gomes, I., et al., Controlled adsorption of cytochrome $c$ to nanostructured gold surfaces. Journal of Nanoparticle Research, 2012. 14(12): p. 1321.

59. Araci, I.E., et al., Highly sensitive spectroscopic detection of heme-protein submonolayer films by channel integrated optical waveguide. Optics Express, 2007. 15(9): p. 5595-5603.

60. Turner, A., I. Karube, and G.S. Wilson, eds. Biosensors : Fundamentals and Applications. 1 ed. 1987, Oxford University Press: Oxford, New York. 770.

61. Sallez, Y., P. Bianco, and E. Lojou, Electrochemical behavior of c-type cytochromes at clay-modified carbon electrodes: a model for the interaction between proteins and soils. Journal of Electroanalytical Chemistry, 2000. 493(1): p. 37-49. 
62. Quiquampoix, H., et al., Interpretation of the $p H$ dependence of protein adsorption on clay mineral surfaces and its relevance to the understanding of extracellular enzyme activity in soil. Colloids and Surfaces A: Physicochemical and Engineering Aspects, 1993. 75: p. 85-93.

63. Wang, X., et al., A new approach to detecting biologically active substances with evanescent-wave cavity ring-down spectroscopy. Vol. 467. 2008. 9-13.

64. Everest, M.A., et al., Hemoglobin Adsorption to Silica Monitored with Polarization-Dependent Evanescent-Wave Cavity Ring-Down Spectroscopy. The Journal of Physical Chemistry B, 2006. 110(39): p. 19461-19468.

65. Martin, W., et al., Hemoglobin adsorption isotherm at the silica-water interface with evanescent wave cavity ring-down spectroscopy. 2005. 10 \%J Journal of Biomedical Optics(2): p. 024025.

66. Zhang, M., et al., Kinetics of Porphyrin Adsorption and DNA-Assisted Desorption at the Silica-Water Interface. Langmuir, 2010. 26(6): p. 4004-4012.

67. Schnippering, M., et al., Surface Assembly and Redox Dissolution of Silver Nanoparticles Monitored by Evanescent Wave Cavity Ring-Down Spectroscopy. The Journal of Physical Chemistry C, 2008. 112(39): p. 15274-15280.

68. Bechtel, K.L., et al., Moving beyond Traditional UV-Visible Absorption Detection: Cavity Ring-Down Spectroscopy for HPLC. Analytical Chemistry, 2005. 77(4): p. 1177-1182.

69. van der Sneppen, L., et al., Cavity ring-down spectroscopy for detection in liquid chromatography at $U V$ wavelengths using standard cuvettes in a normal incidence geometry. Journal of chromatography. A, 2007. 1148: p. 184-8.

70. Engeln, R., et al., Detection of $\mathrm{CH}$ in an expanding argon/acetylene plasma using cavity ring down absorption spectroscopy. Chemical Physics Letters, 1999. 310(5): p. 405-410.

71. Scherer, J.J., et al., Cavity Ringdown Laser Absorption Spectroscopy: History, Development, and Application to Pulsed Molecular Beams. Chemical Reviews, 1997. 97(1): p. 25-52.

72. Technologies, A., https://amplitude-laser.com/produit/surelite-i-ii-iiil. Surelite I, II, III.

73. Sprague, M.K., Cavity Ringdown Spectroscopy, Kinetics, and Quantum Chemistry of Atmospherically Relevant Reactions. Dissertation (Ph.D.). California Institute of Technology, 2012: p. 34-36.

74. Spence, T.G., et al., A laser-locked cavity ring-down spectrometer employing an analog detection scheme. Review of Scientific Instruments, 2000. 71(2): p. 347353.

75. http://www.ic72.com/pdf file/h/247263.pdf.

76. $\quad$ http://www.crd-optics.com/crd-mirrors-vis.html.

77. Koechner, W., Optical Resonator, in Solid-State Laser Engineering, W. Koechner, Editor. 2006, Springer New York: New York, NY. p. 210-299.

78. Bahaa E. A. Saleh, M.C.T., Fundamentals of Photonics, Resonator Optics. John Wiley \& Sons, Copyright (C) 1991 p. 310-341.

79. Wang, S., et al., Cavity-enhanced absorption spectroscopy with a ps-pulsed UV laser for sensitive, high-speed measurements in a shock tube. Optics Express, 2016. 24(1): p. 308-318. 
80. Collinson, M. and E.F. Bowden, Chronoabsorptometric determination of adsorption isotherms for cytochrome $c$ on tin oxide electrodes. Langmuir, 1992. 8(10): p. 2552-2559.

81. Mousty, C., Sensors and biosensors based on clay-modified electrodes - New trends. Vol. 27. 2004. 159-177.

82. Dickinson, E., Mixed proteinaceous emulsifiers: review of competitive protein adsorption and the relationship to food colloid stabilization. Food Hydrocolloids, 1986. 1(1): p. 3-23.

83. Han, X. and S.B. Mendes, Spectroelectrochemical properties of ultra-thin indium tin oxide films under electric potential modulation. Thin Solid Films, 2016. 603: p. 230-237.

84. Mendes, S., S. Scott Saavedra, and N. R. Armstrong, Broadband Spectroelectrochemical Interrogation of Molecular Thin Films by Single-Mode Electro-Active Integrated Optical Waveguides. 2009. p. 101-129.

85. Runge, A.F., S.B. Mendes, and S.S. Saavedra, Order Parameters and Orientation Distributions of Solution Adsorbed and Microcontact Printed Cytochrome $c$ Protein Films on Glass and ITO. The Journal of Physical Chemistry B, 2006. 110(13): p. 6732-6739.

86. Runge, A.F. and S.S. Saavedra, Comparison of Microcontact-Printed and SolutionAdsorbed Cytochrome c Films on Indium Tin Oxide Electrodes. Langmuir, 2003. 19(22): p. 9418-9424.

87. Kim, J., et al., Formation, structure, and reactivity of amino-terminated organic films on silicon substrates. Journal of Colloid and Interface Science, 2009. 329(1): p. 114-119.

88. Ghithan, J.H., et al., Influenza virus immunosensor with an electro-active optical waveguide under potential modulation. Optics Letters, 2017. 42(7): p. 1205-1208.

89. Saha, K., F. Bender, and E. Gizeli, Comparative Study of IgG Binding to Proteins $G$ and $A$ : Nonequilibrium Kinetic and Binding Constant Determination with the Acoustic Waveguide Device. Analytical Chemistry, 2003. 75(4): p. 835-842.

90. Karlsson, R., A. Michaelsson, and L. Mattsson, Kinetic analysis of monoclonal antibody-antigen interactions with a new biosensor based analytical system. Journal of Immunological Methods, 1991. 145(1): p. 229-240.

91. Qi, Z.-m., et al., A Kinetic Study of Cytochrome c Adsorption to Hydrophilic Glass by Broad-Band, Time-Resolved Optical Waveguide Spectroscopy. The Journal of Physical Chemistry B, 2003. 107(28): p. 6873-6875.

92. Fedurco, M., Redox reactions of heme-containing metalloproteins: dynamic effects of self-assembled monolayers on thermodynamics and kinetics of cytochrome $c$ electron-transfer reactions. Coordination Chemistry Reviews, 2000. 209(1): p. 263-331.

93. Schreurs, J., et al., Electrochemical behaviour of horse-heart cytochrome-c. Recueil des Travaux Chimiques des Pays-Bas, 1984. 103(9): p. 263-269.

94. Rawat, S.P. and D.P. Khali, Enthalpy-entropy compensation during sorption of water in wood. Journal of Applied Polymer Science, 1996. 60(5): p. 787-790.

95. Mendes, S., Spectroelectrochemical properties of ultra-thin indium tin oxide films under electric potential modulation. Vol. 603. 2016. 230-237. 
96. Peng, C., et al., Molecular simulations of cytochrome c adsorption on positively charged surfaces: the influence of anion type and concentration. Physical Chemistry Chemical Physics, 2016. 18(15): p. 9979-9989.

97. Jarocka, U., et al., Electrochemical immunosensor for detection of antibodies against influenza A virus H5N1 in hen serum. Biosensors and Bioelectronics, 2014. 55: p. 301-306.

98. Siuzdak, K., et al., Biomolecular influenza virus detection based on the electrochemical impedance spectroscopy using the nanocrystalline boron-doped diamond electrodes with covalently bound antibodies. Sensors and Actuators B: Chemical, 2019. 280: p. 263-271.

99. Zhou, Y.-M., et al., An amperometric immunosensor based on Nafion-modified electrode for the determination of Schistosoma japonicum antibody. Sensors and Actuators B: Chemical, 2003. 89(3): p. 292-298.

100. Beam, B.M., N.R. Armstrong, and S.B. Mendes, An electroactive fiber optic chip for spectroelectrochemical characterization of ultra-thin redox-active films. Analyst, 2009. 134(3): p. 454-459.

101. Qatamin, A.H., et al., Detection of influenza virus by electrochemical surface plasmon resonance under potential modulation. Applied Optics, 2019. 58(11): p. 2839-2844.

102. Wang, J., F. Wang, and S. Dong, Methylene blue as an indicator for sensitive electrochemical detection of adenosine based on aptamer switch. Journal of Electroanalytical Chemistry, 2009. 626(1): p. 1-5.

103. Feng, K., et al., Label-free electrochemical detection of nanomolar adenosine based on target-induced aptamer displacement. Electrochemistry Communications, 2008. 10(4): p. 531-535.

104. Imai, K., et al., Simultaneous Multiselective Spectroelectrochemical Fiber-Optic Sensor: Demonstration of the Concept Using Methylene Blue and Ferrocyanide. Analytical Chemistry, 2015. 87(4): p. 2375-2382.

105. Du, X., et al., The Trace Detection of Nitrite Ions Using Neutral Red Functionalized SH- $\beta$-Cyclodextrin@Au Nanoparticles. Sensors,2018.18(3).

106. Tran, C.T.K., et al., Determination of low level nitrate/nitrite contamination using SERS-active Ag/ITO substrates coupled to a self-designed Raman spectroscopy system. Journal of Science: Advanced Materials and Devices, 2017. 2(2): p. 172177.

107. Beamonte, E., et al., A statistical study of the quality of surface water intended for human consumption near Valencia (Spain). Journal of Environmental Management, 2007. 83(3): p. 307-314.

108. Battas, A., et al., Adsorption Study for the Removal of Nitrate from Water Using Local Clay. The Scientific World Journal, 2019. 2019: p. 9529618.

109. Electrocoagulation. Kinetic Modelling of Nitrate Removal from Aqueous Solution during Electrocoagulation. 2013.

110. Zhang, Y., et al., Template-assisted preparation of Au nanowires and their application in nitrite ions sensing. Journal of Electroanalytical Chemistry, 2016. 773: p. 1-6. 
111. Nouroozi, S. and R. Mirshafian, Flow injection kinetic spectrophotometric method for the determination of trace amounts of nitrite. Talanta, 2009. 79(4): p. 11491153.

112. Doane, T.A. and W.R. Horwáth, Spectrophotometric Determination of Nitrate with a Single Reagent. Analytical Letters, 2003. 36(12): p. 2713-2722.

113. Tirumalesh, K., Simultaneous determination of bromide and nitrate in contaminated waters by ion chromatography using amperometry and absorbance detectors. Talanta, 2008. 74(5): p. 1428-1434.

114. Cherian, T. and B. Narayana, A new system for the spectrophotometric determination of trace amounts of nitrite in environmental samples \%J Journal of the Brazilian Chemical Society. 2006. 17: p. 577-581.

115. Yang, L., et al., Characteristics of Nitrate Removal from Aqueous Solution by Modified Steel Slag. Water, 2017. 9(10).

116. Barzegar, M., M.F. Mousavi, and A. Nemati, Kinetic spectrophotometric determination of trace amounts of nitrite by its reaction with molybdosilicic acid blue. Microchemical Journal, 2000. 65(2): p. 159-163.

117. Mousavi, M.F., A. Jabbari, and S. Nouroozi, A sensitive flow-injection method for determination of trace amounts of nitrite. Talanta, 1998. 45(6): p. 1247-1253.

118. Guan, F., H. Wu, and Y. Luo, Sensitive and selective method for direct determination of nitrite and nitrate by high-performance capillary electrophoresis. Journal of Chromatography A, 1996. 719(2): p. 427-433.

119. Kalaruban, M., et al., Enhanced removal of nitrate in an integrated electrochemical-adsorption system. Separation and Purification Technology, 2017. 189: p. 260-266.

120. Ojani, R., J.B. Raoof, and E. Zarei, Electrocatalytic reduction of nitrite using ferricyanide; Application for its simple and selective determination. Electrochimica Acta - ELECTROCHIM ACTA, 2006. 52: p. 753-759.

121. Chen, J., et al., Highly sensitive and selective detection of nitrite ions using Fe3O4@SiO2/Au magnetic nanoparticles by surface-enhanced Raman spectroscopy. Biosensors and Bioelectronics, 2016. 85: p. 726-733.

122. Du, X., S. Ye, and D. Dong, Rapid Determination of Nitrite in Drinking Water Using Ion-Exchange-Enhanced Infrared Spectroscopy. 2018.

123. Büldt, A. and U. Karst, Determination of Nitrite in Waters by Microplate Fluorescence Spectroscopy and HPLC with Fluorescence Detection. Analytical Chemistry, 1999. 71(15): p. 3003-3007.

124. Gürkan, R., H.İ. Ulusoy, and M. Akçay, A New Analytical Method for the Kinetic Spectrophotometric Determination of Trace Amounts of Nitrite in Some Environmental Water Samples. Eurasian Journal of Analytical Chemistry, 2010. 5(1): p. 16-31.

125. Li, Z., et al., Highly sensitive and selective method for detection of trace amounts of nitrite in aquaculture water by SERRS coupled with diazo reaction. Sensors and Actuators B: Chemical, 2019. 297: p. 126757.

126. Kim, J.B., et al., Wrinkles and deep folds as photonic structures in photovoltaics. Nature Photonics, 2012. 6(5): p. 327-332.

127. Tang, C.W., Two-layer organic photovoltaic cell. Applied Physics Letters, 1986. 48(2): p. 183-185. 
128. Huang, M., et al., Characterisation and Optimisation of Indium Tin Oxide Films Deposited by Pulsed DC Magnetron Sputtering for Heterojunction Silicon Wafer Solar Cell Applications. Energy Procedia, 2013. 33: p. 91-98.

129. Wu, C.C., et al., Surface modification of indium tin oxide by plasma treatment: An effective method to improve the efficiency, brightness, and reliability of organic light emitting devices. Applied Physics Letters, 1997. 70(11): p. 1348-1350.

130. Jonda, C., et al., Surface roughness effects and their influence on the degradation of organic light emitting devices. 2004(22): p. 5645-5651.

131. Choi, J.H., et al., Design and characterization of Ga-doped indium tin oxide films for pixel electrode in liquid crystal display. Thin Solid Films, 2013: p. 141-146.

132. Betz, U., et al., Thin films engineering of indium tin oxide: Large area flat panel displays application. Surface \& Coatings Technology, 2006. 200(20-21): p. 57515759.

133. Ge, C., et al., Voltammetric and waveguide spectroelectrochemical characterization of ultrathin poly(aniline)/poly(acrylic acid) films self-assembled on indium-tin oxide. Talanta, 2005. 65(5): p. 1126-1131.

134. Lin, H.-C., et al., Electron-Transfer Processes in Zinc Phthalocyanine-Phosphonic Acid Monolayers on ITO: Characterization of Orientation and Charge-Transfer Kinetics by Waveguide Spectroelectrochemistry. The Journal of Physical Chemistry Letters, 2012. 3(9): p. 1154-1158.

135. Wu, W.-F. and B.-S. Chiou, Deposition of indium tin oxide films on polycarbonate substrates by radio-frequency magnetron sputtering. Thin Solid Films, 1997. 298(1): p. 221-227.

136. Choi, S.K. and J.I. Lee, Effect of film density on electrical properties of indium tin oxide films deposited by dc magnetron reactive sputtering. Journal of Vacuum Science \& Technology A, 2001. 19(5): p. 2043-2047.

137. Park, Y.-C., et al., ITO thin films deposited at different oxygen flow rates on Si(100) using the PEMOCVD method. Surface and Coatings Technology, 2002. 161(1): p. 62-69.

138. Kim, Y.-S., et al., Effect of substrate temperature on the bonded states of indium tin oxide thin films deposited by plasma enhanced chemical vapor deposition. Thin Solid Films, 2003. 426(1): p. 124-131.

139. Zhou, Y., et al., Inverted organic solar cells with ITO electrodes modified with an ultrathin Al2O3 buffer layer deposited by atomic layer deposition. Journal of Materials Chemistry, 2010. 20(29): p. 6189-6194.

140. Elam, J.W., et al., Atomic Layer Deposition of Indium Tin Oxide Thin Films Using Nonhalogenated Precursors. The Journal of Physical Chemistry C, 2008. 112(6): p. 1938-1945.

141. Prepelita, P., et al., Rapid thermal annealing for high-quality ITO thin films deposited by radio-frequency magnetron sputtering. Beilstein Journal of Nanotechnology, 2019. 10: p. 1511-1522.

142. $\mathrm{Xu}, \mathrm{Z}$., et al., Influence of thermal annealing on electrical and optical properties of indium tin oxide thin films. Materials Science in Semiconductor Processing, 2014. 26: p. 588-592. 
143. Sharma, G., et al., Optical, electrical and photovoltaic properties of thermally annealed PPHT:DDE blend thin films. Journal of Physics and Chemistry of Solids, 2008. 69: p. 2639-2651.

144. Hammad, T.M., Effect of annealing on electrical, structural, and optical properties of sol-gel ITO thin films. physica status solidi (a), 2009. 206(9): p. 2128-2132.

145. Baía, I., et al., Influence of the process parameters on structural and electrical properties of r.f. magnetron sputtering ITO films. Thin Solid Films, 2001. 383(1): p. 244-247.

146. Shin, J.-W. and W.-J. Cho, Microwave Annealing Effects of Indium-Tin-Oxide Thin Films: Comparison with Conventional Annealing Methods. physica status solidi (a), 2018. 215(20): p. 1700975.

147. Van Slyke, S.A., C.H. Chen, and C.W. Tang, Organic electroluminescent devices with improved stability. Applied Physics Letters, 1996. 69(15): p. 2160-2162.

148. Kim, J.S., R.H. Friend, and F. Cacialli, Surface energy and polarity of treated indium-tin-oxide anodes for polymer light-emitting diodes studied by contactangle measurements. Journal of Applied Physics, 1999. 86(5): p. 2774-2778.

149. Kim, B.-S., et al., UV-Ozone Surface Treatment of Indium-Tin-Oxide in Organic Light Emitting Diodes. Journal of The Korean Physical Society - J KOREAN PHYS SOC, 2007. 50.

150. Aleksandrova, M., et al., Influence of UV Treatment on the Electro-optical Properties of Indium Tin Oxide Films Used in Flexible Displays. 2012.

151. Kim, S., et al., Effect of ultraviolet-ozone treatment of indium-tin-oxide on electrical properties of organic light emitting diodes. Journal of Applied Physics, 2004. 95: p. 2560-2563.

152. So, S.K., et al., Surface preparation and characterization of indium tin oxide substrates for organic electroluminescent devices. 1999(4): p. 447-450.

153. Yamashita, D., A. Ishizaki, and T. Yamamoto, $<i>$ In Situ $</ i>$ Measurements of Work Function of Indium Tin Oxide after UV/Ozone Treatment. MATERIALS TRANSACTIONS, 2015. 56(9): p. 1445-1447.

154. Lo, M.-F., et al., Direct Threat of a UV-Ozone Treated Indium-Tin-Oxide Substrate to the Stabilities of Common Organic Semiconductors. Advanced Functional Materials, 2013. 23(13): p. 1718-1723.

155. Herbelin, J.M., et al., Sensitive measurement of photon lifetime and true reflectances in an optical cavity by a phase-shift method. Appl Opt, 1980. 19(1): p. 144-7.

156. Song, W., et al., Angle dependent X-ray photoemission study on UV-ozone treatments of indium tin oxide. Applied Surface Science, 2001. 177(3): p. 158-164.

157. Artorn, P., M. Horprathum, and L. Pichet, Influence of oxygen flow rate on properties of indium tin oxide thin films prepared by ion-assisted electron beam evaporation. Songklanakarin Journal of Science and Technology, 2009. 31.

158. Meng, L., et al., Study of Indium Tin Oxide Thin Films Deposited on Acrylics Substrates by Ion Beam Assisted Deposition Technique. Journal of nanoscience and nanotechnology, 2009. 9: p. 4151-5.

159. Santos, E., E.C. Burini Junior, and S. Wang, UV-Ozone Generation from Modified High Intensity Discharge Mercury Vapor Lamps for Treatment of Indium Tin Oxide Films. Ozone-science \& Engineering - OZONE-SCI ENG, 2012. 34: p. 129-135. 
160. Paldus, B. and A. Kachanov, A historical overview of cavity-enhanced methods. CANADIAN JOURNAL OF PHYSICS, 2005. 83: p. 975-999.

161. Romanini, D., et al., $C W$ cavity ring down spectroscopy. Chemical Physics Letters, 1997. 264(3): p. 316-322.

162. Li, Z., et al., Continuous-wave cavity ringdown spectroscopy based on the control of cavity reflection. Optics Express, 2013. 21(15): p. 17961-17971.

163. Shumyantseva, V.V., et al., Direct electron transfer between cytochrome P450scc and gold nanoparticles on screen-printed rhodium-graphite electrodes. Biosensors and Bioelectronics, 2005. 21(1): p. 217-222.

164. De, A., et al., Continuous wave external-cavity quantum cascade laser-based highresolution cavity ring-down spectrometer for ultrasensitive trace gas detection. Opt Lett, 2016. 41(9): p. 1949-52.

165. Hovde, C. and A.L. Gomez, Phase-shift feedback cavity ring-down spectroscopy. Appl Opt, 2015. 54(17): p. 5555-9.

166. van Helden, J.H., D.C. Schram, and R. Engeln, Phase-shift cavity ring-down spectroscopy to determine absolute line intensities. Chemical Physics Letters, 2004. 400(4): p. 320-325.

167. Dhiman, C., M. Khan, and M.N. Reddy, Phase-shift Cavity Ring-down Technique for Detection of NO2 in PPM Concentration. Defence science journal, 2014. 64: p. $426-430$.

168. Tong, Z., et al., Phase-Shift Fiber-Loop Ring-Down Spectroscopy. Analytical Chemistry, 2004. 76(22): p. 6594-6599.

169. Barnes, J.A. and H.-P. Loock, Application of coupled mode theory and coherent superposition theory to phase-shift measurements on optical microresonators. Nanotechnology, 2016. 27(42): p. 424004.

170. Maruska, H.P., F. Namavar, and N.M. Kalkhoran, Energy bands in quantum confined silicon light-emitting diodes. Applied Physics Letters, 1993. 63(1): p. 4547.

171. Liu, R., C. McCormick, and C. Bayram, Suppression of indium clustering and quantum confined stark effect of InGaN LED on Silicon (111), in Gallium Nitride Materials and Devices Xiv, H. Fujioka, H. Morkoc, and U.T. Schwarz, Editors. 2019, Spie-Int Soc Optical Engineering: Bellingham. 


\section{APPENDICES}

Appendix 1: Labview VI's used for CRDS electrochemistry data acquisition

Figure 1 shows the Labview sub-VI program responsible for fitting of the ring-down traces and extraction of the corresponding ring-down time. The averaged ring-down traces were used to calculate the ring down time, $\tau$, using a least-square algorithm fitting routine.

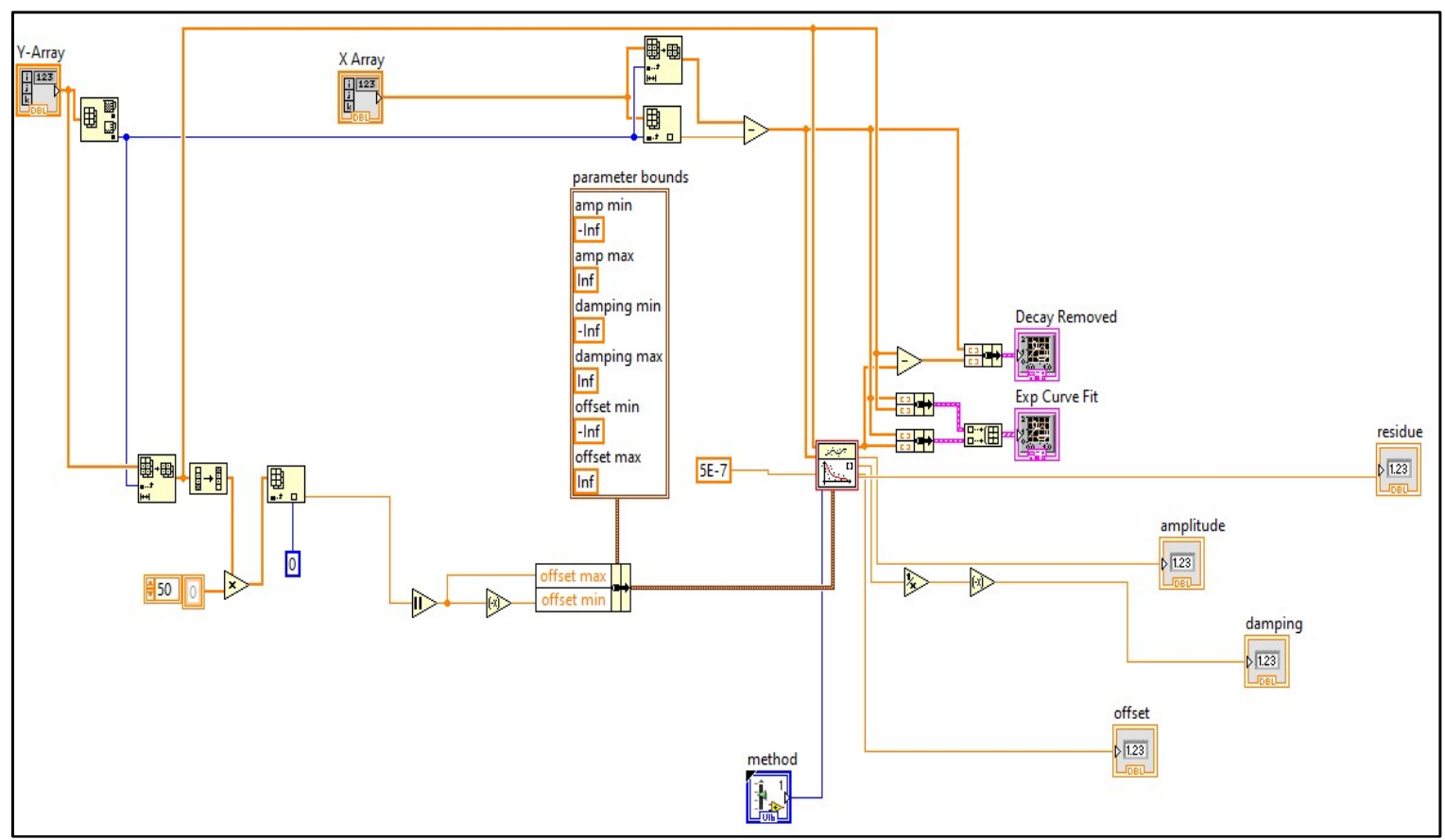

Figure 1: Screenshot block diagram of the exponential decay fit sub-VI that used to extract the ring-down time.

The LabVIEW VI was designed for the electrochemical measurements to be executed while the EW-CRDS program was running simultaneously. As shown in Figure 2(a), a sub-VI was used to read and set the electrochemical parameters, and controlling a triangular wave potential of CV scan. At the same time, a separate sub-VI was used to set 
the oscilloscope parameters, acquire and averaging of ring-down traces, and extract the ring-down times and corresponding electric potential data, as shown in Figure 2(b).

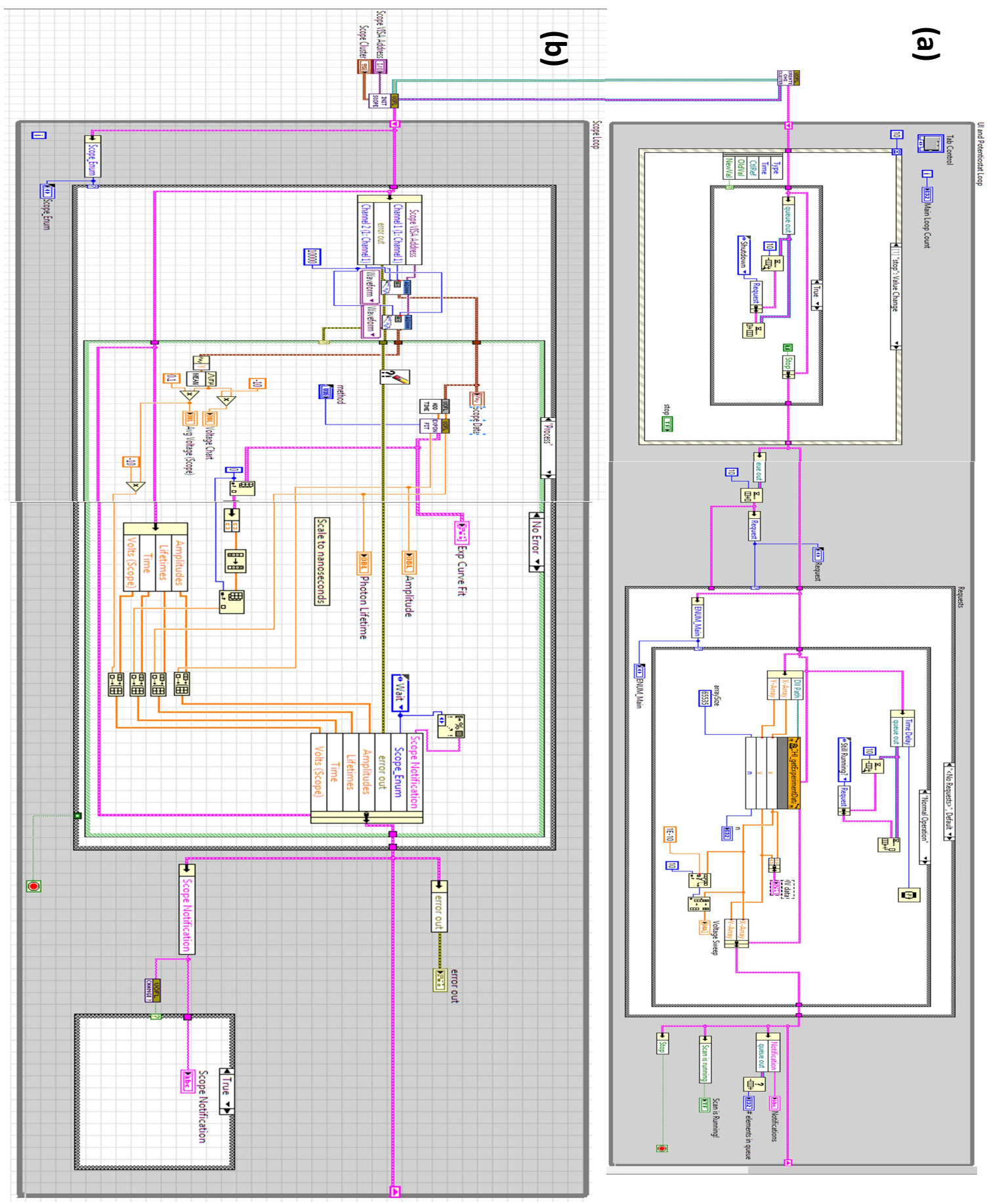

Figure 2: Block diagram of the VI used for the EW-CRDS spectroelectrochemistry data acquisition. (a) potentiostat sub-VI and (b) a sub-VI for extracting the ring-down times and the corresponding electric potential data. 
Appendix 2: Python program code for ring-down time calculation

import numpy as np

import matplotlib.pyplot as plt

from scipy.optimize import curve_fit

import pandas as pd

data_Signal_CRDS $=$ np.genfromtxt("cdrs_shadi.txt",skip_header $=2$, usecols= $(0,1))$

X_1 = data_Signal_CRDS[:,0]

$Y_{-} 1$ = data_Singnal_CRDS[:1]

plt.figure(figsize $=(10,5))$

plt.plot $\left(X_{-} 1, Y_{-} 1_{1}\right.$ '-r' $\left.^{\prime}\right)$

plt.ylabel('Intensity', fontsize=20)

plt.savefig('fignew_122.png')

plt.show()

def exponenial_func $(x, a, b, c)$ :

exponenial_func $=a * n p \cdot \exp \left(-\left(b^{*} x\right)\right)+c$ 
return exponenial_func

par,cov = curve_fit(exponenial_func, $\left.X_{-} 1, Y_{-} 1, p 0=[-25,1000000,0]\right)$

print par

$\mathrm{s}_{-} 1=1 / \mathrm{par}$

print s_ 1

$X_{\_}$new $=n p \cdot$ linspace $\left(n p \cdot a m i n\left(X_{-} 1\right), n p \cdot a m a x\left(X_{-} 1\right), \operatorname{len}\left(X_{-} 1\right) * 10\right)$

Y_fit $=$ exponenial_func $\left(x_{-}\right.$new, ${ }^{*}$ par $)$

plt.figure(figsize $=(15,10))$

plt.plot $\left(X_{-} 1, Y_{-} 1\right.$, 'o-y' $\left.^{\prime}\right)$

plt.plot(x_new,y_fit, '-k', Iw=2)

plt.xlabel('Time (s)', fontsize=20)

plt.ylabel('INENTSITY', fontsize=15)

plt.savefig('figure.png')

plt.show() 
Appendix 3: Cyt-C protein samples preparation

Horse heart cytochrome C with purity of $99.7 \%$ was purchased from Sigma Aldrich. A stock solution of Cyt-C at the oxidized stated was prepared by dissolving $\mathrm{Cyt}-\mathrm{C}$ bulk material in phosphate buffer saline (PBS), $\left(\mathrm{Na}_{2} \mathrm{HPO}_{4}, 50 \mathrm{mM}, \mathrm{pH} 7.02\right)$ solution. The protein concentration (c) was determined using a UV-Vis spectrophotometer for measuring the absorbance $(A)$ at $\lambda=409 \mathrm{~nm}$ and using Beer's law $\mathrm{c}=\mathrm{A} /(\epsilon \mathrm{b})$, where $\epsilon$ is the molar absorptivity of Cyt-C in its oxidized form at $409 \mathrm{~nm}\left(106,000 \mathrm{M}^{-1} \mathrm{~cm}^{-1}\right)$ and $b$ is the cuvette pathlength, $b=1 \mathrm{~cm}$. As needed, the stock solution was diluted in PBS solution to generate different protein concentrations in the $0.02-7 \mu \mathrm{M}$ range.

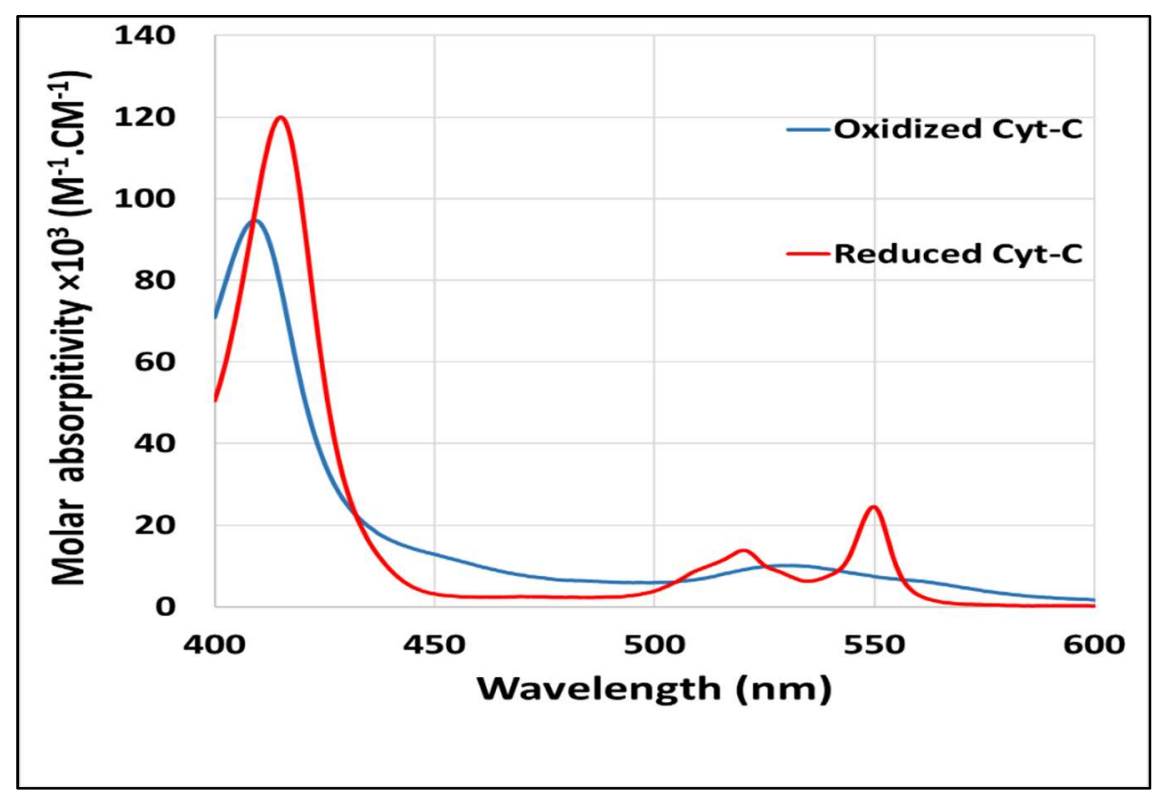

Figure 3: The molar absorptivity for the oxidized (blue) and reduced (red) forms of CytC protein of concentration $4.3 \mu \mathrm{M}$ in PBS buffer solution. The characteristics absorbance peaks (409 nm and $528 \mathrm{~nm}$ ) for the oxidized state, and (416 nm and $550 \mathrm{~nm})$ for the reduced state were observed. 
In order to obtain the spectrum of the reduced form, the same concentration of CytC protein $(\mathrm{c}=4.3 \mu \mathrm{M})$ was reduced chemically by using chemical reducing agent ascorbic acid $\left(\mathrm{C}_{6} \mathrm{H}_{8} \mathrm{O}_{6}\right)$ in the solution environment. The molar absorptivity for both oxidized and reduced states were calibrated, as shown in Figure 3. The extinction coefficients of the oxidized and reduced form of Cyt-C at $532 \mathrm{~nm}$ in PBS solution was found to be $\epsilon_{O x}=$ $10.05 \times 10^{3} \mathrm{M}^{-1} \mathrm{~cm}^{-1}$ and $7 \times 10^{3} \mathrm{M}^{-1} \mathrm{~cm}^{-1}$, respectively. 
Appendix 4: Binding Stability of the Cyt-C Ab on the APTES surface

Binding Stability of the Cyt-C Ab on the APTES Surface: The binding stability of the Cyt-C antibody to the APTES surface during the applied potential was confirmed by applying different values of an applied potential. First, as shown in Figure 4, the experiment was done under open circuit potential (OCP) until the adsorption reaches the plateau adsorption region, then $-0.4 \mathrm{~V}$ and $0.4 \mathrm{~V}$ potential was applied, respectively. The results indicate that the binding of negatively charged $\mathrm{Cyt}-\mathrm{C} \mathrm{Ab}$ is not affected by the applied potential, and the adsorption of positively charged Cyt-C protein is enhanced when negative values of the potential were applied.

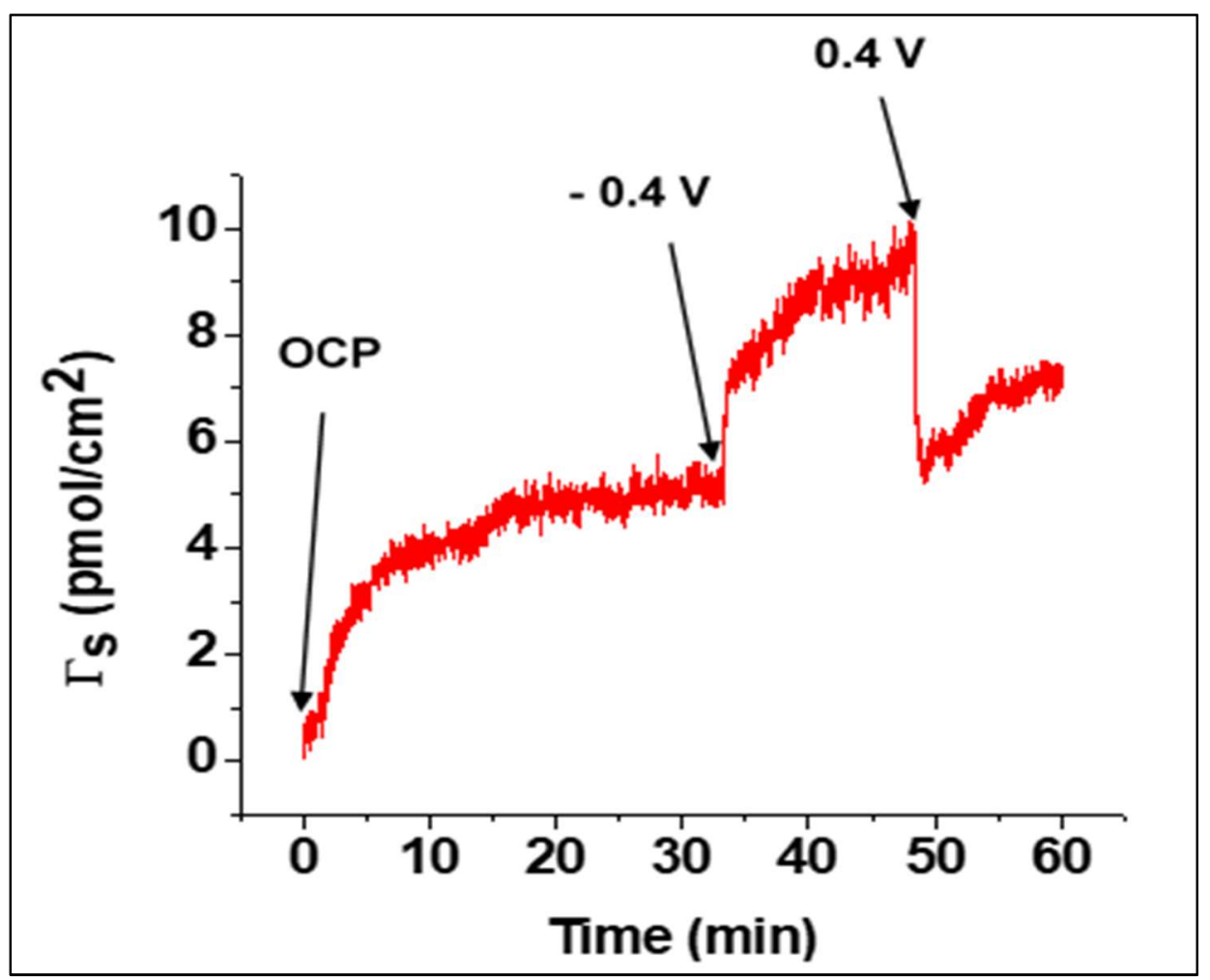

Figure 4: The surface coverage vs time of $500 \mathrm{nM}$ of Cyt-C adsorption on ITO/APTES/Cyt-C Ab for different value of applied potential (OCP, $-04 \mathrm{~V}$, and 0.4V). 
Appendix 5: Kinetic Adsorption of Cyt-C Protein onto the APTES and Antbody Surface at Open Potential

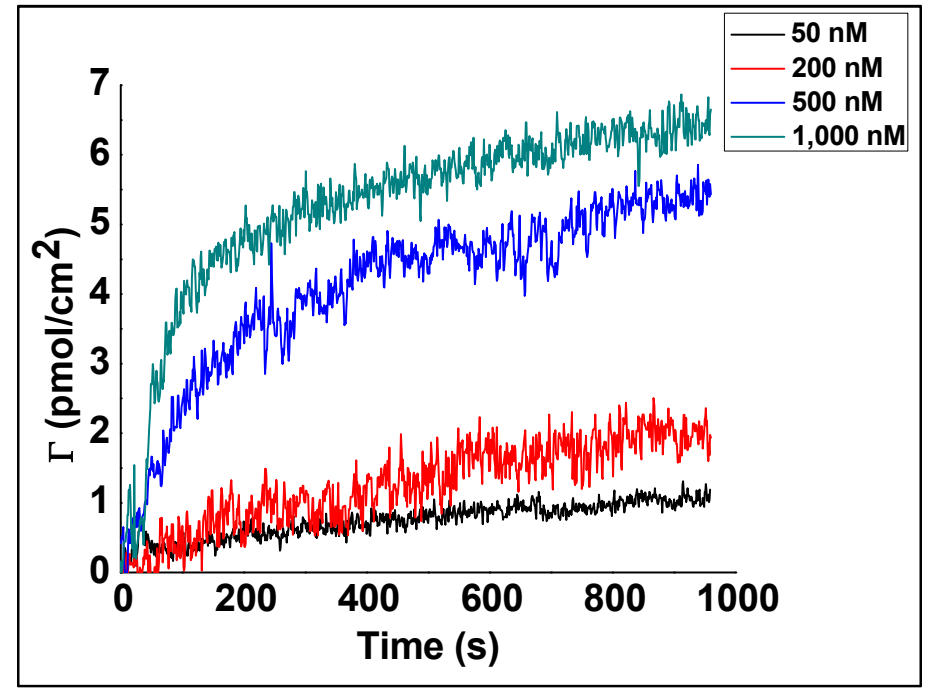

Figure 5: Surface coverage change of Cyt-C binding on the ITO/APTES measured by EW-CRDS platform for different concentrations in real-time.

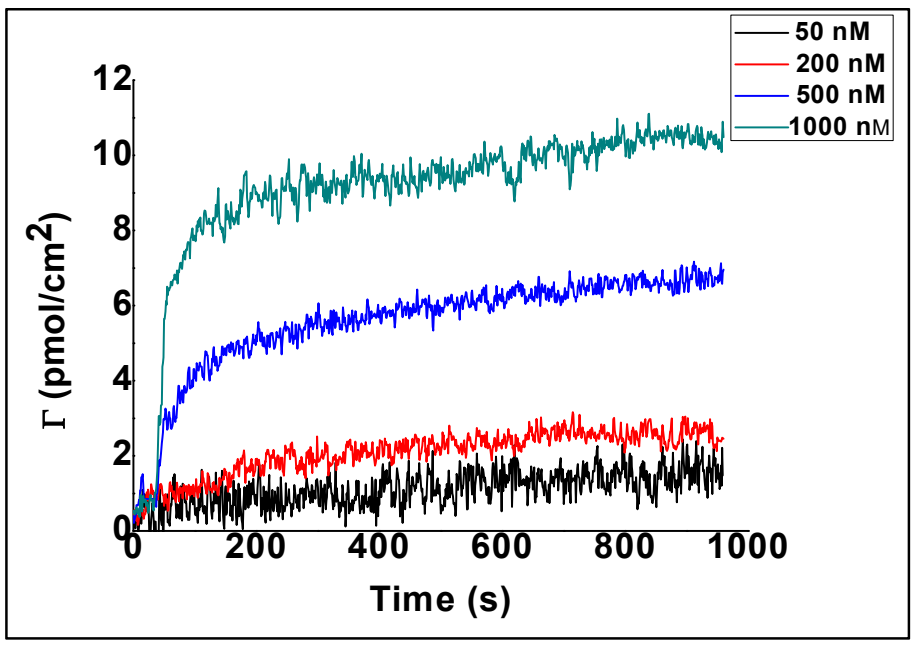

Figure 6: Surface coverage change of Cyt-C binding on the bare ITO/APTES/Cyt-C measured by EW-CRDS platform for different concentrations in real-time. 
Appendix 6: Kinetic Adsorption of Cyt-C Protein onto the APTES and Antibody Surface at Controlled Potential
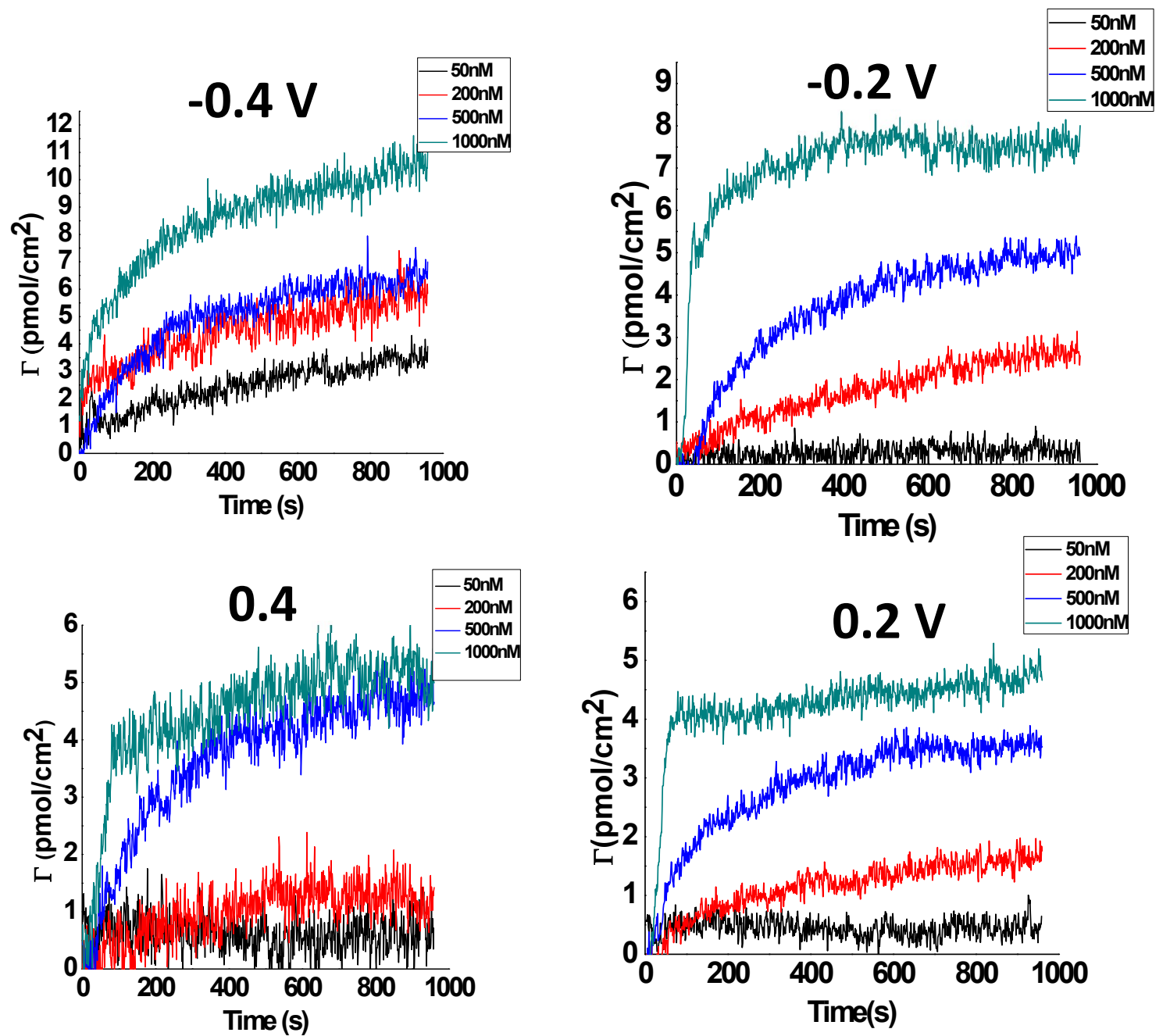

Figure 7: Surface coverage vs time for different concentrations of Cyt-C with different values of external electric potential on ITO/APTES surface. 

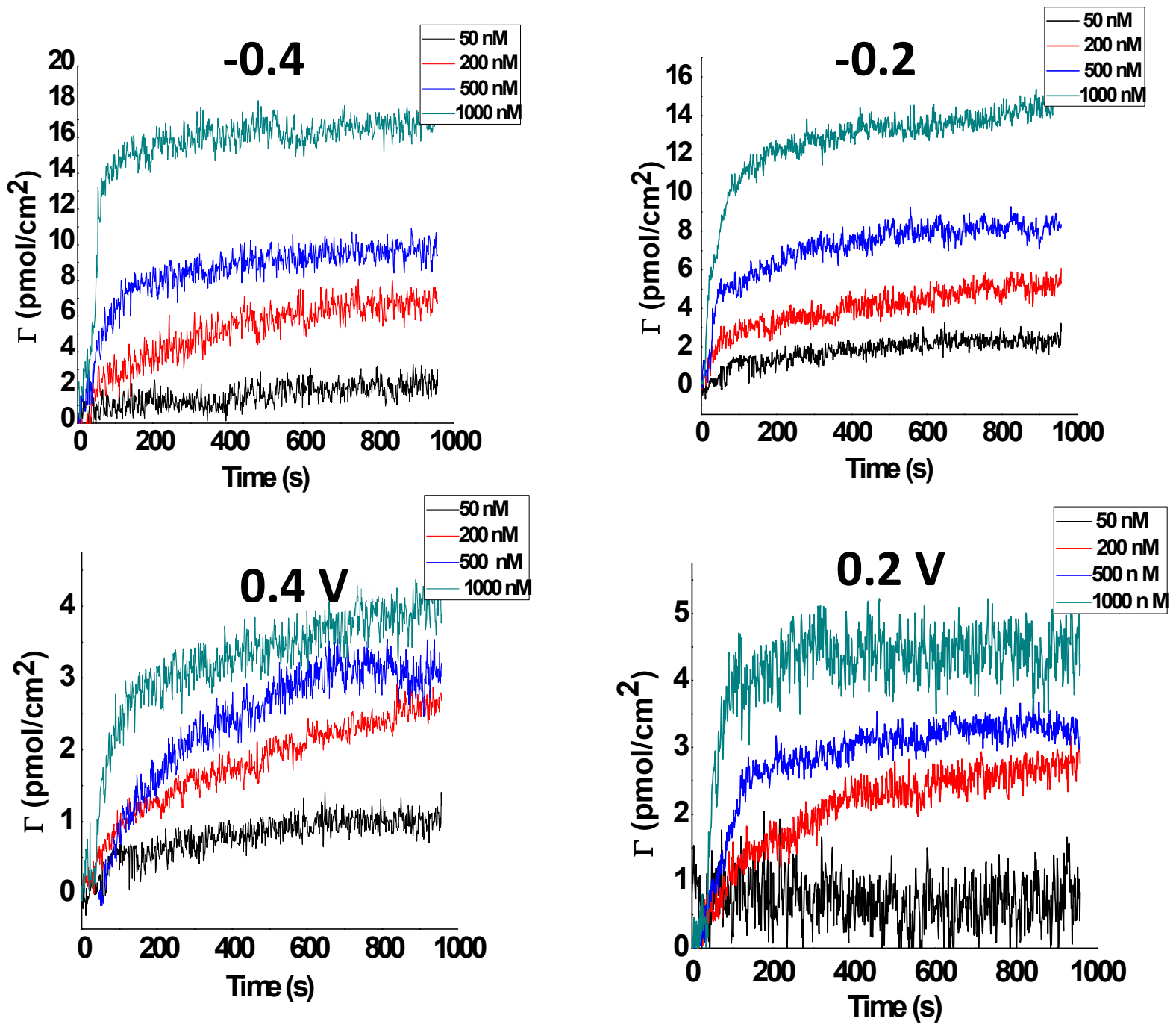

Figure 8: Surface coverage vs time for different concentrations of Cyt-C with different values of external electric potential on ITOAPTES/Cyt-C Ab surface. 
Appendix 7: MB-Labeled H5N1 secondary Ab concentration Calculations

The concentration of the MB-Labeled Ab was determine by using Beer's law and UV Vis spectrum of the conjugate solution as following:

Antibody concentration $=\frac{\left(A_{280}-\left(A_{610} \times 0.28\right)\right) \times \text { dilutionfactor }}{203000}$

where $A_{610}$ is the absorbance value at $610 \mathrm{~nm}$ (which is associated to the maximum absorbance of the $\mathrm{MB}$ ester dye at $610 \mathrm{~nm}), A_{280}$ is the maximum absorbance of $\mathrm{Ab}$ protein, 0.28 is correction factor to account for absorption of the dye at $280 \mathrm{~nm}$, and $203000 \mathrm{~cm}^{-1} \mathrm{M}^{-1}$ is the molar extinction coefficient of a typical $\mathrm{IgG}$ antibody.

The concentration of MB ester dye can be measured by:

MB ester dye concentration $=\frac{A_{610} \times \text { dilution factor }}{26000}$

where 26000 is the molar extinction coefficient MB dye at $610 \mathrm{~nm}$.

Degree of labelling $=\frac{\text { antibody concentration }}{\text { dye concentration }}$ 
Appendix 8 (a): Mathematica program code for optical constants calculations of ITO thin-film

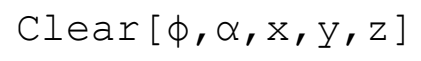
[1] ] ;

RpExp=Import ["C: \Users \Shadi \Desktop \math\inputSA.xlsx", "xls $x "][[2]]$;

TpExp=Import $[$ "C: \Users $\backslash$ Shadi $\backslash$ Desktop $\backslash$ math $\backslash$ inputSA. $x l s x ", " x l s$ $\mathrm{x} "][[3]]$;

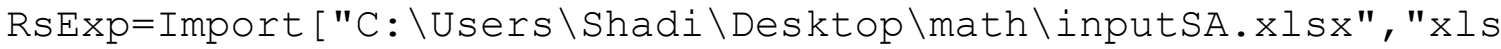
$\mathrm{x} "][[4]]$;

TsExp=Import [ "C: UUsers $\backslash$ Shadi \Desktop \math \inputSA.xlsx", "xls $\mathrm{x} "][[5]]$; 


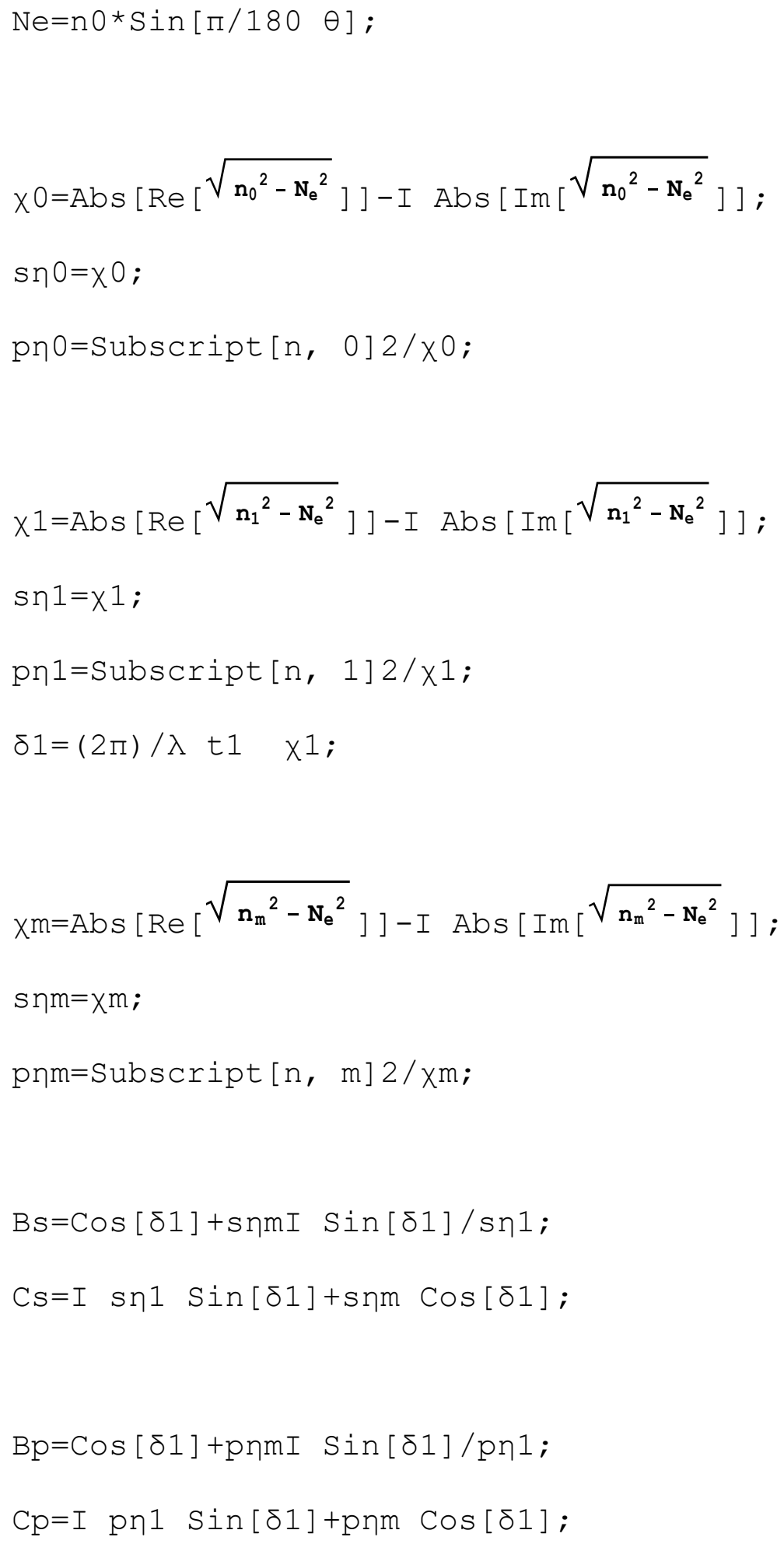




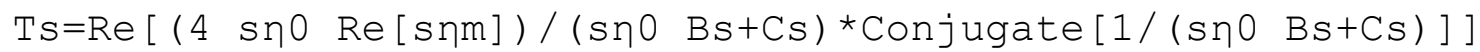

$(4$ sn0 $\operatorname{Re}[\mathrm{snm}]) /(\operatorname{s\eta 0}+\mathrm{snm}) *$ Conjugate $[1 /(\operatorname{s\eta 0}+\mathrm{s \eta m})]$;

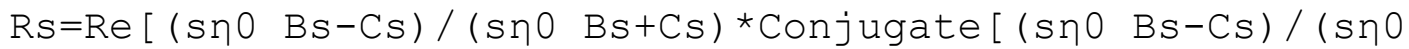

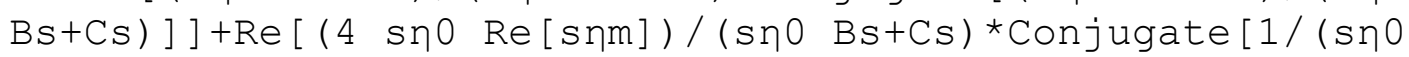

$\mathrm{Bs}+\mathrm{Cs})]] \operatorname{Re}[(\mathrm{s \eta 0}-\mathrm{s \eta m}) /(\mathrm{sn0}+\mathrm{snm}) *$ Conjugate [ (sn0-

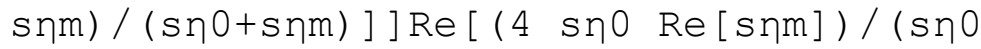

$\mathrm{Bs}+\mathrm{Cs}) *$ Conjugate $[1 /(\mathrm{sn} 0 \mathrm{Bs}+\mathrm{Cs})]]$;

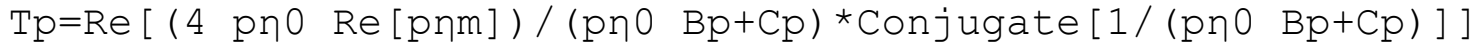

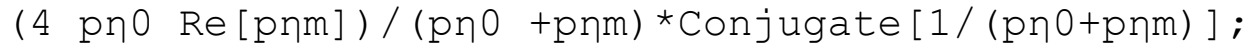

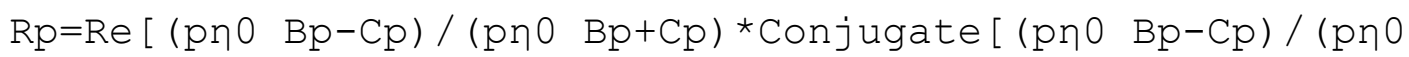

$\mathrm{Bp}+\mathrm{Cp})]]+\operatorname{Re}[(4 \mathrm{p} \eta 0 \mathrm{Re}[\mathrm{p} \eta \mathrm{m}]) /(\mathrm{p} \eta 0 \mathrm{Bp}+\mathrm{Cp}) *$ Conjugate $[1 /(\mathrm{p} \eta 0$

$\mathrm{Bp}+\mathrm{Cp})]] \operatorname{Re}\left[(\mathrm{p} \eta 0-\mathrm{p} \eta \mathrm{m}) /(\mathrm{p} \eta 0+\mathrm{p} \eta \mathrm{m}){ }^{*}\right.$ Conjugate $[(\mathrm{p} \eta 0-$

$\mathrm{p \eta m}) /(\mathrm{p} \eta 0+\mathrm{p} \eta \mathrm{m})]] \operatorname{Re}\left[\left(\begin{array}{lll}4 & \mathrm{p} \eta 0 & \operatorname{Re}[\mathrm{p} \eta \mathrm{m}]) /(\mathrm{p} \eta 0\end{array}\right.\right.$

$\mathrm{Bp}+\mathrm{Cp}) *$ Conjugate $[1 /(\mathrm{p} n 0 \mathrm{Bp}+\mathrm{Cp})]]$;

$\phi=\left(1 /\left(\begin{array}{ll}4 & M\end{array}\right)\right)^{\star} \operatorname{Re}\left[(\operatorname{RpExp}-\mathrm{Rp})^{\wedge} 2+(\mathrm{TpExp}-\mathrm{Tp})^{\wedge} 2+(\operatorname{RsExp}-\right.$

$\left.\mathrm{Rs})^{\wedge} 2+(\mathrm{TsExp}-\mathrm{Ts})^{\wedge} 2\right]$;

$\alpha=\operatorname{Norm}[\phi] ;$

NMinimize $[\alpha,\{\{x, 1.8,2.5\},\{y, 0,0.5\},\{z, 10,20\}\}]$

$\mathrm{x}=2.2728072631741587^{\prime} ;$

$y=-5.443577557124173 ` \star \wedge-9$;

$z=12.214124569559031^{\prime} ;$

ListPlot $[$ Table $[\{\theta[[i]], \operatorname{Rp} \operatorname{Exp}[[i]]\},\{i, 1, M, 1\}]]$;

ListPlot [Table $[\{\theta[[i]], \operatorname{Rp}[[i]]\},\{i, 1, M, 1\}]]$;

ListPlot $[$ Table $[\{\theta[[i]], \operatorname{TpExp}[[i]]\},\{i, 1, \mathrm{M}, 1\}]]$; 


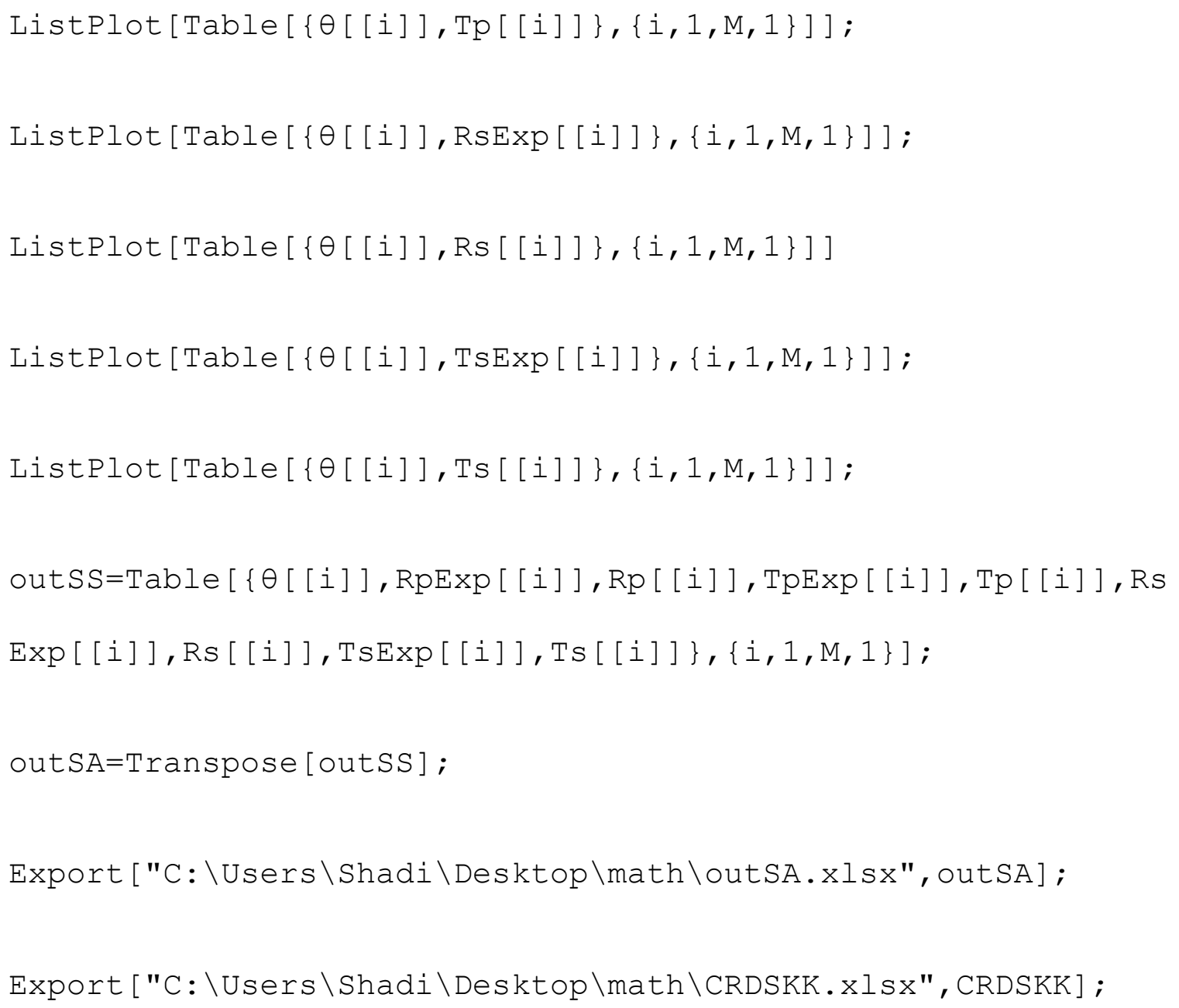


Appendix 8 (b): Mathematica program code used to extract the imaginary part of refractive index. The data of the ring-down times for each sample were converted to single-pass transmittance for p-polarized light using Equation 6-2.This values of transmittance were used with the values of thickness and real part of the refractive part index to estimate the imaginary part of refractive index.

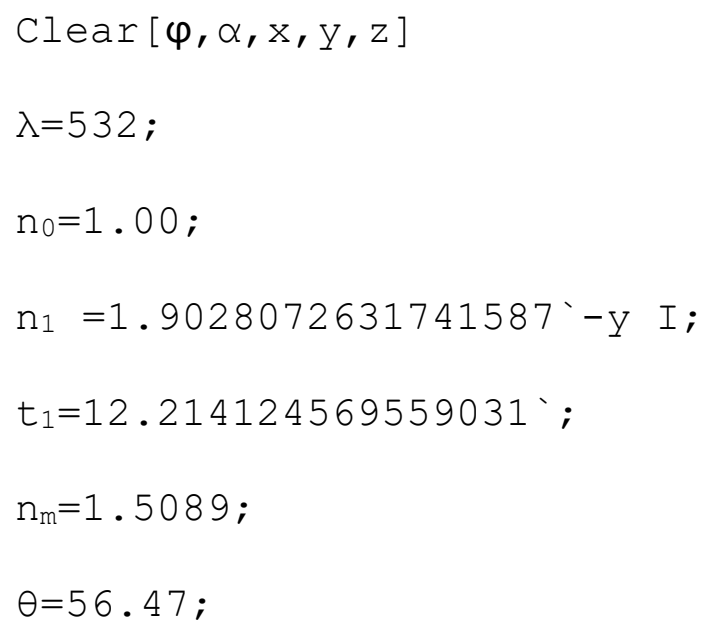




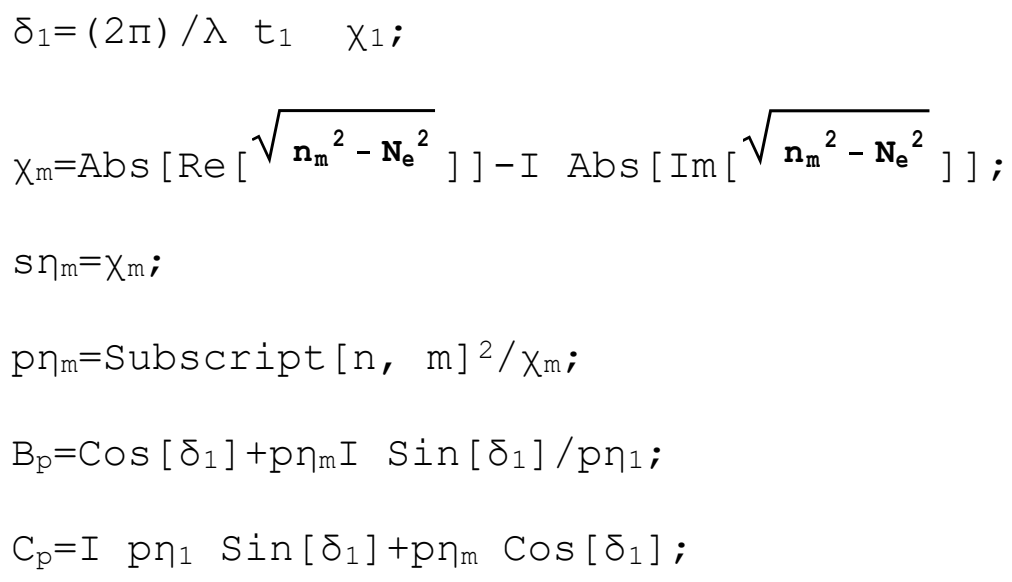

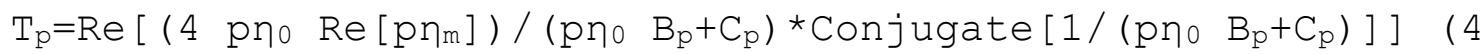
pno $\left.\operatorname{Re}\left[\mathrm{p} \eta_{\mathrm{m}}\right]\right) /\left(\mathrm{p} \eta_{0}+\mathrm{p} \eta_{\mathrm{m}}\right) *$ Conjugate $\left[1 /\left(\mathrm{p} \eta_{0}+\mathrm{p} \eta_{\mathrm{m}}\right)\right]$;

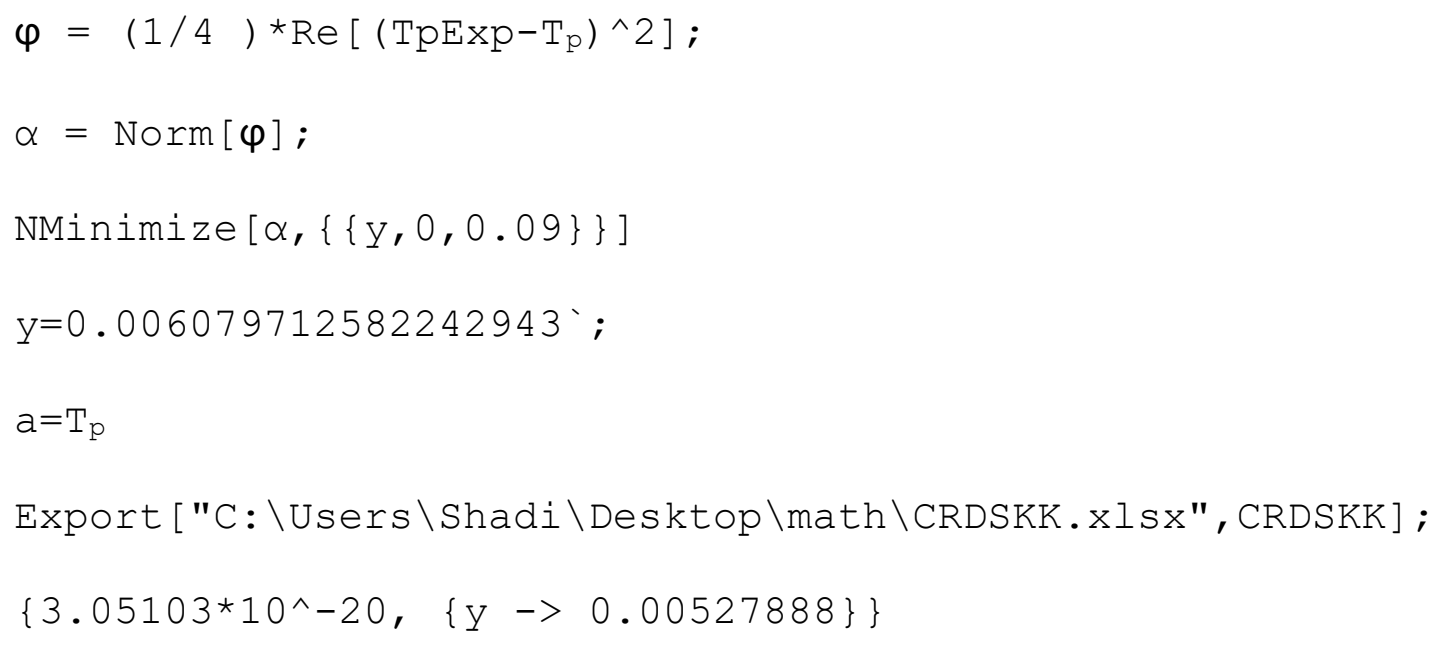


Appendix 9: The results of the single-pass transmittance and ring-down times measurements of ITO ultra-thin films as coated with and without UV exposure at different oxygen flow rates.

Table 1: The single-pass transmittances for p-polarized light at $532 \mathrm{~nm}$ of ITO ultra-thin film were calculated using the data of ring-down times.

\begin{tabular}{|c|c|c|c|c|}
\hline $\begin{array}{c}\text { O } \\
\text { (sccm) }\end{array}$ & T \% (without UV) & T\%(With UV) & $\begin{array}{c}\text { Without UV } \\
\boldsymbol{\tau}(\boldsymbol{\mu} \boldsymbol{s})\end{array}$ & $\begin{array}{c}\text { With UV } \\
\boldsymbol{\tau}(\boldsymbol{\mu} \boldsymbol{s})\end{array}$ \\
\hline 0.6 & 99.6713725 & 99.73164994 & 0.9975 & 1.21783 \\
\hline 0.8 & 99.7016259 & 99.73545388 & 1.09714 & 1.23504 \\
\hline 1 & 99.7020588 & 99.74512835 & 1.09871 & 1.28108 \\
\hline 1.4 & 99.7323297 & 99.76162176 & 1.22087 & 1.36801 \\
\hline 1.8 & 99.7367510 & 99.76742719 & 1.24102 & 1.40148 \\
\hline 2.4 & 99.7578578 & 99.79088025 & 1.34715 & 1.55517 \\
\hline 3 & 99.7737845 & 99.79220336 & 1.44006 & 1.56485 \\
\hline 4 & 99.7777519 & 99.7950196 & 1.46523 & 1.58586 \\
\hline
\end{tabular}

The values of single-pass transmittance for each ITO thin film sample coated with and without UV exposure and at different oxygen flow were recoded in Table 1. The measured cavity ring-down times were used to calculate the single-pass transmittance using Equations 6-2 and 6-3. 
Appendix 10: The results of thickness, real and imaginary parts of the refractive index of ITO ultra-thin films as coated with and without UV exposure at different oxygen flow rates.

Table 1: The calculations results of the thickness, real and imaginary parts of the refractive index of ITO ultra-thin film samples as coated with UV exposure.

\begin{tabular}{|c|c|c|c|}
\hline $\mathbf{O}_{2}$ flow rate(sccm) & $\mathbf{n}$ & $\mathbf{k}$ & $\mathbf{t}(\mathbf{n m})$ \\
\hline 0.6 & 1.85554 & 0.00406194 & 12.8866 \\
\hline 0.8 & 1.8988 & 0.00280415 & 12.9241 \\
\hline 1 & 1.92285 & 0.00235685 & 12.3526 \\
\hline 1.4 & 1.91581 & 0.00200238 & 12.4658 \\
\hline 1.8 & 1.8956 & 0.00193984 & 13.0478 \\
\hline 2.4 & 1.89068 & 0.00172088 & 12.684 \\
\hline 3 & 1.88991 & 0.00158388 & 12.8483 \\
\hline 4 & 1.90016 & 0.00138343 & 12.6403 \\
\hline
\end{tabular}


Table 2: The calculations results of the thickness, real and imaginary parts of the refractive index of ITO ultra-thin film samples as coated without UV exposure.

\begin{tabular}{|c|c|c|c|}
\hline $\begin{array}{c}\mathbf{O}_{2} \text { flow rate } \\
(\mathbf{s c c m})\end{array}$ & $\mathbf{n}$ & $\mathbf{k}$ & $\mathbf{t}(\mathbf{n m})$ \\
\hline 0.6 & 1.86172 & 0.00607971 & 12.3322 \\
\hline 0.8 & 1.89076 & 0.00438389 & 12.4477 \\
\hline 1 & 1.907506 & 0.00421464 & 12.1463 \\
\hline 1.4 & 1.91086 & 0.00319749 & 12.2109 \\
\hline 1.8 & 1.92216 & 0.00277939 & 12.178 \\
\hline 2.4 & 1.91477 & 0.00200008 & 12.6215 \\
\hline 3 & 1.91884 & 0.001806 & 12.2028 \\
\hline 4 & 1.9084 & 0.0016551 & 12.6028 \\
\hline
\end{tabular}




\section{CURRICULUM VITAE}

\section{PERSONAL INFORMATION}

Name: Shadi A. Alnaanah

Email: saalna01@louisville.edu,naana_shadi121@hotmail.com

Phone: 502-822-8891

\section{EDUCATION}

- M.S. of science (physics\&Astronomy)

2016-2018

University of Louisville, Louisville, KY, USA

- M.S. of science (physics)

2007-2010

Mu'tah University, Al-Karak, Jordan

- B.S. of science (physics)

2002-2006

Mu’tah University, Al-Karak, Jordan 


\section{EXPERIENCES}

1- Graduate Teaching Assistant/

2020-present

Department of physics \& Astronomy/ University of Louisville/USA

- Responsible for grading student's lab reports/exams in a timely manner.

- Planned lab sections and course tutorials for students.

2- Research Assistant/ Photonics research Lab/

2016-Present

Department of physics \& Astronomy/ University of Louisville/USA

- Successfully built the Electro-active Evanescent-wave cavity ring-down spectroscopy device.

- Detect and quantify influenza virus molecules by a combination of electrochemical and optical transduction.

- Studies of the adsorption kinetics and the redox properties of a redox species binding to the different molecular interfaces.

- Measure the optical properties of the ITO ultra-thin film during the surface treatment.

\section{3- Lecturer/ Salman Bin Abd-Al-Aziz University}

Saudi Arabia

- Teach several undergraduate courses.

- Assist faculty members with classroom instruction, exams, and courses evaluation. 
- Responsible for checking and assessing students' work grading student's lab reports/exams in a timely manner.

4- Physics \& science teacher/Ministry of education/Jordan

- Physics and science teacher for the middle and secondary schools.

\section{GRANTS AND AWARDS}

- Graduate Network in A\&S (GNAS) grant, University of Louisville, 2018.

- Tafilah Technical University Scholarship, Jordan, 2005.

- Doctoral completion award, University of Louisville, 2020.

\section{RESEARCH INTERESTS}

- Molecular Interfacial Spectroelectrochemical Interaction at the

Nanoscale.

- Immunosensing Using Electro-active Evanescent-Wave Cavity RingDown Spectroscopy (EW-CRDS).

- Continuous Wave CRDS.

- Phase-shift CRDS.

- Thin -Films Properties and Fabrication.

\section{PUBLICATIONS}

1. Shadi A. Alnaanah, Thomas J. Roussel, Jafar H. Ghithan, Aymen H. Qatamin, Mohammed A. Irziqat, Hamzeh Telfah, Jinjun Liu, and Sergio B. Mendes*, 'An 
Electro-Active Interface for Enabling Spectroelectrochemical Investigations in Evanescent-Wave Cavity-Ring-Down Spectroscopy”'. Anal. Chem. 2020, 92, 16, 11288-11296

2. Shadi A. Alnaanah, Melanie K. Dieterlen, Aymen H. Qatamin, Mohammad A. Irziqat, and Segio B. Mendes*, "Effect of the In situ UV treatment on the properties of ultra-thin indium tin oxide film measured by means of the Cavity Ring-Down Spectroscopy ", submitted.

3. Shadi A. Alnaanah, Aymen H. Qatamin, Betty M. Nunn, Martin G. O’Toole, and Sergio B. Mendes*, "Influenza virus immunosensor with electrochemical evanescent-wave cavity ring-down spectroscopy', under preparation.

\section{CONFERENCES AND PRESENTATION}

- Graduate Student Regional Research Conference, '’Evanescent Wave Cavity RingDown Spectroscopy under Potential Modulation for Investigating Interfacial

Processes", poster presentation, University of Louisville, Louisville, KY, 2017.

- Workshop on the materials in Jordan, Amman, Jordan, 2011

\section{MEMBERSHIPS}

- Jordanian physics association.

- Optical society of America. 